JOINT INSTITUTE FOR AERONAUTICS AND ACOUSTICS

lives instor

$1 \mathrm{~N} \cdot 02$

128239

National Aeronautics and

Space Administration

Ames Research Center
JIAA TR - 79

Stanford University

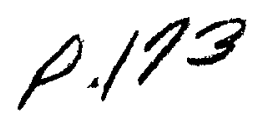

\title{
AN EXPERIMENTAL INVESTIGATION \\ OF STRAIGHT AND \\ CURVED ANNULAR WALL JETS
}

BY

L. C. Rodman, N. J. Wood, and L. Roberts

(IASA-CR-182546) AD EXPERIEEDTAL

IHESSIGATIOA OL SIBAIGHT AMD COBVED ARULAR

NALL JETS (Stanford Oniv-) 173 p CSCL 01A

\author{
N88-18557 \\ Unclas \\ 0128239
}

Stanford University

Department of Aeronautics and Astronautics

Stanford, CA 94305

SEPTEMBER 1987 
JIAA TR - 79

AN EXPERIMENTAL INVESTIGATION OF STRAIGHT AND CURVED ANNULAR WALL JETS .

BY

L. C. Rodman, N. J. Wood, and L. Roberts

Stanford University

Department of Aeronautics and Astronautics Stanford, CA 94305 


\section{ACKNOWLEDGEMENTS}

The authors wish to thank Dr. Rabi Mehta for the generous loan of his LDV system to this work. We are also grateful for the technical assistance provided by Mr. Dale Buermann, Mr. John Mohun, and Mr. Vadim Matte.

Financial support for this work has been provided by the NASA Ames Research Center, under grants NCC 2-173, NCC 2-331, and NCC 2-376. 
Accurate turbulence measurements taken in wall jet flows are difficult to obtain, due to high intensity turbulence and problems in achieving two-dimensionality. The problem is compounded when streamwise curvature of the flow is introduced, since the jet entrainment and turbulence levels are greatly increased over the equivalent planar values. In this experiment, two-dimensional straight and curved incompressible wall jet flows are simulated by having a jet blow axially over a cylinder. In the straight wall case the cylinder has constant transverse radius, and in the curved wall cases the cylinder has a varying transverse radius. Although the wall jets in these cases are annular, adequate "two-dimensional" flow can be obtained as long as the ratio of the jet width to the cylinder radius is small.

The annular wall jet has several advantages over wall jets issuing from finite rectangular slots. Since the slot has no ends, threedimensional effects caused by the finite length of the slot and sidewall interference are eliminated. Also, the transverse curvature of the wall allows close optical access to the surface using a Laser Doppler Velocimetry system.

Hot wire measurements and some Laser Doppler Velocimetry measurements are presented for straight and curved wall jet flows. The results for the straight wall showed good agreement between the annular flow data and rectangular data taken by previous researchers. The one exception was the magnitude of the peak Reynolds shear stress, which was slightly lower for the annular jet than for rectangular jets. For the jets with streamwise curvature, there was agreement between the annular and corresponding rectangular jets for the flow region closest to the slot exit. At far downstream stations, transverse curvature parameters became too large, and the flow began to diverge from the two-dimensional case.

An integral analysis was used as a simple technique to interpret the experimental results. Integral momentum calculations were performed for both straight and curved annular and two-dimensional wall jets. The results of the calculation were used to identify transverse 
curvature parameters and to predict the values of those parameters which would delineate the region where the annular flow can satisfactorily simulate two-dimensional flow. 
Acknowledgements $\ldots \ldots \ldots \ldots \ldots \ldots \ldots \ldots \ldots \ldots \ldots \ldots \ldots \ldots \ldots \ldots$

Abstract.............................

Table of Contents............................ v

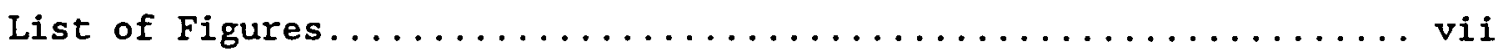

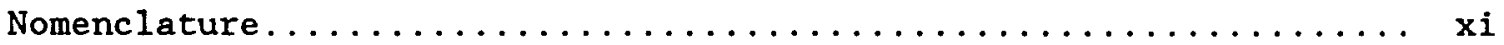

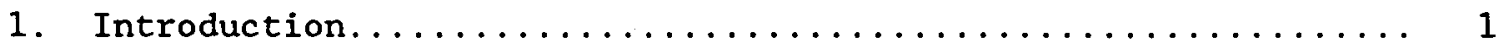

1.1. Wall Jets and Applications................ 1

1.2. Two-Dimensional Wall Jet Characteristics.......... 3

1.3 Summary of Remaining Chapters............... 4

2. Research objectives..................... 7

2.1. Current Prediction Techniques for Curved Wall Jets... 7

2.2. Previous Experimental Difficulties............. 9

2.3. The Annular Wall Jet Concept.............. 12

3. Experimental Description..................... 15

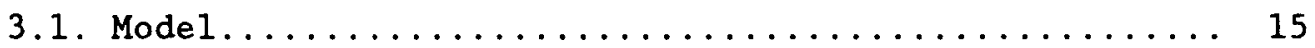

3.2. Equipment and Instrumentation.............. 17

3.2.1. Single Wire Calibration........... 18

3.2.2. Cross-Wire Calibration............ 19

3.2.3. Digital Sampling of the AC Signal....... 21

3.2.4. Data Reduction................ 23

3.2.5. Probe Limitations and Error Analysis...... 24

3.2.6. LDV system................. 26

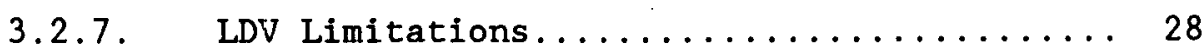

4. Experimental Results......................... 29

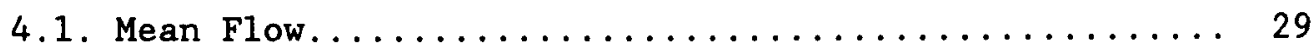

4.2. Turbulence Results.................... 32 


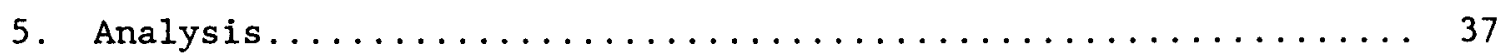

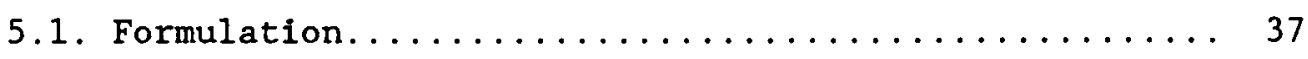

5.2. Self-Similar Mean Velocity Profile............ 38

5.3. Mean Flow Development................... 39

5.4. Shear Stress Calculation................. 44

5.5. Effects of Skin Friction Coefficient........... 46

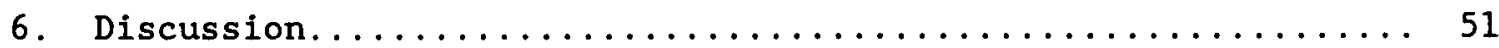

6.1. Evaluation of Experiment................. 51

6.2. Transverse Curvature Effects............... 55

6.3. Streamwise Curvature Effects............... 58

7. Conclusions and Recommendations.................. 63

References................................... 65

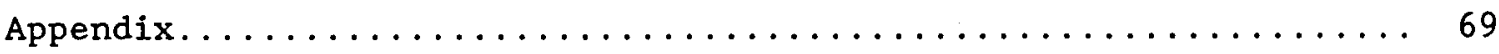

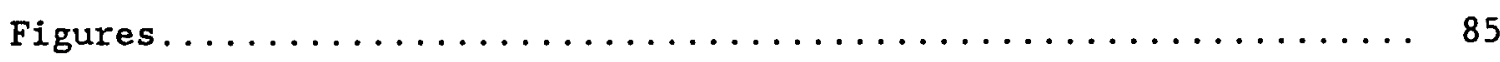




\section{LIST OF FIGURES}

Fig. 1.1 Straight Wall Jet Schematic.

Fig. 2.1 Annular Wall Jet Concept.

Fig. 3.1 Annular Wall Jet Model.

Fig. 3.2 Annular Mode1 Contraction Geometry.

Fig. 3.3 Curved Annular Wall Jet Models.

Fig. 3.4 Support Stand for Annular Wall Jet Models.

Fig. 3.5 Highly Curved Annular Wall Jet Model.

Fig. 3.6 Cross-Wire Probe Orientation.

Fig. 3.7 Flowchart of Cross-Wire Operation.

Fig. 3.8 Effect of Two-Channel Time Lag on Cross-Wire Turbulence Measurements.

Fig. 3.9 Fast Fourier Transform of Streamwise Velocity Component.

Fig. 3.10 Schematic of the Optics Table Layout.

Fig. 3.11 Flowchart of LDV Operation.

Fig. 4.1 Wall Jet Nomenclature.

Fig. 4.2 Spanwise Flow Uniformity in the Straight Annular Wall Jet (Pitot Tube Measurements).

Fig. 4.3 Self-Similar Mean Velocity Profiles in the Straight Annular Wall Jet (Pitot Tube Measurements).

Fig. 4.4 Self-Similar Mean Velocity Profiles in the Straight Annular Wall Jet (LDV Measurements).

Fig. 4.5 Self-Similar Mean Velocity Profiles in the Straight Annular Wall Jet (Cross-Wire Measurements).

Fig. 4.6 Mean Velocity Profiles in the Mildly Curved Annular Wall Jet (Cross-Wire Measurements).

Fig. 4.7 Mean Velocity Profiles in the Highly Curved Annular Wall Jet (Cross-Wire Measurements).

Fig. 4.8 Half-Width Growth in the Straight Annular Wall Jet. 
Fig. 4.9 Maximum Velocity Decay in the Straight Annular Wall Jet.

Fig. 4.10 Half-Width Growth in Curved Annular Wall Jets.

Fig. 4.11 Maximum Velocity Decay in Curved Annular Wall Jets.

Fig. 4.12 Streamwise Normal Stress Profiles in the Straight Annular Wall Jet (Cross-Wire Measurements).

Fig. 4.13 Streamwise Normal Stress Profiles in the Straight Annular Wall Jet (LDV Measurements).

Fig. 4.14 Comparison of Cross-Wire and LDV Measurements in the Straight Annular Wall Jet (Streamwise Normal Stress).

Fig. 4.15 Peak Streamwise Normal Stress Growth in the Straight Annular Wall Jet.

Fig. 4.16 Lateral Normal Stress Profiles in the Straight Annular Wa11 Jet (Cross-Wire Measurements).

Fig. 4.17 Lateral Normal Stress Profiles in the Straight Annular Wall Jet (LDV Measurements).

Fig. 4.18 Comparison of Cross-Wire and LDV Measurements in the Straight Annular Wall Jet (Lateral Normal Stress).

Fig. 4.19 Peak Lateral Normal Stress Growth in the Straight Annular Wall Jet.

Fig. 4.20 Shear Stress Profiles in the Straight Annular Wall Jet (Cross-Wire Measurements).

Fig. 4.21 Shear Stress Profiles in the Straight Annular Wall Jet (LDV Measurements).

Fig. 4.22 Comparison of Cross-Wire and LDV Measurements in the Straight Annular Wall Jet (Shear Stress).

Fig. 4.23 Peak Shear Stress Growth in the Straight Annular Wall Jet.

Fig. 4.24 Streamwise Normal Stress Profiles in the Mildly Curved Annular Wall Jet (Cross-Wire Measurements).

Fig. 4.25 Streamwise Normal Stress Profiles in the Highly Curved Annular Wall Jet (Cross-Wire Measurements).

Fig. 4.26 Lateral Normal Stress Profiles in the Mildly Curved Annular Wall Jet (Cross-Wire Measurements). 
Fig. 4.27 Lateral Normal Stress Profiles in the Highly Curved Annular Wall Jet (Cross-Wire Measurements).

Fig. 4.28 Shear Stress Profiles in the Mildly Curved Annular Wall Jet (Cross-Wire Measurements).

Fig. 4.29 Shear Stress Profiles in the Highly Curved Annular Wall Jet (Cross-Wire Measurements).

Fig. 4.30 Peak Streamwise Normal Stress Growth in Curved Annular Wall Jets.

Fig. 4.31 Peak Lateral Normal Stress Growth in Curved Annular Wall Jets.

Fig. 4.32 Peak Shear Stress Growth in Curved Annular Wall Jets.

Fig. 4.33 Triple Turbulence Component Measurements in the Straight Annular Wall Jet (Cross-Wire Measurements).

Fig. 4.34 Triple Turbulence Component Measurements in the Mildly Curved Annular Wall Jet (Cross-Wire Measurements).

Fig. 4.35 Triple Turbulence Component Measurements in the Highly Curved Annular Wall Jet (Cross-Wire Measurements).

Fig. $5.1 \quad$ Coordinate System for a Body of Revolution.

Fig. 5.2 Assumed Self-Similar Velocity Profile.

Fig. 5.3 Effect of Curvature on Half-Width Growth in Annular Wall Jets.

Fig. 5.4 Effect of Curvature on Half-Width Growth in Two-Dimensional Wall Jets.

Fig. 5.5 Effect of Curvature on Maximum Velocity Decay in Annular Wall Jets.

Fig. 5.6 Effect of Curvature on Maximum Velocity Decay in TwoDimensional Wall Jets.

Fig. 5.7 Computed Shear Stress Profile for the Straight Annular Wall Jet.

Fig. 5.8 Computed Peak Shear Stress Growth in Annular Wall Jets.

Fig. 5.9 Computed Peak Shear Stress Growth in Two-Dimensional Wall Jets.

Fig. 5.10 Effect of Skin Friction Coefficient on Half-Width Growth. 
Fig. 5.11 Effect of Skin Friction Coefficient on Maximum Velocity Decay.

Fig. 5.12 Effect of Skin Friction Coefficient on Shear Stress Profile.

Fig. 5.13 Comparison of Shear Stress Profiles Using Measured and Assumed Mean Velocity Profiles.

Fig. 5.14 Corrected Peak Shear Stress in the Straight Annular Wall Jet.

Fig. 5.15 Corrected Peak Shear Stress in the Mildly Curved Annular Wall Jet.

Fig. 5.16 Corrected Peak Shear Stress in the Highly Curved Annular Wall Jet.

Fig. 5.17 Comparison of Computed and Experimental Shear Stress Profiles in the Straight Annular Wall Jet.

Fig. 6.1 Computed Annular and Two-Dimensional Wall Jet Half-Width Growth.

Fig. 6.2 Computed Annular and Two-Dimensional Maximum Velocity Decay.

Fig. 6.3 Computed Boundaries of Mean Flow Agreement Between Annular and Two-Dimensional Curved Wall Jets.

Fig. 6.4 Comparison of Annular and Two-Dimensional Shear Stress in the Straight Wall Jet.

Fig. 6.5 Comparison of Annular and Two-Dimensional Shear Stress in Mildly Curved Wall Jets.

Fig. 6.6 Comparison of Annular and Two-Dimensional Shear Stress in Highly Curved Wall Jets.

Fig. 6.7 Streamwise Curvature Effects on the Peak Streamwise Normal Stress.

Fig. 6.8 Streamwise Curvature Effects on the Peak Lateral Normal Stress.

Fig. 6.9 Streamwise Curvature Effects on the Peak Shear Stress.

Fig. 6.10 Correlation of Peak Shear Stress with Streamwise Curvature Parameter. 


\section{NOMENCLATURE}

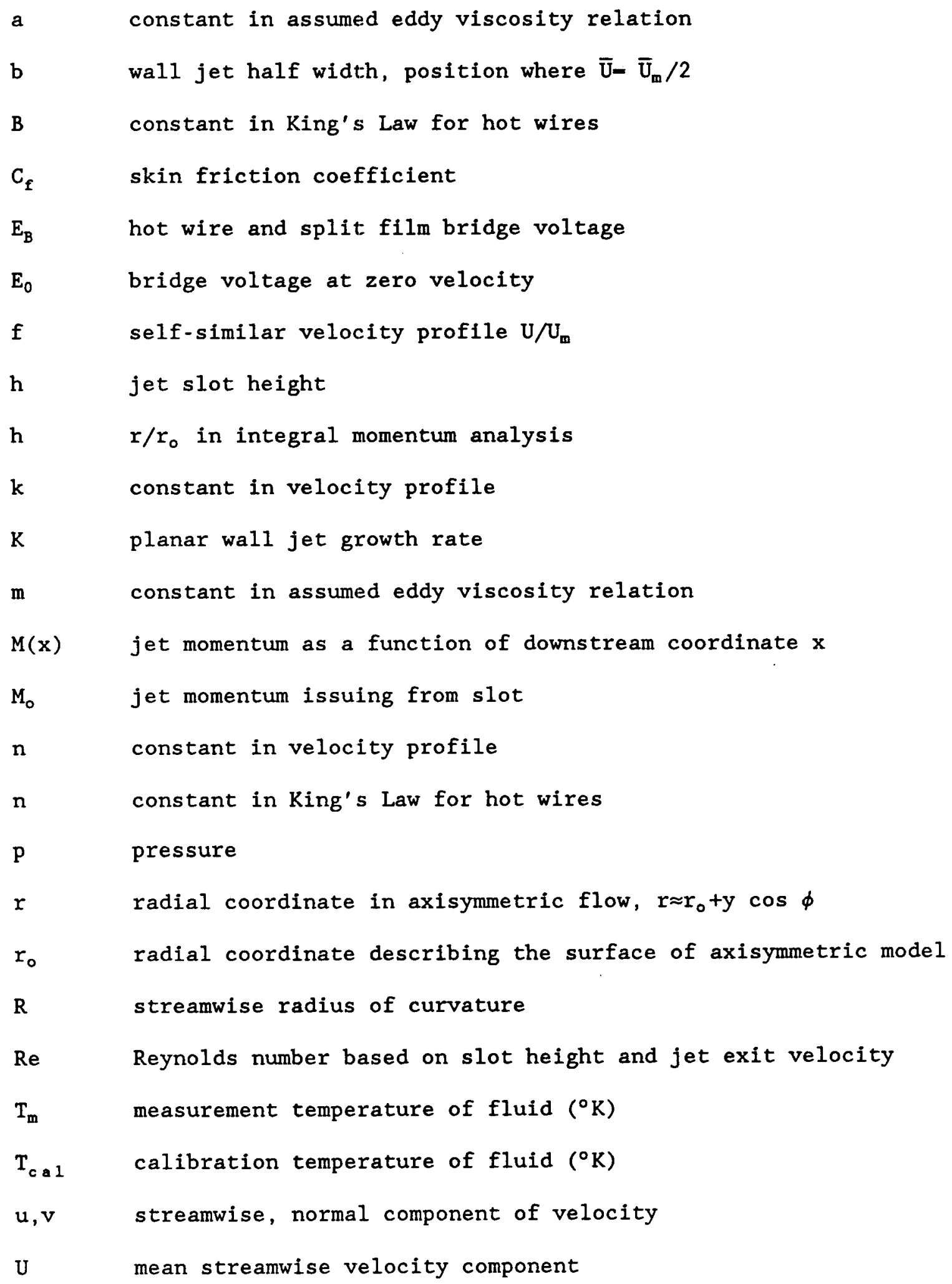




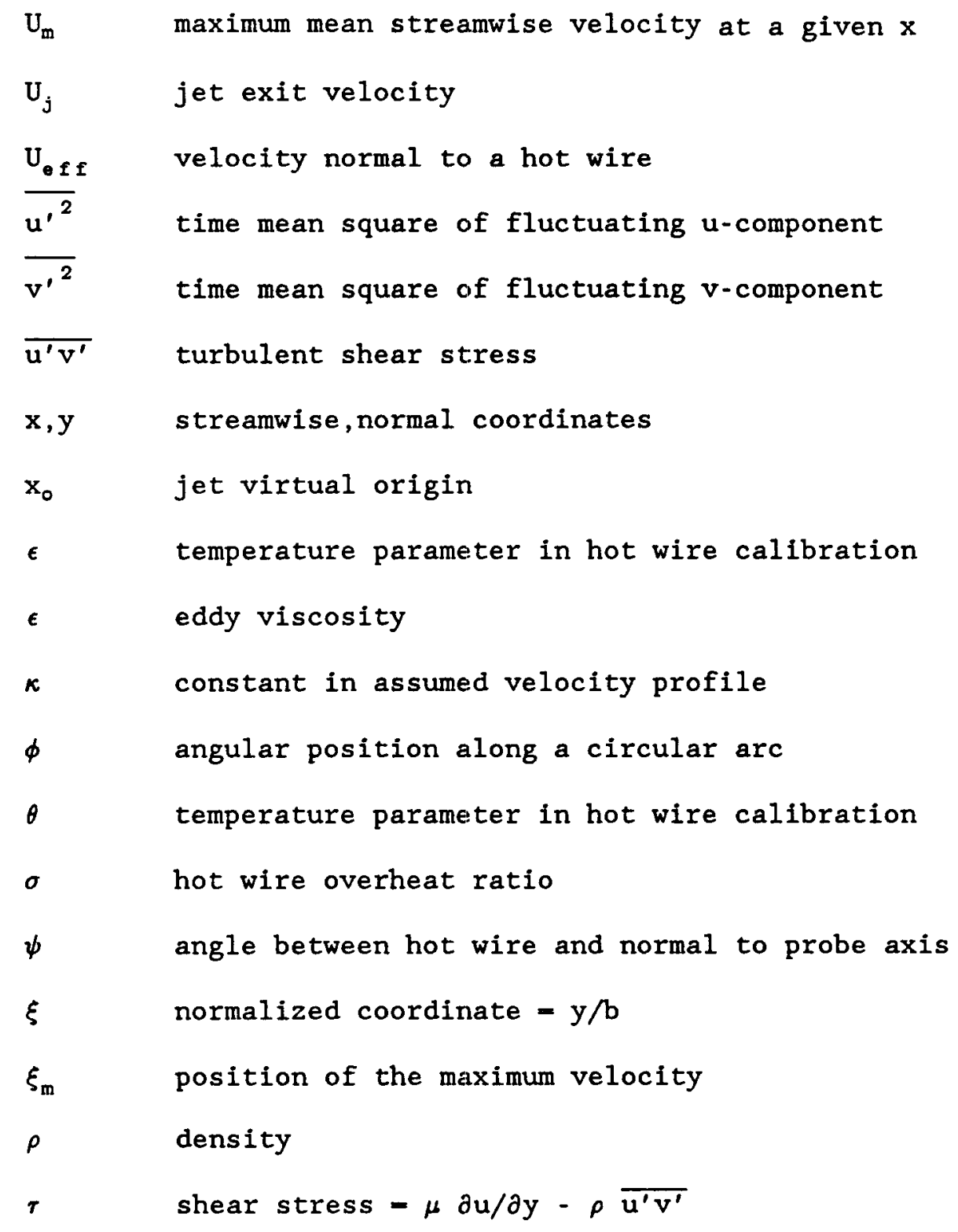


1. INTRODUCTION

1.1. Wall Jets and Applications

The flow of a turbulent jet adjacent to a wall has many practical applications in aeronautics, as well as being of general academic interest. In particular, the strong attachment of a jet to a curved surface is a feature that may be exploited in a variety of flow control schemes, and has been the subject of investigations over the past few decades.

A wall jet is a thin jet of fluid blown tangentially along a wall, where the surrounding fluid may be either at rest or coflowing. The wall jet resembles half of a free jet with a wall boundary layer imposed, and in most practical applications the wall jet will be fully turbulent. Wall jets are thin relative to other dimensions in the flow, and they have a greater streamwise velocity than the surrounding fluid (fig 1.1).

The adjacent wall may be either straight or have streamwise curvature. One of the most interesting and useful features of the wall jet is the Coanda effect, whereby the jet remains strongly attached to a convex surface. As opposed to a curved boundary layer flow, the wall jet can resist the adverse pressure gradient associated with convex curvature long enough to remain attached for turning angles of greater than $200^{\circ} .1$ In addition to strong attachment, curved wall jets display an increase in their mixing with the surrounding fluid compared with straight wall jets. These two properties, wall attachment and increased mixing, enable the wall jet to delay separation of an external stream from a curved surface.

Accordingly, curved wall jets have the most use in aeronautical applications, where blowing is used to delay separation off the upper surface of an airfoil. For applications such as blown flaps, the surface of the airfoil is only mildly curved, so that the jet thickness is about 18 of the streamwise wall radius of curvature. In contrast, circulation control airfoils employ blowing over a highly curved surface, and the ratio of the jet thickness to the radius of curvature 
may become greater than 108 .

Circulation control airfoils employ blowing over a rounded trailing edge to control the lift. Since the trailing edge is not sharp, the usual Kutta condition cannot be enforced and the rear stagnation point is free to move dependent upon the circulation, incidence angle, and free-stream velocity. With blowing, the flow remains attached to the curved surface, delaying separation, and thus allowing the circulation to be controlled as a function of jet blowing. The large increase in circulation for a small movement of the rear stagnation point allows higher lift augmentations for circulation control airfoils than for other blown airfoil configurations, typically by a factor of five.

Circulation control, because of its high lift capabilities at low speeds, has many applications in the design of helicopters and V/STOL aircraft. Blowing can be used to control the lift on a helicopter rotor and thus replace mechanical collective and cyclic pitch control. Leading edge blowing, where a jet of air is blown tangentially over the leading edge, may be used to increase the lift on a helicopter rotor blade when it is operating in the reversed flow region on the retreating blade side at high advance ratios. The blowing is in the opposite direction of the free stream during most of the rotor cycle, but in the reversed flow region it acts as trailing edge blowing and produces positive lift compared to the usual retreating blade stall. This reduction of retreating blade stall and corresponding improvement in rotor disc efficiency would enable helicopters to fly at higher advance ratios. For V/STOL applications, circulation control is capable of providing a transition between low-speed helicopter flight and highspeed fixed wing aircraft flight, such as with the $X$-wing stopped rotor vehicle. 2

Straight wall jets have additional applications in film cooling, since heat transfer from a wall may be increased by the use of surface blowing. ${ }^{3}$ However, the primary interest in this study is in the aerodynamic properties of wall jets, and in particular the effects of streamwise curvature on the turbulent structure. 


\subsection{Two-Dimensional Wall Jet Characteristics}

The typical wall jet velocity profile appears to be half of a free jet with the addition of a thin boundary layer next to the wall. However, this simplification is not completely accurate, since the presence of the wall is felt throughout the entire flow field, and the outer portions of the flow strongly affect the inner regions. The boundary of the inner and outer regions of the flow is considered to be at the position of maximum velocity in the jet, where the velocity gradient is zero (fig. 1.1). The wall damps the size of the large eddies in the outer region, causing decreased entrainment for a wall jet than for a free jet. This results in a slower growth rate of the jet width and a slower decay rate of the maximum velocity compared with a free jet. Pressure reflections from the wall impede the transfer of turbulent energy from the streamwise to normal direction, hence the Reynolds stress normal to the wall $\left(\overline{v^{\prime}}\right)$ is lower in a wall jet flow than in a free jet. 4

The outer region also affects the inner region of the flow. Positive turbulent shear stress from the outer region encroaches into the inner layer, where the turbulent shear stress is negative, thereby displacing the point of zero shear stress closer to the wall. The position of zero shear stress thus does not coincide with the position of zero mean velocity gradient, as is assumed in simple eddy viscosity turbulence models. A logarithmic inner-layer velocity profile typical of boundary layers exists in a wall jet, but only over a small portion of the near-wall flow. This region eventually decreases downstream in a wall jet, since the relative strength of the outer region to the inner region increases as the jet develops. The complex interaction between the outer shear layer and the inner wall layer in a wall jet flow provides a challenge to investigators to predict these effects.

Straight wall jets issuing into still air have been shown to be self-similar, so that non-dimensionalized flow properties are independent of downstream location. 5 The similarity is not exact, since the inner and outer regions develop at slightly different rates; however, it is an extremely good approximation. If the wall is curved, however, 
similarity should only occur if the ratio of the streamwise coordinate to the radius of curvature remains constant, such as with a logarithmic spiral. 6 With this type of surface, the ratio of the jet width to the radius of curvature remains constant, even though the jet is growing. For other curved walls such as circular arcs, the jet will not be self-similar, since as the jet grows the ratio of the jet width to radius of curvature increases. Straight wall jets with an external stream have been shown to be self-similar as long as the ratio of the external velocity to the jet maximum velocity remains constant, such as in an adverse pressure gradient. 7

Streamwise curvature produces an extra strain rate in the turbulent flow, in addition to the simple strain found in straight wall jets. The effects of curvature on the turbulent structure, and thus the mean flow, have been documented experimentally and have been found to be unexpectedly large. In attempts at predicting these flows, it has been found that writing the time-averaged Navier-stokes equations in curvilinear coordinates and using a simple turbulence model will underpredict the effects of curvature by an order of magnitude. 8 Thus, the prediction of curvature effects on wall jet flows remains a challenge to researchers, as more complicated flow modeling techniques are required.

\subsection{Summary of Remaining Chapters}

The physics of the flowfield in highly curved wall jets is not fully understood. Experiments are necessary for the study of curvature effects on the turbulent structure in these flows, and to aid in developing calculation schemes. Chapter 2 will examine some of the prediction schemes used to calculate curved wall jet flows, as well as reviewing previous experimental research in this area. In light of some of the difficulties faced by previous researchers, the present experimental objectives will be defined, and a research plan developed.

Chapter 3 will describe in detail the experimental apparatus and instrumentation used in the present investigation. A complete documentation of the experimental results is contained in Chapter 4 . In 
Chapter 5, an analysis is developed which is used to interpret the experimental results. The discussion of results is contained in Chapter 6, and finally, the conclusions and recommendations are in Chapter 7 . 


\section{RESEARCH OBJECTIVES}

It is necessary to obtain detailed turbulence measurements in highly curved wall jets in order to develop prediction techniques for these flows. The objectives for this experiment were based on this need for an experimental investigation of incompressible curved wall jets.

\subsection{Current Prediction Techniques for Curved Wall Jets}

At present, the ability of turbulence models to predict the effects of streamwise curvature on wall jets is limited. Although the use of empiricism may be used in the calculation of mild curvature effects, the profound effects of high curvature on the turbulent structure have so far been elusive to existing turbulence models.

The simplest turbulence models employ some type of eddy viscosity approximation, relating the turbulent shear stress to the mean rate of strain $(\partial u / \partial y)$. Either a mixing length approximation ${ }^{9}$ or the transport equations for the turbulent kinetic energy and length scale (twoequation models) are used for the eddy viscosity. The eddy viscosity approximation is not valid even with a straight wall jet, since, as was mentioned previously, the point of zero shear stress does not correspond to the point of zero mean velocity gradient. However, this difference is small enough to neglect in most practical applications, and the eddy viscosity models do work in many flow situations, even though their physical basis is suspect.

Mixing length models have been used with some success in straight wall jets, as long as the mixing length varies in such a way as to account for the wall damping on the normal velocity fluctuations. 10 However, this method is limited by a lack of universality.

Streamwise curvature introduces an additional rate of strain $(\partial v / \partial x)$ to the flow, suggesting that for a mixing length model, the value of the existing shear stress should be increased by a factor

$$
1+(\partial v / \partial x) /(\partial u / \partial y)
$$


Vast experimental evidence shows that the actual effect of curvature is to increase the stress by a factor

$$
1+10(\partial v / \partial x) /(\partial u / \partial y)
$$

for mildly curved flows. This empirical constant is not universal, and for highly curved flows, there is no similar empirical correction. The magnitude of the changes due to curvature suggests that there is a large effect on the processes by which the Reynolds stresses are generated and maintained which are not accounted for in the mixing length model when the equations of motion are written in curvilinear coordinates. $^{8}$ For these reasons, mixing length models have been found to be inadequate for curved flows, due to their inability to account for the secondary strain due to curvature.

Eddy viscosity models using transport equations also cannot predict curvature effects if the equations are written in curvilinear coordinates. Even in the straight wall jet, two-equation models will overpredict the spreading rate of the jet unless specific corrections to account for wall damping are included. Improved corrections in curved flows, such as modifying the coefficient of the length scale, bring better agreement with experiment, yet are still not general enough for different curved flow cases. 11

Models which use the Reynolds stress transport equations for closure are the most promising for accuracy, but at the cost of introducing computational complexity. These schemes involve models for each unknown in the transport equation. Several proposals for these models have been reviewed by Launder \& Rodi.11 Reynolds stress transport models have several advantages over eddy viscosity models. The most important is that it is possible to model the pressure-reflection term in the transport equations, which accounts for the wall damping of the turbulence intensity normal to the wall. It is also possible to exactly calculate the generation term for the shear stress from the interaction between the Reynolds stresses and the velocity gradients. These models can also accurately predict a nonzero shear stress at the velocity maximum. 
The Reynolds stress transport models written in curvilinear coordinates with no empirical input successfully predict the effects of mild curvature on wall jets. However, agreement deteriorates for highly curved jets, and the growth rates of these jets tend to be underpredicted.

A promising compromise between the successful Reynolds stress transport models and the simpler eddy viscosity models is the algebraic stress model. In these models, the turbulent processes are approximated in terms of the turbulent kinetic energy. Only one transport equation for the kinetic energy is needed, and the turbulent stresses are computed algebraically. ${ }^{11}$ These models are successful in predicting mildly curved wall jets, but there is less agreement with experiment for high curvature. A drawback to these models is that they do not accurately reflect the high influence of the shear stress from the jet region on the wall region. Also, like the eddy viscosity models, they cannot predict non-zero shear stress at the velocity maximum. Rodi \& Scheuerer ${ }^{12}$ compare the performance of an algebraic stress model with two-equation eddy viscosity models.

The future development of computational schemes will rely on the availability of measurements taken in highly curved wall jet flows. Thus, the primary objective of the present work was to take detailed and accurate measurements of the Reynolds stresses in highly curved wall jet flows.

\subsection{Previous Experimental Difficulties}

As higher order computational schemes are being developed, it is essential to obtain accurate turbulence measurements in highly curved flows. Previous research on curved wall jets have been either on logarithmic spirals or on circular arcs. Although self-similarity is a useful feature when comparing experimental data with computational schemes, the spiral is not a practical shape from an engineering viewpoint. The ability to predict the wall jet flow about an arbitrary shape is the eventual goal of researchers, and turbulence models should be able to predict both similar and non-similar curved flows. 
Measurements have been difficult to obtain in wall jet flows, due to high intensity turbulence and the complexity of achieving satisfactory two-dimensional flow. In a review of experimental wall jet research, Launder \& Rodi ${ }^{4}$ discovered that the majority of wall jet data is contaminated by poor two-dimensionality, as assessed by a momentum conservation technique. Various researchers at McGill University investigated the causes of three-dimensional effects. Feketel found that irregularities in the wall leading to the slot 1 ip and the edge of the lip itself caused major lateral variations in the jet thickness. If the slot lip is rounded, the Coanda effect causes the issuing jet to curl around the lip, creating a locally thicker flow. Since the surface pressure is lower in these regions than in an adjacent thin jet region, a local inflow is created. Guitton ${ }^{13}$ discovered that a squared-off slot lip allows a more uniform flow than a knife edge.

Another major cause of three-dimensional effects is the creation of vorticity at the ends of a finite slot. Endplates are typically used along the sides of a wall jet to reduce transverse spreading and improve two-dimensionality. However, Guitton ${ }^{13}$ and Gartshore \& Hawaleska 14 found that the interaction between the sidewall boundary layer and the jet causes a secondary flow which can affect the wall jet over its entire width. They discovered that close to the endplates, the flow along the surface moves inward towards the jet centerline, but the flow away from the wall, near the velocity maximum, tends outward towards the sidewalls. This creates a U-shaped secondary flow which splits at the endplates: the flow above the velocity maximum adds to a vortex, and the flow closer to the surface acts to expand the boundary flow.

Guitton's solution was to use a "ladder" arrangement of endplates. Three separate endplates were placed along the jet flow, each displaced relative to the previous one so as to bleed off the sidewall boundary layer. In this way, the boundary layer was kept thin enough to greatly reduce secondary flows. Other researchers ${ }^{15}$ have used tangential blowing on the endplate near the jet slot to energize the wall boundary layer and prevent separation. 
A third cause of flow nonuniformity is the jet contraction. The presence of concave curvature in the contraction has been implicated as a cause of longitudinal vorticity, which causes spanwise variations in the flow. 16 In jet flows, there are often set screws placed periodically inside the slot to adjust the slot height along the span. Vorticity is created at these screw positions, which will propagate downstream. For very large span models, such supports inside the contraction are necessary to maintain the slot geometry under high pressure.

For any configuration, to reduce three-dimensional effects, the jet slot should have a very large aspect ratio in order to remove the sidewall vorticity as far from the center span of the model as possible. However, most practical experiments would be constrained by physical size or volume flow limitations. Also, as the jet span is increased, it becomes more difficult to accurately machine the slot and to maintain its geometry.

These difficulties may be controlled in a straight wall jet model if sufficient care is taken when designing the experiment. However, curved wall jets are characterized by enhanced mixing of the jet flow and the surrounding fluid, and there is faster spanwise transport of disturbances in these jets. This amplifies all of the above threedimensional effects in the flow, and it would be extremely difficult to design an experiment which was not contaminated by three-dimensionality in some way.

Previous researchers have made measurements on wall jets over circular arcs, yet none have been successful in achieving good flow uniformity over a highly curved wall. Newman ${ }^{5}$ and then Fekete ${ }^{1}$ at McGill University were the first to attempt to measure such a flow, but at that early stage, only limited turbulence measurements of the streamwise normal stress were made. Wilson 17,18 undertook a detailed study of a highly curved wall jet and measured all three Reynolds stresses, but reviewers ${ }^{4}$ have cited his experiment as being affected by three-dimensionality.

Alcaraz 19,20 has presented detailed measurements in a mildly curved wall jet, including the Reynolds stresses and various triple 
components. To achieve flow uniformity, he chose a very low slot height: radius of curvature ratio. His slot was chosen to be large to avoid unsatisfactorily low Reynolds numbers. Since his model was only mildly curved, his experiment did not address the need for high curvature data. A recent experiment by Kobayashi \& Fujisawa 21 evaluated the Reynolds stress and skin friction of wall jets over several circular surfaces, yet the presence of a straight wall section prior to the start of the curvature makes their data difficult to correlate with other experiments.

In light of the difficulties experienced by previous researchers in achieving adequate flow uniformity, a second objective of the present investigation was to devise and validate an experimental approach which would avoid many of the problems associated with three-dimensional effects.

\subsection{The Annular Wall Jet Concept}

The objective of this work is to obtain a set of Reynolds stress measurements in straight and curved incompressible wall jets in still air, and to achieve a flow quality as close to two-dimensional as possible. There are two options available to achieve the second objective. The first option is to use a rectangular slot model with a very large span, similar to Alcaraz. 19 The advantage to this type of model is that it removes the source of three-dimensional effects far from the center span, where measurements are made. However, increased curvature causes an increase in the transverse rate of disturbance, so that the span would have to be corresponding larger for highly curved flows. Thus, the disadvantages to a large span model are its cumbersome size in a laboratory, and the volume flow limitations of the available air supply. Other disadvantages are the difficulties in accurately machining a large slot and maintaining its geometry under high internal pressure.

An alternative approach is to use an annular model. With an annular model, the jet is blown axially along the outside of a cylinder (fig. 2.1). Since the slot is wrapped completely around the cylinder, there 
are no ends to the flow and end effects are eliminated. Other advantages to annular models are that rounded slots are more easily machined than rectangular slots, and the slot dimensions may be made to closer tolerances. A central support structure within the cylinder can be used to control the slot height uniformity, thus eliminating the need for a support structure within the contraction. With these advantages, the annular model should produce a high quality "two-dimensional" flow. Also, an annular model would allow improved optical access for a 2-component Laser Doppler Velocimetry (LDV) system. When measuring the normal velocity component with an LDV system in a planar experiment, keeping the laser beams oriented such that the beam axis is parallel to the wall causes wall interference with the beam to be unavoidable near the surface. However, with the wall curving transversely away from the beams, as is the case for the wall cylinder, it is possible to make measurements much closer to the surface before beam interference occurs.

The annular flow model was chosen for this experiment. The ratio of the jet slot height to cylinder radius was small $(0.68)$, so that transverse curvature effects were expected to be negligible. Cylindrical models have been used in earlier experiments. In 1965, McGahan ${ }^{16}$ performed a wall jet experiment over a cylinder in a wind tunnel. He obtained good results, although turbulence measurements were not made. More recently, Bachalo \& Johnson 22 developed a cylinder for boundary layer studies. Both increased spanwise flow uniformity and laser access were cited as reasons for using a cylindrical rather than a planar boundary layer flow.

A major disadvantage to annular models is the introduction of transverse curvature to the flow, which is not present in the twodimensional case. For a curved wall jet, the annular model causes added complexity since the cylinder radius decreases with streamwise distance. Therefore, a major emphasis of this work was the prediction of transverse curvature effects, so that any departure from twodimensionality could be identified and minimized.

To accomplish this, a simple momentum integral technique was used to compute the flow over both annular and two-dimensional straight and 
curved wall jets. A comparison of the results led to a criterion for regions where the annular flow is a satisfactory approximation to two-dimensional flow. 
3. EXPERIMENTAL DESCRIPTION

\subsection{Mode1}

An annular model was chosen for this experiment in order to achieve a high quality wall jet flow with minimal three-dimensional effects. It was anticipated that the elimination of the slot ends, as well as carefully controlled machining, would allow for a uniform flow along the span. The annular wall jet model was designed so that transverse curvature effects were minimized. The cylinder radius was to be as large as possible compared to the jet width, yet was also to have sufficient curvature to allow good laser access to the surface, as a function of the beam angles.

The model consisted of two cylinders (fig. 3.1). The larger, outer cylinder housed the plenum chamber and formed the slot lip of the nozzle. Air from the blower was input to the model through a central support tube. The volume of the chamber was large enough for the flow to settle and to remove circumferential nonuniformities. The slightly smaller, inner cylinder formed the wall for the jet, and was also independently supported by the central tube. This eliminated any need for a support structure within the contraction, thus allowing a clean exit flow. The contraction was designed so that there was a minimum of concave curvature. The presence of concave curvature within a nozzle contraction may result in longitudinal vortices within the wall boundary layer, which have been implicated as the cause of spanwise nonuniformities in wall jet flows. 16 Figure 3.2 shows the contraction geometry in detail.

The model cylinder was chosen to have a radius of $20 \mathrm{~cm}$, the limit of the available machining capability. This radius allowed laser access to within $0.4 \mathrm{~mm}$ from the wall, with the beam array aligned tangentially to the surface. The slot height was $1.3 \mathrm{~mm}$, and the slot lip was cut square, with a thickness of $0.25 \mathrm{~mm}$. The straight wall extended $23 \mathrm{~cm}$ downstream. With a jet exit velocity of $100 \mathrm{~m} / \mathrm{s}$, the Reynolds number based on slot height was 9,000. A similar annular wall jet model of smaller radius $(5 \mathrm{~cm}$ ) but identical slot height had also 
been built. It was designed to fit in a wind tunnel for the study of wall jets with an external stream. Without the external stream it could be used as a comparison with the larger model to isolate the effects of transverse curvature, since the jet width:cylinder radius ratio differed by a factor of 4 between the two models.

The annular model concept was extended to walls with streamwise curvature. Two models were built, one with mild streamwise curvature and one with high curvature (fig. 3.3). For engineering practicality, the surfaces were chosen to be circular arcs. These annular flows could then be compared to previous experiments on rectangular jets over circular arcs.1,5,17,19,21 In particular, the work of Alcaraz ${ }^{19}$ has been cited by reviewers as being a thorough study of the turbulence characteristics of a mildly curved wall jet, and the flow quality of this work was determined to be very high. The parameter which signifies the strength of the streamwise curvature is $h / R$, the ratio of the jet slot height to streamwise radius of curvature. The ratio $h / R$ is zero for a straight wall jet and increases as the jet becomes more highly curved. The curvature of the mildly curved annular model was chosen to match that studied by Alcaraz (h/R-0.0032), to enable a direct comparison of the results.

The highly curved surface was chosen to minimize transverse curvature effects. A streamwise radius of $5 \mathrm{~cm}$, with the wall covering $90^{\circ}$ of arc, produced an $h / R=0.0245$ and extended 50 slot heights downstream. The mildly curved model had a streamwise radius of curvature of 41.8 $\mathrm{cm}$. The curved wall extended $25 \mathrm{~cm}$ downstream, covering $35^{\circ}$ of arc and approximately 190 slot heights. These two models were designed to interchange with the straight cylinder model, such that the same slot lip and contraction were used for all three configurations. The slot height varied slightly between the models, due to slight differences in the annular radius of each surface.

All three models were supported by a steel framework which supported the model in a vertical orientation (fig. 3.4). The small amount of play between the central support tube of the model and the bushings on the upper cylinders allowed the slot height to be finely adjusted. The adjustment was made with three screws which extended from the top of 
the support structure and pushed down on the top of the model. These screws could be adjusted until the slot height was uniform about the model circumference. No internal supports were required within the contraction itself.

The highly curved model had a flat surface faired with the curvature on the top of the model, and a vertical tube protruded out of the center, which allowed the jet to flow upwards after exiting the curved section (fig. 3.5). On the top of this tube a spoked plate was used to support the model and to adjust the slot height.

\subsection{Equipment and Instrumentation}

Mean flow measurements in the wall jet were taken using a pitot pressure tube, and turbulence measurements were made with single and cross hot-wires and a two-component Laser Doppler Velocimetry (LDV) system. The mean velocity profiles and three Reynolds stresses were measured.

The wall jet air was supplied by a 7 1/2 HP centrifugal blower, which provided $0.21 \mathrm{~m}^{3} / \mathrm{s}$ at $14,000 \mathrm{~N} / \mathrm{m}^{2}$ gauge. Losses through the connecting hose and the model decreased the jet exit gauge pressure to about 6000 $\mathrm{N} / \mathrm{m}^{2}$, giving an exit velocity around $100 \mathrm{~m} / \mathrm{s}$.

The blower heated the air to approximately $20^{\circ} \mathrm{C}$ above room temperature. This caused significant temperature gradients in the jet, both across the jet profile and along the streamwise direction. The hot jet would not affect the pitot or the LDV measurements, but would affect the hot-wire anemometry results. A heat exchanger was used between the blower and the model to cool the air supply. The heat exchanger was designed to minimize the pressure losses through it so that the jet Reynolds number could be maintained. The hot air passed though a large cylinder containing a copper coil. The cylinder was $84 \mathrm{~cm}$ long, with a $13 \mathrm{~cm}$ diameter. The copper coil was a 15 meter long tube, $6.4 \mathrm{~mm}$ in diameter, coiled at $13 \mathrm{~mm}$ spacing. Chilled water flowed through the coil, thus cooling the air. This arrangement cooled the plenum temperature by $8^{\circ}-10^{\circ} \mathrm{C}$. Typical temperatures in the wall jet flow 
ranged from $0^{\circ}$ to $7^{\circ} \mathrm{C}$ above room temperature. A temperature correction was employed for the hot wire measurements (Sec. 3.2.1.).

Turbulence measurements were made using two techniques. Laser Doppler Velocimetry measurements were used to take measurements of high turbulence intensities without probe interference. However, as an LDV system was available for this experiment on a limited basis, only measurements of the straight wall jet were made. The LDV results were used to demonstrate the feasibility of taking measurements close to a transversely-curved wall. The majority of the turbulence measurements were made with cross-wires, and these results were compared with the limited LDV results.

\subsubsection{Single Wire Calibration}

A single hot wire probe was initially used to measure the longitudinal streamwise turbulence intensity $\mathrm{u}^{{ }^{2}}$. The wire was calibrated using standard calibration equipment (DISA type 55D90), and was calibrated for the velocity range 4-100 m/s. A high pressure air supply (separate from the centrifugal blower) was used for calibration, and this air was at room temperature $\left(20^{\circ} \mathrm{C}\right)$.

The wire was calibrated both with and without a linearizer. The nonlinear calibration used a King's Law curve fit to relate bridge voltage to velocity:

$$
E_{B}^{2}-E_{0}^{2}+B U^{n}
$$

The two calibration methods yielded the same velocity results when comparing the mean velocities. It was decided to take measurements using the nonlinear calibration, since the temperature correction applied was simplest for the nonlinear case, and because there was no difficulty in implementing the King's law equation in software. These measurements were taken prior to the construction of the heat exchanger, so the jet temperatures were high.

A temperature correction 23 was used for all of the hot wire measurements. The calibration equation (3.1) was found at room temperature 
$T_{c a 1}$, and the measurements were taken at a higher temperature $T_{m}$. Setting

$$
\text { and } \quad \begin{aligned}
\epsilon & =\left(\mathrm{T}_{\mathrm{cal} 1}-\mathrm{T}_{\mathrm{m}}\right) / \mathrm{T}_{\mathrm{cal}} \\
\theta & =\mathrm{T}_{\mathrm{wr} \bullet} / \mathrm{T}_{\mathrm{cal}} \\
\sigma & =\theta-1,
\end{aligned}
$$

all the measured mean voltages were corrected by the factor

$$
E_{B, \operatorname{corr}}=E_{B, m}(1-\epsilon / 2 \sigma) \text {. }
$$

The normalized turbulence intensity was found from the measured voltage fluctuation $e_{R M S}$ by the relation

$$
\sqrt{u^{\prime}{ }^{2}} / U=2 n\left(E_{B, m} e_{R M S}\right) /\left(E_{B, m}{ }^{2}-E_{0, m}{ }^{2}(1+\epsilon / \sigma)\right)
$$

These voltage fluctuations were measured using an analog RMS meter. The temperature correction is valid for small changes in fluid temperature relative to the difference between the wire temperature and the fluid temperature, $1 . e$. If $\epsilon / \sigma$ is small. In this case,

$$
\epsilon / \sigma=-.092 \text { @ } \mathrm{T}_{\mathrm{m}}=40^{\circ} \mathrm{C}
$$

and $\epsilon / \sigma=-.028$ @ $\mathrm{T}_{\mathrm{m}}=25^{\circ} \mathrm{C}$.

Since there were large temperature gradients present in the jet, temperature profiles were measured with a thermocouple at various downstream locations. Local temperatures could then be input into the correction formula.

\subsubsection{Cross-Wire Calibration}

Cross-wire probes were also calibrated with DISA type 55D90 calibration equipment. The DISA system was equipped such that the probe could be inclined at various known angles to the calibration jet flow. The angle of each wire relative to the probe axis was determined by finding the angle where the bride voltage output was a maximum for a given calibration velocity. Once the orientation of the wires in the 
probe was known, a calibration curve was found for each wire as it was aligned normal to the flow, as if it were a single hot wire. The curve fit determined the constants $B$ and $n$ in eq. (3.1). The constant $E_{0}$ (the zero flow voltage) was measured by aligning the wire vertically in still air. The measured $E_{\circ}$ was multiplied by the correction factor .92, and the corrected value was squared and used in the calibration equation. 24

At each measurement point in the flow, the voltage output from each of the constant temperature bridges (DISA 55M10) was sampled with a long time average (2000 samples over ten seconds) to obtain the mean voltages. To maximize the resolution of the $A / D$ converter, the $D C$ component of the bridge output was removed and the remaining fluctuating component was amplified (NEF Differential Instrumentation Amplifier Model 126): The A/D converter (Data Translation DT2821) in the IBM AT personal computer sampled signals between \pm 10 volts, and converted those voltages to an integer between 0 and 4095. The gain on the AC signal was set to maintain the voltage within the \pm 10 volt range. The mean bridge voltage was then added to each sampled $\mathrm{AC}$ voltage (allowing for the gain of the fluctuating signal) to reconstruct the actual instantaneous bridge voltages.

Temperature measurements were taken at each point in the wall jet flow where a cross-wire measurement was also to be taken. This allowed for the measured bridge voltages to be corrected for the local temperature, as described in Section 3.2.1. Each bridge voltage sample was corrected before it was converted into an instantaneous velocity using the calibration equation. These instantaneous velocities were the effective velocities normal to each wire.

The velocity components along and normal to the probe axis were found using the following relations: 25

$$
\begin{aligned}
& \mathrm{u}=\frac{\left(\sin \psi_{1}\right) \mathrm{U}_{\mathrm{eff}}-\left(\sin \psi_{2}\right) \mathrm{U}_{\mathrm{eff}}}{\left(\sin \psi_{1}\right)\left(\cos \psi_{2}\right)-\left(\cos \psi_{1}\right)\left(\sin \psi_{2}\right)} \\
& \mathrm{v}=\frac{\left(\cos \psi_{2}\right) \mathrm{U}_{\mathrm{eff}}-\left(\cos \psi_{1}\right) \mathrm{U}_{\mathrm{eff} 2}}{\left(\sin \psi_{1}\right)\left(\cos \psi_{2}\right)-\left(\cos \psi_{1}\right)\left(\sin \psi_{2}\right)}
\end{aligned}
$$

where $\psi_{1}$ and $\psi_{2}$ were the angles between each wire and the normal to the 
probe axis, and the angle $\psi_{1}$ was negative (fig. 3.6). The velocities were found for each instantaneous measurement, and subsequently, a statistical analysis was performed to determine the mean and fluctuating velocity components. Fig. 3.7 shows a flowchart of the cross-wire anemometry instrumentation.

\subsubsection{Digital Sampling of the AC Signal}

The first set of cross-wire measurements was made using the DEC-LAB $A / D$ converter on the PDP/11-23 minicomputer. The maximum sampling rate with this system was $1.25 \mathrm{kHz}$ for two-channel sampling. A repeat of the cross-wire measurements was made using an IBM AT personal computer, which provided two-channel sampling rates of up to $65 \mathrm{kHz}$ per channel.

An examination of the results from the earlier experiments using the DEC-LAB system showed some unexpected trends in the normal turbulent stress measurements. In wall jet flows, the peak $\overline{v^{\prime 2}} / \mathrm{U}^{2}$ should be about 608 of the peak $\overline{u^{\prime}{ }^{2}} / \mathrm{N}_{\mathrm{m}}^{2}$ (Ref. 4). Although this relationship held at the downstream sections of the flow, upstream $\left(\mathrm{v}^{\prime 2} \mathrm{~N}_{\mathrm{m}}{ }^{2}\right)_{\mathrm{max}}$ was measured to be higher than $\left(\overline{u^{\prime}} /_{\mathrm{m}}^{2}\right)_{\mathrm{max}}$. The normalized shear stress was lower than expected throughout the jet length.

It was initially believed that the low sampling rate was inadequate for capturing the high frequency fluctuations, although theoretically, the magnitude of the measured fluctuations should be independent of sampling frequency. When the IBM AT system was implemented, it was found that the above trends repeated at low sampling frequencies, but changed dramatically at higher sampling rates. At one upstream point in the flow, increasing the sampling rate per channel from $1 \mathrm{kHz}$ to 10 $\mathrm{kHz}$ increased $\overline{\mathrm{u}^{\prime 2}}$ by 388 and decreased $\overline{\mathrm{v}^{\prime 2}}$ by 308 . This trend supported the view that sampling rates may influence the magnitude of the velocity fluctuations.

Further experimentation revealed that the sampling rate per channel was not responsible for the error, but rather the time lag between samples of the two consecutive channels was the cause. The A/D converter was found to alternately step to each channel in sequence at 
a rate proportional to the given sampling rate. Therefore, at low sampling rates, the corresponding window in which the two channels were sampled was large enough to allow the bridge voltage to fluctuate. This caused a large error in the cross-wire measurements, since both bridge voltages are used to compute one velocity. It was necessary that the time lag between the two channels sampled be smaller than the time scales present in the flow.

An oscilloscope trace of the two AC voltage outputs from the bridges indicated that there were sharp spikes superimposed on the random fluctuating signal. If the spikes are correlated between the two wires, then a time lag between channel sampling would cause the measurement of $u$ (summation of the two voltages) to be undervalued, and the measurement of $v$ (difference between the two voltages) to be overvalued. This could account for the measurement of $\overline{u^{\prime 2}}$ being too low and $\overline{v^{\prime 2}}$ being too high at low sampling rates.

The IBM AT system allowed the sampling frequency to be increased up to $65 \mathrm{kHz}$ per channel. It was found experimentally that a sampling rate of $10 \mathrm{kHz}$ per channel was adequate to obtain stable velocity fluctuation levels. This sampling rate corresponded to a time lag between channels of $50 \mu \mathrm{sec}$. As the sampling rate was increased from 1 $\mathrm{kHz}$ to $10 \mathrm{kHz}, \overline{\mathrm{u}^{\prime 2}}$ increased and $\overline{\mathrm{v}^{2}}$ decreased to a steady-state value. Figure 3.8 shows the variation in the velocity fluctuation levels as the time lag between channels increases, corresponding to a decrease in sampling rate.

To find the frequencies occurring in the jet flow, a fast fourier transform (FFT) was performed on the velocity samples at several points in the flow (fig. 3.9). The FFT used 4096 samples of both the $u$ and $v$ component of velocity, and output the spectra for each. It was found that frequencies occurring in the flow were fairly broadband up to 20 $\mathrm{kHz}$, above which there was only low-level noise.

It was decided to take the cross-wire measurements at a sampling rate of $40 \mathrm{kHz}$ per channel, with a sample size of 30,000 data points per channel. The time lag between consecutive channels was thus only 12.5 $\mu \mathrm{sec}$, and the total duration of the sample was 0.75 seconds, which is long enough to resolve any flow frequencies above $3 \mathrm{~Hz}$. Since the $\mathrm{AC}$ 
signal had frequencies of less than $5 \mathrm{~Hz}$ filtered out, this sampling duration was more than adequate.

\subsubsection{Data Reduction}

Statistical quantities in the flow were computed on-line during the experiment. The 30,000 samples were taken on each channel, and were stored as integer $A / D$ output values. Blocks of 200 integer results were taken at a time (100 per channel) and converted to effective velocities for each wire. The integer output-to-velocity conversions had previously been stored in a lookup table, so repeat occurrences of $A / D$ output were not recomputed. The effective wire velocities were combined into $u$ and $v$ velocity components, and a running sum of the following quantities were computed: $\Sigma u, \Sigma v, \Sigma u^{2}, \Sigma v^{2}, \Sigma u v, \Sigma u^{2} v$, and $\Sigma u v^{2}$. This process was repeated with the next 100 samples from each channel. In this way, the computer storage requirement was minimized. The running sums were then normalized by the number of samples to give the mean values $s\langle u\rangle, s\langle v\rangle, s\left\langle u^{2}\right\rangle, s\left\langle v^{2}\right\rangle, s\langle u v\rangle$, $S\left\langle u^{2} v\right\rangle$, and $S\left\langle u v^{2}\right\rangle$, where $s\langle u\rangle-\Sigma u /$ (no. of samples). The turbulence quantities could be computed:

$$
\begin{aligned}
& \bar{u} \quad-s<u> \\
& \bar{v} \quad-s<v> \\
& \overline{u^{\prime 2}}-s<u^{2}>-s<u>S<u> \\
& \overline{v^{\prime}}-S\left\langle v^{2}\right\rangle-S<v>S<v> \\
& \overline{u^{\prime} v^{\prime}}-s\langle u v\rangle-s\langle u\rangle s\langle v\rangle \\
& \left.\left.\left.\overline{u^{\prime 2} v^{\prime}}-s<u^{2} v\right\rangle-2 s<u>s<u v\right\rangle-s<v>s\left\langle u^{2}\right\rangle+2 s<v>s<u>s<u\right\rangle \\
& \overline{u^{\prime} v^{\prime 2}}-s\left\langle u v^{2}\right\rangle-2 s\langle v\rangle s\langle u v\rangle-s\langle u\rangle s\left\langle v^{2}\right\rangle+2 s\langle u\rangle s\langle v\rangle s\langle v\rangle
\end{aligned}
$$

The data acquisition program plotted these quantities vs. $y$ during the data acquisition and stored the data to disk. Traversing the probes across the jet was also controlled by the computer, and twenty-one data points were taken in each jet profile. The program took approximately 
45 seconds per data point, including the 10 seconds for the sampling of the DC bridge voltages.

An off-line computer program was used to normalize the velocities with the local maximum velocity $U_{m}$ and the $y$-coordinates with the local jet half-width $b$, and plotted these non-dimensional profiles.

\subsubsection{Probe Limitations and Error Analysis}

Cross-wire anemometry is widely accepted for the measurement of turbulence. However, this technique can not hold claim to being accurate throughout the entire range of velocity gradients, frequencies, turbulence levels, and temperature variations present in the wall jet flow.

The main source of error in the cross-wire measurements was the poor spatial resolution of the probe in the thin jet region closest to the slot. At positions where the width of the probe ( $1 \mathrm{~mm}$ ) was $108-158$ of the jet half-width, velocity gradients would exist along the length of the wires. This problem was particularly relevant to this experiment due to the thin slot and high exit velocity, and is not a problem inherent in the method in general.

Additional errors in the cross-wire measurements could be caused by slight calibration errors, drift, and temperature variations. Fitting curves through the calibration data produced a 1.58 error for each hot wire. Drift between calibration checks could not be estimated. The cross-wire was fairly stable, and the calibration remained constant, with the exception of the wire resistance changing on a daily basis.

In order to account for temperature effects in the jet, temperature profiles were taken after the blower had been running long enough for the plenum to heat up to an equilibrium level. All cross-wire measurements were also taken after the model had warmed up. The room temperature remained constant from day to day, as did the equilibrium plenum temperature. Thus, it was unlikely that any local jet temperature varied by more than $0.5^{\circ} \mathrm{C}$ from the time the temperature measurements were taken to the time the hot wire measurements were taken. The temperature correction was valid for small changes between measurement 
and calibration temperature, which applied over most of the jet. Once the velocities were normalized, temperature should account for an error of no more than 38 , and probably much less.

It was found, while performing the yaw calibration on the cross-wire probe, that the wires were typically not at $45^{\circ}$ to the probe axis. For the cross-wire probe used, each wire was $42^{\circ}$ from the normal to the probe axis. Since eqs. (3.4) become slightly more complex with these angles, it was decided not to account for tangential flow cooling over the wires. The equations which account for the tangential cooling velocity in addition to the normal velocity become intractable unless it is assumed that the wires are at $45^{\circ}$ to the probe axis. Since tangential cooling causes an error typically less than 48, it was decided to neglect tangential cooling in favor of including the correct wire angles. In certain instances, it was necessary to tilt the probe in the $u-v$ plane in order to take measurements close to the wall, and eqs. (3.4) allowed the tilt angle to be accounted for in the calibration.

In the outer layer of the jet, entrained fluid may cause the mean velocity vector to vary significantly in direction from the cross-wire probe axis. This causes the hot-wire measurements to be unreliable in the outer shear layer. The normalized turbulent stresses displayed an average of 58 scatter at the peak intensity locations when the data runs were repeated. There was less scatter away from the peak intensity locations.

Another source of error was in the measurement of $y$, the normal coordinate to the wall. Since the probes could not actually touch the wall, pin gauges and a magnifying glass were used to position the wire prongs a set distance from the wall. An offset of $0.5 \mathrm{~mm}$ was added to this distance to account for the width of the probe and to make the initial $\mathrm{y}$-measurement correspond to the center of the four prongs. Any errox in determining the distance of the probe from the wall would be small compared to the error in pinpointing the measurement location within the $1 \mathrm{~mm}$ probe width, since the distance of the nearest prong to the wall was typically less than one quarter of a millimeter. 


\subsubsection{LDV System}

One objective of this experiment was to take turbulence measurements using a two-component Laser Doppler Velocimetry (LDV) system. It was felt that an LDV system could measure the high turbulence intensities present in wall jet flows, while avoiding the problems with calibration, temperature, and spatial resolution particular to crosswires. With no probe interference in the flow and with an improved spatial resolution, it was possible to take measurements in thinner jet flows, providing improved two-dimensionality for an annular configuration. Also, the use of a cylindrical model alleviated the problems associated with taking LDV measurements near a wall.

The optical system used a 4-watt Argon Ion laser (fig. 3.10). The output beam was split into blue $(488 \mathrm{~nm})$ and green $(514.5 \mathrm{~nm})$ beam pairs. One beam from each pair was shifted by a fixed Bragg frequency of $40 \mathrm{Mhz}$. The 4-beam matrix was then sent to a transmitting optics table. At this table, mirrors and lenses were mounted on a threedimensional traversing mechanism. A lens focused the four beams at the measuring volume. The receiving optics were in the off-axis forward scatter mode, and were mounted on a traversing gear which moved synchronously with the transmitting optics. Lenses and filters were used to separate the scattered light into blue and green, and this light was focused onto two photomultiplier tubes.

The signals from the photomultiplier tubes were amplified and relayed to the signal processors (fig. 3.11). The signals were then mixed electronically with two sine waves. The mixing procedure was necessary for low-speed flows, where the actual Doppler frequencies were small compared to the Bragg frequency of $40 \mathrm{Mhz}$. In order to reduce the effective measured frequency and hence improve the counter resolution, the incoming signals were mixed with sine waves of known frequency.

The mixed signals were fed into single particle burst counters which measured the zero crossings of the Doppler signal. The processors used two checks to validate a Doppler signal. The first check was the usual $5 / 8$ comparison, where the processor checks the frequency for 5 zero crossings against that for 8 crossings. The second check was the 
multi-sequence check. Positive and negative thresholds were set on the signal, and a validated output was permitted only if, for all eight fringe crossings, the signal passes through a positive threshold, a zero level, and a negative threshold in the proper sequence. The digital data was then passed to the computer interface.

The computer interface was used to transfer the data from the signal processors to an HP 9845B desktop computer. The computer interface can accept data from the processors in either random or sync mode. In random mode, data would be accepted whenever an event occurred on either of the channels. In sync mode, data would only be accepted when both processors sample simultaneous events. This was the mode necessary to take coincident data for shear stress measurements. A coincidence window of $5 \mu \mathrm{sec}$ was set on the computer interface during sync mode, which was the smallest window setting available. This was comparable to the time lag used in the cross-wire experiment, which was $12.5 \mu \mathrm{sec}$ at a sampling rate of $40 \mathrm{kHz}$ per channel. Multiplexed data was passed to the computer. A detailed description of the optics and the signal processing hardware used in this work can be found in an earlier work. 26

A data acquisition program sampled 2000 data points from each channel, and stored the data to floppy disk. This program had a limited capability for on-line data reduction, so that key results could be monitored during a run. An off-line program was used to fully reduce the data. Filtering of the data was used to reduce some of the spurious noise present in the measurements. The standard deviation of the velocity was calculated, and any data samples greater than 2.5 standard deviations from the mean were discarded. The mean and new standard deviation were then recalculated. This program also plotted histograms at each data point for each channel, and plotted the turbulence profiles. Statistical quantities were computed as described earlier in Sec. 3.2.4. Ref. 26 contains a complete description of the software used for this system, as well as program listings.

Seeding of the flow was accomplished using smoke particles from burning mineral oil. The smoke was input directly into the air intake of the blower. 


\subsubsection{LDV Limitations}

It was difficult to quantize the errors present in the LDV measurements. The signal:noise ratio was dependent on many different things, such as the laser power, the sensitivity of the PM tubes, the PM tube aperature area, the off-axis angle of the receiving optics, the alignment of the beams in the measuring volume, and the seeding. In this particular system, beam drift which was possibly caused by vibrations from the blower had led to misalignment in the probe volume. The shift in the beams contributed largely to a loss of coincident data rate and also caused the measurement volume to shift position, which led to an inaccuracy in the measured $y$-coordinate.

A bias was also present in the measurements, since the ambient air could not be seeded. Thus, fluid originating from the plenum would have a higher probability of being detected than fluid that was entrained from the surroundings. This bias may be alleviated in a wind tunnel test of a wall jet with an external stream.

During the course of this experiment, it was noticed that the green beam pair, measuring the u-component of velocity, was more susceptible to drift and a higher noise level than the blue beam pair (v-component). The u-component measurements tended to have more scatter and were less repeatable than the v-component measurements. The noise threshold setting was adjusted to reduce these noise levels. The threshold was usually set so that the data rate was about half of the data rate at zero threshold. Another check was to block one beam in each color pair. The data rate on the corresponding channel should be zero when one beam is blocked. Ideally, in any flow with a low-turbulence freestream, the threshold can be set such that histograms taken in the freestream display a sharp, narrow peak. Unfortunately, no freestream was available in this experiment, so a proper determination of the threshold setting was not easily made. 


\section{RESULTS}

\subsection{Mean Flow}

Straight wall jets achieve a self-similar velocity profile if the velocity and distance from the wall are normalized by a characteristic velocity scale and length scale, respectively. These characteristic scales are the local maximum velocity and the jet half-width. The jet half-width is defined as the distance from the wall where the velocity is equal to half the maximum velocity. Figure 4.1 defines the nomenclature for both straight and curved wall jets. The slot height is $h$, and the constant fet exit velocity is $U_{j}$. Downstream, the jet achieves a fully developed velocity profile, with the maximum velocity $U_{m}$ and the half-width, denoted by $b$. The streamwise and normal coordinates are $x$ and $y$, respectively. In the curved wall jet, $x$ and $y$ are curvilinear. Also in the curved wall jet, the streamwise radius of the wall is denoted by $R$. The ratio $h / R$ is used to signify the strength of the streamwise curvature and is constant for circular arc walls. The ratio $b / R$ may be used as a local curvature parameter, since it varies downstream as the jet grows.

The important mean flow quantities measured in wall jet experiments are the changes in $U_{m}$ and $b$ in the streamwise direction. The maximum velocity will decay and the jet half-width will grow as the jet entrains the surrounding fluid. Mean velocity profiles are also measured to test for self-similarity in the straight and curved wall jets.

For each of the three surfaces tested, the first set of experiments was to determine the flow uniformity about the circumference. Simple total head pitot tubes were used for these tests. For the straight wall jet, the total pressure may be directly related to the jet velocities, since one can make the boundary layer assumption that the static pressure in the jet is equal to the ambient room pressure. However, in curved flows, a pressure gradient exists across the jet, due to centrifugal force, and it is no longer simple to relate the total pressure to the velocity. For the curved jets, the pitot 
tube was used to check flow uniformity, but the jet velocities where not computed from these measurements.

Mean velocity profiles of the straight wall flowfield were measured with pitot probes at several positions about the circumference of the cylinder. These results showed good flow uniformity and "two-dimensionality" along the jet span (fig. 4.2). The maximum velocity decay rate was found to be very sensitive to flow nonuniformities along the span, and this could be used as an indicator of flow quality. Both a two-dimensional and an axisymmetric momentum equation check were used to assess two-dimensionality. 4 In a two-dimensional flow, loss of streamwise momentum should be small since it is due only to skin friction. Thus, the ratio of jet momentum $M(x)$ relative to the slot momentum $M_{0}$ is

$$
\frac{M(x)}{M_{0}}=\left(\frac{U_{m}}{U_{j}}\right)^{2} \frac{b}{h} \int_{0}^{\infty}\left(\frac{U}{U_{m}}\right)^{2} d \xi
$$

For an axisymmetric flow:

$$
\frac{M(x)}{M_{0}}=\left(\frac{U_{m}}{U_{j}}\right)^{2} \frac{b}{h} \int_{0}^{\infty} \frac{r}{r_{0}}\left(\frac{U}{U_{m}}\right)^{2} d \xi
$$

where $r$ is the annular radial coordinate and $r_{0}$ indicates the surface of the model. In a frictionless 2 - $d$ flow, $M(x) / M_{0}=1$ for all $x$. In actuality, the ratio decreases slowly with distance downstream. For this flow, using the $2-d$ equation, the momentum ratio ranged from .89 to .76 , and with the axisymmetric equation, the ratio ranged from .90 to .77 . The difference was less than 38 between the two calculations, and they both showed adequate conservation of momentum in the streamwise direction. Jet width growth rates and velocity decay rates agreed well with results obtained by previous researchers, and were given by:

$$
\begin{aligned}
& \mathrm{db} / \mathrm{dx}=.074 \\
& \mathrm{U}_{\mathrm{m}} / \mathrm{U}_{\mathrm{j}}=4.9(\mathrm{x} / \mathrm{h}+9.6)^{-.55}
\end{aligned}
$$


The mean velocity profiles measured using a pitot probe exhibited the self-similar shape typical of plane wall jets after 15 slot heights downstream (fig. 4.3).

Pitot profiles of the two curved wall jets also displayed good flow uniformity along the span. For each model, wool tufts and oil flow were used to visualize the streamlines, and in each case, the streamlines were all axial along the cylinder, with no indication of swirl present.

Mean velocity measurements in both the straight and curved wall jets were taken with cross-wire anemometry, as described in the previous chapter. Figures 4.4 through 4.7 show the mean velocity profiles for each of the three models. Figure 4.4 shows the LDV measurements in the straight annular model, and figs. 4.5 through 4.7 are cross-wire measurements for the three models. The profiles measured with the LDV showed more scatter than the corresponding profiles measured with the cross-wire. The two-dimensional straight wall jet is a self-similar flow, and the profiles in fig. 4.5 indicated that the annular straight wall jet was also self-similar. The dotted line shows a two-dimensional velocity profile from Ref. 18, which was representative of previous straight wall jet experiments. There was virtually no difference between the current annular profiles and the previous two-dimensional profiles.

Curved wall jets are self-similar only if the ratio of the wall radius of curvature is proportional to the distance downstream, as with a logarithmic spiral. Although wall jets over circular arcs (constant radius of curvature) are not strictly self-similar, the mean flow changes slowly enough downstream that the self-similar approximation is valid. The mildly curved wall jet (fig. 4.6) had velocity profiles which were identical to the straight wall case. Only for the highly curved wall jet (fig. 4.7) could any variation in the velocity profiles be seen with downstream distance, but the change was still slight. Figure 4.7 also shows a comparison with the data for the straight wall jet model. The highly curved velocity profile had a slightly fuller profile above the velocity maximum. This difference was seen in two-dimensional wall jets as well. 18 
The growth of the wall jet half-width, $b$ and the decay of the local jet maximum velocity, $U_{m}$ are nearly universal within two-dimensional wall jet flows. The half-width growth rate was almost invariant amongst previous researchers, but the maximum velocity decay had a slight dependence on Reynolds number. Figure 4.8 shows the straight annular wall jet growth rate plotted with two-dimensional data from previous researchers. Except for a shift in the virtual origin which varies from experiment to experiment, the annular jet grew at the same rate as previously measured rectangular jets. Figure 4.9 shows a similar plot for the straight annular wall jet maximum velocity decay rate. Reynolds number effects were apparent, and again, the annular jet fitted in well with rectangular jet measurements.

Similar plots of the jet growth and velocity decay rates for curved wall jets are shown in figs. 4.10 and 4.11. Curved wall jets display enhanced mixing relative to straight wall jets, and this is shown by the increased growth rates of these jets. As the curvature of the jet increases, the growth rate also increases. Correspondingly, the decay rate of the maximum velocity increases as well. The annular data showed the same trends as previous two-dimensional results.

\subsection{Turbulence Results}

The turbulence quantities of interest in wall jet flows are the Reynolds stresses, in particular the shear stress, $\overline{u^{\prime} v^{\prime}} / \mathrm{v}_{\mathrm{m}}{ }^{2}$. These stresses are normalized by the local mean maximum velocity. Although the normal stresses $\overline{\mathrm{u}^{\prime 2}} / \mathrm{U}_{\mathrm{m}}{ }^{2}$ and $\overline{\mathrm{v}^{\prime 2}} / \mathrm{U}_{\mathrm{m}}{ }^{2}$ are also of interest, the shear stress is the term which needs to be modeled in a calculation scheme.

Turbulence results were obtained using cross-wire anemometry and Laser Doppler Velocimetry. The cross-wire measurements make up the majority of the experimental results, with measurements of the three Reynolds stresses and two triple components made in all three flow cases. The 2-component LDV system was available on a limited basis, so only measurements in the straight wall jet case were made. The three Reynolds stresses and two triple components were measured with the LDV. 
Measurements of the three Reynolds stresses were plotted for the straight wall model in figs. 4.12 through 4.23 , showing both cross-wire and LDV results. Figures 4.12 and 4.13 show the streamwise normal stress $\mathrm{u}^{\prime 2} / \mathrm{J}_{\mathrm{m}}^{2}$ profiles as they varied with downstream distance. The LDV measured consistently higher $\overline{\mathrm{u}^{\prime 2}} / \mathrm{U}_{\mathrm{m}}{ }^{2}$ profiles than did the crosswire. Since there was so much noise on the u-channel in the LDV system, it was believed that the higher turbulence intensities measured were due to noise in the system and a bias away from measuring the slower entrained fluid, rather than a turbulence signal. The normalized streamwise turbulence intensity increased slightly downstream, indicating that the turbulence quantities have not reached a selfpreserving state. This trend can be found in previous work as well.18 Near the wall, the streamwise turbulence intensity reached a minimum and then increased again very close to the wall. The secondary intensity peak was due to the boundary layer flow, where the velocity gradient was very large. The LDV was able to measure the minimum point better than the cross-wire, since it had better spatial resolution and could be used closer to the wall. The cross-wire only measured the intensity minimum at far downstream stations, where the jet was very thick relative to the probe width. Neither probe could get close enough to the wall to measure the decrease in $\overline{u^{\prime 2}}$ which must occur as the wall is reached. Figure 4.12 also shows a bandwidth of previous two-dimensional data. The annular wall jet profiles agreed with the two-dimensional profiles in magnitude and shape. The cross-wire and LDV measurements are compared directly in fig. 4.14 at one downstream station. The growth of the peak value of $\overline{u^{\prime 2}} \mathrm{~J}_{\mathrm{m}}{ }^{2}$ is plotted in fig. 4.15 for the present data and for previous two-dimensional data. The slight growth in the peak normal stress could be seen in all cases, indicating that this parameter did not achieve self-similarity until far downstream.

Figures 4.16 and 4.17 show the cross-wire and LDV measurements of the normal turbulence intensity $\overline{v^{\prime 2}} \mathrm{~N}_{\mathrm{m}}{ }^{2}$ profiles as they changed downstream. The cross-wire results showed a collapse of these profiles, indicating that this quantity was more self-similar than $\overline{\mathrm{u}^{\prime}} \mathrm{J}_{\mathrm{m}}{ }^{2}$. However, the LDV results clearly showed the $\overline{v^{\prime 2}} N_{m}^{2}$ profiles growing 
with downstream distance. Figure 4.16 shows a bandwidth which represents the range of measurements taken by previous researchers. of all the turbulence parameters measured, the quantity $\overline{\mathrm{v}^{2}} / \mathrm{J}_{\mathrm{m}}^{2}$ showed the least agreement among researchers, with the peak magnitude varying by as much as a factor of two. The present annular results fell within this bandwidth, and a comparison of the cross-wire and LDV results illustrated the difficulties that existed in measuring this particular quantity.

The near-wall behavior of $\overline{v^{\prime 2}}$ shows the normal stress falling to zero at the wall, in contrast to ${\mathrm{u}^{\prime 2}}^{2}$. The wall damped the v-fluctuations in the boundary layer region, although the high rate of strain near the wall caused a production of ${\mathrm{u}^{\prime 2}}^{2}$. Figure 4.18 directly compares cross-wire and LDV results at one downstream station. Figure 4.19 shows the peak $\overline{\mathrm{v}^{\prime 2}} \mathrm{~N}_{\mathrm{m}}^{2}$ as it changes downstream.

Figures 4.20 and 4.21 show the Reynolds shear stress profiles as they changed downstream for the annular straight wall jet. The cross-wire results and the LDV results were in agreement, to some extent. The profiles were nearly self-similar with downstream distance, with only a slight growth seen. Figure 4.20 also shows a bandwidth indicating previous results. The profile shapes agreed with previous two-dimensional data; however, the peak shear stress in the present experiment was in all cases lower than the peak shear obtained by previous researchers. The near wall behavior of the shear stress, seen in figs. 4.20 and 4.21 , showed the well-documented effect that the position of zero shear stress occurred below the position of zero mean velocity gradient. The cross-wire and LDV profiles are compared in fig. 4.22 at one downstream station. Figure 4.23 shows the growth of the peak shear stress with downstream distance, and although the growth rate of the annular data agreed with two-dimensional data, the peak was seen to be lower.

Reynolds stress profiles for the curved wall jets are shown in figs. 4.24 through 4.29. These profiles were all taken using cross-wire anemometry. These plots demonstrated that the degree of self-similarity of the normalized turbulent quantities decreased as the streamwise curvature increased. The results for the mildly curved wall jet were 
similar to those of the straight wall, although a slightly higher growth of the peak intensities could be seen. In the highly curved wall jet, this growth was more clearly indicated, showing that although the mean flow was close to self-similar, the turbulence field was not. Figures 4.30 through 4.32 show the growth rate of the peak stresses against downstream distance, and compares these with the straight wall case.

Triple turbulence component profiles for all three flows are shown in figs. 4.33 through 4.35. Triple products represent the turbulence transport of the Reynolds stresses. In all three wall jet flows, the triple product profiles were invariant with downstream position, which would simplify the task of modeling these terms in the Reynolds stress transport equations.

The results of the experimental investigation have shown the effects of streamwise curvature on the mean and turbulent wall jet flowfield, and these effects are summarized here. The mean velocity profiles were found to be invariant with downstream location for both the straight and curved wall jets, although the highly curved jet profile was slightly fuller near the velocity maximum. Curvature strongly affected the growth rate of the jet half-width, causing it to increase with higher curvature. There did not seem to be much appreciable difference between the annular mean flow data and data from previous rectangular experiments.

The Reynolds stress profiles showed self-similarity in the straight wall jet, but the turbulence quantities were increased relative to the mean flow in the curved jets. This same trend was seen for all three Reynolds stresses. The Reynolds stress profiles taken in the annular models followed similar trends to previous data taken in rectangular models. The one exception was the magnitude of the peak shear stress, which was slightly lower in the annular experiment. 


\section{ANALYSIS}

\subsection{Formulation}

An integral analysis was developed to predict the effects of transverse curvature on the mean flow and turbulent shear stress in curved wall jets. The analysis was originally developed for two-dimensional curved wall jets, 27 and was extended to the axisymmetric case of a wall jet over a curved body of revolution.

Integral methods are used in flows in which a self-similar velocity profile may be assumed. Since the normalized velocity profile can be easily expressed in functional form, the momentum equation may be integrated directly to obtain the shear stress. For wall jets, the self-similar velocity profile is obtained by normalizing the velocities and lengths by the local maximum velocity $U_{m}(x)$ and the local jet half-width $b(x)$, respectively. Thus, it is necessary to solve for the downstream development of both $U_{m}$ and $b$ in order to completely determine the mean velocity field in the flow. Two equations were found in terms of the jet growth rate, $d b / d x$, and the rate of change of the momentum flux, $d\left(b U_{m}{ }^{2}\right) / d x$. A marching scheme was used to solve these differential equations along the streamwise direction, thus allowing the solution of $U_{m}, b$, and the shear stress profile to be found at each $\mathrm{x}$-location.

The thin shear layer equations of motion for a wall jet over a body of revolution were used. 28 The differential form of these equations was:

$$
\begin{array}{cc}
\frac{\partial(r u)}{\partial x}+\frac{\partial(r v)}{\partial y}=0 & \text { mass } \\
u \frac{\partial u}{\partial x}+v \frac{\partial u}{\partial y}=-\frac{1}{\rho} \frac{\partial p}{\partial x}+\frac{1}{\rho r} \frac{\partial(r \tau)}{\partial y} & x \text {-momentum } \\
\frac{u^{2}}{R}=\frac{1}{\rho} \frac{\partial p}{\partial y} & y \text {-momentum }
\end{array}
$$

In this notation, $x$ and $y$ are curvilinear coordinates which follow the 
wall, and $r$ is the distance to the axis of revolution (fig. 5.1):

$$
r(x, y)=r_{0}(x)+y \cos \phi(x)
$$

For a straight wall, $r_{0}$ is constant, and $\phi$ is everywhere equal to zero, so $r$ is a function of $y$ only. If then the jet is thin compared to the radius of the cylinder, $d\left(r / r_{0}\right) / d y$ is approximately zero, and the equations reduce to the 2-d plane equations. For curved flows, $R$ is the local streamwise radius of curvature, as in the two-dimensional equations. In the following analysis, $r$ was kept as a function of $x$ and $y$, and $R$ was an arbitrary function of $x$. The jet was assumed to be thin compared with both the streamwise and annular radii of curvature, so terms $O(b / R)^{2}$ and $O\left(b / r_{0}\right)^{2}$ were neglected.

The integral forms of the equations of motion are given by:

$$
\begin{array}{ccc}
v=-\frac{1}{r} \int_{0}^{y} \frac{\partial(r u)}{\partial x} d y & \text { mass } \\
\int_{y}^{\infty} \frac{1}{r_{0}} \frac{\partial\left(u^{2} r\right)}{\partial x} d y-\frac{r}{r_{0}} u v=\int_{y}^{\infty}-\frac{1}{\rho} \frac{\partial p}{\partial x} d y-\frac{r}{r_{0}} \frac{r}{\rho} & \text { x-momentum } \\
\frac{P}{\rho}=-\int_{y}^{\infty} \frac{u^{2}}{R} d y & \text { y-momentum }
\end{array}
$$

By letting $h=r / r_{0}$ and combining (5.1) and (5.2), the integral momentum equation can also be written:

$$
\int_{y}^{\infty} \frac{1}{r_{0}} \frac{\partial\left(u^{2} r\right)}{\partial x} d y-\int_{y}^{\infty} \frac{\partial(h u v)}{\partial y} d y=\int_{y}^{\infty}-\frac{1}{\rho} \frac{\partial p}{\partial x} d y+\int_{y}^{\infty} \frac{1}{\rho} \frac{\partial(h r)}{\partial y} d y
$$

\subsection{Self-Similar Mean Velocity Profile}

The assumed self-similar velocity profile was given by the following functions: 


$$
\begin{aligned}
& \frac{\mathrm{U}}{\mathrm{U}_{\mathrm{m}}}=2\left(\frac{\xi}{\xi_{\mathrm{m}}}\right)^{1 / \mathrm{n}}-\left(\frac{\xi}{\xi_{\mathrm{m}}}\right)^{2 / \mathrm{n}}=\mathrm{f}(\xi) \quad \xi \leq \xi_{\mathrm{m}} \\
& \frac{U}{U_{m}}=\operatorname{sech}^{2} \frac{\kappa\left(\xi-\xi_{m}\right)}{\left(1-\xi_{m}\right)} \quad-f(\xi) \quad \xi \geq \xi_{m}
\end{aligned}
$$

where $\xi-y / b$. The first function gave the velocity profile for the inner region of the jet. The power law form was suggested by the relation for turbulent boundary layers. Typical values of the exponent $\mathrm{n}$ for boundary layer flows were 6 or 7 . The second relationship, for the outer region of the flow, came directly from the solution for a turbulent two-dimensional free jet. Both of the above functions have been written so that the maximum velocity occurred at $\xi-\xi_{m}$.

By setting $U / \mathrm{U}_{\mathrm{m}}=1 / 2$ at $\xi=1$, the constant $\alpha$ was found to be 0.8814 . Matching the second derivatives of the functions at $\xi-\xi_{m}$ and using n-6 gave the value $\xi_{m}=.159$, which was constant for all downstream locations. Figure 5.2 shows the assumed velocity profile, along with experimental data for the straight wall jet. The two profiles agreed very well everywhere except for a small region above the velocity maximum, where the assumed profile was slightly fuller than the experimental profile.

\subsection{Mean Flow Development}

The two equations necessary to solve for the growth rate and the rate of change of momentum flux were obtained by evaluating (5.8) at $\xi=0$ and by evaluating (5.2) at $\xi=\xi_{\mathrm{m}}$.

In (5.8), the pressure term was written as

$$
\begin{aligned}
-\frac{1}{\rho} \frac{\partial p}{\partial x} & =-\frac{1}{\rho} \frac{\partial p}{\partial x}-\frac{\partial\left(y u^{2} / R\right)}{\partial x}+\frac{\partial\left(y u^{2} / R\right)}{\partial x} \\
& =-\frac{\partial}{\partial y} \frac{\partial(y p / \rho)}{\partial x}+\frac{y}{R} \frac{\partial u^{2}}{\partial x}-y \frac{u^{2}}{R^{2}} \frac{d R}{d x}
\end{aligned}
$$

using (5.3). The substitution of (5.4) and (5.9) resulted in 


$$
\begin{aligned}
& \left(\frac{1}{\mathrm{U}_{\mathrm{m}}^{2}} \frac{\mathrm{d}\left(\mathrm{bU}_{\mathrm{m}}^{2}\right)}{\mathrm{dx}}+\frac{\mathrm{b}}{\mathrm{r}_{0}} \frac{\mathrm{dr_{0 }}}{\mathrm{dx}}\right) \mathrm{A}_{\mathrm{n}} \\
& +\left(\cos \phi-\frac{\mathrm{r}_{0}}{\mathrm{R}}\right)\left(\frac{1}{\mathrm{U}_{\mathrm{m}}^{2}} \frac{\mathrm{d}\left(\mathrm{bU}_{\mathrm{m}}{ }^{2}\right)}{\mathrm{dx}}+\frac{\mathrm{db}}{\mathrm{dx}}\right) \frac{\mathrm{b}}{\mathrm{r}_{0}} \mathrm{C}_{\mathrm{n}} \\
& +\left(\frac{\mathrm{r}_{0}{ }^{2}}{\mathrm{R}^{2}} \frac{\mathrm{dR}}{\mathrm{dx}}-\mathrm{r}_{0} \sin \phi \frac{\mathrm{d} \phi}{\mathrm{dx}}\right) \frac{\mathrm{b}^{2}}{\mathrm{r}_{0}{ }^{2}} C_{\mathrm{n}}=-\frac{1}{2} C_{f}
\end{aligned}
$$

where $\quad A_{n}=\int_{0}^{\infty} f^{2} d \xi=\frac{1+2 / 3 n}{1+\kappa n}$

$$
C_{n}=\int_{0}^{\infty} \xi f^{2} d \xi=\frac{\frac{1}{2}\left[\left(\frac{4}{3} \ln 2-\frac{1}{3}\right) n^{2}+\frac{4}{3} n+1\right]}{(1+\kappa n)^{2}}
$$

Assuming $n$ was large and $C_{f} \rightarrow 0$ (valid for large Reynolds number flows) gave

$$
\begin{aligned}
& A_{n}=\frac{2}{3 \kappa} \\
& C_{n}=\frac{2}{3 \kappa}\left[\frac{\ln 2-1 / 4}{\kappa}\right] \\
& {\left[1+\left(\cos \phi-\frac{r_{0}}{R}\right)\left(\frac{b}{r_{0}}\right)\left(\frac{\ln 2-1 / 4}{\kappa}\right)\right] \frac{1}{U_{m}^{2}} \frac{d\left(b U_{m}^{2}\right)}{d x}} \\
& +\left[\left(\cos \phi-\frac{r_{0}}{R}\right) \frac{b}{r_{0}}\left(\frac{\ln 2-1 / 4}{\kappa}\right)\right] \frac{d b}{d x}+\frac{b}{r_{0}} \frac{d r_{0}}{d x} \\
& +\left(\frac{r_{0}^{2}}{R^{2}} \frac{d R}{d x}-r_{0} \sin \phi \frac{d \phi}{d x}\right)\left(\frac{\ln 2-1 / 4}{\kappa}\right) \frac{b^{2}}{r_{0}^{2}}=0
\end{aligned}
$$

Evaluating (5.2) at $\xi-\xi_{\mathrm{m}}$ required the use of an eddy viscosity approximation for the value of $\tau$ at $\xi_{\mathrm{m}}$. Setting

$$
\begin{aligned}
\tau / \rho & =\epsilon\left(\frac{\partial u}{\partial y}-\frac{u}{R}\right) \\
\epsilon & =\frac{K}{4 \kappa^{2}}\left(1-\xi_{m}\right) b U_{m} g(\xi) \\
g(\xi) & =\left(\xi / \xi_{m}\right)^{m}\left(a+(1-a) \xi / \xi_{m}\right)
\end{aligned}
$$

it was found that

$$
\mathrm{m}=1-1 / \mathrm{n}
$$


and

$$
a=C_{f} \kappa / K
$$

in order to satisfy the condition that the shear stress equals the skin friction at the wall. Assuming $n$ was large and $C_{f} \rightarrow 0$ gave the final equation

$$
\begin{aligned}
\frac{d b}{d x}= & K\left[1+\frac{b}{R} \frac{n}{\kappa}\right]+\frac{K}{2 \kappa^{2}} \cos \phi \frac{b}{r_{m}} \frac{b}{R} \\
& +\left(1-\frac{4}{3 \kappa} \frac{b}{R}\right) \frac{1}{U_{\infty}^{2}} \frac{d\left(b U_{m}^{2}\right)}{d x}+\frac{4}{3 \kappa} \frac{b^{2}}{R^{2}} \frac{d R}{d x}
\end{aligned}
$$

For a circular arc in the streamwise direction, $R$ - constant, and (5.12) and (5.13) were simplified by substituting:

$$
\begin{aligned}
& \frac{d \phi}{d x}=\frac{1}{R} \\
& \phi=\frac{x}{R} \\
& r_{0}(x)=r_{0}(0)-R(1-\cos \phi) \\
& \frac{d r_{0}}{d x}=-\sin \phi \\
& \frac{l n 2-1 / 4}{x}=.503 \\
& \cos \phi-\frac{r_{0}}{R}=1-\frac{r_{0}(0)}{R}
\end{aligned}
$$

The equations were now written:

$$
\begin{gathered}
\left(A \frac{b}{r_{0}}\right) \frac{d b}{d x}+\left(1+A \frac{b}{r_{0}}\right) \frac{1}{U_{m}^{2}} \frac{d\left(b_{m}^{2}\right)}{d x}=-\frac{b}{r_{0}} \frac{d r_{0}}{d x}\left(1+\frac{b}{R} c\right) \\
\frac{d b}{d x}+\left(\frac{4}{3 x} \frac{b}{R}-1\right) \frac{1}{U_{m}^{2}} \frac{d\left(b U_{m}^{2}\right)}{d x}=k\left(1+\frac{b}{R} \frac{n}{x}\right)
\end{gathered}
$$

where $C=C_{n} / A_{n}=(\ln 2-1 / 4) / \kappa=.503$ and $A=C\left(1-r_{0}(0) / R\right)$.

For the simpler case of a plane axisymmetric wall jet or a 2 -d curved wall jet, the functions $d b / d x$ and $1 / U_{m}{ }^{2} d\left(b U_{m}^{2}\right) / d x$ were found in closed form: 
axisymmetric plane

$$
\begin{aligned}
& \frac{d b}{d x}=k\left(1-c \frac{b}{r_{0}}\right) \\
& \frac{1}{U_{m}^{2}} \frac{d\left(b U_{m}^{2}\right)}{d x}=-c \frac{b}{r_{0}} K
\end{aligned}
$$

2-d curved ( $R$ - constant)

$$
\frac{1}{U_{m}^{2}} \frac{d\left(b U_{m}^{2}\right)}{d x}=0
$$

or

$$
\begin{aligned}
& \left(\frac{U_{j}^{2}}{U_{m}^{2}}\right)=\frac{b}{h} \\
& \frac{d b}{d x}=K\left(1+\frac{n}{x} \frac{b}{R}\right)
\end{aligned}
$$

The empirical constant $K$ is the $2-d$ plane wall jet growth rate, and was taken to be equal to 0.073 as suggested by previous research. 4

Equation (5.15) reduced to the two-dimensional relation (5.19) if $1 / \mathrm{U}_{\mathrm{m}}^{2} \mathrm{~d}\left(\mathrm{bU}_{\mathrm{m}}^{2}\right) / \mathrm{dx}=0$. In both equations, the rate of growth of the jet half-width had the same functional dependence on $b / R$. For a straight wall $(b / R=0)$, the growth rate was linear and equal to the constant $K$. The ratio $\mathrm{n} / \boldsymbol{\kappa}$ was approximately equal to 7 , which was almost two orders of magnitude higher than $K$. Thus, the curvature term dominated the growth rate, even for small b/R. Equation (5.19) had an exact solution for $b$ :

$$
\frac{b}{h}=\frac{R}{h} \frac{\kappa}{n}\left[\exp \left(K \frac{n}{\kappa} \frac{x-x_{0}}{h} \frac{h}{R}\right]-1\right]
$$

which showed that curved wall jets grow at an exponential rate, compared with a linear growth for a straight wall jet.

Equation (5.14) contained all of the transverse curvature effects in the mean flow equations. The transverse curvature appeared as the two quantities $b / r_{0}$ and $d r_{0} / d x$. As $b / r_{0} \rightarrow 0$, equation (5.14) would reduce to the two-dimensional case. 
Eqs. (5.14) and (5.15) were put into matrix form and solved for the two unknowns, $d b / d x$ and $1 / \mathrm{v}_{\mathrm{m}}{ }^{2} \mathrm{~d}\left(\mathrm{bU}_{\mathrm{m}}{ }^{2}\right) / d x$. The matrix equation was solved numerically by marching in the downstream direction. With the lengths $b$ and $x$ normalized by the slot height $h$, and $U_{m}{ }^{2}$ normalized by the exit velocity squared $U_{j}^{2}$, the starting conditions were taken to be

$$
\frac{U_{m}^{2}}{U_{j}^{2}}=1 \quad \text { @ } x / h=0
$$

and

$$
\mathrm{b} / \mathrm{h}=1 \quad \text { a } \mathrm{x} / \mathrm{h}=0
$$

The results for the mean flow computations are shown in figs. 5.3 through 5.6 for both annular and two-dimensional flow. Data is also presented as a comparison to the analysis. The effect of streamwise curvature on the growth rates was clearly seen in both annular and two-dimensional flow (figs. 5.3 and 5.4). The growth of the twodimensional jet half-width was highly dependent on the streamwise curvature, due to centrifugal force effects on the mixing of the jet with the surrounding fluid. The analytical results, shown for $c_{f}=0$, agreed with experimental results for both rectangular and annular flows. Since the initial condition that $b / h-1$ at the slot was fixed in the analysis, it was expected that there might be some error in the intercept of the growth curve. Each experiment had associated with it a unique virtual origin which determined the intercept of its growth curve. The initial condition was chosen to be a general case, although an experimental value may be used instead. The initial condition also reflected the fact that the analysis assumed self-similar flow from the slot.

Curvature had less of an effect on the maximum velocity decay than it did on the jet growth (figs. 5.5 and 5.6). The decay rate was plotted as $\mathrm{U}_{\mathrm{j}}{ }^{2} / \mathrm{U}_{\mathrm{m}}{ }^{2}$, since the straight wall jet maximum velocity decayed as $x^{-1 / 2}$. This displayed the decay curve as linear for the straight wall. An increase in slope represented an increase in the jet decay rate. The integral computation, for $C_{f}=0$, agreed well with 
previous data. In the annular case, there was little difference in decay rates as the curvature was increased, and this was reflected by the data (fig. 5.5). Fig. 5.6 shows a slightly increased decay rate caused by the streamwise curvature for the two-dimensional wall jet. Again, the virtual origin, which varied from one experiment to another, would cause the intercept of each curve to change. The initial condition for the computation was chosen to be a general case. Although the jet maximum velocity was equal to the jet exit velocity at the slot, there was a potential flow core for several slot heights downstream in an actual flow. In the potential core, the jet velocity did not decay until the end, where the jet became fully developed. This caused a flat section of the curve in the experimental results which was not reflected in the calculation (fig. 4.2). The calculation also assumed self-similar flow from the slot exit. The effect of this error would only be to offset the decay curve upward, and should not affect the slope.

\subsection{Shear Stress Calculation}

Substituting (5.4) and (5.9) into (5.8) yielded the final equation for the shear stress:

$$
\begin{aligned}
& c_{1} \int_{\xi}^{\infty} f^{2} d \xi+c_{2} \int_{\xi}^{\infty} \xi f^{2} d \xi+c_{3} \frac{1}{2} f \int_{0}^{\xi} f d \xi \\
& +C_{4} f \int_{0}^{\xi} \xi f d \xi+c_{5} \xi \int_{\xi}^{\infty} f^{2} d \xi=-\frac{r}{r_{0}} \frac{r}{\rho U_{m}^{2}}
\end{aligned}
$$

where

$$
\begin{aligned}
C_{1} & =\frac{b}{r_{0}} \frac{d r_{0}}{d x}+\frac{1}{U_{m}^{2}} \frac{d\left(b U_{m}^{2}\right)}{d x} \\
C_{2}= & \left(\frac{1}{U_{m}^{2}} \frac{d\left(b U_{m}^{2}\right)}{d x}+\frac{d b}{d x}\right)\left(\frac{b}{r_{0}} \cos \phi-\frac{b}{R}\right) \\
& -\frac{b}{r_{0}} b \sin \phi \frac{d \phi}{d x}+\frac{b^{2}}{R^{2}} \frac{d R}{d x}
\end{aligned}
$$




$$
\begin{aligned}
c_{3}= & \left(\frac{1}{U_{m}^{2}} \frac{d\left(b_{m}^{2}\right)}{d x}+\frac{d b}{d x}\right)+2 \frac{b}{r_{0}} \frac{d r_{0}}{d x} \\
C_{4}= & \frac{b}{r_{0}} \cos \phi\left(\frac{1}{2} \frac{1}{U_{m}^{2}} \frac{d\left(b U_{m}^{2}\right)}{d x}+\frac{3}{2} \frac{d b}{d x}\right) \\
& -\frac{b}{r_{0}} \sin \phi \frac{d \phi}{d x} b \\
C_{5}= & \frac{b}{R} \frac{1}{U_{m}^{2}} \frac{d\left(b U_{m}^{2}\right)}{d x}-\frac{b^{2}}{R^{2}} \frac{d R}{d x}
\end{aligned}
$$

for a curved body of revolution.

The corresponding shear stress equations for special cases were:

2-dimensional plane wall jet:

$$
\begin{aligned}
& c_{1}=\frac{1}{U_{m}^{2}} \frac{d\left(b U_{m}^{2}\right)}{d x} \\
& C_{3}=\frac{1}{U_{m}^{2}} \frac{d\left(b U_{m}^{2}\right)}{d x}+\frac{d b}{d x} \\
& c_{2}=c_{4}=c_{5}=0
\end{aligned}
$$

2-dimensional curved wall jet:

$$
\begin{aligned}
& c_{1}=\frac{1}{U_{m}^{2}} \frac{d\left(b_{m}^{2}\right)}{d x} \\
& c_{2}=\frac{b^{2}}{R^{2}} \frac{d R}{d x} \\
& c_{3}=\frac{1}{U_{m}^{2}} \frac{d\left(b U_{m}^{2}\right)}{d x}+\frac{d b}{d x} \\
& c_{4}=0 \\
& c_{5}=-\frac{b^{2}}{R^{2}} \frac{d R}{d x}
\end{aligned}
$$

axisymmetric plane wall jet:

$$
\begin{aligned}
& C_{1}=\frac{1}{U_{m}{ }^{2}} \frac{d\left(b U_{m}{ }^{2}\right)}{d x} \\
& C_{2}=\left(\frac{1}{U_{m}^{2}} \frac{d\left(b U_{m}^{2}\right)}{d x}+\frac{d b}{d x}\right) \frac{b}{r_{0}}
\end{aligned}
$$

45 


$$
\begin{aligned}
& C_{3}=\frac{1}{U_{m}^{2}} \frac{d\left(b_{m}^{2}\right)}{d x}+\frac{d b}{d x} \\
& C_{4}=\frac{b}{r_{0}}\left(\frac{1}{2} \frac{1}{U_{m}^{2}} \frac{d\left(b U_{m}^{2}\right)}{d x}+\frac{3}{2} \frac{d b}{d x}\right) \\
& C_{5}=0
\end{aligned}
$$

For the two-dimensional equations, the right hand side of the shear stress equation (5.21) was written - $(1+\xi b / R)+/ \rho \mathrm{U}_{\mathrm{m}}{ }^{2}$.

The computational procedure for the integral momentum analysis was as follows. First, the geometry of the model was specified by inputting the streamwise radius of curvature and the annular radius at the slot (both normalized by the slot height). Next, the step size in the downstream direction was specified. At each downstream step location, eqs. (5.14) and (5.15) were solved for $d b / d x$ and $1 / U_{m}^{2} d\left(b U_{m}^{2}\right) / d x$. The quantities $b$ and $U_{m}^{2}$ were computed at each location using these derivatives. Equations (5.14) and (5.15) were updated with the new $b(x)$, and the shear stress profile was computed using the current mean flow data and equation (5.21). The equations were then marched to the next downstream location. The computer programs used to compute both axisymmetric and two-dimensional flow are listed in the appendix.

Figure 5.7 shows the computed shear stress profile for the straight annular wall jet at $\mathrm{x} / \mathrm{h}=106$. The shear stress was zero at the origin, since the skin friction was assumed to be zero. To check for similarity, the peak shear stress was plotted against downstream location in figs. 5.8 and 5.9 For both the annular and two-dimensional straight wall jets, the shear stress was self-similar. As streamwise curvature was introduced, the peaks grew downstream, at a rate which increased as $h / R$ increased. The computed peak shear stress was about 708 higher than the measured shear stress in the straight wall flow.

\subsection{Effects of Skin Friction Coefficient}

The initial version of the computation was made assuming the skin friction coefficient was equal to zero. The overprediction of peak shear stress led to an investigation of the effects of skin friction on the computational results. Since $C_{f}$ is a difficult quantity to 
measure, that measurement was not included in this experiment. The skin friction could either be estimated from previous experiments or computed from the shear stress equation (5.21) evaluated at the wall, using experimentally determined values of $d b / d x$ and $1 / U_{m}^{2} d\left(b U_{m}^{2}\right) / d x$. At $\xi=0$, equation $(5.21)$ is

$$
C_{1} \int_{0}^{\infty} f^{2} d \xi+C_{2} \int_{0}^{\infty} \xi f^{2} d \xi=-\frac{1}{2} C_{f}
$$

for a straight annular wall jet. Using experimental values for $d b / d x$ and $1 / \mathrm{U}_{\mathrm{m}}^{2} \mathrm{~d}\left(\mathrm{bU}_{\mathrm{m}}{ }^{2}\right) / \mathrm{dx}$ gave the relation

$$
\mathrm{c}_{\mathrm{f}}=.011-.056 \mathrm{~b} / \mathrm{ro}
$$

which meant that $C_{f}$ was highly dependent upon the transverse curvature $b / r_{o}$. This method of estimating $C_{f}$ was prone to large error, since the coefficients in equation (5.21) were highly dependent on the measurement accuracy of the growth and decay rates in the jet. Small errors in the measured $b(x)$ or $U_{m}(x)$ would result in large inaccuracies in $C_{f}$. For instance, since

$$
b(x) / h-A\left(x / h-x_{0} / h\right)
$$

and

$$
\mathrm{U}_{\mathrm{m}} / \mathrm{U}_{\mathrm{j}}=\mathrm{B}\left(\mathrm{x} / \mathrm{h}-\mathrm{x}_{\mathrm{o}} / \mathrm{h}\right)^{-\mathrm{k}}
$$

where $A, B$, and $k$ were constants, and assuming the virtual origin $x_{0}$ is the same for both $b$ and $U_{m}$, then

$$
\frac{1}{U_{m}{ }^{2}} \frac{d\left(b U_{m}{ }^{2}\right)}{d x}-\frac{U_{j}{ }^{2}}{U_{m}^{2}} \frac{d\left(b / h U_{m}{ }^{2} / U_{f}{ }^{2}\right)}{d(x / h)}=A(1-2 k) .
$$

Since $k \propto .5$, the term $1 / \mathrm{U}_{\mathrm{m}}{ }^{2} \mathrm{~d}\left(\mathrm{bU}_{\mathrm{m}}{ }^{2}\right) / \mathrm{dx}$ depended on the difference between two nearly equal numbers. A 58 error in the determination of $k$ would lead to a 558 error in the two-dimensional $\mathrm{C}_{f}$.

The alternative method for determining the skin friction coefficient 
was to use an empirical relation between the local jet Reynolds number and $C_{f}$, compiled from previous researchers. ${ }^{4}$ A composite plot of $C_{f}$ vs. Re shows that for the present experiment, $C_{f}$ would be approximately .007 for the straight wall case. It was decided to assume $C_{f}$ was a constant for this calculation. That value was representative of real flows, since previous researchers reported values of $c_{f}$ between .002 and .01 . The value of skin friction coefficient for straight wall jets (based on $U_{m}(x)$ ) is generally higher than the corresponding $C_{f}$ for a flat plate boundary layer in zero pressure gradient (based on $U_{\infty}$ ). In a turbulent boundary layer, $C_{f}$ varies as $\left(U_{\infty} \delta / \nu\right)^{-1 / 4}$. Since $\delta$ varies as $x^{4 / 5}$ and $U_{\infty}$ is constant, $C_{f}$ will vary as $x^{-12} .9$. Correspondingly, in a wall jet, $C_{f}$ varies as $\left(U_{m} y_{m} / \nu\right)^{-.182} .^{4}$ Since the product $U_{m} y_{m}$ varies as $x^{1 / 2}, C_{f}$ will vary as $x^{-.09}$, and will decrease slower downstream than the skin friction coefficient in the boundary layer.

The shear stress equation (5.21) was invariant with skin friction, as it was derived. Since the term containing $C_{f}$ is negligible in (5.15), only (5.14) needed to be modified to include skin friction. The corrected equation ( 5.14 ) had the term $-1 / 2 \quad C_{f} / A_{n}$ added to the right hand side.

Figures 5.10 and 5.11 show the effect of skin friction coefficient on the mean flow. Increasing the skin friction decreased the jet growth and increased the velocity decay in the flow. The effect was larger on the growth rate of the half-width. The shear stress profiles were also computed assuming various values of $C_{f}$. Figure 5.12 shows the decrease in peak shear stress as the skin friction increased. The inclusion of skin friction also allowed for a negative shear stress region near the wall.

In a further attempt to increase the accuracy of the integral method, the assumed velocity profiles used in the integrals in equation (5.21) were replaced by the experimental velocity profiles, which were integrated numerically. This correction was made because of the slight mismatch between the assumed and experimental velocity profiles (fig. 5.2). When the velocity profile was integrated from the wall to the point of peak shear stress, the mismatch would cause the greatest 
error in the integral. A comparison of shear stress profiles using both the assumed and experimental velocity profiles are shown in fig. 5.13 for the straight wall jet. The difference in peak values was significant. Since the velocity profiles for the highly curved wall jet was slightly fuller than the straight jet (fig. 4.7), those velocity profiles matched the assumed profile, and the correction did not have a large effect.

The corrections were applied to the shear stress computation, and it was found that a $\mathrm{C}_{\mathrm{f}}-007$ still overpredicted the peak value. Further investigation found that a value of $C_{f} .013$ provided the best match to the experimental results. The corrected peak shear stress is shown in fig. 5.14 for the straight annular model. Experimental results are also shown.

For the curved jets, the skin friction has been found to vary with streamwise curvature, 21 by the relationship

$$
c_{f}=c_{f_{0}}(1+2 b / R)
$$

where $C_{f o}$ was the value for a straight wall. Choosing the value of $C_{f o}$ which provided a match at the furthest upstream point produced the curves shown in figs. 5.15 and 5.16 for the mildly and highly curved jets. In fig. 5.15, the use of the experimental velocity profiles reproduced the drop in shear stress at $x / h-180$ seen in the data. Although the skin friction coefficient increased with downstream location, the agreement between theory and experiment deteriorated in the highly curved wall jet. Apparently, the analysis cannot accurately compute the highly curved flows downstream, possibly because the streamwise derivatives were no longer negligible as was assumed in the thin shear layer approximation.

Figure 5.17 compares computed and measured shear stress profiles in the straight annular jet. The corrections produced a lateral offset in the profile, probably caused by the lack of velocity measurements close to the wall. Also, the effect of high $C_{f}$ was to worsen the mean flow agreement between analysis and experiment, in spite of the improved peak shear stress agreement. For the general case, the best 
mean flow agreement was achieved for zero skin friction. Added empiricism would probably be necessary to provide a better match when skin friction was included, and improved mean flow agreement might reduce the lateral offset in the shear stress profiles.

To summarize the results from the analysis, the integral calculation was able to predict the same effects due to curvature that were shown experimentally. The growth rate of the jet half-width and the normalized shear stress were seen to increase with curvature in both the annular and two-dimensional calculations. Increasing the skin friction coefficient slightly decreased the growth rate and the peak shear stress.

The mean flow results agreed very well with experiment, but corrections to the skin friction coefficient and the assumed velocity profile were necessary to achieve agreement of the shear stress profiles. The data indicated that the skin friction coefficient may vary with both the streamwise and transverse curvature parameters. 


\section{DISCUSSION}

The primary objective of this research was to investigate experimentally the turbulence properties of a highly curved wall jet. Since previous researchers have had difficulty achieving two-dimensional flow, a secondary objective was to devise and validate an experimental approach which would avoid many of the problems associated with three-dimensional effects.

In the following sections, the experimental approach taken will be evaluated and its advantages and disadvantages discussed. Finally, the experimental approach and the accompanying analysis will be used to discuss the effects of high streamwise curvature on wall jets.

\subsection{Evaluation of Experiment}

One of the objectives of this experiment was to produce a wall jet flow which avoided the problems with three-dimensionality faced by previous researchers. These problems include vorticity created at the slot ends and difficult machining. An annular wall jet model was used to approximate a two-dimensional flow with no end effects. This model was very successful in producing a uniform flow along the span. Easier machining and an improved contraction geometry were other advantages to this model.

For fluctuating velocity measurements, two instrumentation systems were used in this work. The majority of the measurements were taken using cross-wire anemometry. With the use of digital sampling and data reduction, cross-wires can provide accurate measurements of two velocity components. Some disadvantages of cross-wires are the loss of accuracy in the outer shear layer, and the large size of the probe.

Cross-wire measurements in the outer jet region lose accuracy if the mean flow vector is no longer aligned with the probe axis. Since entrainment is high in this region, the normal velocity component may become significant compared with the streamwise velocity. An examination of both the cross-wire and the LDV measurements indicated that the normal velocity component was less than 108 of the streamwise 
velocity up to $\mathrm{y} / \mathrm{b}=2$. Wilson ${ }^{18}$ showed that the mean flow vector was less than $25^{\circ}$ from the wall for both straight and curved wall jets at $\mathrm{y} / \mathrm{b}=2$. These flow angles were within the measurement range of a cross-wire probe. However, an additional error in the outer shear layer was caused when the jet velocity decreased below $4 \mathrm{~m} / \mathrm{s}$, since this was the lower limit of the hot wire calibration. For the straight wall jet at $x / h=60.6$, the mean streamwise velocity had decreased to 4 $\mathrm{m} / \mathrm{s}$ at $\mathrm{y} / \mathrm{b}=1.9$. Further downstream at $\mathrm{x} / \mathrm{h}=128.7$, this limit was reached at $y / b=1.64$. For the highly curved wall jet at $x / h=57$, the mean flow had decreased to $4 \mathrm{~m} / \mathrm{s}$ at $y / b=1.68$. Any measurements outside of this range would be subjected to calibration errors.

For this experiment, the spatial resolution of the probe was mainly a problem in the upstream region of the straight wall jet. With the exception of near-wall measurements, the spatial resolution of the cross-wires was adequate in the curved wall flows, due to the high growth rates in the jets. The cross-wire measurements were believed to be the most accurate in this experiment.

The measurements taken with the IDV system suffered from a high noise level, most likely caused by beam drift due to vibration, in addition to a measuring bias away from detecting entrained particles of fluid. The effect of this bias was to measure velocities which are too high, since the entrained flow was slower than the flow that originated from the slot. The scatter in the mean velocity measurements taken with the LDV showed this effect (fig. 4.4). The normalization of the velocity profile determined that $U / \mathrm{J}_{\mathrm{m}}-1$ at $\mathrm{y}=\mathrm{y}_{\mathrm{m}}$ and $\mathrm{U} / \mathrm{J}_{\mathrm{m}}=1 / 2$ at $\mathrm{y}=\mathrm{b}$. However, in the region $y_{m}<y<b$, the mean velocity was seen to increase downstream, which could be caused by the increased entrainment and velocity bias. The LDV results were mainly used as a comparison for the cross-wires, and allowed mean flow measurements to be taken closer to the wall. However, resolution of the near-wall region was still not adequate with the LDV since the probe volume was approximately four times larger than the microscales in the inner wall region. The feasibility of taking two-component LDV measurements close to a transversely curved wall was demonstrated with the annular model. Measurements in this experiment were taken within $0.4 \mathrm{~mm}$ of the wall, 
and could possibly have been taken closer if the model were painted black to reduce reflections. Future LDV measurements in wind tunnel tests of curved wall jets could be expected to provide useful turbulence data.

Turbulence results were obtained in all three wall jet flows. The important turbulence quantities were the shear stress, which must be modeled in calculation schemes, and the lateral normal stress, since there was widespread disagreement among previous researchers as to the magnitude of this term.

Figures 4.16 and 4.17 show the cross-wire and LDV measurements of the lateral normal stress profiles in the straight annular wall jet, as well as the bandwidth of previous results. A comparison of these figures illustrates the difficulties that exist in measuring this particular quantity. The bias away from measuring entrained flow with the LDV could explain some of the discrepancy in the results. Assuming that the maximum mean velocity measurement was most accurate, since it occurred so close to the wall, the peak turbulence intensities further from the wall should be overpredicted when normalized by $\mathrm{U}_{\mathrm{m}}{ }^{2}$. The bias would have an increasing effect downstream as the amount of entrained fluid in the jet increased. Another explanation of the differences between the LDV and cross-wire results was that the crosswires are somehow in error as well. Work with the cross-wire sampling rates had shown that if there was a time lag between the sampling of the two wires, the quantity $\overline{\mathrm{v}^{\prime 2}}$ would be overpredicted. Since the velocity gradients in the flow were so high near the slot, it would be possible that the spatial gap between the two wires would have the same effect as a temporal lag in the sampling, again causing $\overline{v^{\prime 2}}$ to be overpredicted in the upstream locations.

The shear stress profiles for the straight annular wall jet are shown in figs. 4.20 and 4.21. The profile shapes agreed well with previous two-dimensional results; however, the peak shear stress in the present experiment was in all cases about 258 lower than the peak shear stress obtained by previous researchers. Two possible explanations for this effect were that the transverse curvature in the flow decreased the shear stress in the annular jet, or that the contraction 
flow in this particular model was clean enough to reduce the turbulence levels near the slot. The transverse curvature explanation seemed unlikely for several reasons. A test of the effect of transverse curvature was performed by comparing shear stress profiles measured on the large cylinder radius model and a similar model with a radius a factor of four smaller. The slot height and thus jet half-width were the same with the two models, but the transverse curvature was four times greater on the small cylinder model. Measurements of the shear stress showed no difference in the profiles between the large and small radius models. This would indicate that the transverse curvature would have no effect on the shear stress for a straight wall jet. A vortex stretching hypothesis for diverging flows also does not explain a reduction in shear stress. This hypothesis states that for flows with transverse divergence, vortices with their axes in the transverse direction will get stretched as angular momentum is conserved, and the shear stress would increase. This theory has been supported by a limited number of experiments on transversely diverging flows. The main effect of transverse curvature on the straight wall jet is to cause the outer portions of the jet to diverge, thus having the opposite effect on the shear stress than was observed experimentally.

Measurements of the skin friction coefficient would be a useful addition to the study of annular wall jets. The skin friction was an important quantity in the integral momentum analysis, and it would be instructive to compare the skin friction between annular flows and two-dimensional flows. The measurement of skin friction has been a difficult problem in wall jets. Attempts to obtain the skin friction from either a momentum balance or an extrapolation of the turbulent shear stress near the wall usually lead to erroneous results. ${ }^{4}$ The use of calibrated surface impact probes (Preston tubes) have been used most widely and have given the most reasonable results. However, since the universal law of the wall region is so small in a wall jet, probes of the smallest possible dimensions would need to be used. The very thin jet used in the present experiment would almost exclude the use of impact probes. Preston tube diameters range from $.05 \mathrm{~cm}$ to 0.1 $\mathrm{cm}$, which would encompass the entire inner region of the wall jet in 
the present experiment. Of the previous experiments which used Preston tubes successfully, all have either had slot heights at least 5 times larger than in the present experiment or had a comparable slot height but did not take measurements until over 300 slot heights downstream. ${ }^{4}$

The main disadvantage to annular flow experiments is the introduction of transverse curvature effects. One effect is the transverse convergence or divergence caused by the change in annular radius with downstream distance. A major portion of this work was devoted to finding the limits of validity of the approximation of two-dimensional flow with an annular model.

\subsection{Transverse Curvature Effects}

The integral momentum technique was found to accurately predict the mean flow development of both two-dimensional and annular curved wall jets. The analysis could therefore be a useful tool for finding regions of agreement.

Figures 6.1 and 6.2 compare the half-width growth and maximum velocity decay of both two-dimensional and annular wall jets. Figure 6.1 shows the computed growth of the two straight wall jets, and there was virtually no difference between the two results. This result should be expected, since the ratio of the slot height to cylinder radius was kept small in the annular model. For the mildly curved wall flows, the analysis showed that the annular jet would grow at a similar rate as the two-dimensional jet up until 100 slot heights downstream. After this point, the annular jet would begin to grow much faster than its two-dimensional counterpart. The highly curved wall jet showed the same trend, but the difference between the annular and two-dimensional growth rates remained small over the limited number of slot heights of the analysis.

In fig. 6.2, the results for the maximum velocity decay are shown. Once again, for the straight wall jet, the annular and two-dimensional computations showed no difference in decay rate. The same was true for the mildly curved wall jet until 100 slot heights downstream, where the 
two-dimensional jet decayed faster than the annular jet. In the highly curved jet, the annular and two-dimensional jets were seen to have slightly diverging decay rates, as expected.

From figs. 6.1 and 6.2 , it would seem that an annular straight wall jet could simulate the mean flow development of a two-dimensional straight wall jet to high accuracy. However, annular wall jets with streamwise curvature only agreed with their two-dimensional counterparts in the upstream portions of the flow, presumably because transverse curvature effects on the jet became too large far downstream.

Two parameters which represent transverse curvature have been identified as $b / r_{0}$ and $d r_{0} / d x$. If one of these terms becomes large, transverse curvature will no longer be negligible in the flow. Figure 6.3 plots computed values of these two terms for a range of annular model geometries. The annular radius at the slot was held constant at 316 slot heights, and the streamwise radius of curvature was varied from 20 slot heights to 1000 slot heights. For each geometry, the mean flow quantities $b(x) / h$ and $U_{j}^{2} / N_{m}^{2}(x)$ determined by the two-dimensional and annular calculations were compared. The downstream positions where the mean flow agreement was within 58 and 108 was noted on each curve. Subsequently, these points were joined, thus defining boundaries of agreement between two-dimensional and annular flows. The vertical axis in the figure represents the straight wall case, since $\mathrm{dr} / \mathrm{dx}$ would be equal to zero. This line falls entirely within the good agreement region. Mildly curved geometries achieve high values of $b / r_{0}$ and low values of $d r_{0} / d x$. The opposite is true for highly curved geometries. The computation for the highly curved geometries was halted when the angular distance along the wall reached $90^{\circ}$, and so for extremely high curvatures, this entire flow region would fall into the good agreement portion of the figure. It would be expected that if the transverse radius were increased relative to the slot height, even models with larger streamwise radii of curvature would be able to maintain good mean flow agreement throughout $90^{\circ}$ of arc.

Further examination of the boundaries of agreement revealed that the product of the two terms $b / r_{0}$ and $d r_{0} / d x$ was approximately constant 
along those boundaries. Therefore these regions could be described functionally as:

$$
\begin{aligned}
-b / r_{0} d r_{0} / d x<.016 & \text { good agreement } \\
.016<-b / r_{0} d r_{0} / d x<.032 & \text { moderate agreement } \\
.032<-b / r_{0} d r_{0} / d x &
\end{aligned}
$$

These relations could be used to aid in the design of future annular models, so that regions where two-dimensional flow was satisfactorily approximated could be maximized. For the models used in the present investigation, good agreement occurred over the entire flowfield of the straight wall jet, over the first 90 slot heights of the mildly curved wall jet, and over 26 slot heights of the highly curved wall jet.

The integral momentum analysis was also used to compare the computed shear stresses of the two-dimensional and annular flows. Figures 6.4 through 6.6 show this comparison for the three models. The corrected skin friction coefficient determined in the previous chapter was used in all calculations. The velocity profiles were also corrected for the annular calculations, although not for the two-dimensional calculations. The peak shear stress was plotted in each case. For the straight wall jet (fig. 6.4), the shear stress was self-similar in both computations. There was a slight difference in magnitude, which was entirely accounted for by the velocity profile correction made in one computation and not in the other. If the annular calculation were made without the velocity profile correction, it would overlay the two-dimensional calculation exactly.

A divergence between the two curves was seen in the curved wall jet cases. In both figs. 6.5 and 6.6 , the annular computation predicted a higher growth of the peak shear stress than the two-dimensional computation. However, the experimental results did not confirm this result. Since higher streamwise gradients exist in curved wall jets than in straight jets, the thin shear layer approximations made in the analysis may not be valid in the downstream portions of the flow. 
However, the shear stress results did confirm the regions of agreement found from the mean flow.

The success of the integral momentum technique in validating the annular model concept can be extended to other flows where two-dimensionality is difficult to achieve. One example is the study of transonic turbulent boundary layer separation, which has been studied on an axisymmetric flow model.22 An integral technique might be used over the regions prior to separation for developing a criterion for agreement between axisymmetric and two-dimensional boundary layers. An integral momentum analysis has the advantage of identifying the parameters which are responsible for transverse curvature effects, such as $b / r_{0}$ and $d r_{0} / d x$.

\subsection{Streamwise Curvature Effects}

The streamwise curvature effects on the mean flow have been well documented experimentally. Approximate self-similarity of the mean velocity profile occurred in the curved wall jets as well as in the straight wall jet. The shape of the velocity profiles changed only slightly as streamwise curvature was introduced into the flow. The growth of the jet width changed quite dramatically with the addition of curvature, increasing from a linear growth rate in the straight wall jet to an exponential growth in the curved wall jets. The change in the maximum velocity decay with curvature was not as large as the change in growth rate. The integral analysis was able to predict the increased mixing of a curved wall jet with its surroundings, and the mean flow results from the analysis may be used to validate higher order computational schemes.

The turbulence results in curved wall jets show that although the mean velocities were self-similar, the turbulence quantities were not. Figures 6.7 through 6.9 show the streamwise variation of the peak Reynolds stresses with several values of the curvature parameter $h / R$. These figures compare the present annular results with previous rectangular jet measurements from Refs. 18 and 20 . Wilson 18 provides detailed turbulence measurements in a highly curved wall jet, although 
reviewers suggested that the results suffer from three-dimensional effects. ${ }^{4}$ With $h / R=.0595$, Wilson ${ }^{18}$ reported a very strong growth in the turbulence quantities with downstream distance.

The annular results from the present experiment showed the same trend, but at smaller values of $h / R$. For $h / R=0$, the variation was almost zero, which would be the case for a self-similar turbulent field. The mildly curved flow had a slightly higher growth rate, and the highly curved flow had a proportionately higher growth rate, demonstrating the effect of streamwise curvature on the jet mixing.

The present mildly curved model was patterned after the rectangular model of Alcaraz, 20 which was believed to have good flow uniformity. 4 Alcaraz' results were presented at two slot Reynolds numbers, $1.1 \times 10^{4}$ and $4.0 \times 10^{4}$, which were based on the jet exit velocity and slot height. The lower of the two Reynolds number cases nearly matched the Reynolds number in the present experiment. However, Alcaraz achieved that Reynolds number with a low slot velocity and a large slot height $(8.8 \mathrm{~m} / \mathrm{s}$ and $18 \mathrm{~mm}$ ), compared with the present apparatus ( $90 \mathrm{~m} / \mathrm{s}$ and $1.3 \mathrm{~mm})$. Alcaraz' results revealed large changes in the turbulent stresses due to Reynolds number. For all three turbulent stresses, the growth of the peak stresses increased and the normalized magnitude decreased as the Reynolds number was increased from $1.1 \times 10^{4}$ to $4.0 \mathrm{x}$ $10^{4}\left(U_{j}\right.$ increased from 8.8 to $\left.32 \mathrm{~m} / \mathrm{s}\right)$. Wilson 18 also performed experiments over a wide range of slot Reynolds numbers, but concluded that the wall jet should be Reynolds number invariant at Reynolds numbers above 5000 for the straight jet and 9000 for the very highly curved jet. The conflicting results from these two experiments points out the need for further investigation into the effects of Reynolds number on the flow development. Unfortunately, that investigation could not be carried out in the present work. Increasing the Reynolds number could only be accomplished through an expensive remachining of the annular surfaces to increase the slot height, which would also have the undesirable effect of increasing the relative transverse curvature in the flow. An increase in velocity was not possible, since the air supply was running at maximum output. Higher velocities would also lead to undesirable compressibility effects. Future experimental 
investigations should include the option of varying the slot Reynolds number to investigate the effects on the turbulent stresses.

A comparison of Alcaraz' low Reynolds number results with those from the mildly curved annular model (figs. 6.7 through 6.9) showed similar growth rates of the turbulence quantities; however, the magnitudes of the normalized stresses in Alcaraz' experiment were almost twice as high as the present results. Alcaraz' higher Reynolds number results showed a growth which was much larger than the current results. This comparison served to illustrate the difficulties researchers have faced in creating curved wall jet flows in the laboratory.

Curved wall jets over logarithmic spirals ( $R$ is proportional to $x$ ) have been shown to be self-similar, and the normalized shear stress results collapse to the same curve, since $b / R$ is a constant at all downstream locations. 13 This raises the possibility that turbulence results on circular arc models may also collapse to the same curve if plotted against $b / R$ rather than $x / h$. It was suggested in Ref. 21 that this was true, and with measurements on circular arcs over a wide range of $h / R$, including concave surfaces, the empirical relation was found:

$$
\left(\overline{u^{\prime} v^{\prime}} / U_{m}^{2}\right)_{\max }=0.012(I+5.8 b / R) .
$$

This relationship has been compared to the turbulence results found from other reséarchers investigating circular arc wall jets in fig. 6.10. It was seen that although one or two researchers may find a collapse among data taken on the same experimental rig, there was no agreement between separate experiments. In Alcaraz' experiment, there was no collapse between the two flows at different Reynolds numbers. In the present investigation, the amount of scatter in the curves and the drop in shear stress far downstream in the mildly curved model allowed some leeway in putting a line through the data, and it appeared that a collapse of some sort was possible. Ref. 21 provided the most evidence for a collapse, but their measurements did not agree with anyone else's results. Given the uncertainties present in most of these experiments due to three-dimensionality, it was still inconclusive whether the turbulent shear stress in wall jets over circular 
arcs would collapse to a single curve when plotted against $b / R$, or whether other parameters such as the Reynolds number need to be accounted for as well. 


\section{CONCLUSIONS AND RECOMMENDATIONS}

This experimental investigation had two objectives. The first was to take detailed turbulence measurements in highly curved wall jets in order to study the effects of curvature on the turbulent structure in the flow. In light of the difficulties previous researchers have faced, the second objective was to devise and validate an experimental approach which minimized three-dimensional effects in the flowfield.

An annular wall jet model was chosen in order to achieve a high quality flow that was free of spanwise nonuniformities. The main advantages of annular models were the elimination of end effects and the ability to manufacture a slot and contraction which were uniform and free of internal support structure. Another advantage was the improved optical access to the surface available to a two-component Laser Doppler Velocimetry system. All of these advantages offered an improvement over the difficulties associated with rectangular slot experiments.

The transverse curvature associated with an annular flow was the main disadvantage to these models when trying to simulate two-dimensional flow. An integral momentum analysis was formulated to predict the effects of both streamwise and transverse curvature on wall jets. With the use of the analysis, it was found that straight annular wall jet models can satisfactorily approximate two-dimensional flow. However, if the wall was curved, a criterion existed which would define the regions where the two-dimensional approximation was valid. Two transverse curvature terms have been identified as $b / r_{0}$ and $-d r_{0} / d x$. If the product of these two terms was less than 0.016, there was agreement to within $5 \%$ between the annular and two-dimensional mean flows. If the product was less than 0.032 , the two flows will agree to within 10\%. At any value of the product over 0.032 , there will be poor agreement between annular and two-dimensional flow.

The annular wall jet experiment has been successfully performed with a straight wall, a mildly curved wall, and a highly curved wall. The effects of streamwise curvature on the flowfield, such as the increased 
growth rate and the increase in the peak shear stress, have been demonstrated.

The integral momentum analysis was able to predict the effects of streamwise curvature on the mean flow in both two-dimensional and annular wall jets. The shear stress in the straight wall jet was also predicted, as long as the appropriate skin friction and velocity profiles were used.

Both the annular model concept and the integral momentum technique have been validated and may be used as test cases for higher order computational schemes. Recommendations for future work include using the criterion developed for the regions of agreement between annular and two-dimensional flow to design future annular models.

The measurement of skin friction in annular flows should be performed in order to determine possible transverse curvature effects. A measured value should also be used in the integral momentum analysis and would be necessary for higher order computational schemes as well. Experiments should be performed at various values of the slot Reynolds number, so that Reynolds number invariance may be verified.

The annular geometry approach and the integral momentum technique should be extended to examine curvature effects on wall jet mixing with an external stream. In particular, any future experiments of this type should take advantage of the improved optical access for an LDV system offered by annular models. 
1. Fekete, G. I., "Coanda Flow in a Two-Dimensional Wall Jet on the Outside of a Cylinder", Mech. Eng. Dept. Rep. 63-11, McGill University, 1963.

2. Williams, R. M., and Rogers, E. O., "Design Considerations of Circulation Control Rotors", 28th Annual National Forum of the American Helicopter Society, Washington, D.C., May 1971.

3. Wille, R., and Fernholz, H., "Report on the First European Mechanics Colloquium on the Coanda Effect", Journal of Fluid Mechanics, vol. 23, part 4, p.801, 1965.

4. Launder, B. E. and Rodi, W., "The Turbulent Wall Jet", Prog. Aerospace Sci., Vol. 20, No. 2, Feb. 1977.

5. Newman, B. G., "The Deflexion of Plane Jets by Adjacent Boundaries--Coanda Effect", Boundary Layer and Flow Control, Vol. I, p. 232, Pergamon Press, 1961.

6. Sawyer, R. A., "Two-Dimensional Turbulent Jets with Adjacent Boundaries", Ph.D. Thesis, Cambridge University, 1962.

7. Patel, R. P. and Newman, B. G., "Self-Preserving Two-Dimensional Turbulent Jets and Wall Jets in a Moving Stream", Mech. Eng. Rep. Ae5, McGill University, 1961.

8. Bradshaw, P., "Effects of Streamline Curvature on Turbulent Flow", AGARD-AG-169, August 1973.

9. Schlichting, H., Boundary-Layer Theory, Seventh Edition, McGraw-Hill, 1979.

10. Patankar, S. V. and Spalding, D. B., Heat and Mass Transfer in Boundary Layers, London:Morgan-Grampian, 1967.

11. Launder, B. E. and Rodi, W., "The Turbulent Wall Jet--Measurements and Modeling", Ann. Rev. Fluid Mech., Vol. 15, p. 429, 1983. 
12. Rodi, W. and Scheuerer, G., "Calculation of Curved Shear Layers with Two-Equation Turbulence Models", Phys. Fluids, vol. 26, no. 6, p. 1422, June 1983.

13. Guitton, D. E. and Newman, B. G., "Self-Preserving Turbulent Wall Jets over Convex Surfaces", J. Fluid Mech., vol. 81, part 1, p. 155, 1977.

14. Gartshore, I. and Hawaleshka, 0., "The Design of a Two-Dimensional Blowing Slot and its Application to a Turbulent Wall Jet in Still Air", Tech. Note 64-5, Dept. of Mech. Eng., McGill University, Montrea1, 1964.

15. Englar, R. J. and Williams, R. M., "Test Techniques for HighLift, Two-Dimensional Airfolls with Boundary Layer and Circulation Control for Application to Rotary Wing Aircraft", NSRDC 4645, July 1975.

16. McGahan, W. A., "The Incompressible, Turbulent Wall Jet in an Adverse Pressure Gradient", Rept. No. 82, Gas Turbine Laboratory, Massachusetts Institute of Technology, Sept. 1965.

17. Wilson, D. J. and Goldstein, R. J., "Turbulent Wall Jets with Cylindrical Streamwise Curvature", J. of Fluids Eng., vol. 98, p. 550 , Sept. 1976 .

18. Wilson, D. J., "An Experimental Investigation of the Mean Velocity, Temperature and Turbulence Fields in Plane and Curved Two-Dimensional Wall Jets: Coanda Effect", Ph.D. Thesis, Mech. Eng. Dept., University of Minnesota, 1970.

19. Alcaraz, E., Charnay, G. and Mathieu, J., "Measurements in a Wall Jet over a Convex Surface", The Physics of Fluids, vol. 20, no. 2, Feb. 1977.

20. Alcaraz, E., "Contribution à l'Etude d'un Jet Plan Turbulent Evoluant le long d'une Parol Convexe à Falble Courburen, These d'Etat, Universite Claude Bernard, Lyon, France, 1977.

21. Kobayashi, R. and Fujisawa, N., "Curvature Effects on TwoDimensional Turbulent Wall Jets", Ingenieur-Archiv, vol. 53, p. $409,1983$. 
22. Bachalo, W. D. and Johnson, D. A., "Transonic Turbulent Boundary Layer Separation Generated on an Axisymmetric Flow Model", AIAA Journal, vol. 24, no. 3, p. 437, March 1986.

23. Bearman, P. W., "Corrections for the effect of Ambient Temperature Drift on Hot-Wire Measurements in Incompressible Flow," DISA Information, No. 11, May 1971.

24. Wood, N. J., "The Aerodynamics of Circulation Control Aerofoils," JIAA TR-41, Dept. of Aero/Astro, Stanford Univ., July 1981.

25. Westphal, R. V. and Mehta, R. D., "Crossed Hot-Wire Data Acquisition and Reduction System," NASA Technical Memorandum 85871, NASA Ames Research Center, January 1984.

26. Rodman, L. C., Bell, J. H. and Mehta, R. D., "A 3-Component Laser Doppler Velocimeter Data Acquisition and Reduction System", JIAA TR-63, Dept. of Aero/Astro, Stanford University, August 1985 .

27. Roberts, L., "A Theory for Turbulent Curved Wall Jets", AIAA-870004, AIAA 25th Aerospace Sciences Meeting, Reno, NV, January $12-15,1987$.

28. Cebeci, T. and Bradshaw, P., Momentum Transfer in Boundary Layers, McGraw-Hill, 1977. 


\begin{abstract}
APPENDIX
Two computer programs were written to perform the integral momentum calculation over curved wall jets. The first program, ANNWJ, computes the annular curved wall jet. The second program, TWODWJ, computes the two-dimensional wall jet flowfield. These programs were run on an IBM PC.

The annular wall jet program requires three geometric inputs:

$\Delta(\mathrm{x} / \mathrm{h})=$ spacial marching step normalized by the slot height,

$\mathrm{R} / \mathrm{h}=$ the constant streamwise radius of curvature normalized by the slot height, and

$r_{0}(1) / h$ - the annular radius at the slot normalized by the slot height.

The straight wall case can be produced by the input of a sufficiently large streamwise radius $\left(e . g . R / h=1 \times 10^{6}\right)$.

The spacial marching step $\Delta(\mathrm{x} / \mathrm{h})$ is dependent on the streamwise radius of curvature. The increment should be less than or equal to the total downstream distance divided by 20 steps. The total downstream distance should terminate before an angular distance of $90^{\circ}$ is reached. Thus,

$$
\Delta(\mathrm{x} / \mathrm{h}) \leq(\mathrm{R} / \mathrm{h} * \pi / 2) / 20
$$
\end{abstract}

A smaller $\Delta(\mathrm{x} / \mathrm{h})$ leads to higher computational accuracy.

Various constants are set in the program, which are given below:

$G=K$ in eq. (5.13) - .073 = the planar growth rate $\mathrm{db} / \mathrm{dx}$ of a wall jet $C=C_{n} / A_{n}$ in eq. $(5.12)=.503$ - constant in mean flow equation $\mathrm{AN}=\mathrm{n}$ in eq. (5.9) $=6$ - constant in velocity profile $\operatorname{PAK}=\kappa$ in eq. $(5.9)-.8814$ - constant in velocity profile $\mathrm{XM}=\xi_{\mathrm{m}}$ in eq. $(5.9)-.159-1 /(1+\kappa n)=$ constant in velocity profile.

The skin friction coefficient $C_{f}$ is also an input to the program. It is multiplied by $3 \kappa / 4$ to put it in the form used by eq. (5.14). This 
version of the program assumes a constant skin friction coefficient. An alternative would be to specify that $C_{f}$ is a function of $b / R$.

The angular position along the wall and the annular radius are then computed at each downstream location. The downstream location $x / h$ is determined as well. If the straight wall jet is to be computed, the annular radius should be set equal to a constant in the program.

At each downstream step, the matrix equation which combines eqs. (5.14) and (5.15) is solved. The boundary conditions at the slot are:

$$
\begin{aligned}
& \mathrm{b} / \mathrm{h}=1 \\
& \mathrm{U}_{\mathrm{m}}^{2} / \mathrm{U}_{\mathrm{j}}{ }^{2}=1 \\
& \mathrm{~b} / \mathrm{h} \mathrm{U}_{\mathrm{m}}^{2} / \mathrm{U}_{\mathrm{j}}^{2}=1 .
\end{aligned}
$$

The matrix elements and the vector on the right-hand-side are input using the local mean flow and geometry. A standard matrix solver (SIMQ) is called to compute the vector $\left[\mathrm{db} / \mathrm{dx} \quad 1 / \mathrm{U}_{\mathrm{m}}{ }^{2} \mathrm{~d}\left(\mathrm{bU}_{\mathrm{m}}{ }^{2}\right) / \mathrm{dx}\right]^{\mathrm{T}}$. These derivatives and the current mean flow variables $b / h$ and $U_{m}{ }^{2} / U_{j}{ }^{2}$ are used to compute the mean flow at the next spacial location, using an Euler explicit approximation.

The coefficients $C_{1}$ through $C_{5}$ in the shear stress equation (5.21) are next computed. The shear stress profile is then calculated at the current location. In eq. (5.21), each integral function of $f$ and $\xi$ was found in closed form, where $f=U / J_{m}$ is given by eq. (5.9). These functions are given by A1 through A6 for $\xi \leq \xi_{\mathrm{m}}$ and AINT1 throught AINT6 for $\xi>\xi_{\mathrm{m}}$ and are defined below:

AI and AINT1: $\quad \int_{0}^{\xi} f^{2} d \xi$

A2 and AINT2: $\quad \frac{1}{2} f \int_{0}^{\xi} f d \xi$ 
A3 and AINT3: $\quad \int_{0}^{\xi} \xi f^{2} d \xi$

A4 and AINT4: $\quad \mathrm{f} \int_{0}^{\xi} \xi \mathrm{fd} \xi$

A5 and AINT5: $\int_{\xi}^{\infty} f^{2} \mathrm{~d} \xi$

A6 and AINT6: $\quad \int_{\xi}^{\infty} \xi \mathrm{f}^{2} \mathrm{~d} \xi$

These functions are multiplied by the corresponding coefficient to give the shear stress profile. The program marches to the next downstream location, and the process repeats.

The two-dimensional wall jet program, TWODWJ, is much simpler than ANNWJ since the mean flow variables may be found in closed form. These quantities are given by:

$$
\begin{aligned}
& \frac{d b}{d x}=k\left(1+\frac{n}{\kappa} \frac{b}{R}\right)-C_{f}\left[\frac{3 \kappa}{4}-\frac{n}{2} \frac{b}{R}\right] \\
& \frac{1}{U_{m}^{2}} \frac{d\left(b U_{m}{ }^{2}\right)}{d x}=-\frac{1}{2} C_{f} \frac{(1+\kappa n)}{(1+2 n / 3)}
\end{aligned}
$$

The shear stress is given by:

$$
\frac{\overline{u^{\prime} v^{\prime}}}{U_{m}^{2}}-\frac{C_{1} \text { AINT5 }+C_{3} \text { AINT2 }}{1+b \xi / R}
$$

where

$$
C_{1}=\frac{1}{U_{m}^{2}} \frac{d\left(b U_{m}^{2}\right)}{d x}
$$

71 


$$
C_{3}=\frac{1}{U_{m}^{2}} \frac{d\left(b_{m}^{2}\right)}{d x}+\frac{d b}{d x}
$$

In this version, the skin friction coefficient for curved wall jets was found by multiplying the given straight wall jet value by the quantity $(1+2 b / R)$ (see Sec. 5.5). 


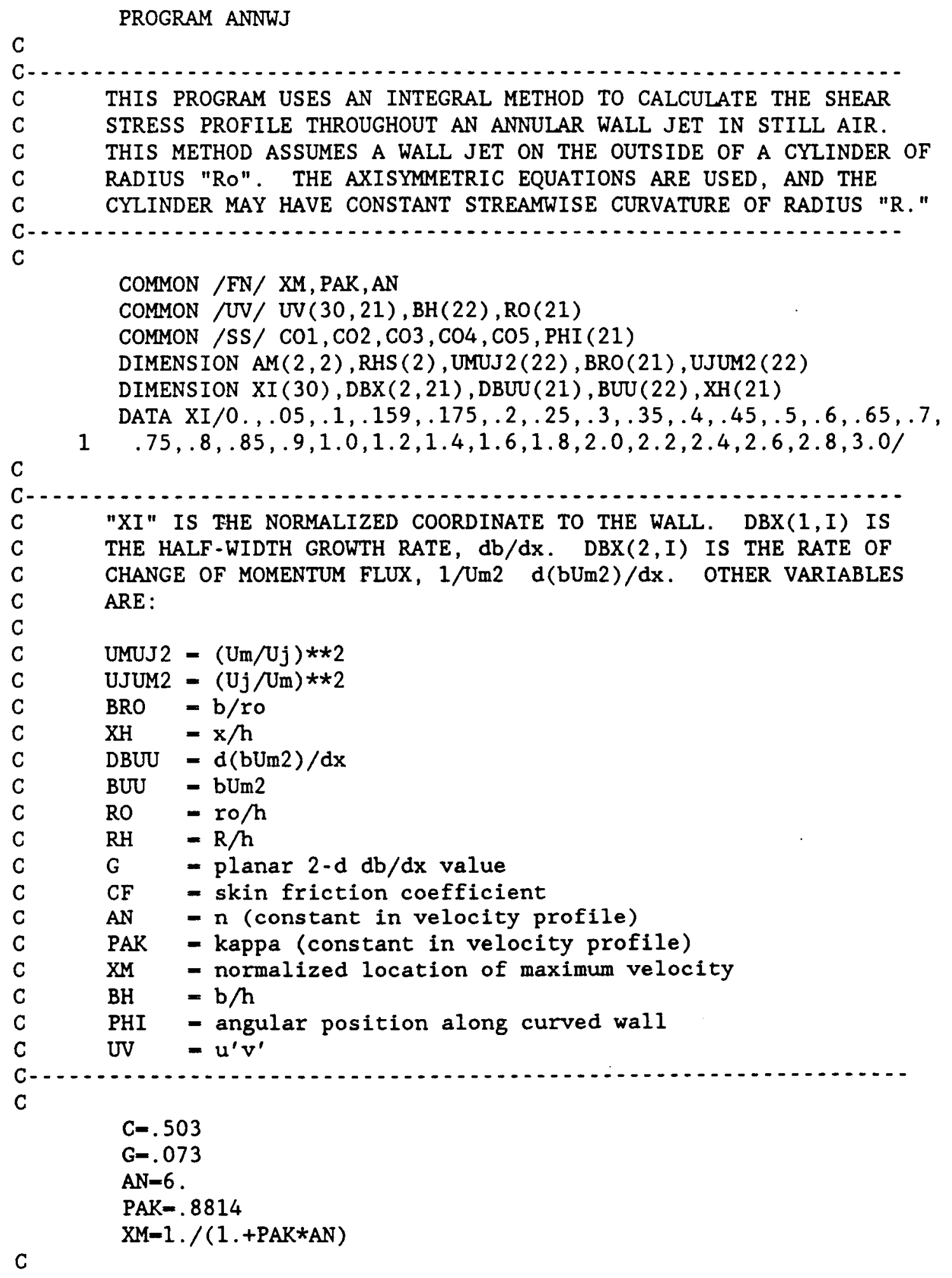




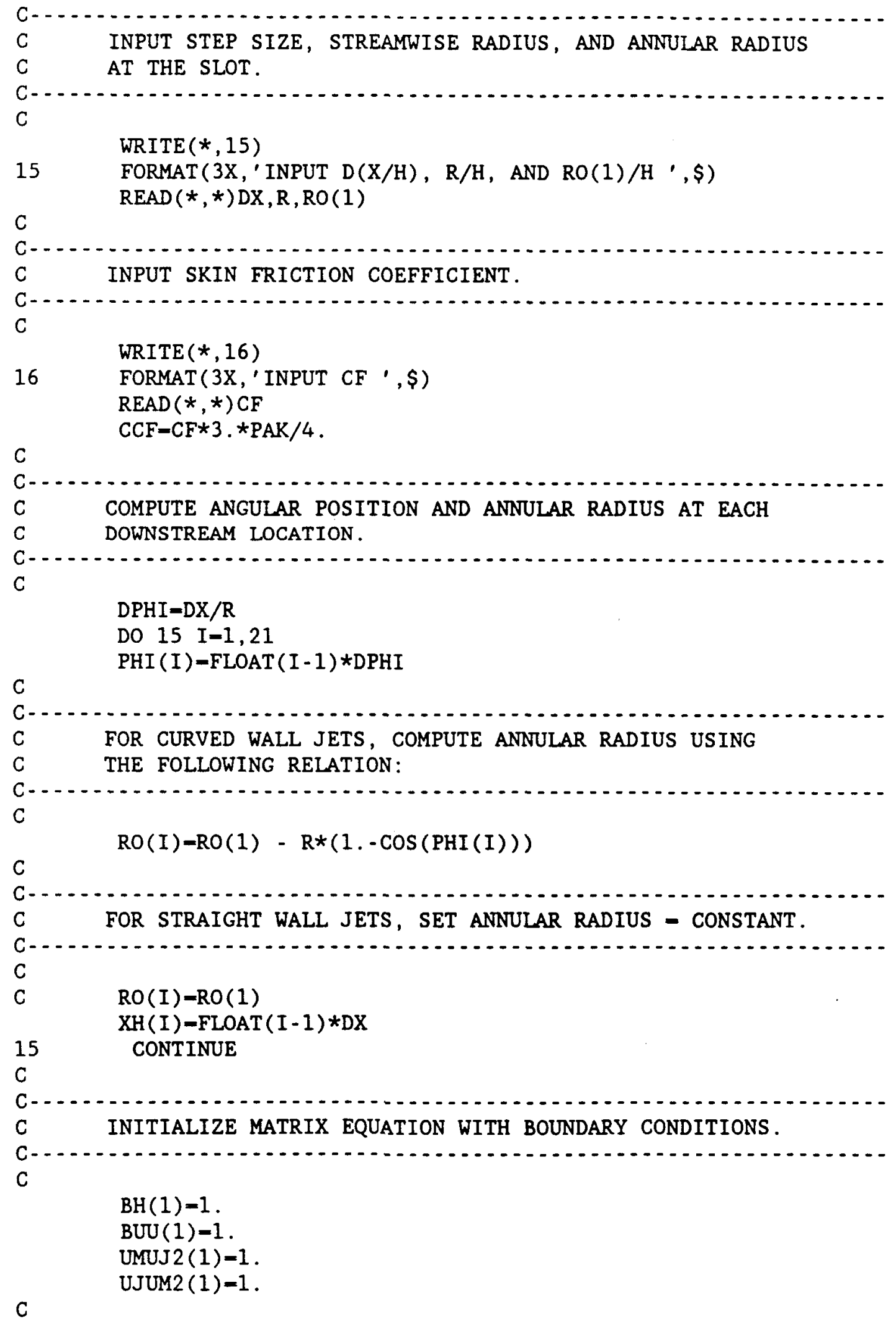




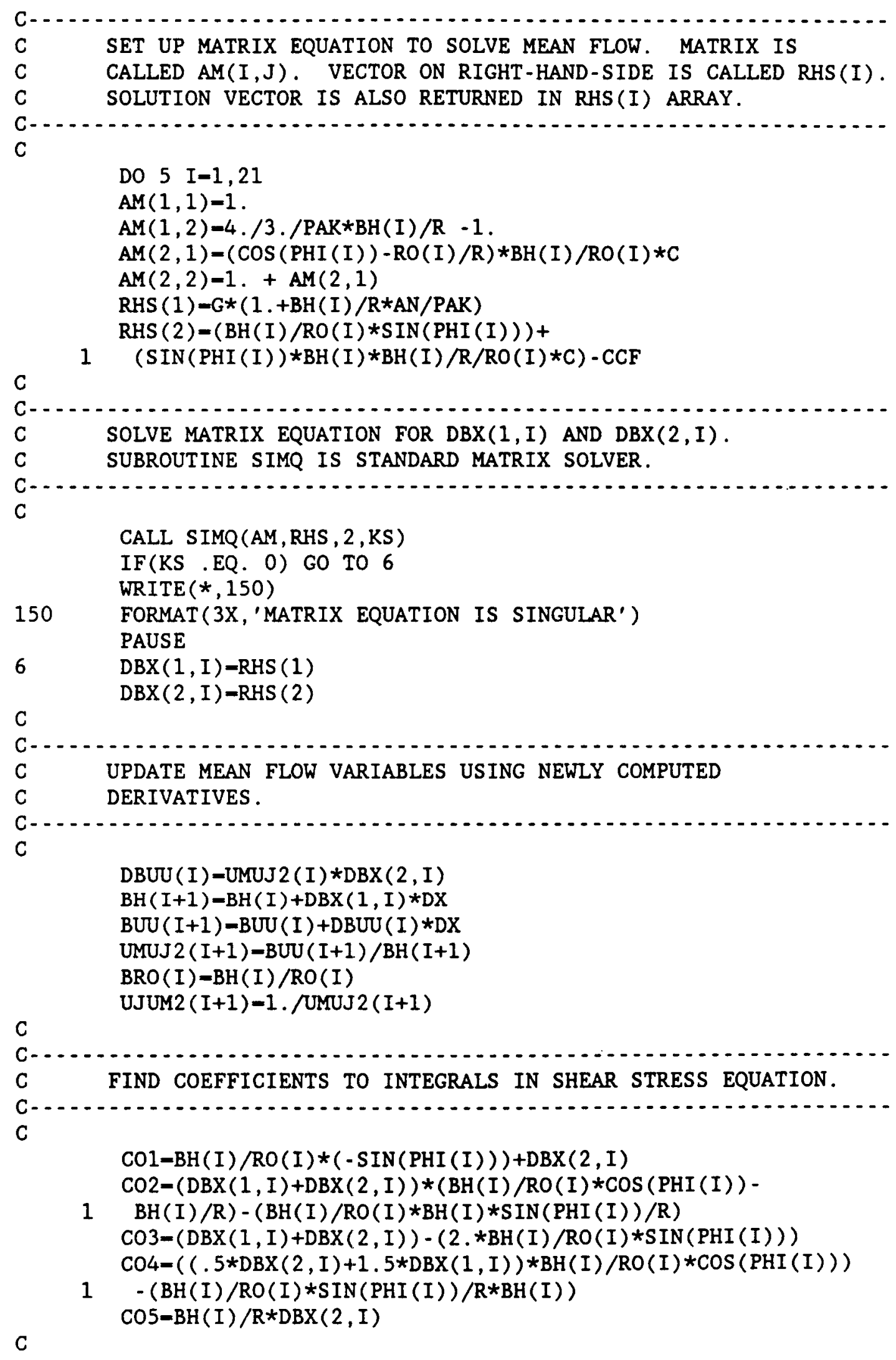




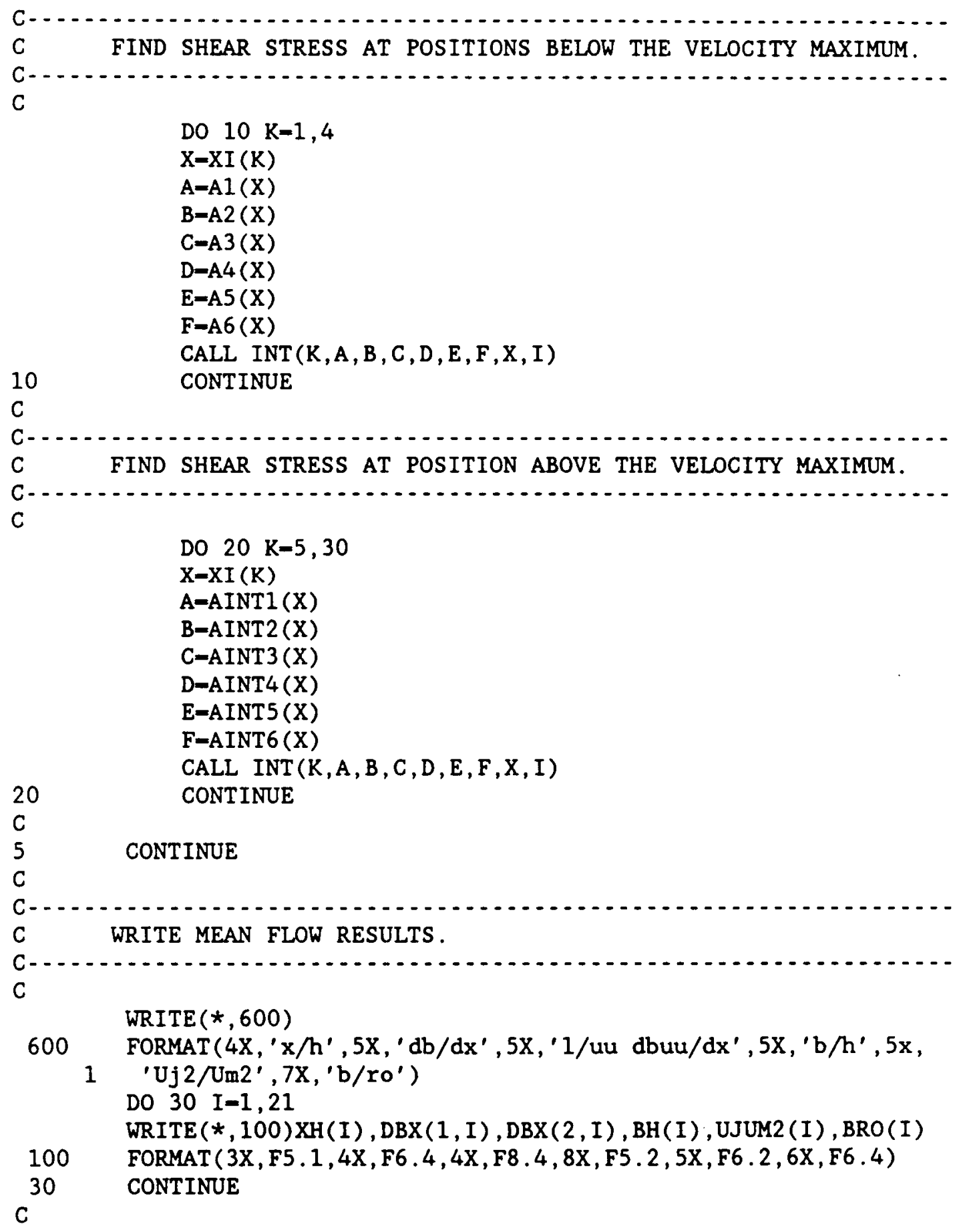




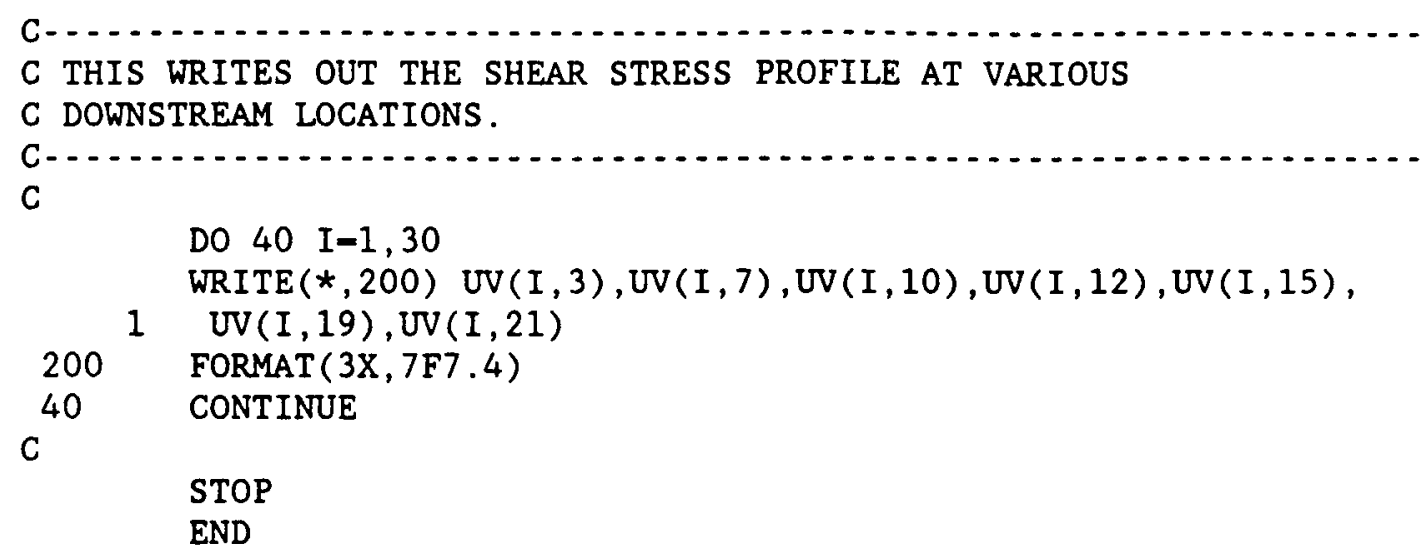

END 


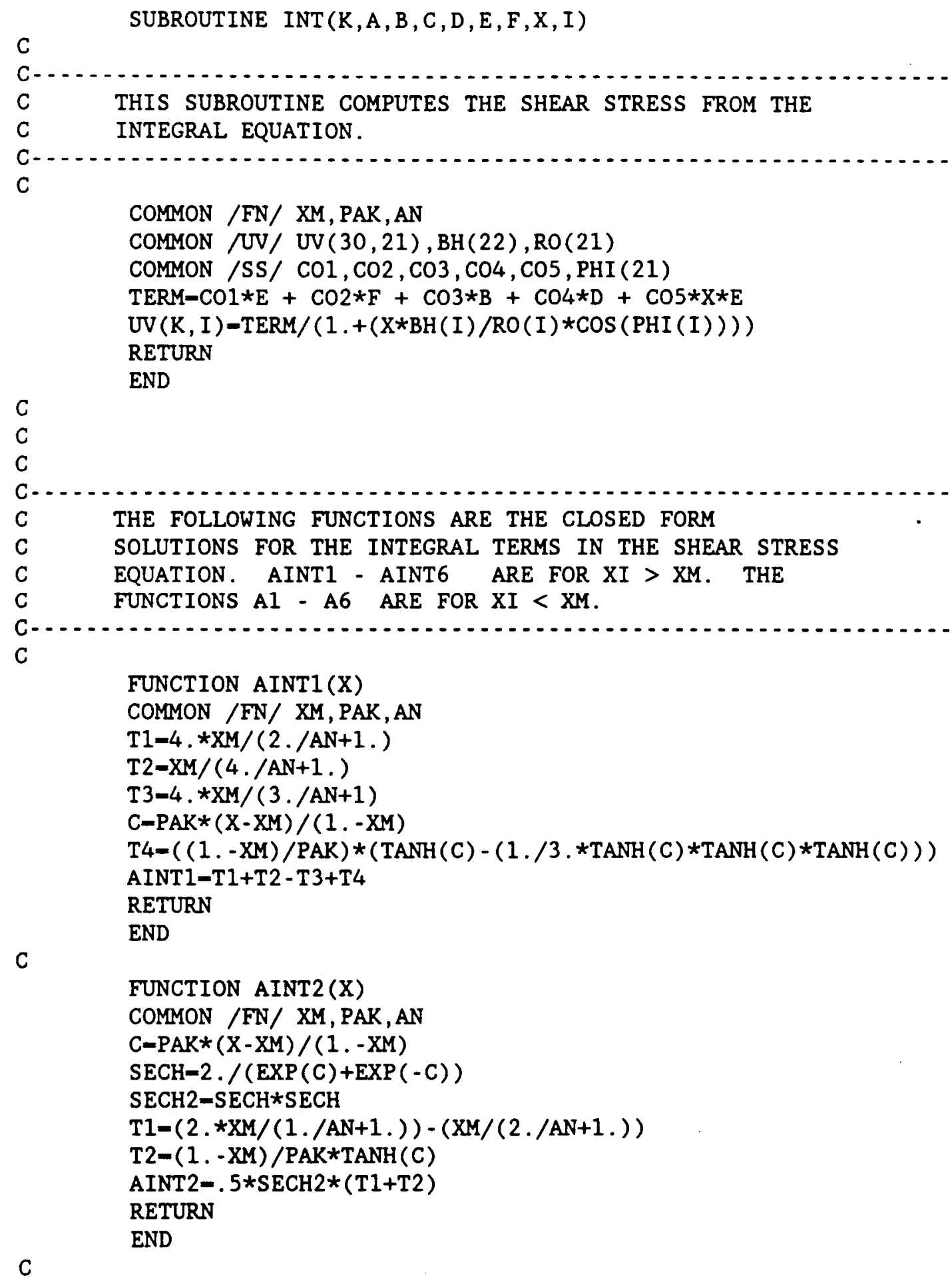

C 


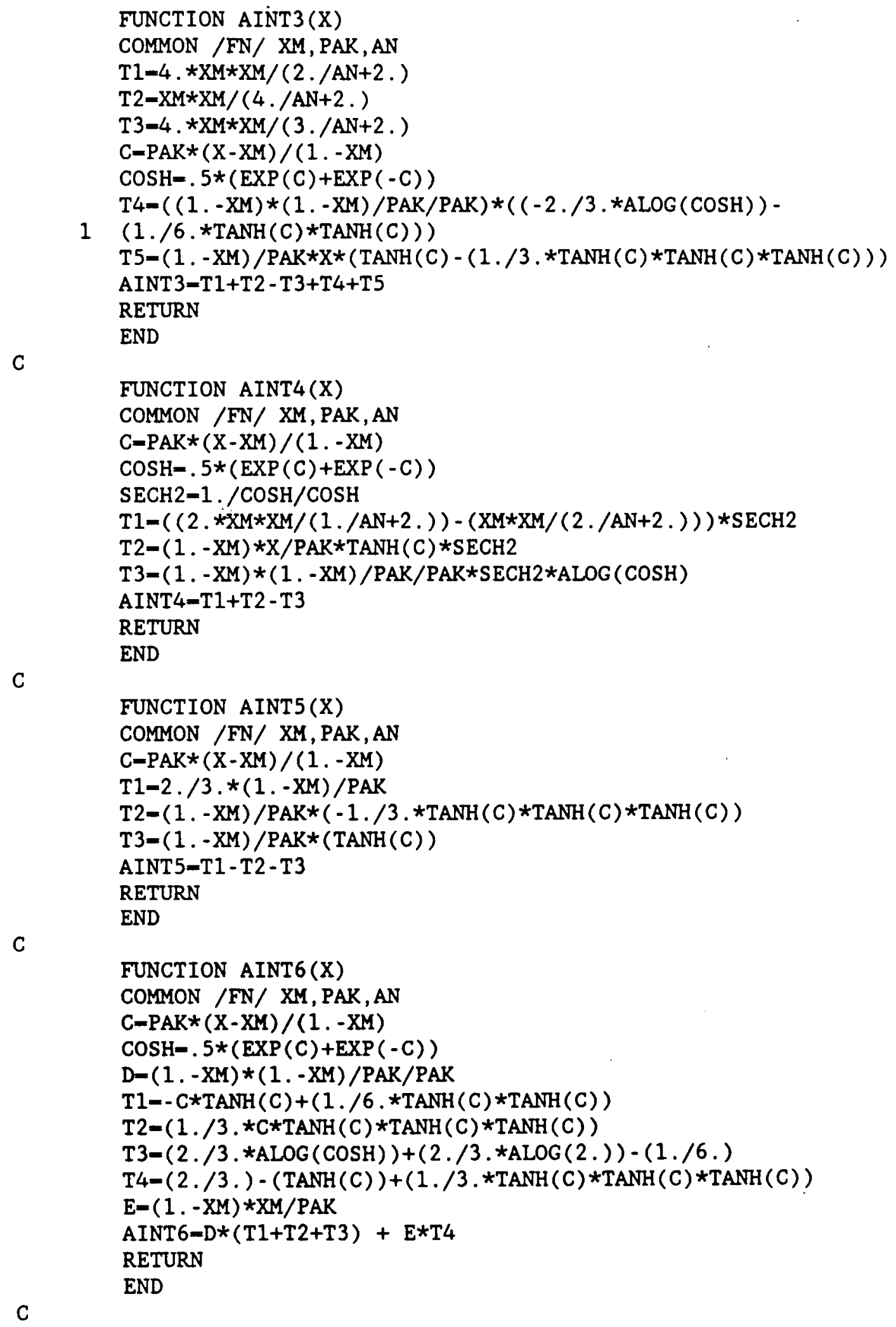


FUNCTION Al(X)

COMMON /FN/ XM, PAK, AN

$\mathrm{V}=\mathrm{X} / \mathrm{XM}$

$A 1=4 . * X M /(2 . / A N+1) * V * *.(2 . / A N+1)+.X M /(4 . / A N+1) * V * *.(4 . / A N+1$.

$1-4 . * X M /(3 . / A N+1) * V * *.(3 . / A N+1$.

RETURN

END

C

FUNCTION A2(X)

COMMON /FN/ XM, PAK, AN

$\mathrm{V}=\mathrm{X} / \mathrm{XM}$

$\mathrm{A} 2=(2 . * \mathrm{XM} /(1 . / \mathrm{AN}+1). * \mathrm{~V} * *(1 . / \mathrm{AN}+1)-\mathrm{XM} /(2 . / \mathrm{AN}+1). * \mathrm{~V} * *(2 . / \mathrm{AN}+1)$.

$1 *(\mathrm{~V} * *(1 . / \mathrm{AN})-.5 * \mathrm{~V} * *(2 . / \mathrm{AN}))$

RETURN

END

C

FUNCTION A3(X)

COMMON /FN/ XM, PAK, AN

$\mathrm{V}=\mathrm{X} / \mathrm{XM}$

$\mathrm{A} 3=\mathrm{XM} * \mathrm{XM} *(4 . /(2 . / \mathrm{AN}+2). * \mathrm{~V} * *(2 . / \mathrm{AN}+2)+.1 . /(4 . / \mathrm{AN}+2). * \mathrm{~V} * *(4 . / \mathrm{AN}+2)$

1. $-4 . /(3 . / \mathrm{AN}+2) * \mathrm{~V} * *(3 . / \mathrm{AN}+2)$.

RETURN

END

C

FUNCTION A4(X)

COMMON /FN/ XM, PAK, AN

$\mathrm{V}=\mathrm{X} / \mathrm{XM}$

$A 4=(2 . * X M * X M /(1 . / A N+2) * V * *.(1 . / A N+2)-.X M * X M /(2 . / A N+2) *$.

$1 \mathrm{~V} * *(2 . / \mathrm{AN}+2)) *.(2 . * \mathrm{~V} * *(1 . / \mathrm{AN})-\mathrm{V} * *(2 . / \mathrm{AN}))$

RETURN

END

C

FUNCTION A5 (X)

COMMON /FN/ XM, PAK, AN

$\mathrm{V}=\mathrm{X} / \mathrm{XM}$

$A 5=(2 . / 3 . *(1 .-X M) / P A K)+(4 . * X M /(2 . / A N+1) *.(1 .-V * *(2 . / A N+1))$.

$1-(4 . * \mathrm{XM} /(3 . / \mathrm{AN}+1) *.(1 .-\mathrm{V} * *(3 . / \mathrm{AN}+1)))+.(\mathrm{XM} /(4 . / \mathrm{AN}+1) *$.

$1 \quad(1 .-V * *(4 . / A N+1))$.

RETURN

END

C

FUNCTION A6(X)

COMMON /FN/ XM, PAK, AN

$\mathrm{V}=\mathrm{X} / \mathrm{XM}$

$\mathrm{T} 1=(1 .-\mathrm{XM}) *(1 .-\mathrm{XM}) / \operatorname{PAK} / \operatorname{PAK} *(2 . / 3 . * A L O G(2)-.1 . / 6$.

$\mathrm{T} 2-2 . / 3 . *(1 .-\mathrm{XM}) / \mathrm{PAK} * \mathrm{XM}$

$\mathrm{T} 3=(4 . * \mathrm{XM} * \mathrm{XM} /(2 . / \mathrm{AN}+2)) *.(1 .-\mathrm{V} * *(2 . / \mathrm{AN}+2)$.

$\mathrm{T} 4=(4 . * \mathrm{XM} * \mathrm{XM} /(3 . / \mathrm{AN}+2)) *.(1 .-\mathrm{V} * *(3 . / \mathrm{AN}+2)$.

$\mathrm{T} 5=(\mathrm{XM} * \mathrm{XM} /(4 . / \mathrm{AN}+2)) *.(1 .-\mathrm{V} * \star(4 . / \mathrm{AN}+2)$.

$\mathrm{A} 6=\mathrm{T} 1+\mathrm{T} 2+\mathrm{T} 3-\mathrm{T} 4+\mathrm{T} 5$

RETURN

END 


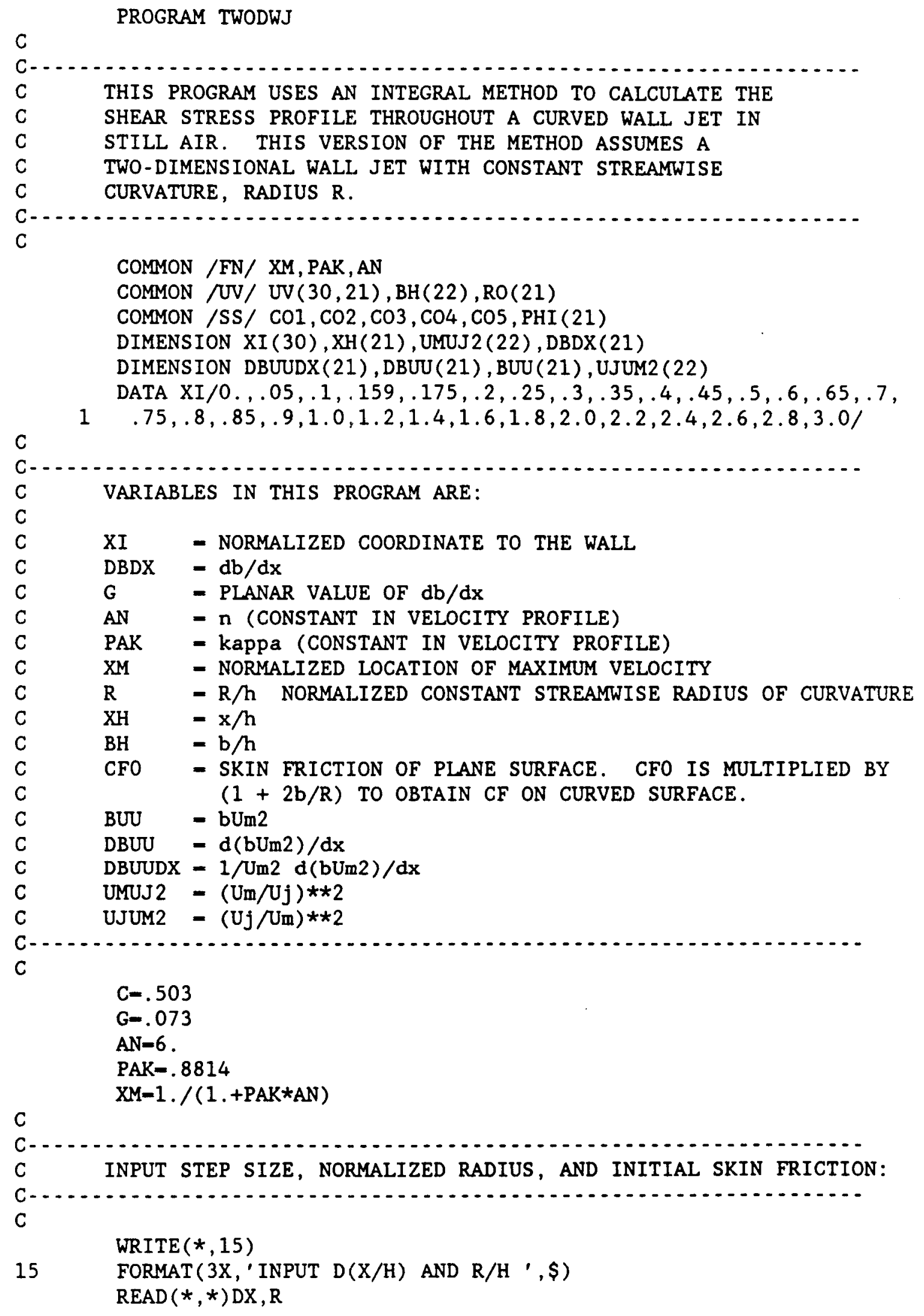




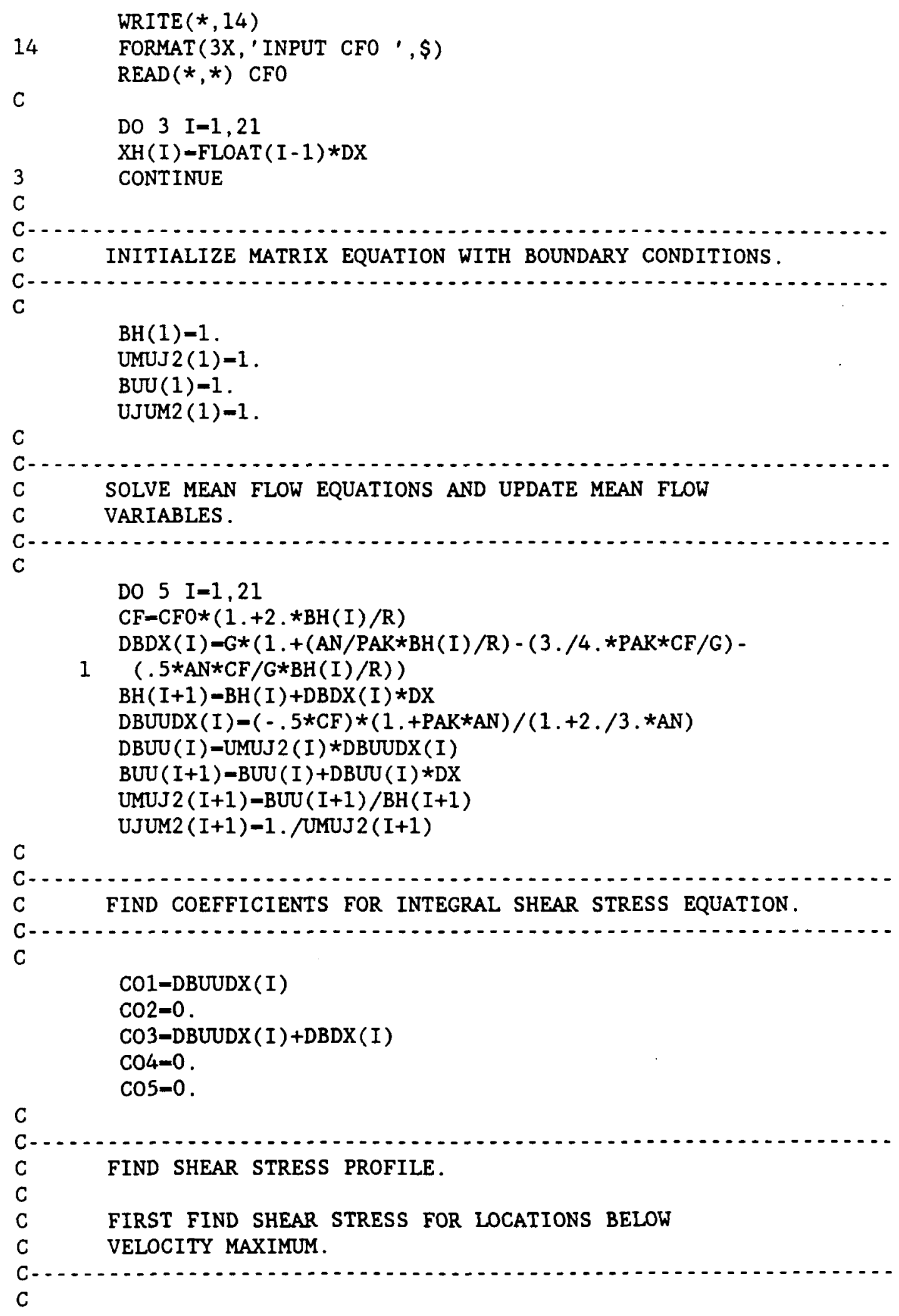




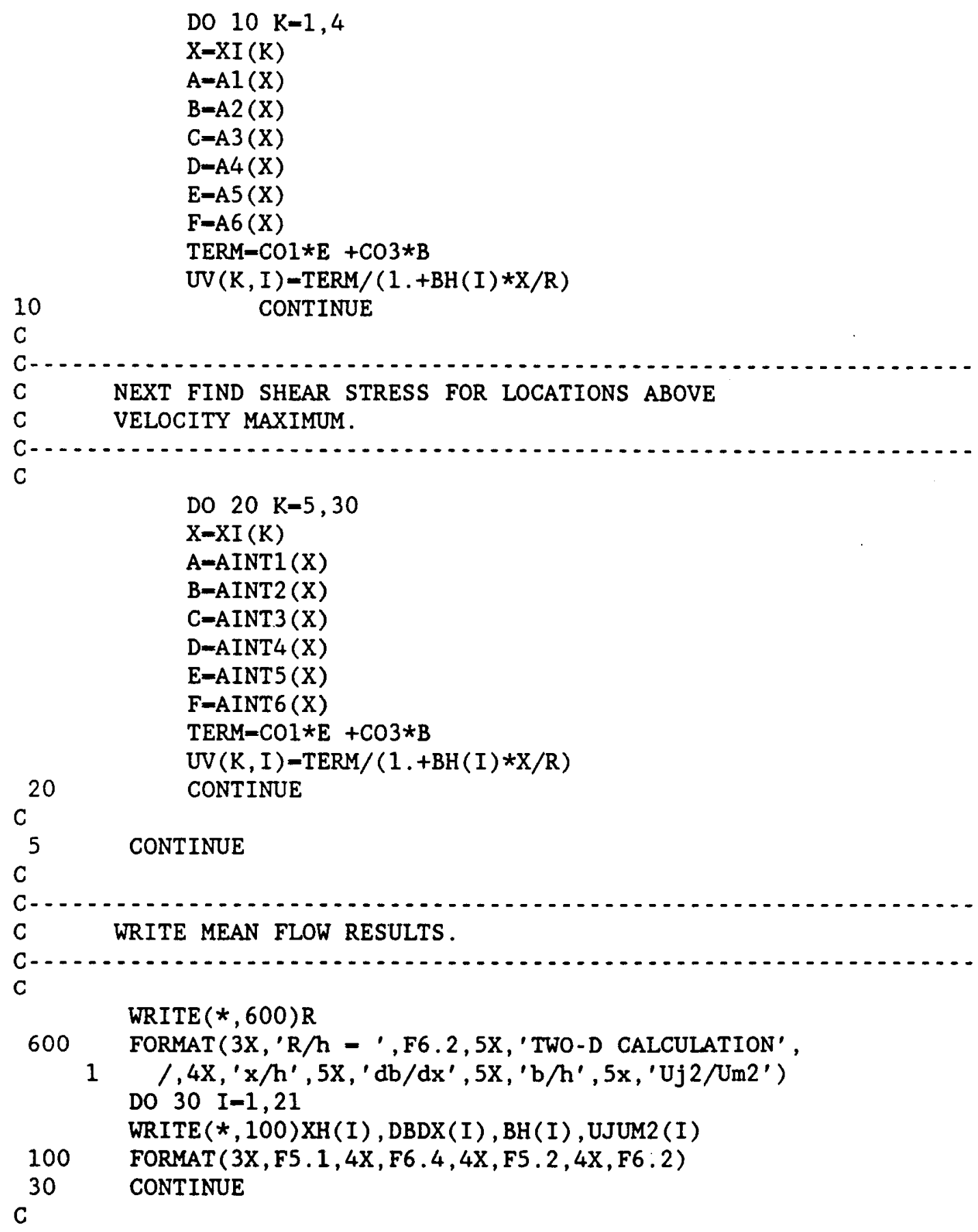




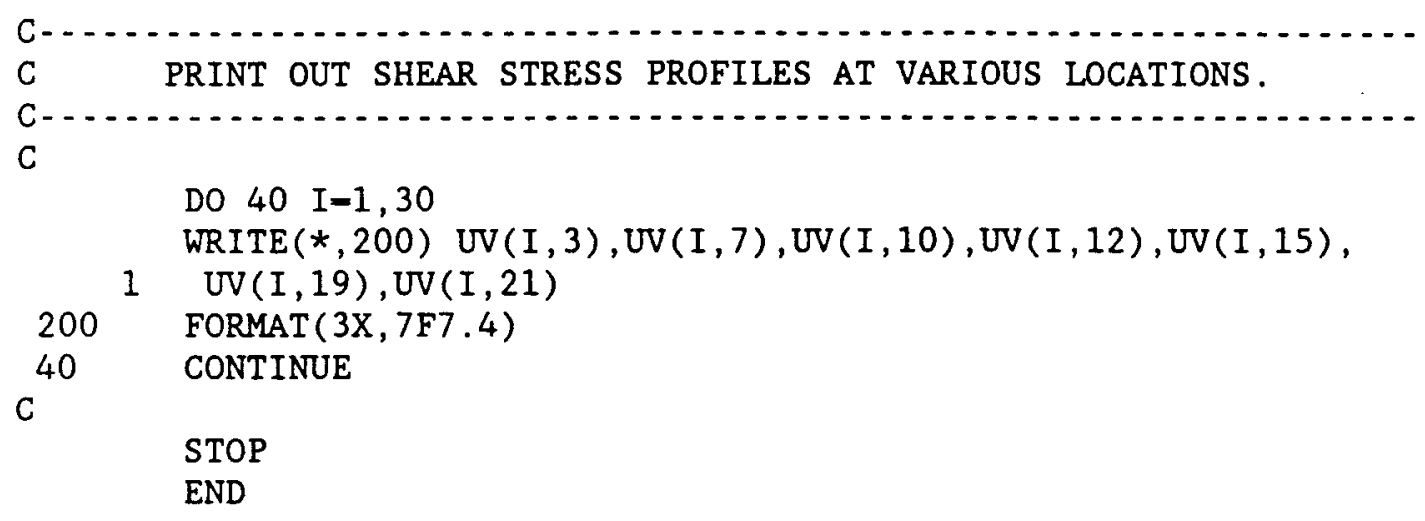




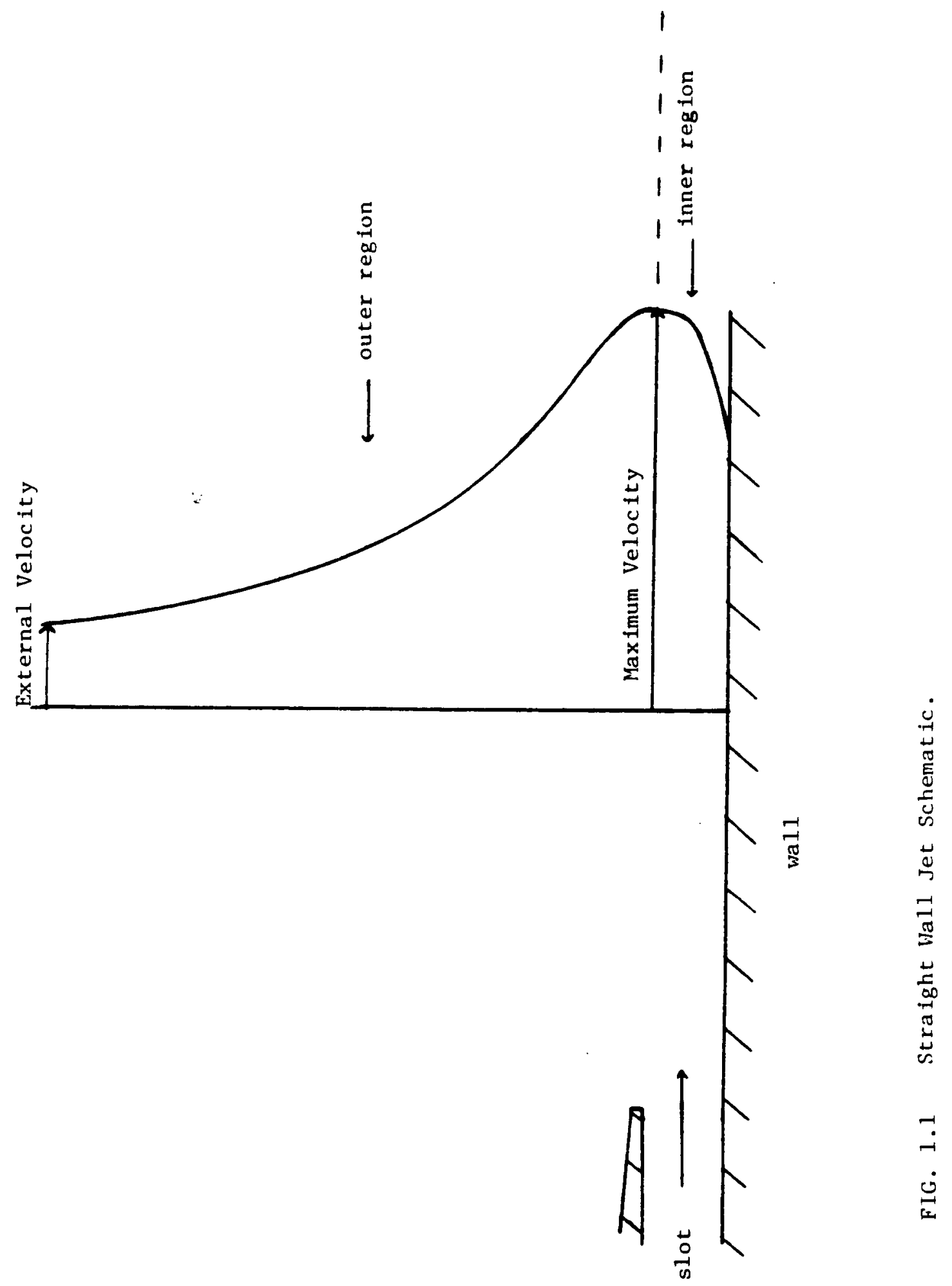




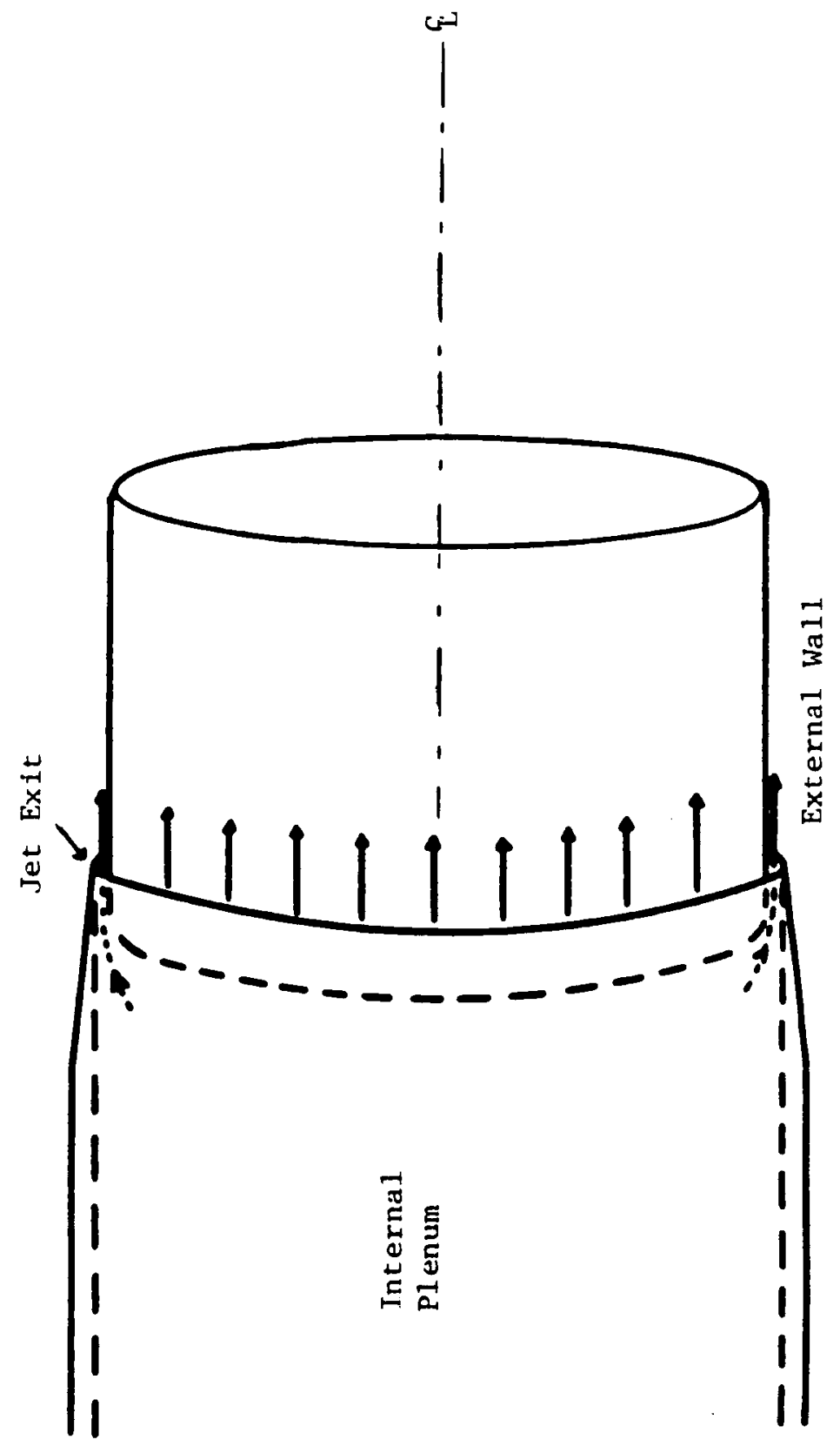

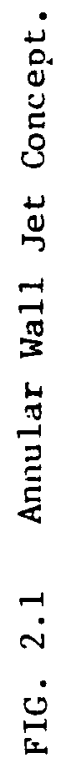




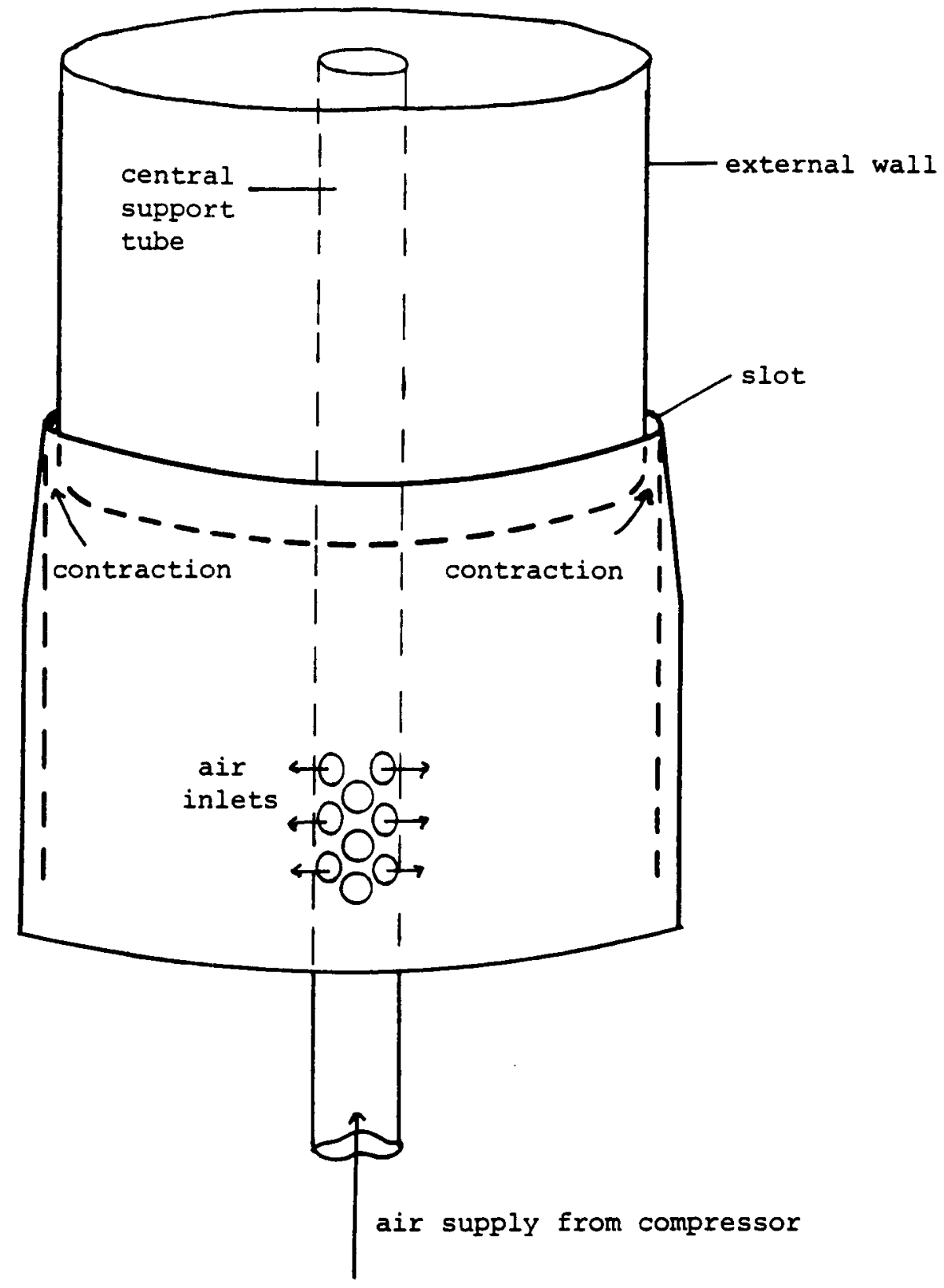

FIG. 3.1 Annular Wall Jet Model. 

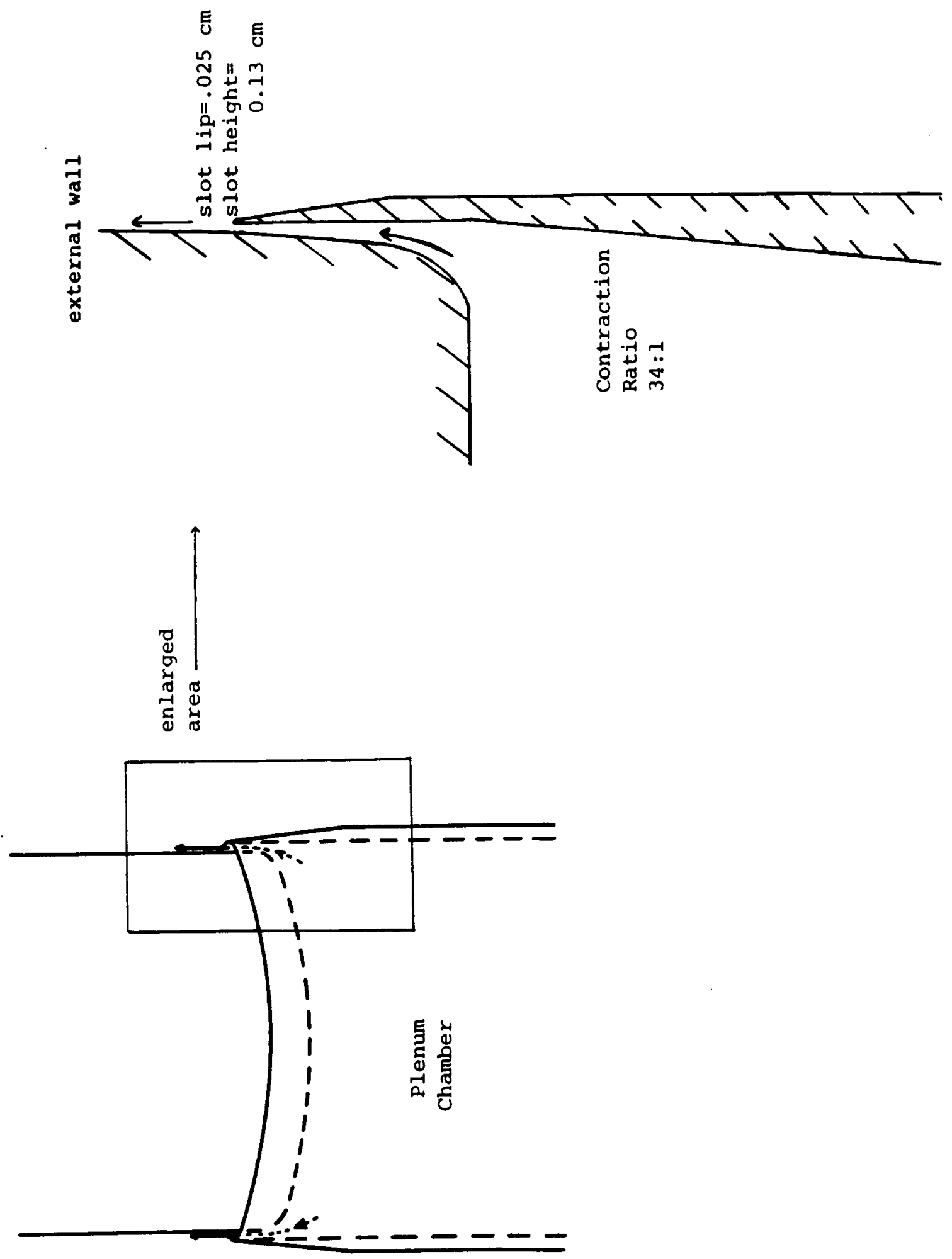

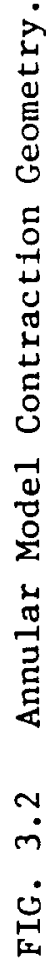



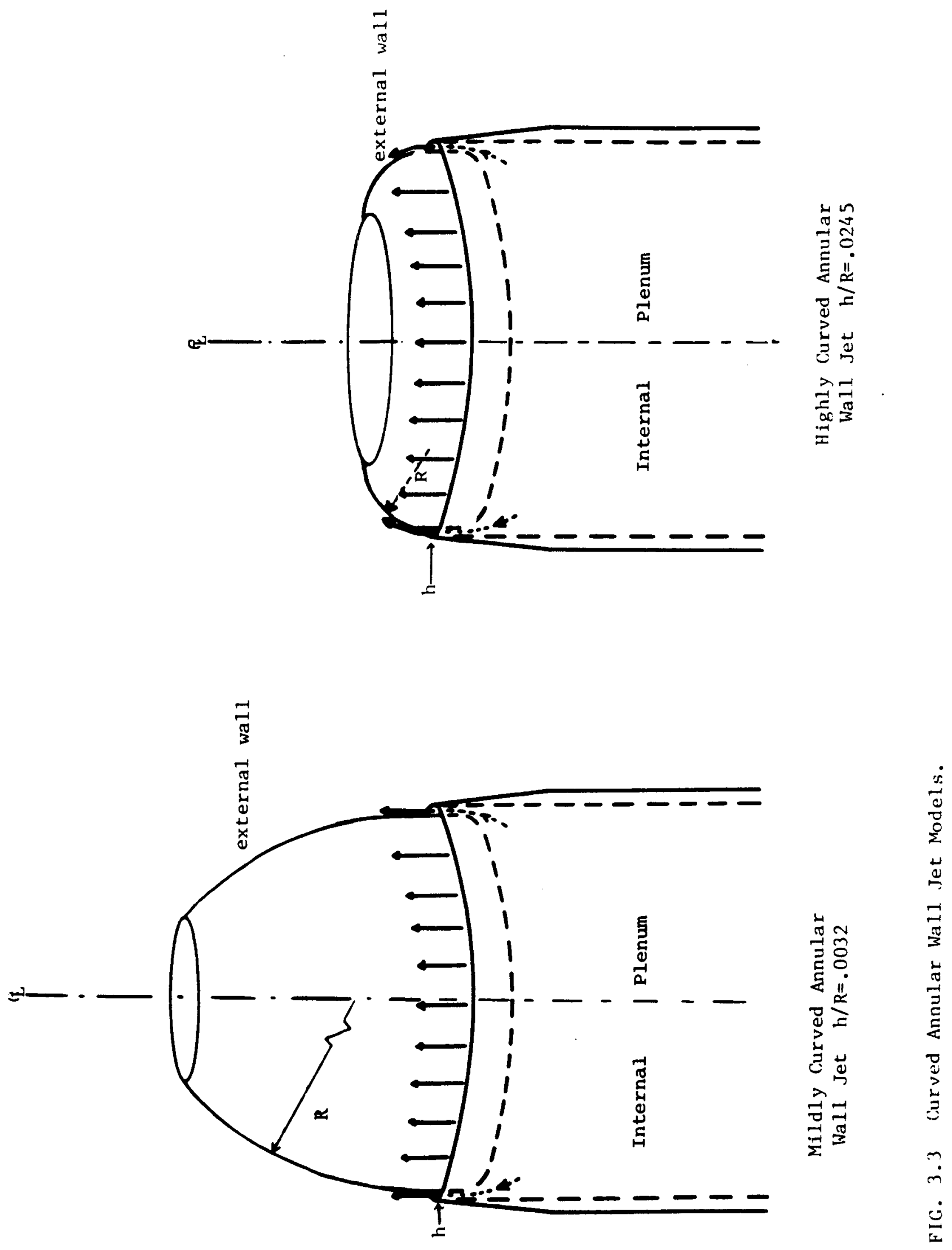


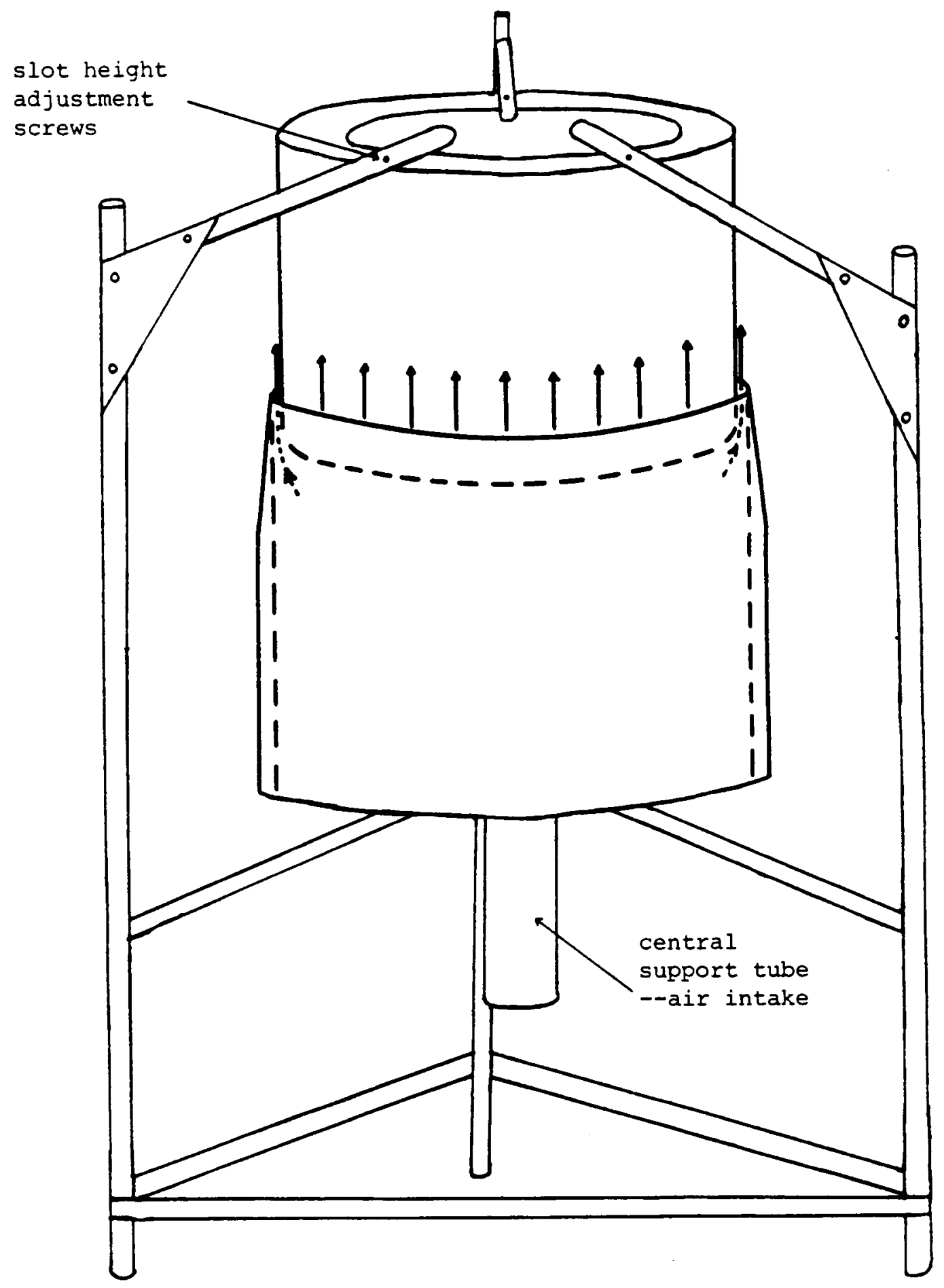

FIG. 3.4 Support Stand for Annular Wall Jet Model. 


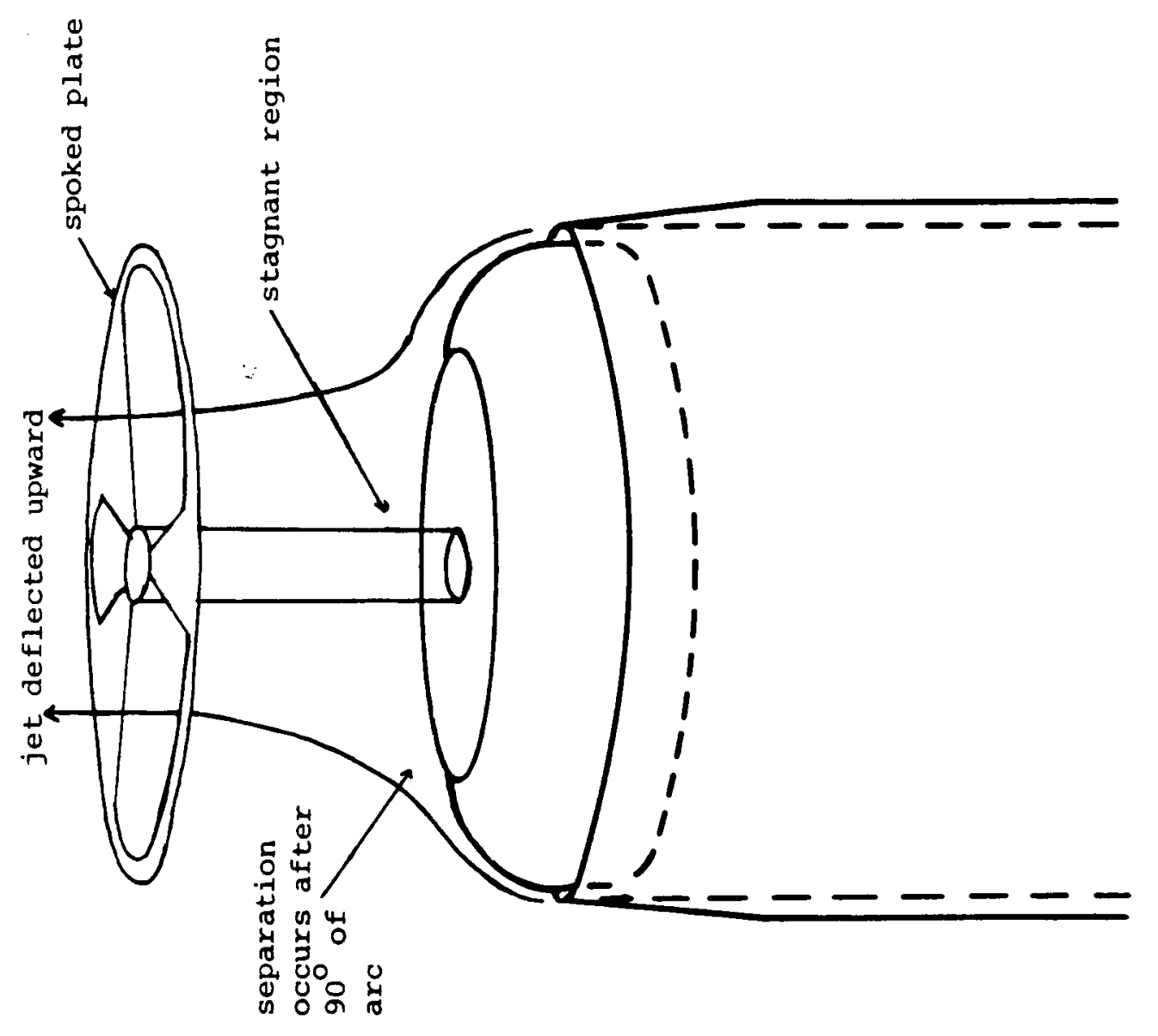

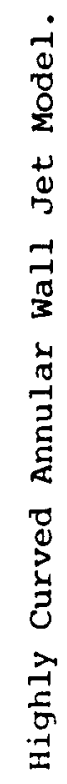

$n$
$\dot{n}$
$\dot{\Xi}$ 


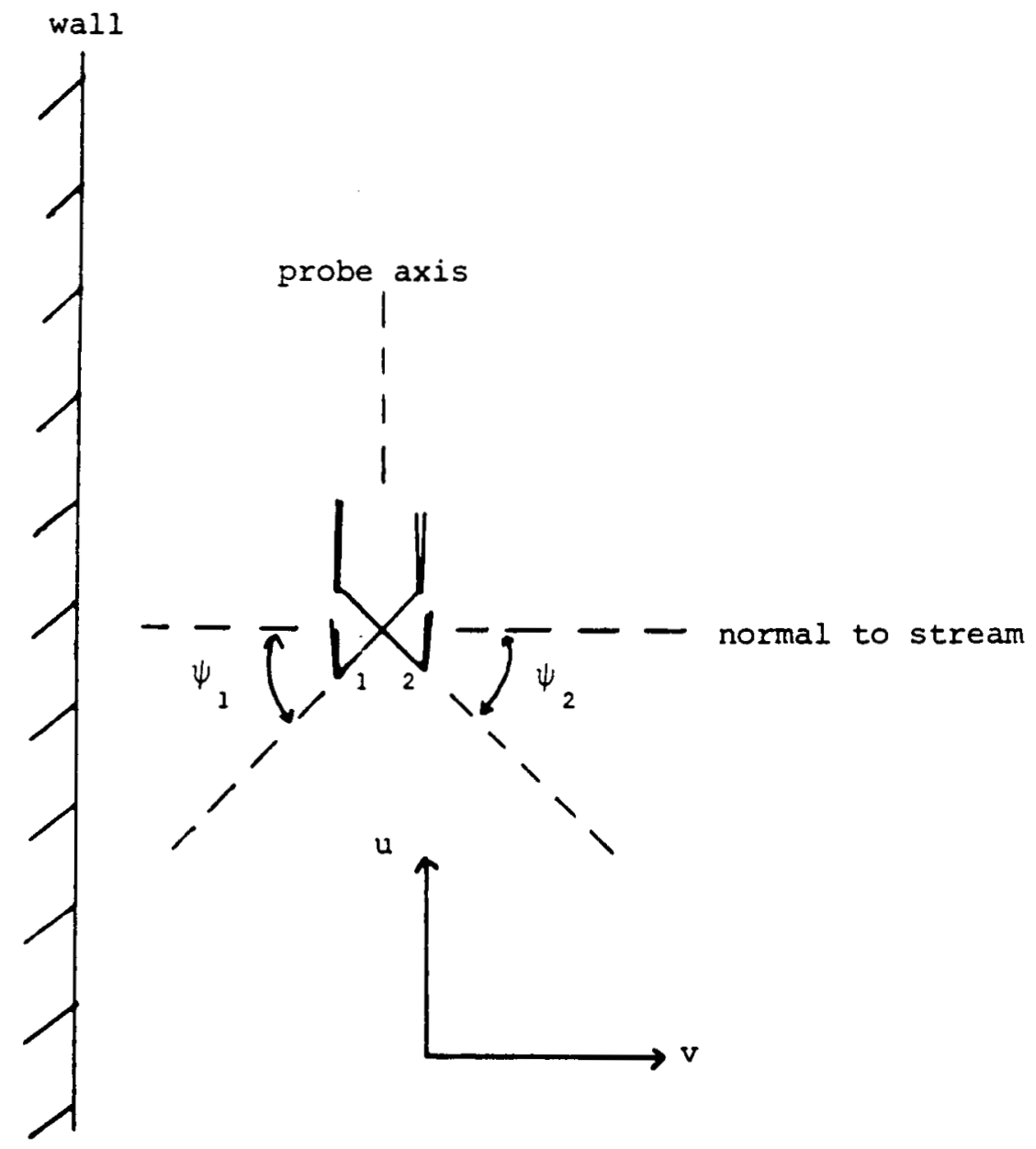

FIG. 3.6 Cross-Wire Probe Orientation. 


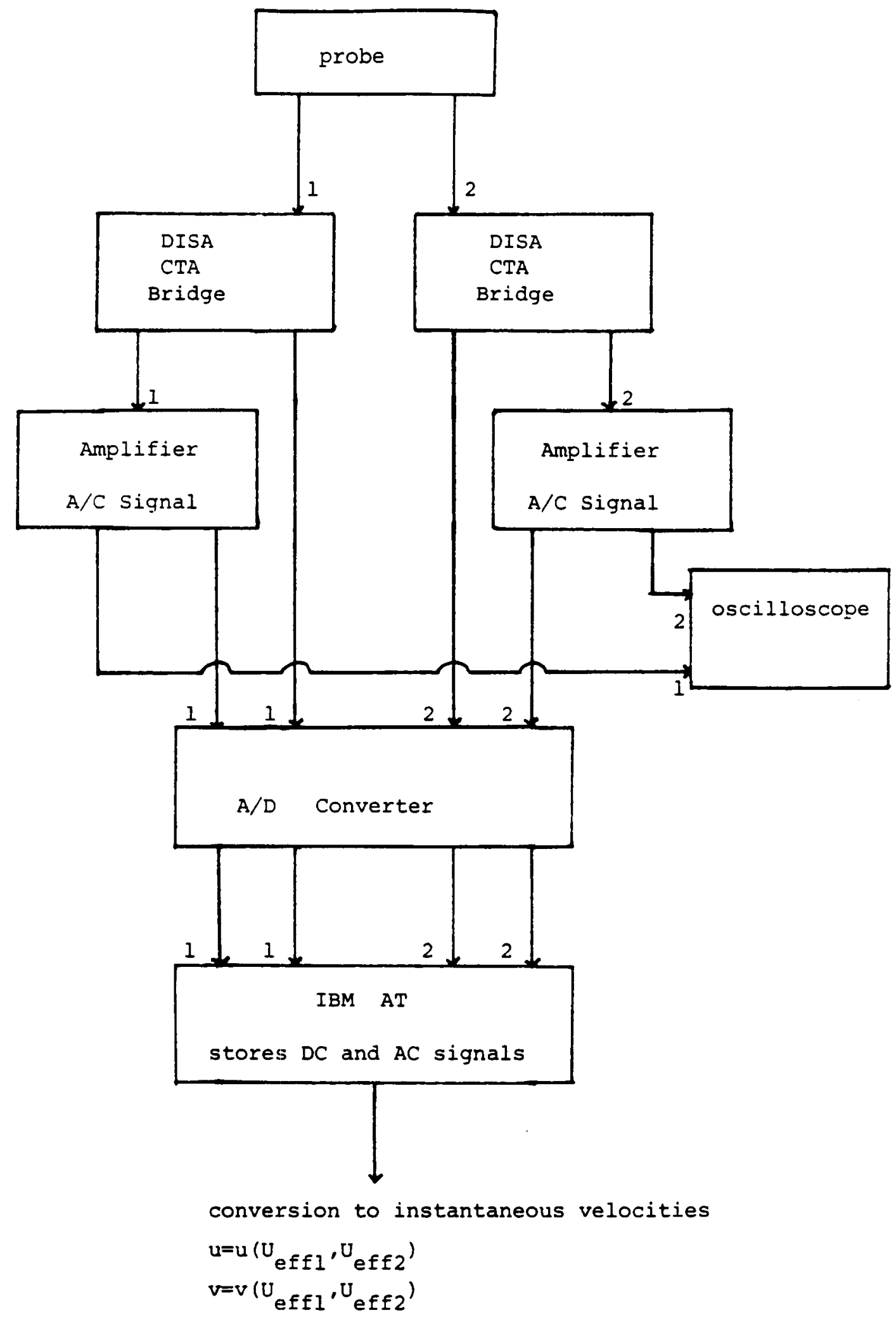

FIG. 3.7 Flowchart of Cross-Wire Operation. 


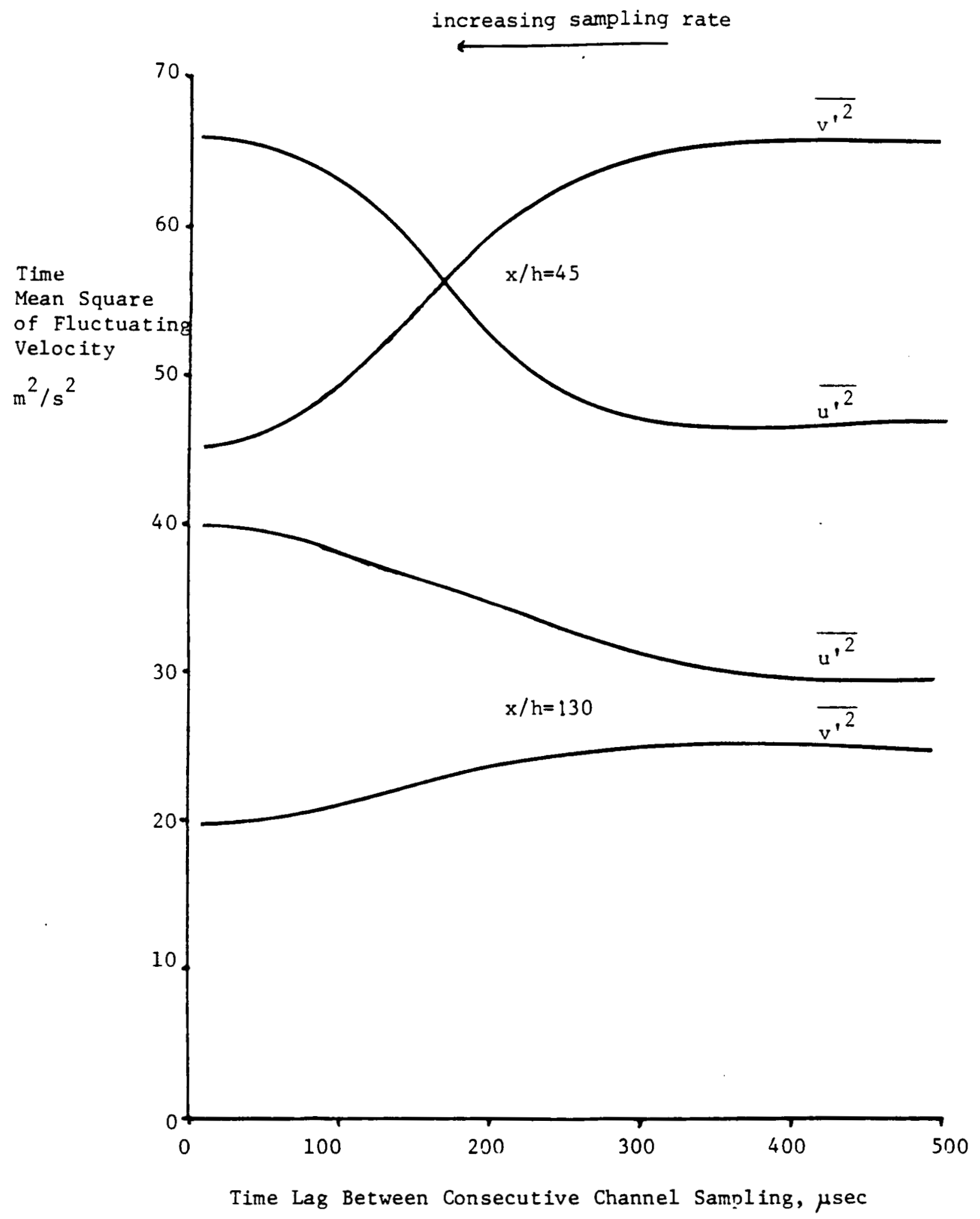

FIG. 3.8 Effect of Two-Channel Time Lag on Cross-lire Turbulence zasurements. 


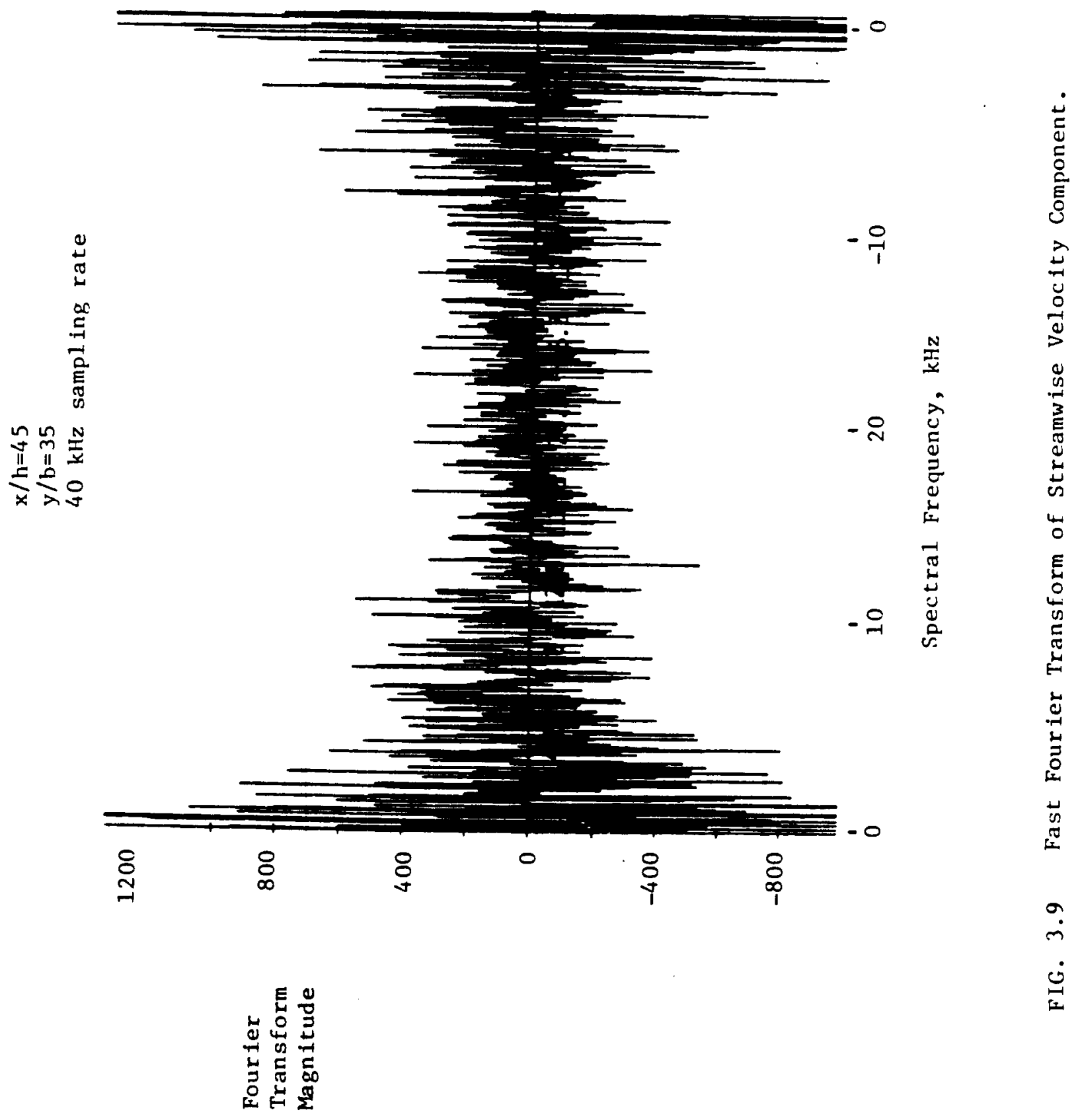




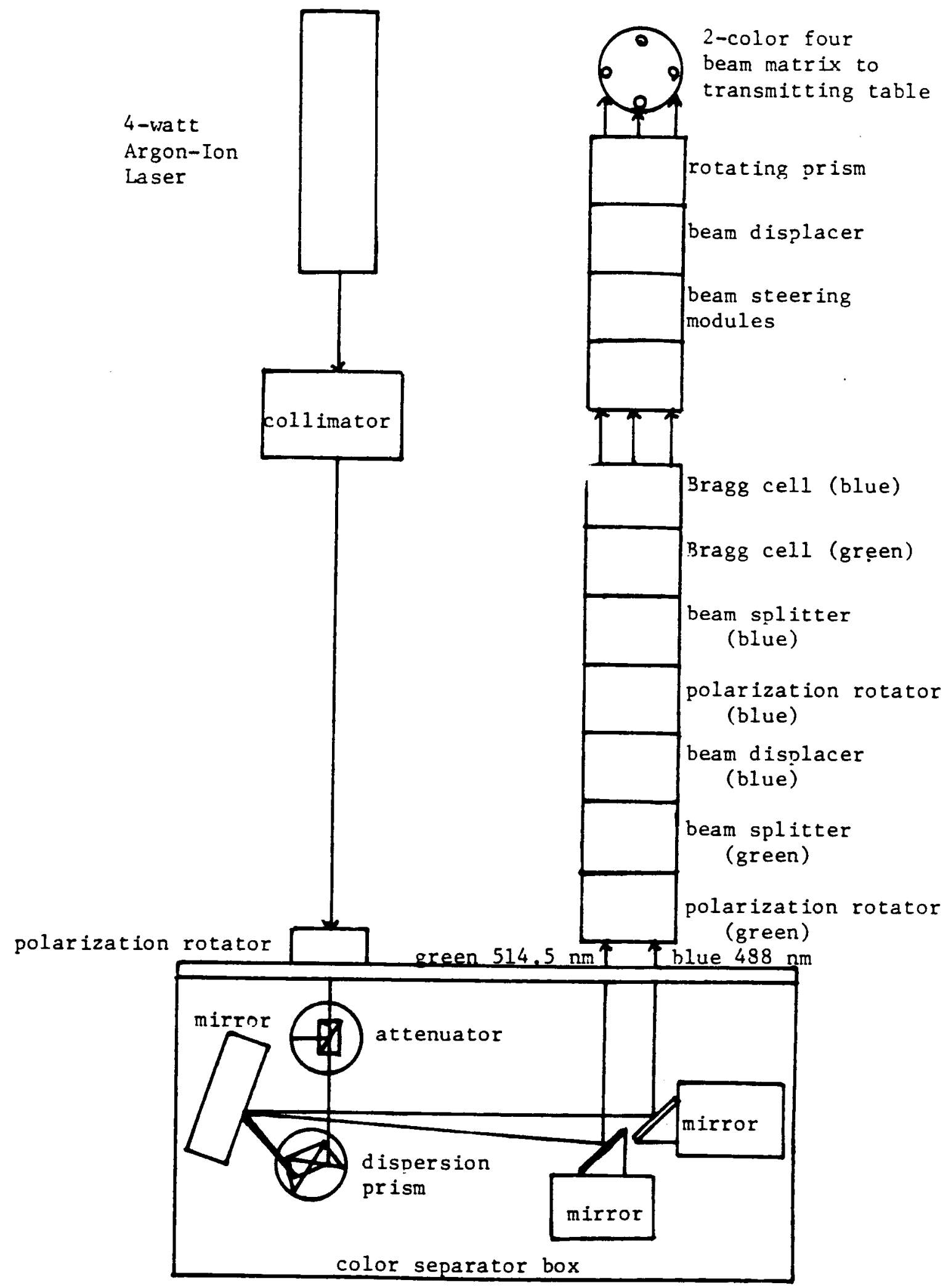

FIG. 3.10 Schematic of the Optics Table Layout. 


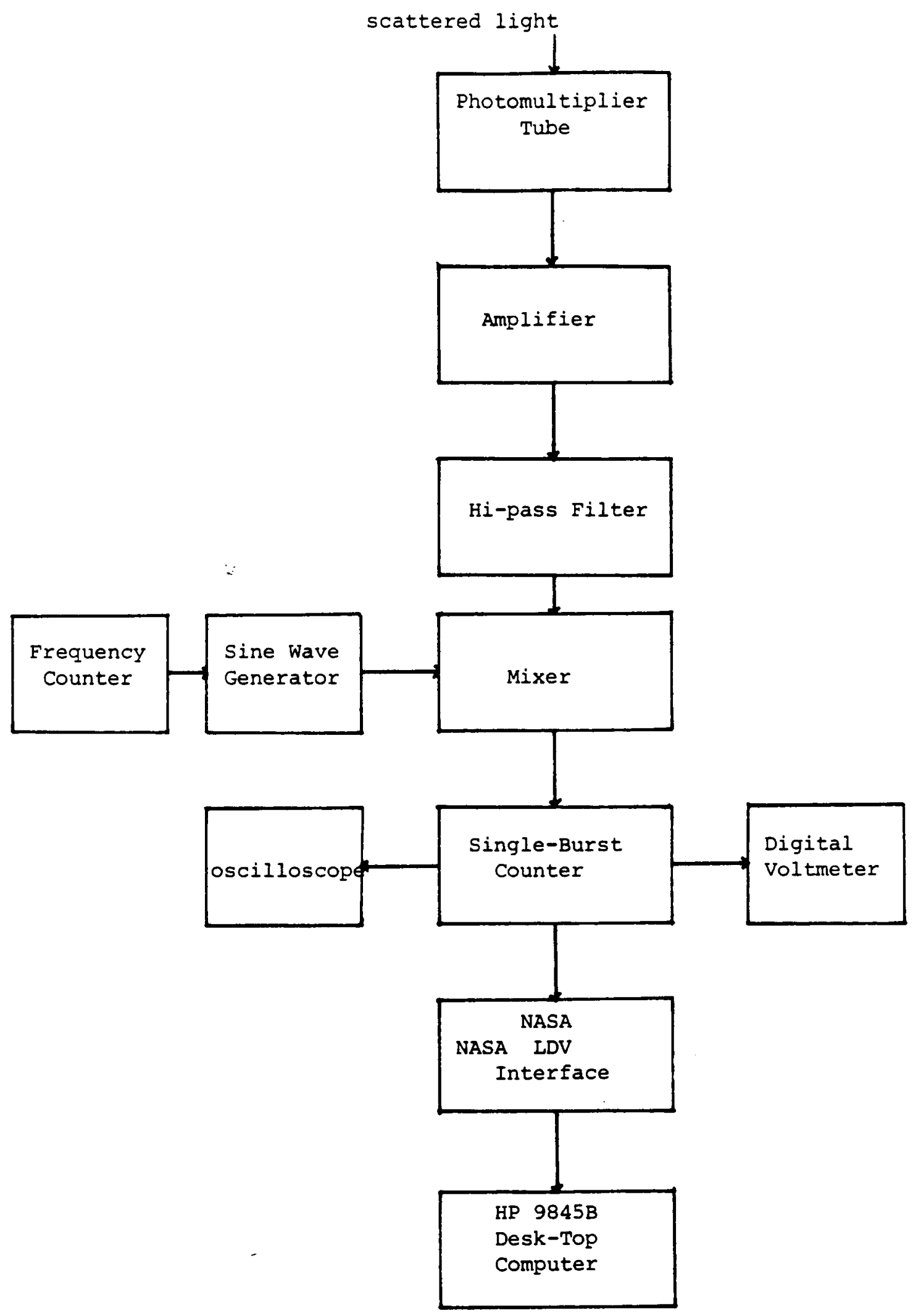

FIG. 3.11 Flowchart of LDV Operation. 

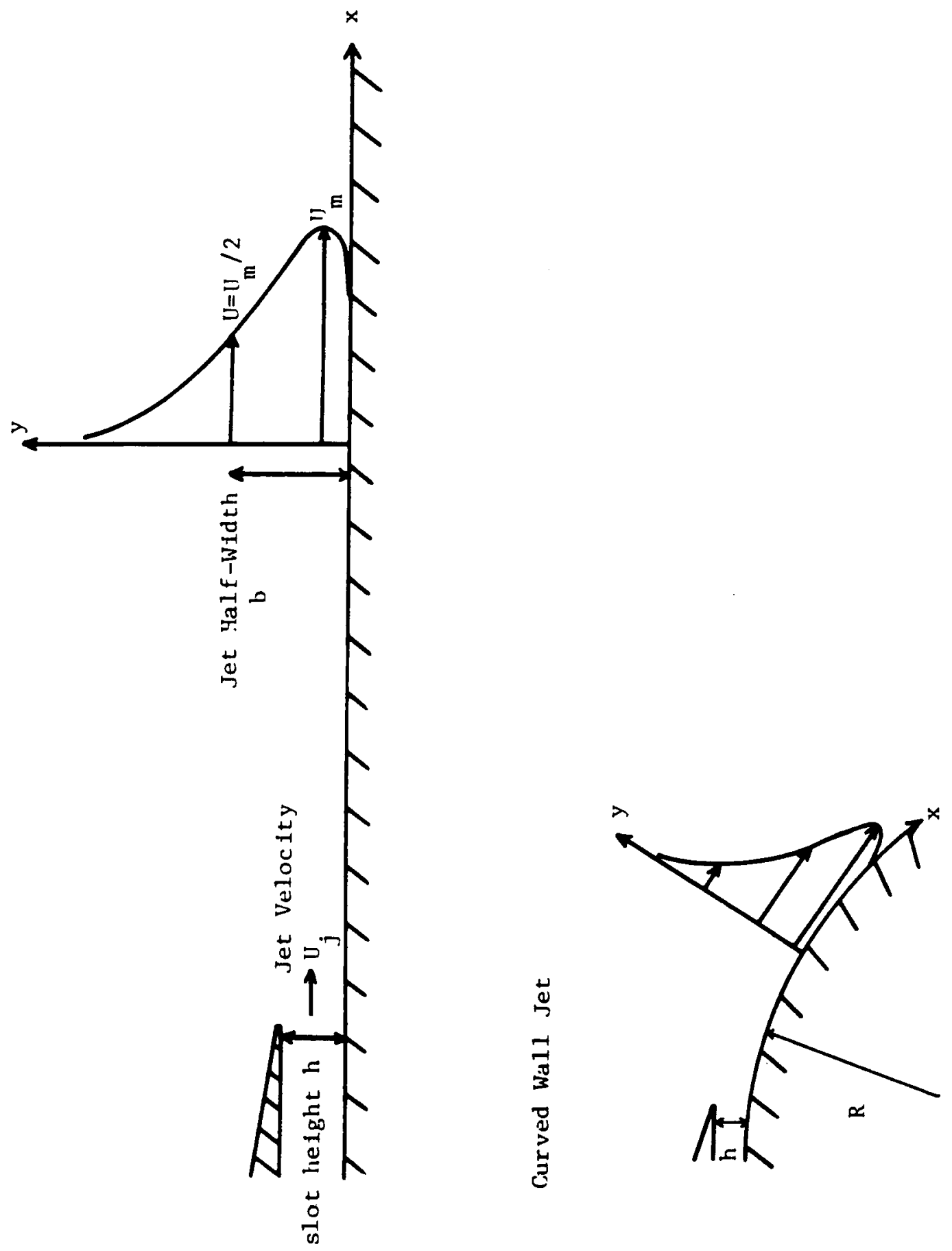

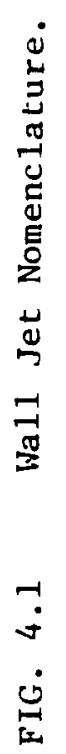




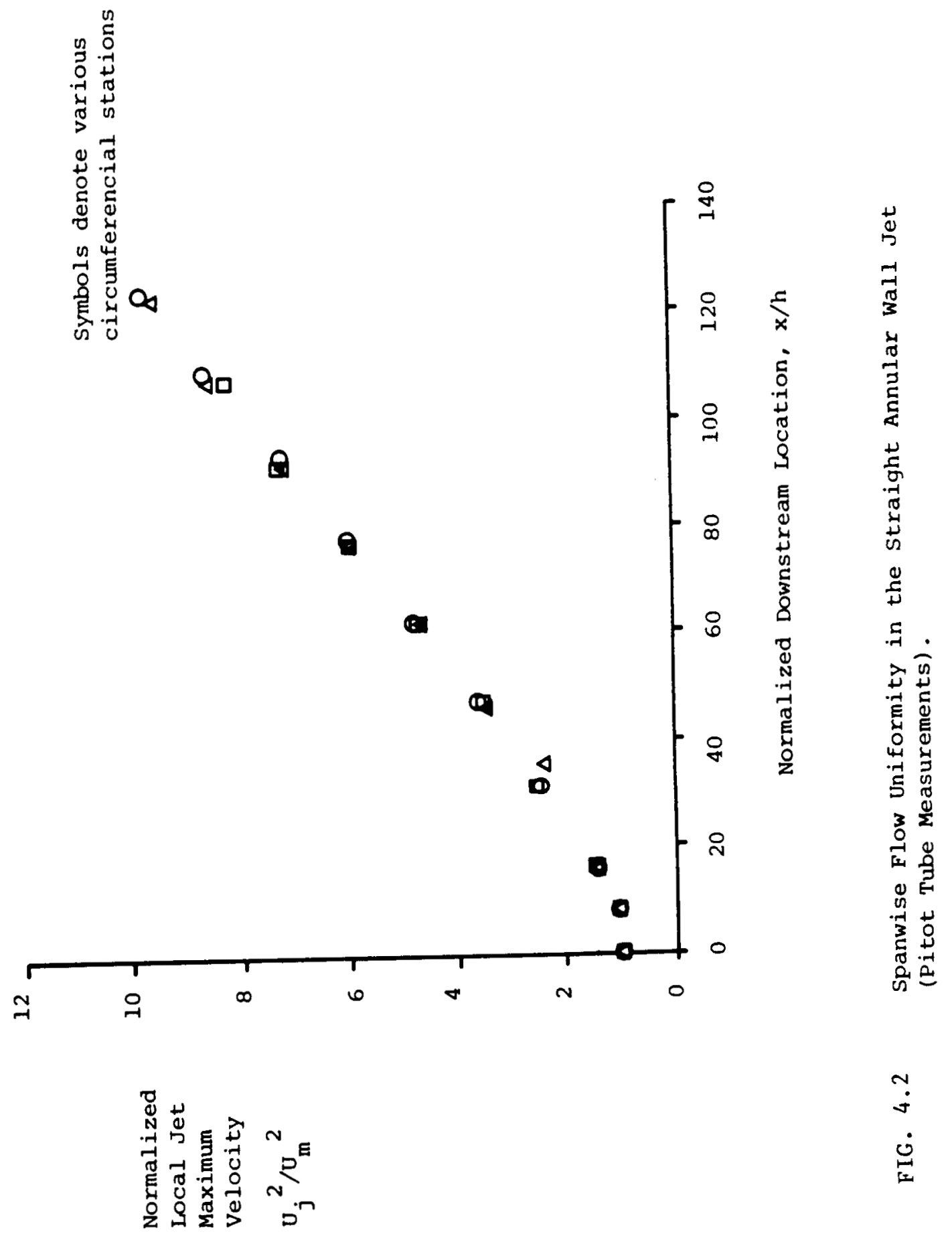




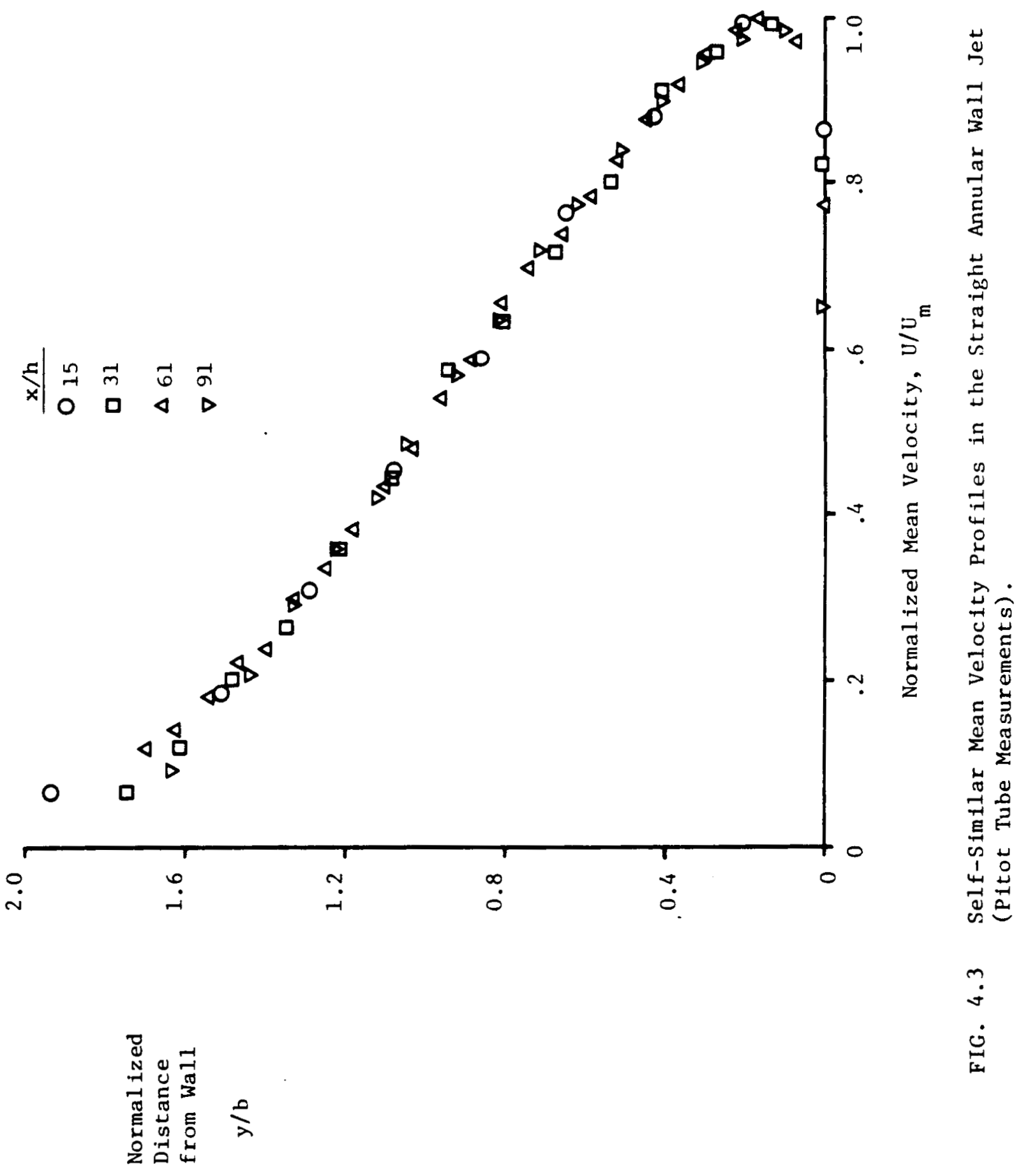




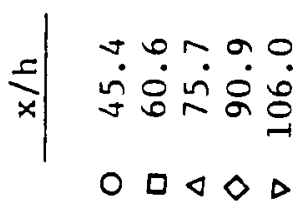

0
-1
$=$
3
3
3
3
3

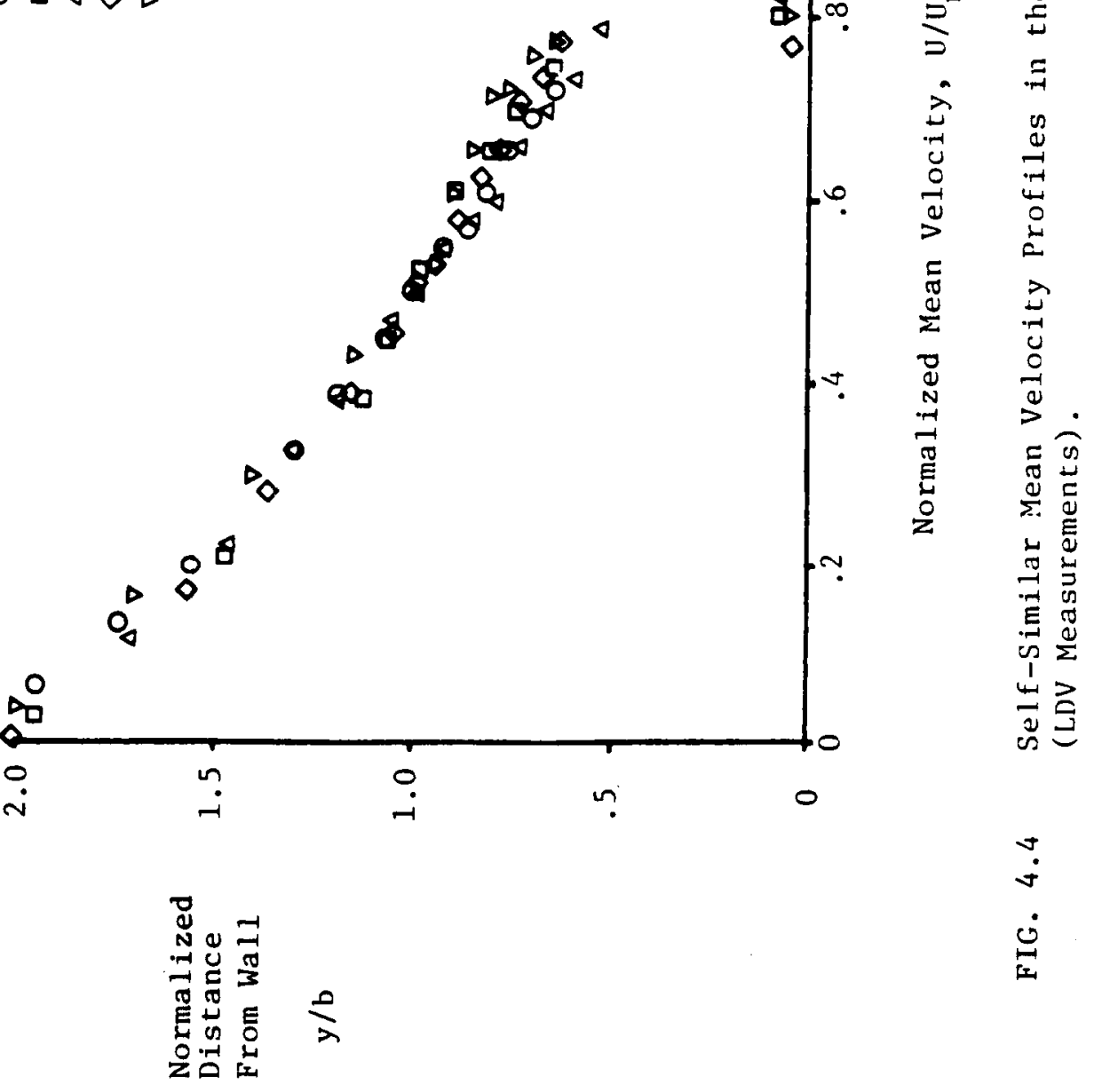




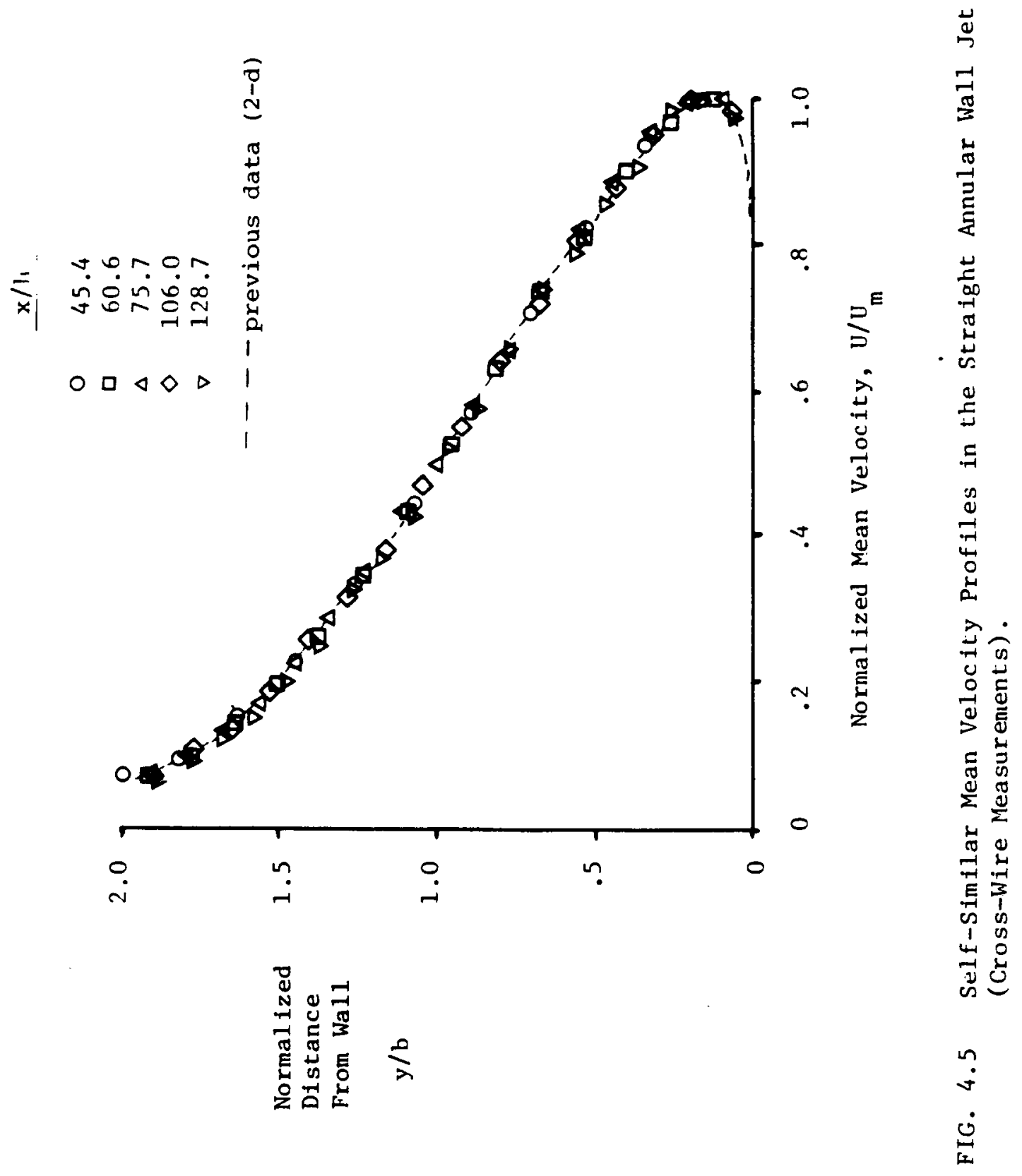




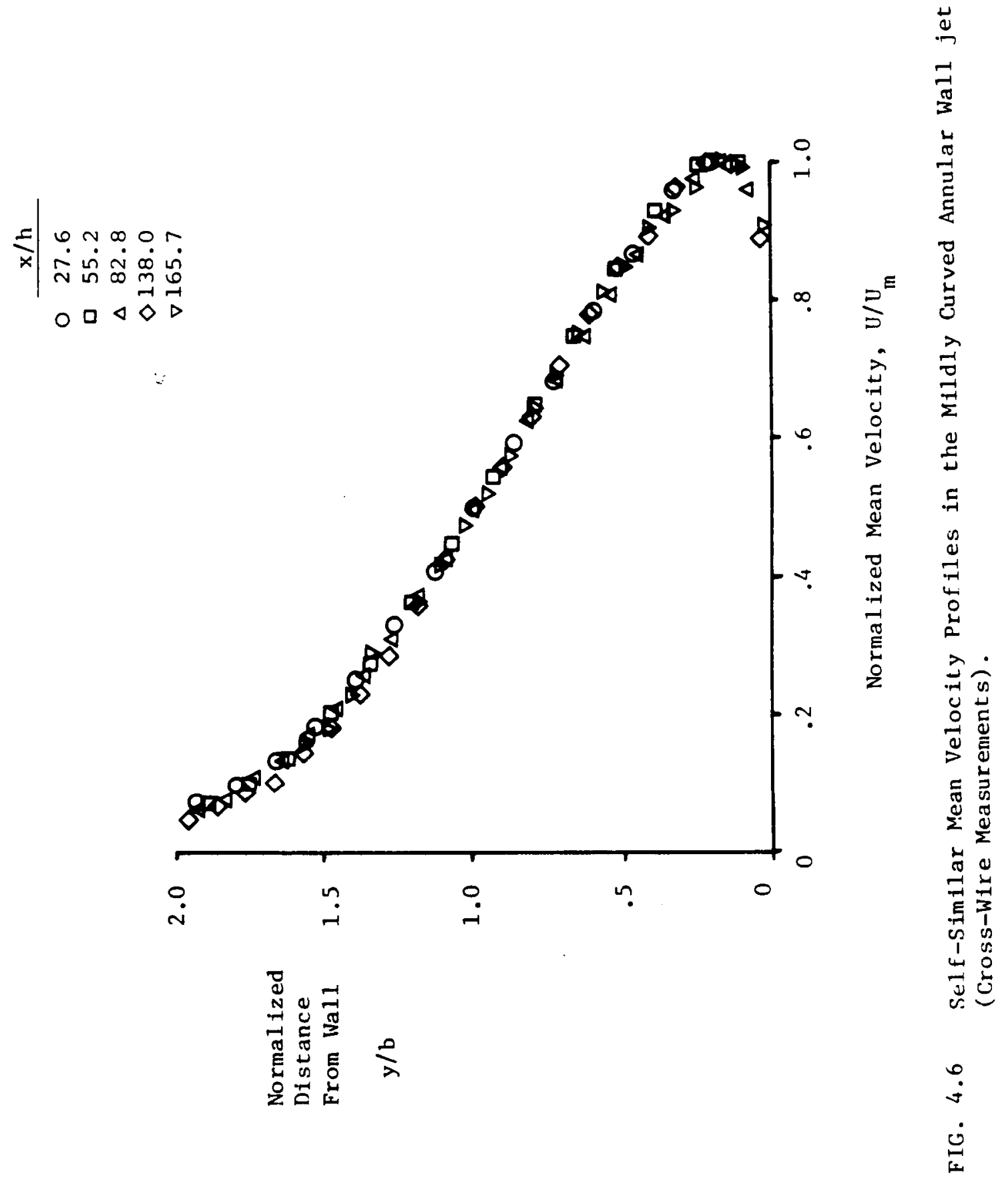




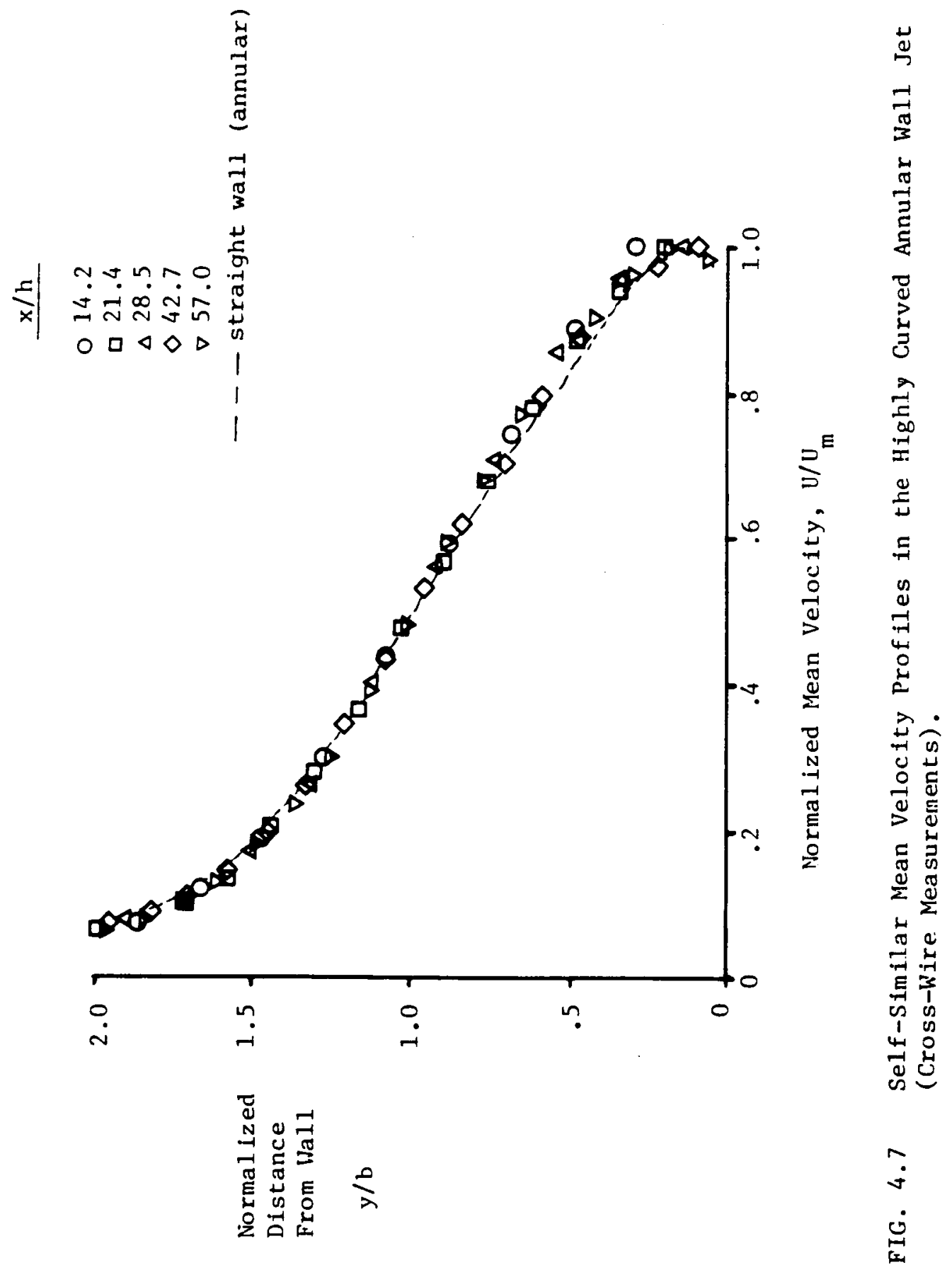




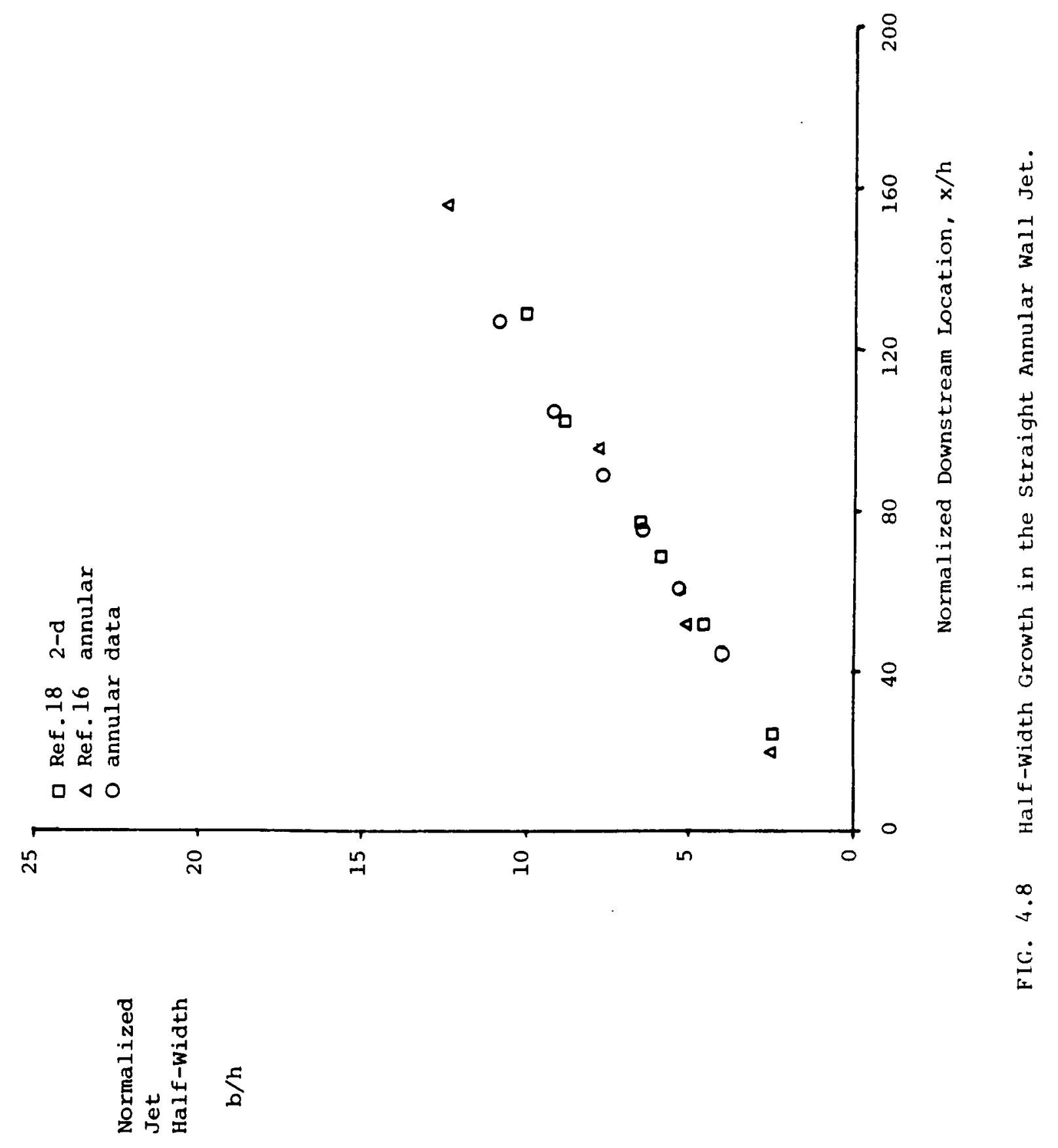




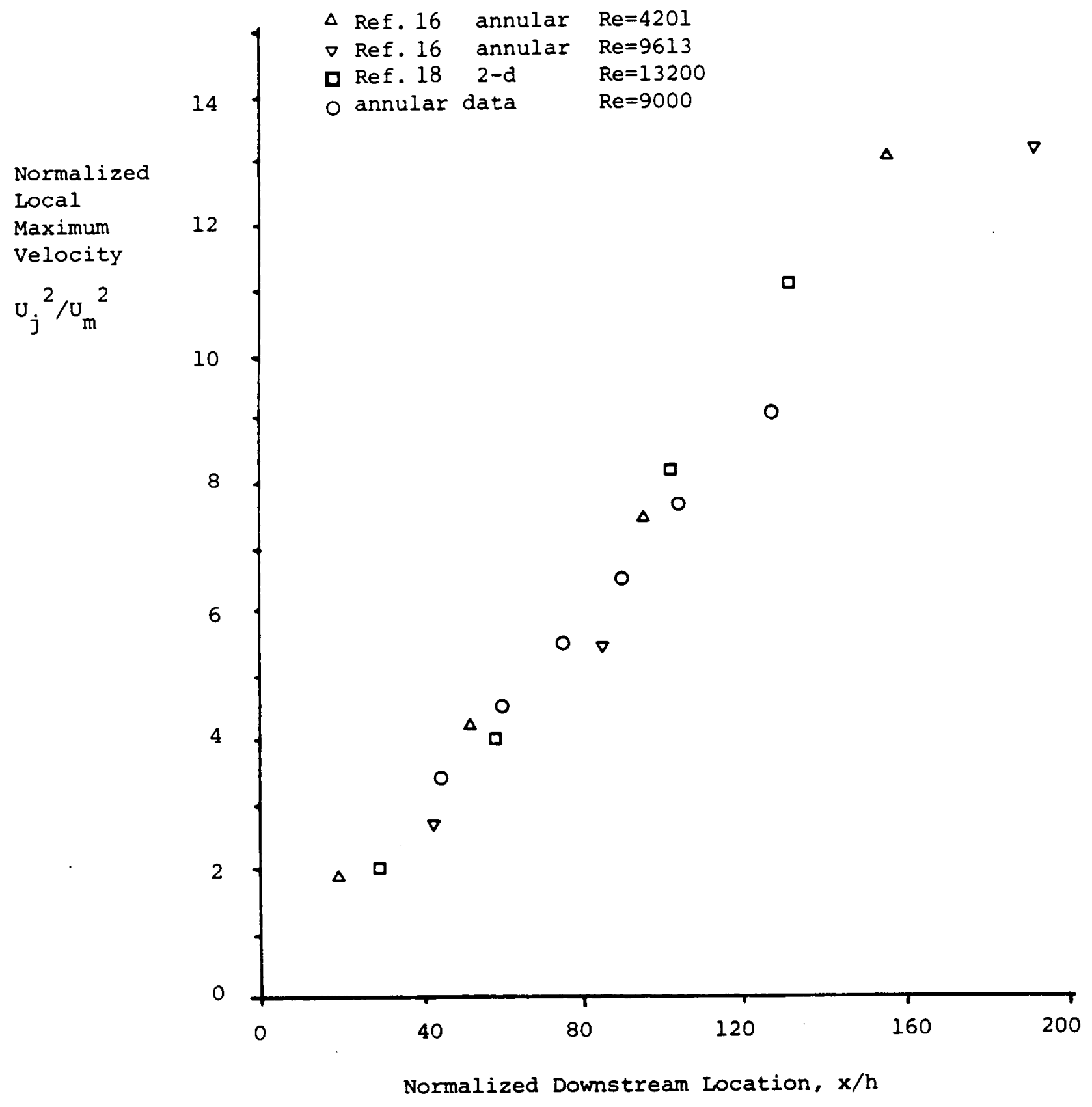

FIG. 4.9 Maximum Velocity Decay in the Straight Annular Wall Jet. 


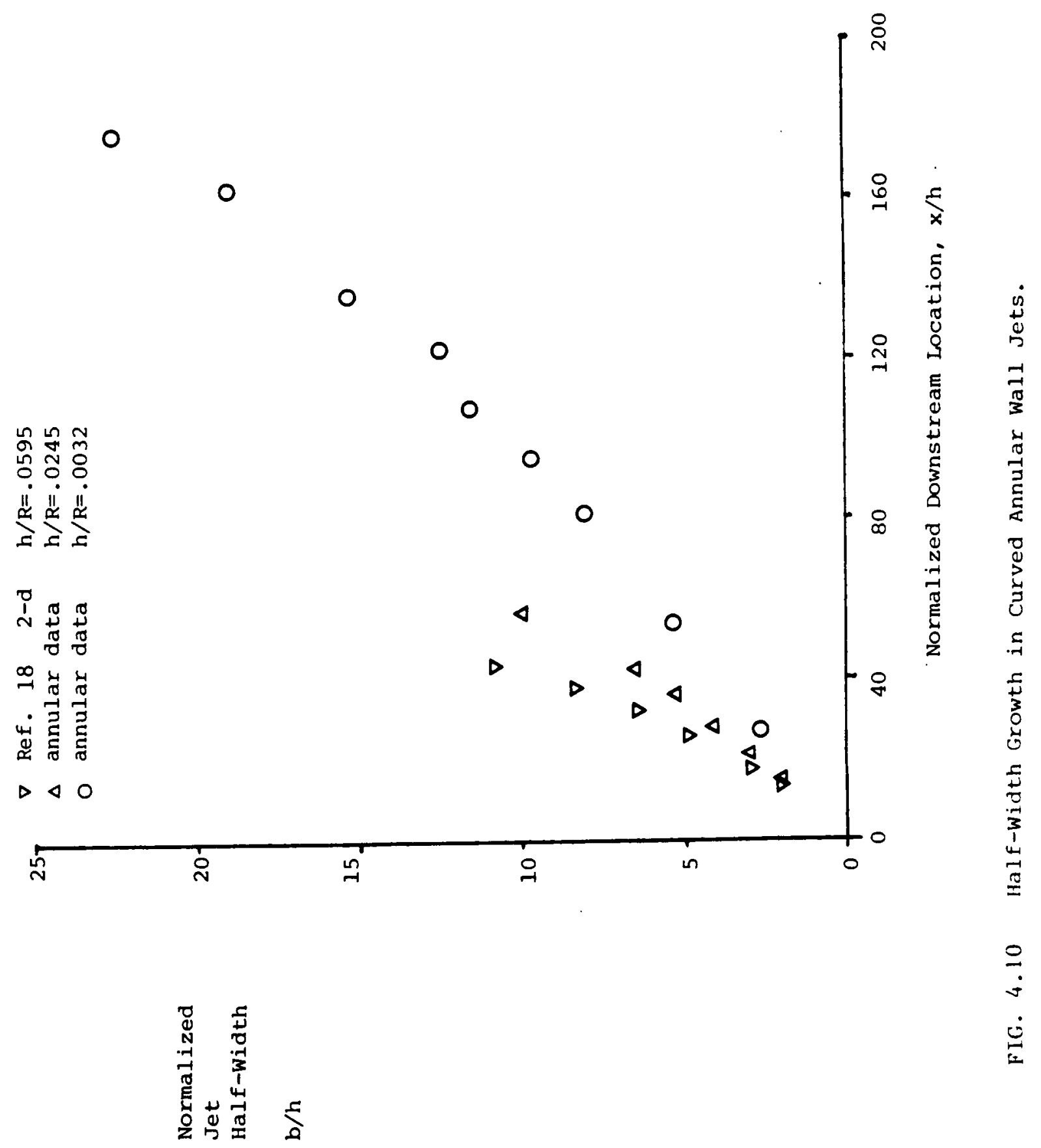


ตㄴ

กั

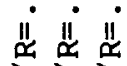

0

टे

0

$\stackrel{i}{i}$

赵苗

0

$\triangle \triangleleft 0$

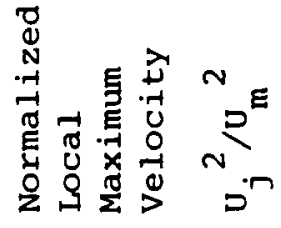

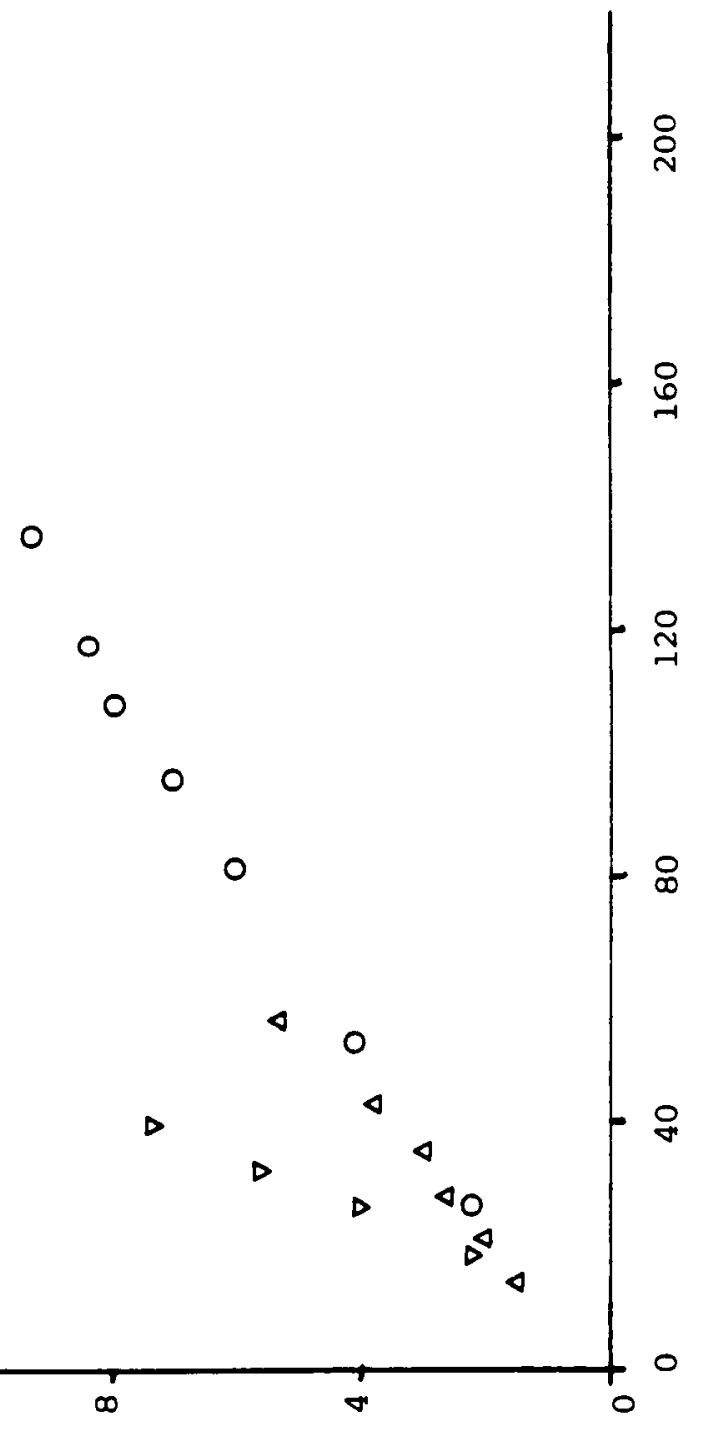

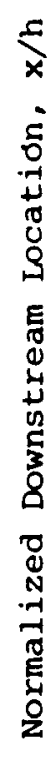

蓠

$\frac{7}{3}$

हี

离

.5

ฮั

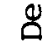

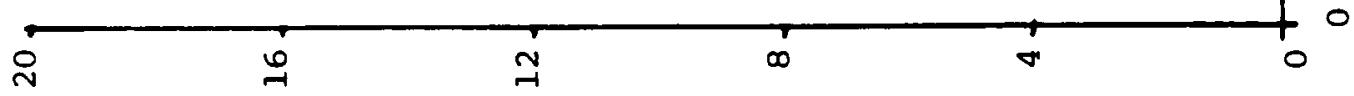

$\exists$

$\stackrel{9}{E}$ 


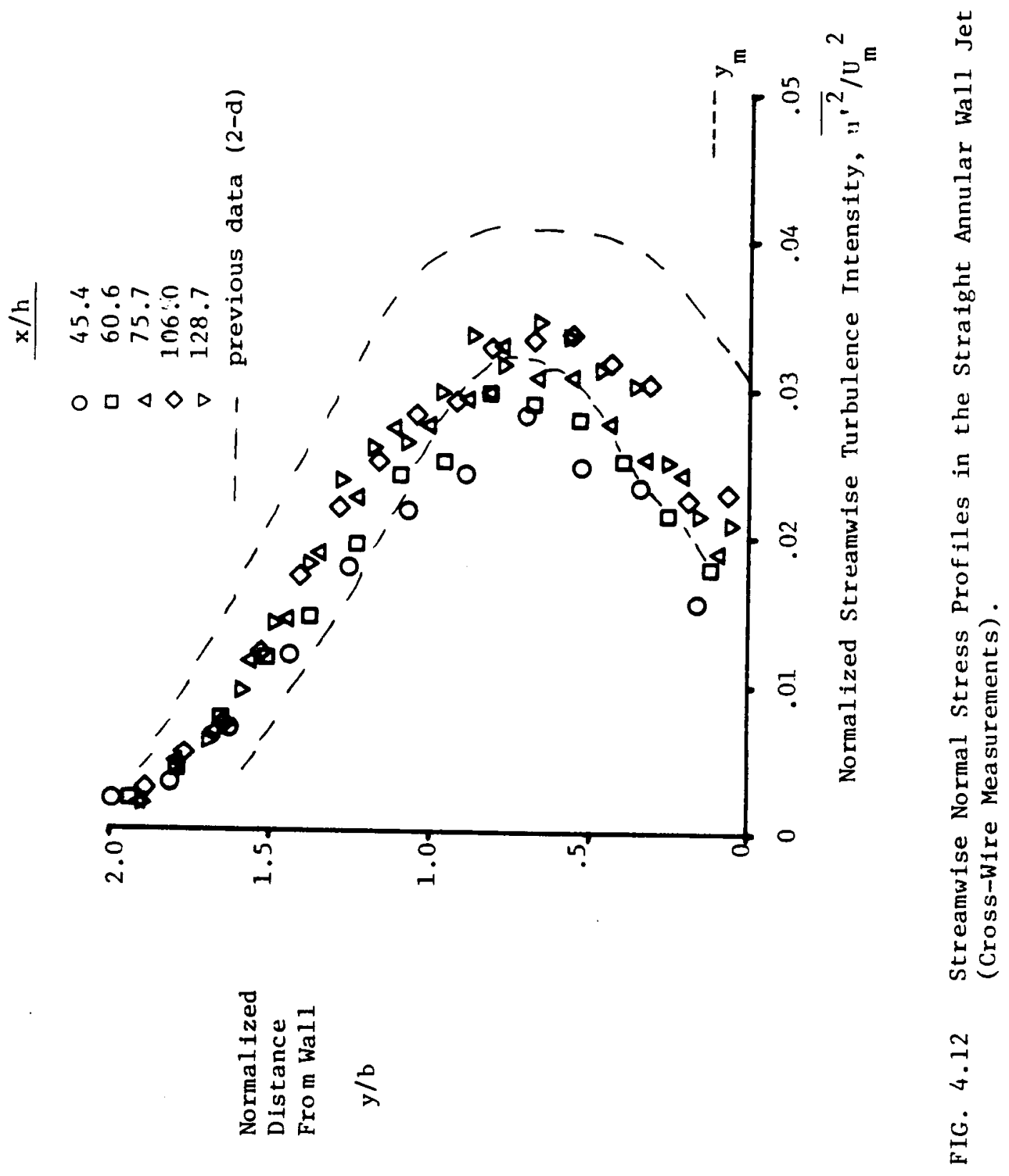




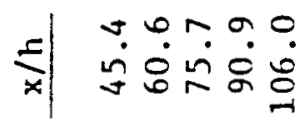

$0 \square \triangleleft \Delta D$

$\stackrel{\dddot{\Xi}}{\sim}$
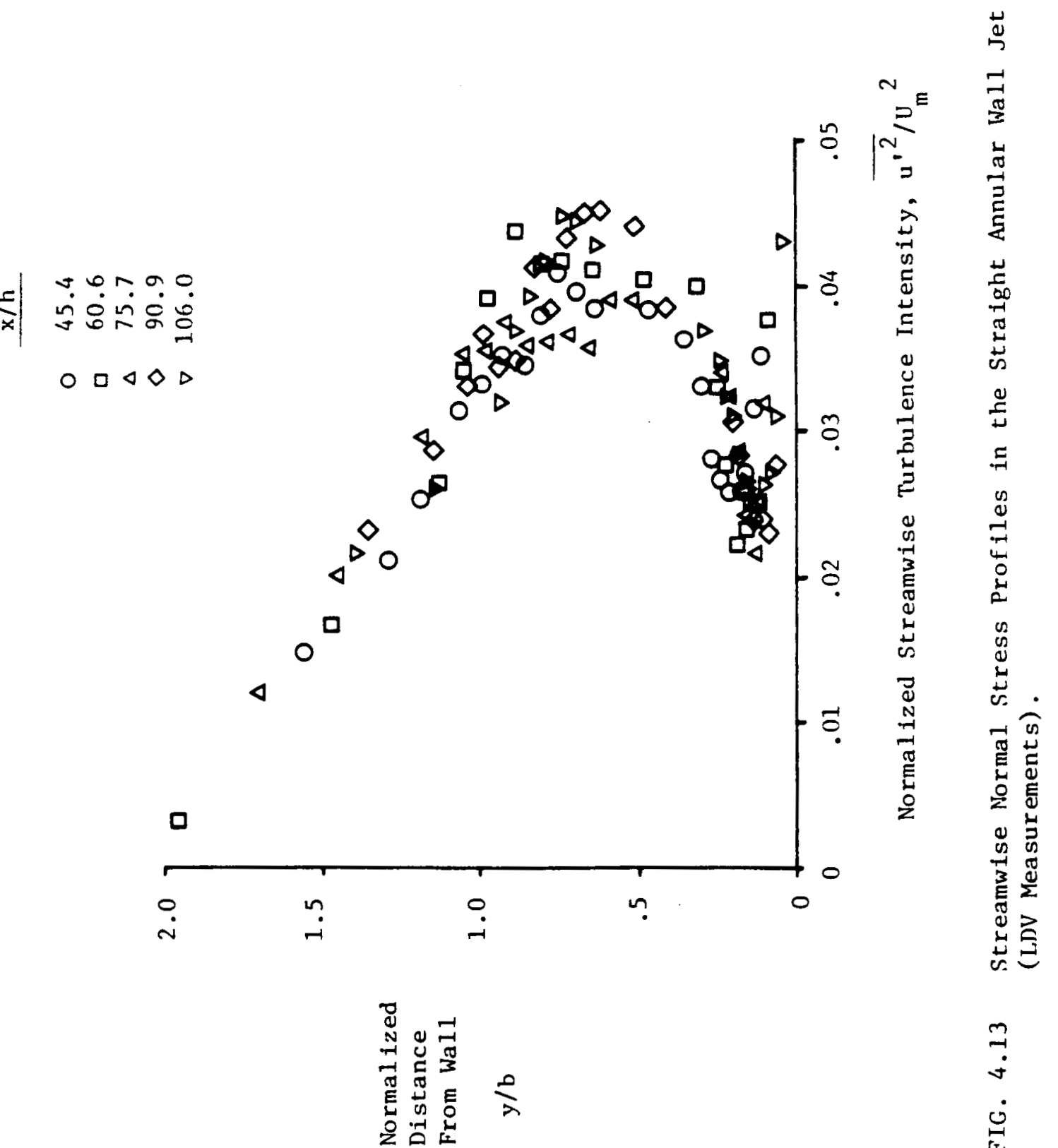

$\stackrel{m}{-1}$
$\dot{y}$
$\dot{5}$ 


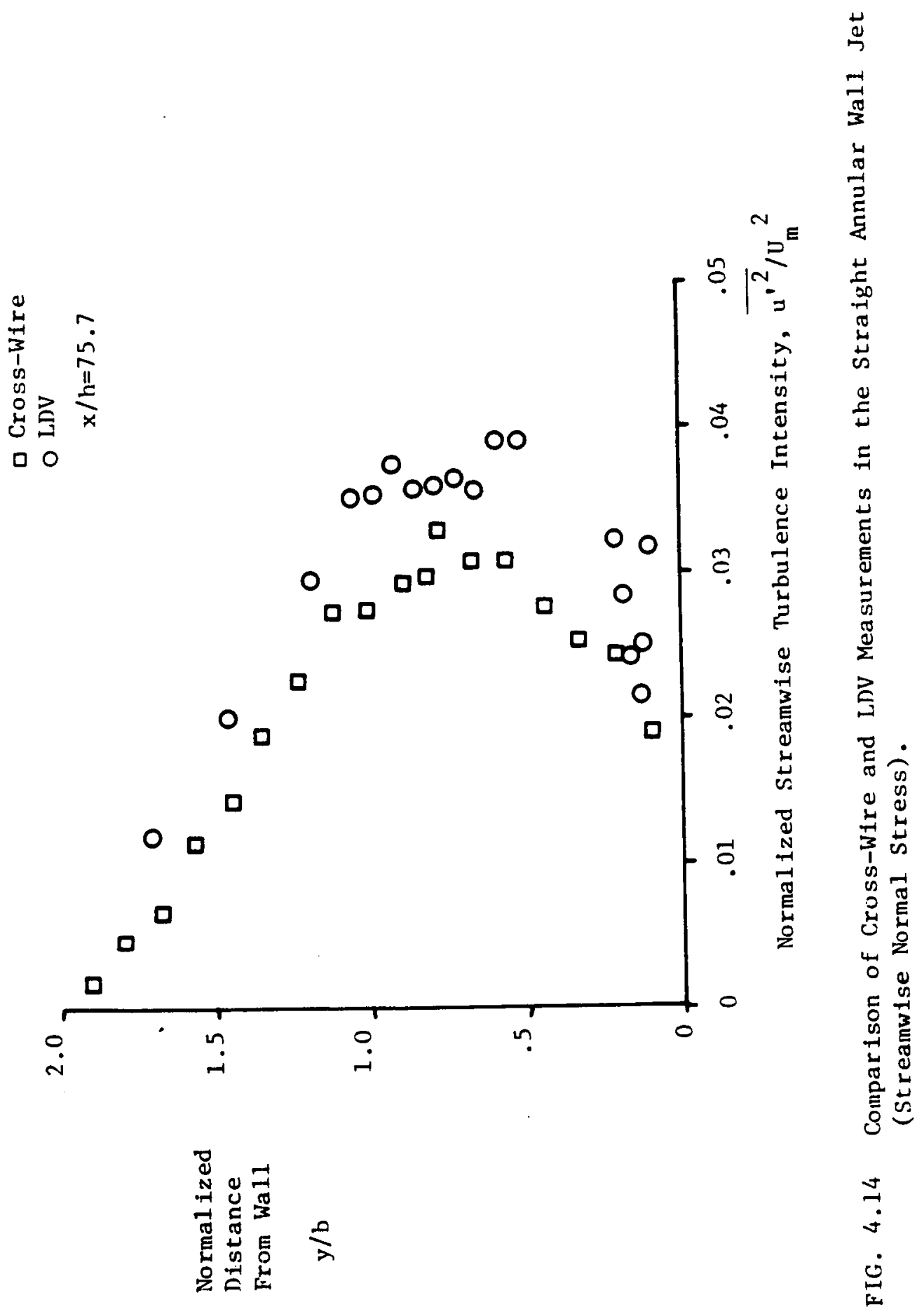




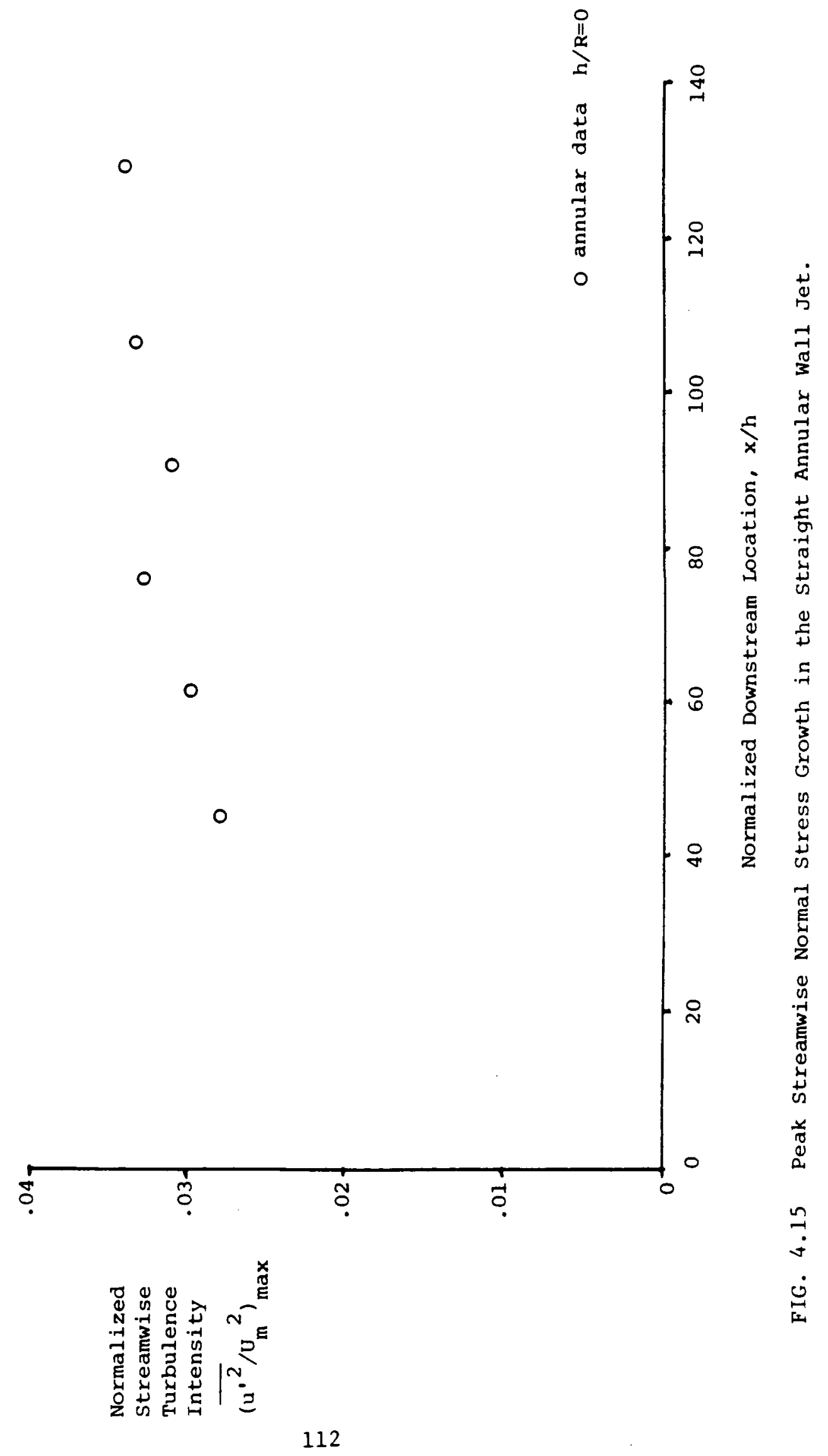




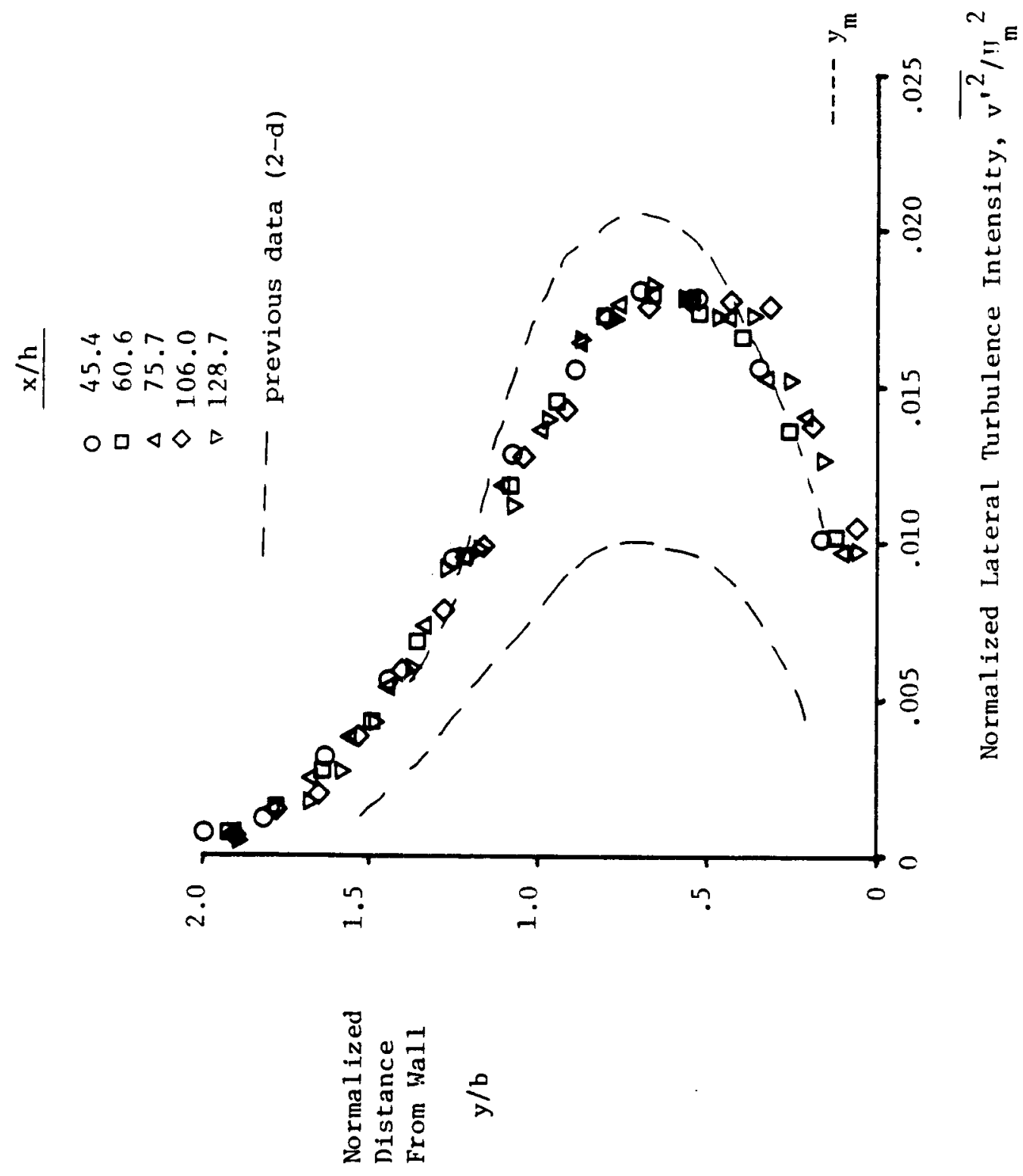

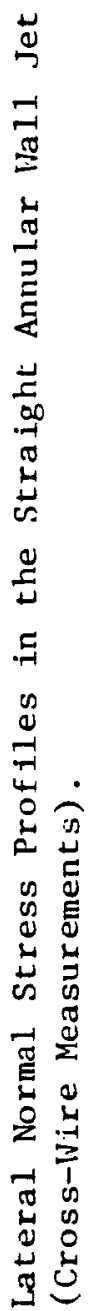

$\stackrel{0}{9}$

它 


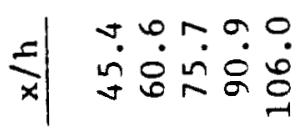

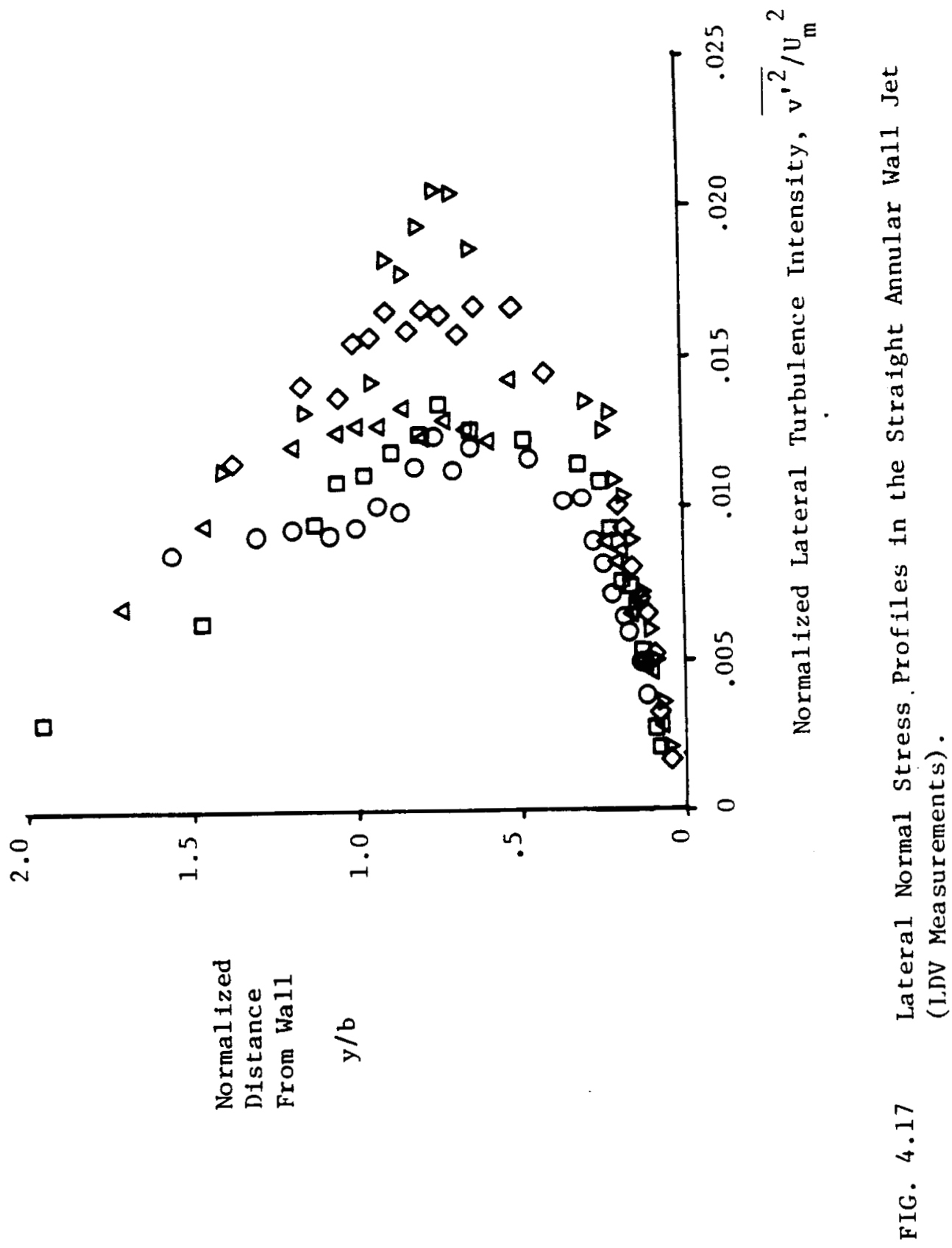




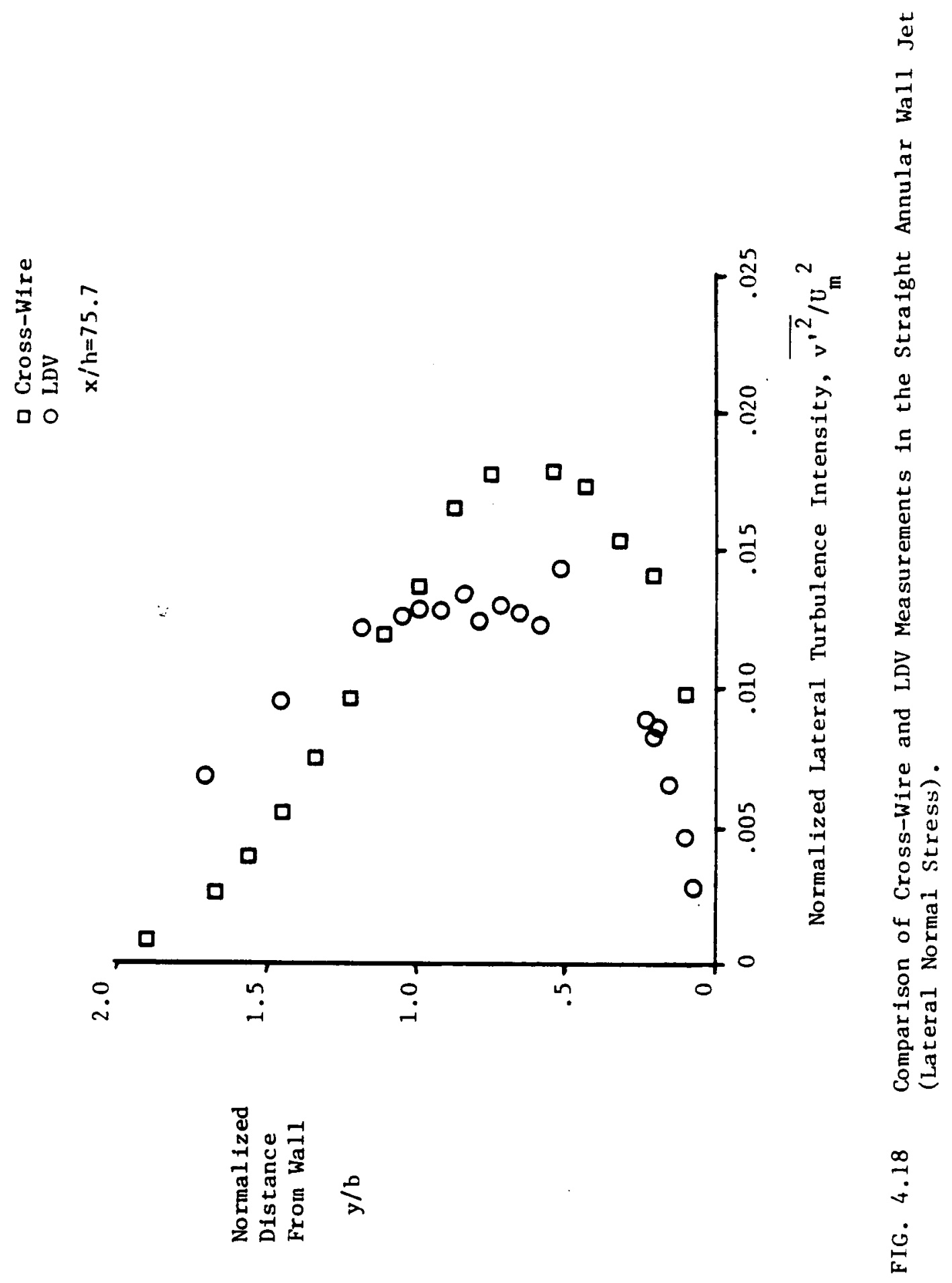




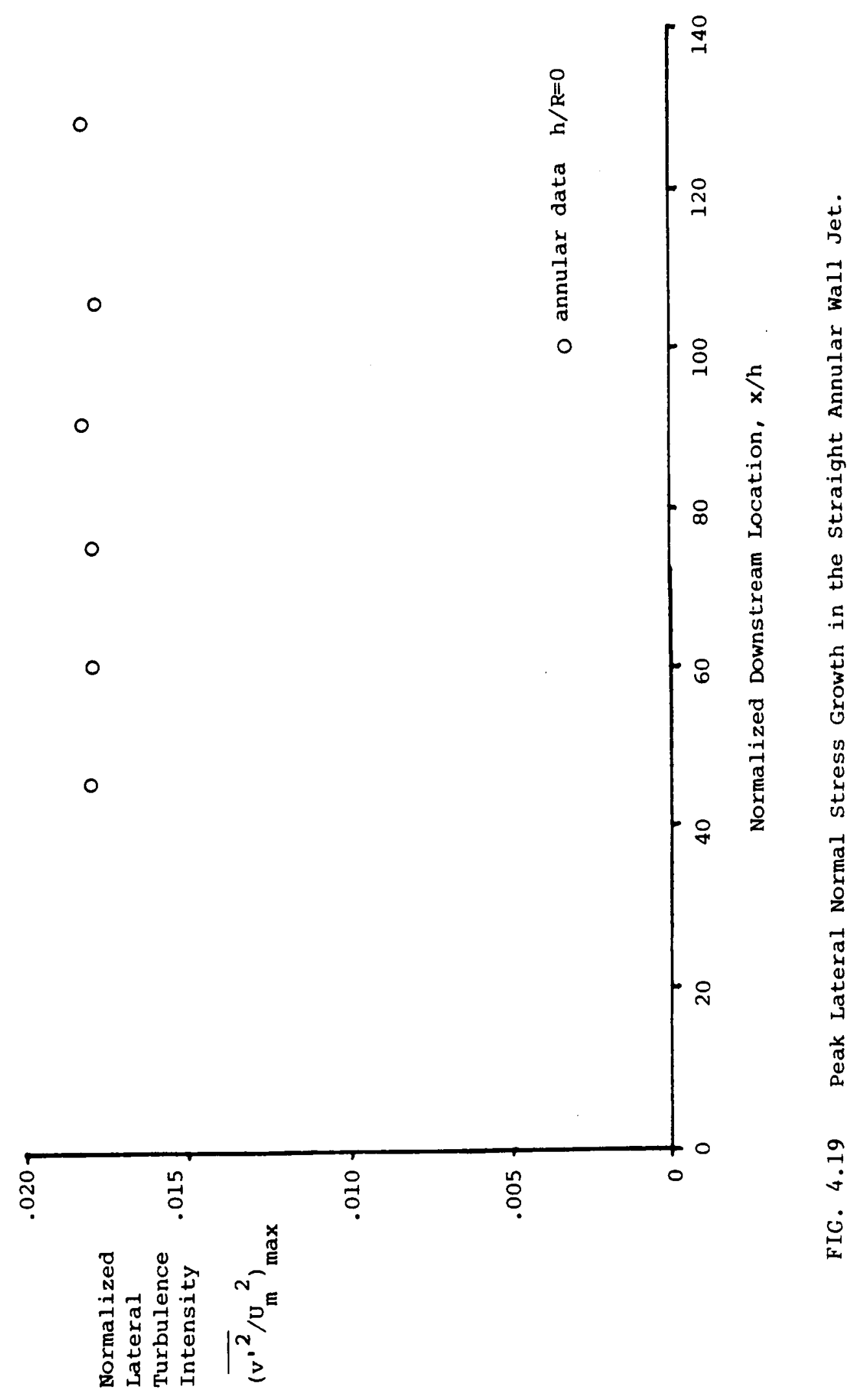




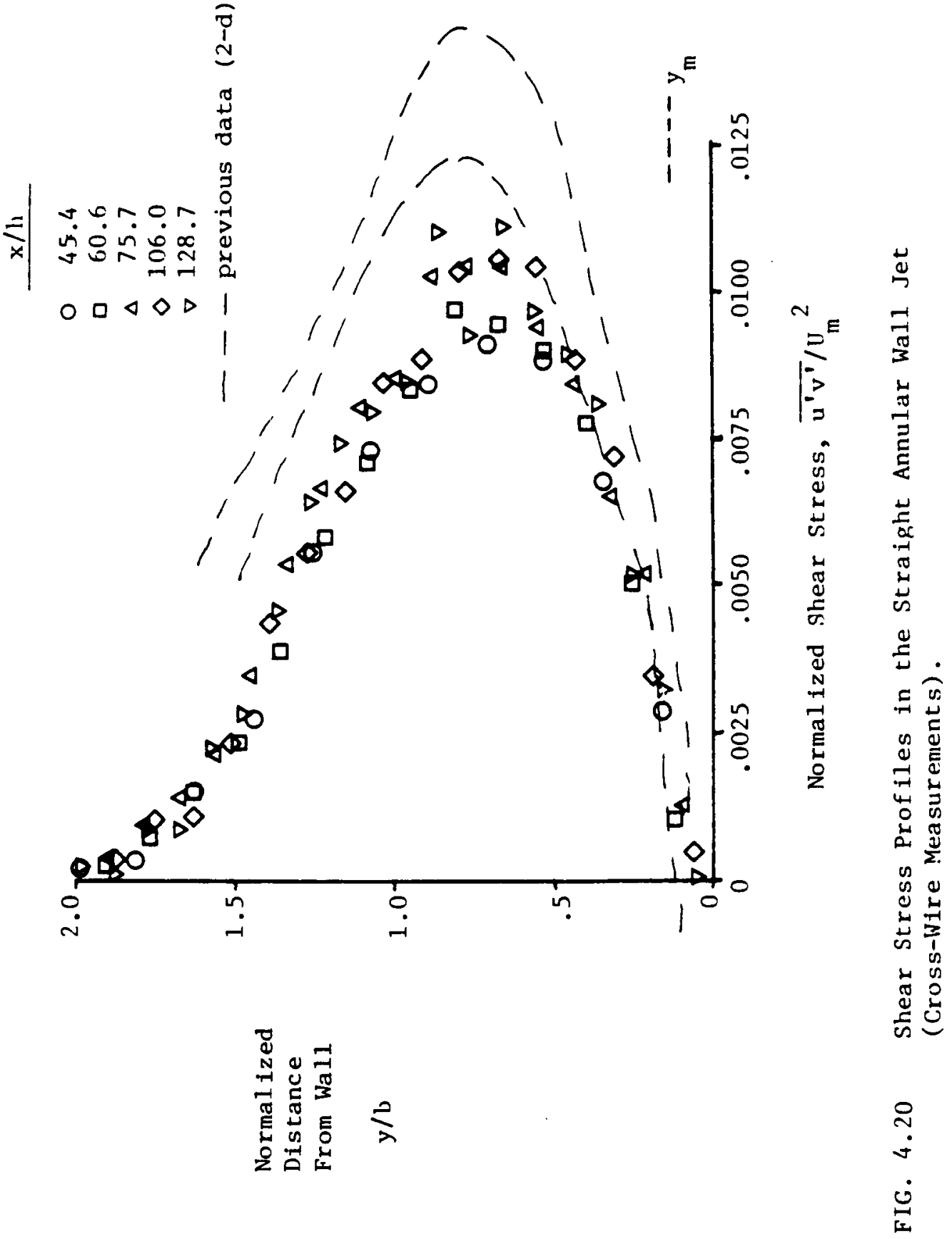




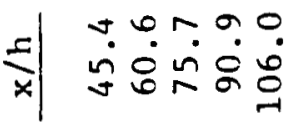
$O \triangle \triangle \triangle D$

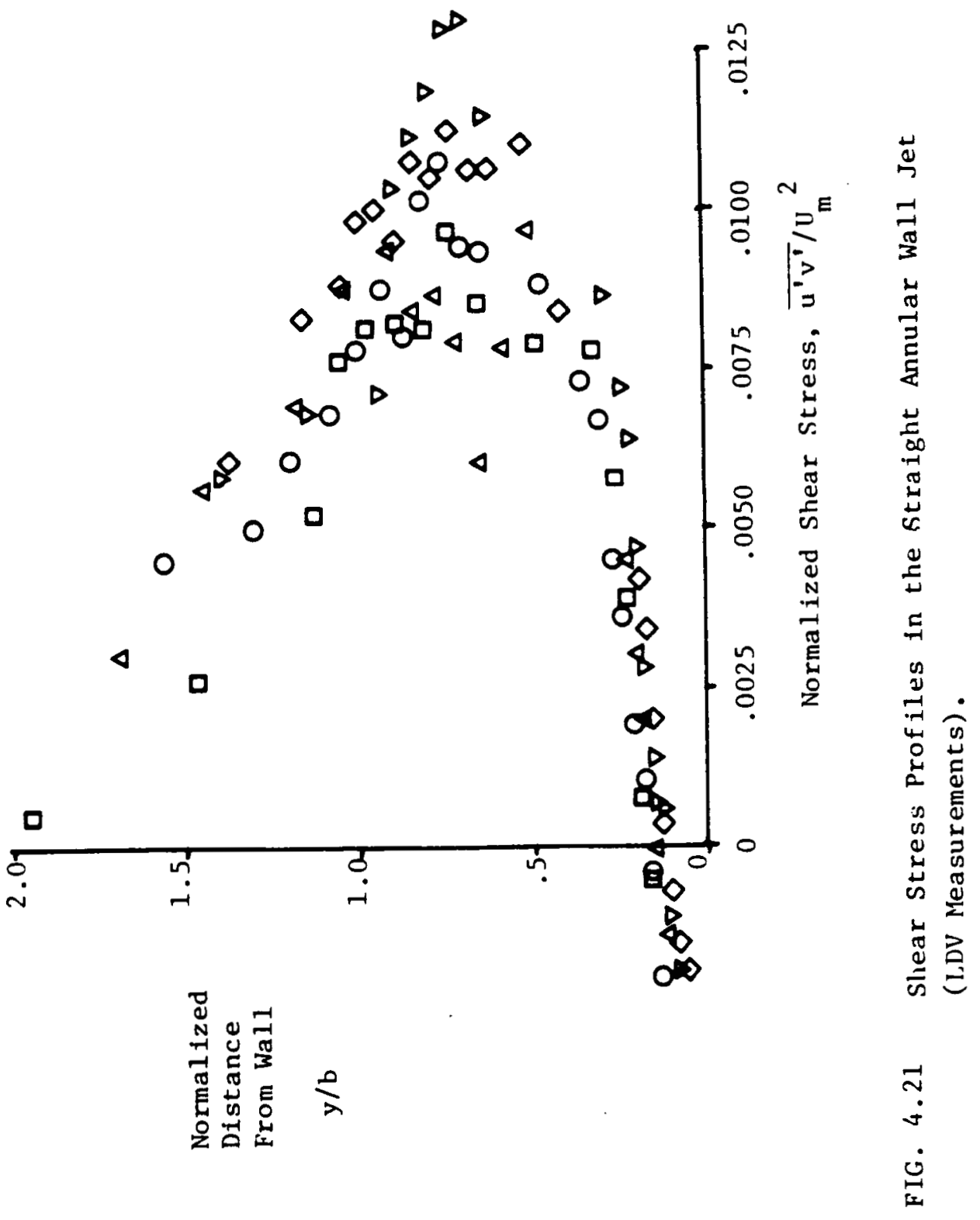




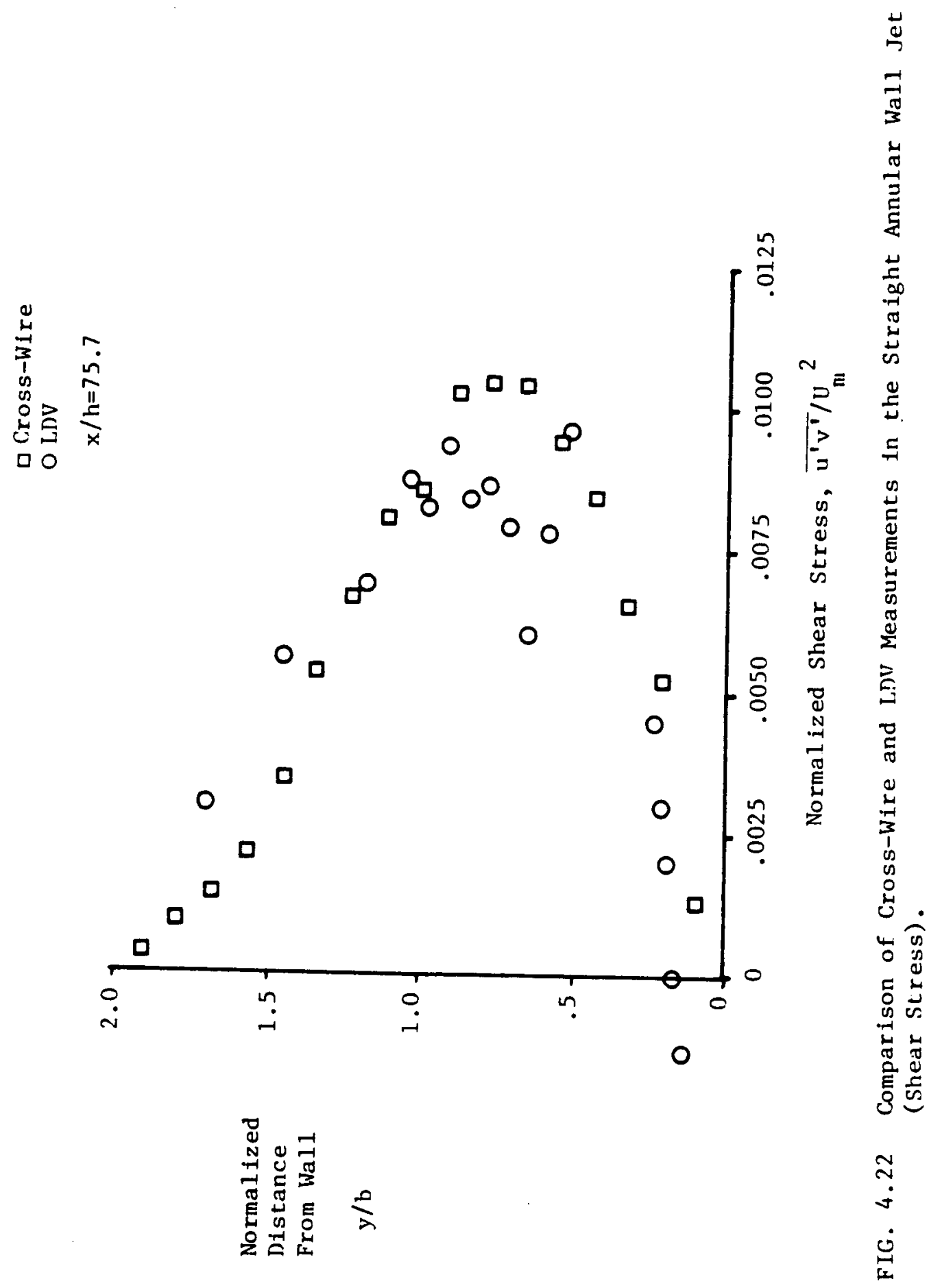




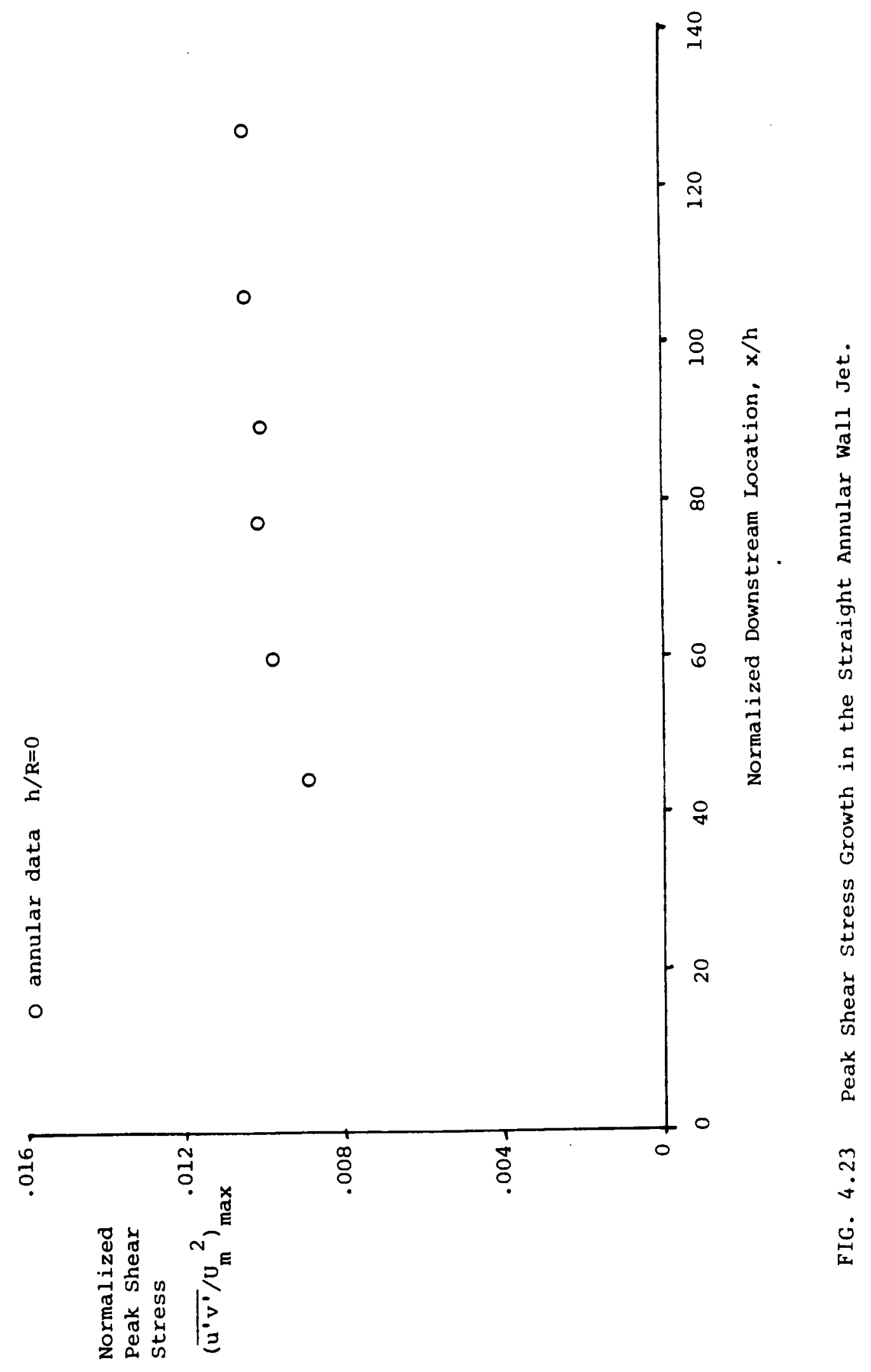




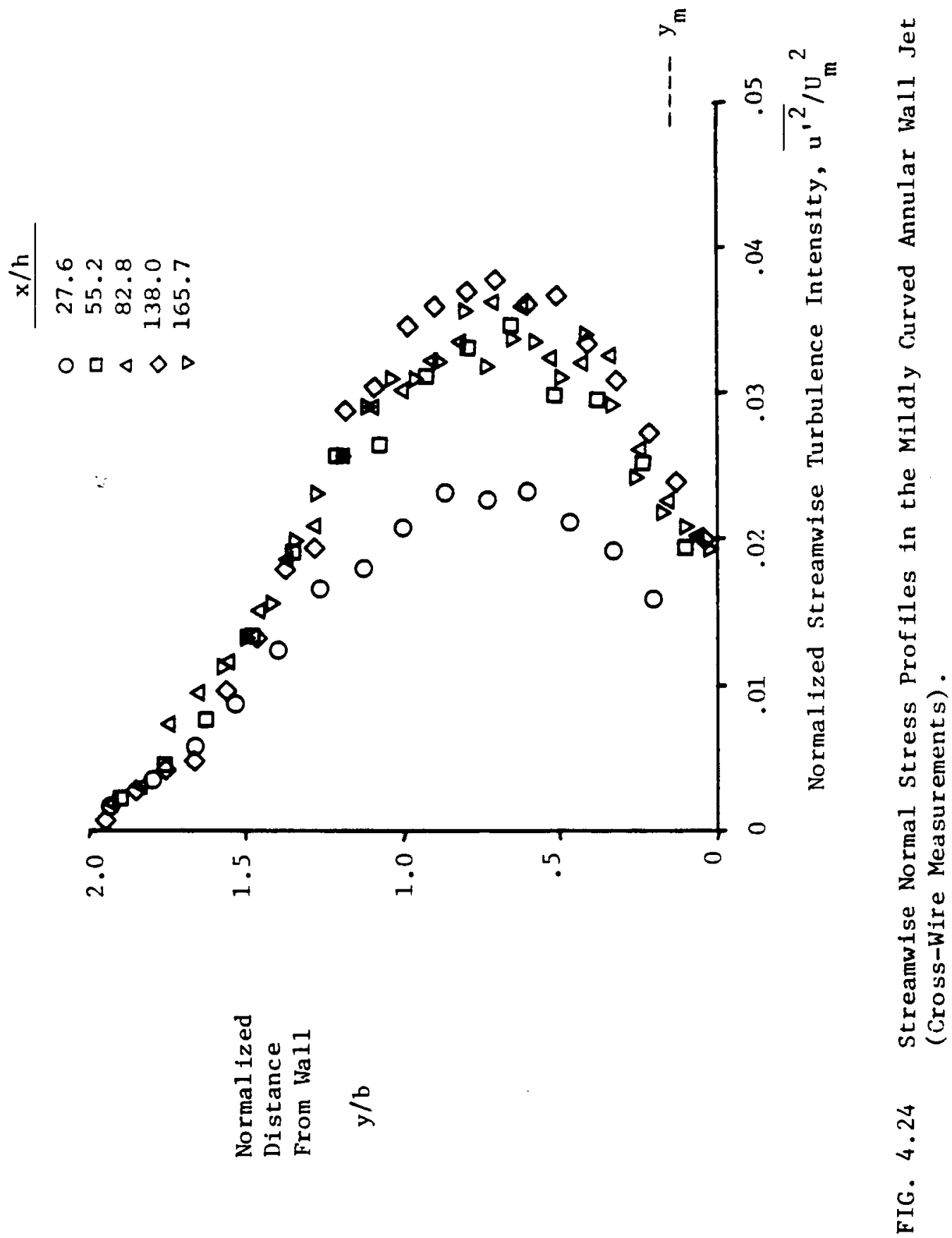




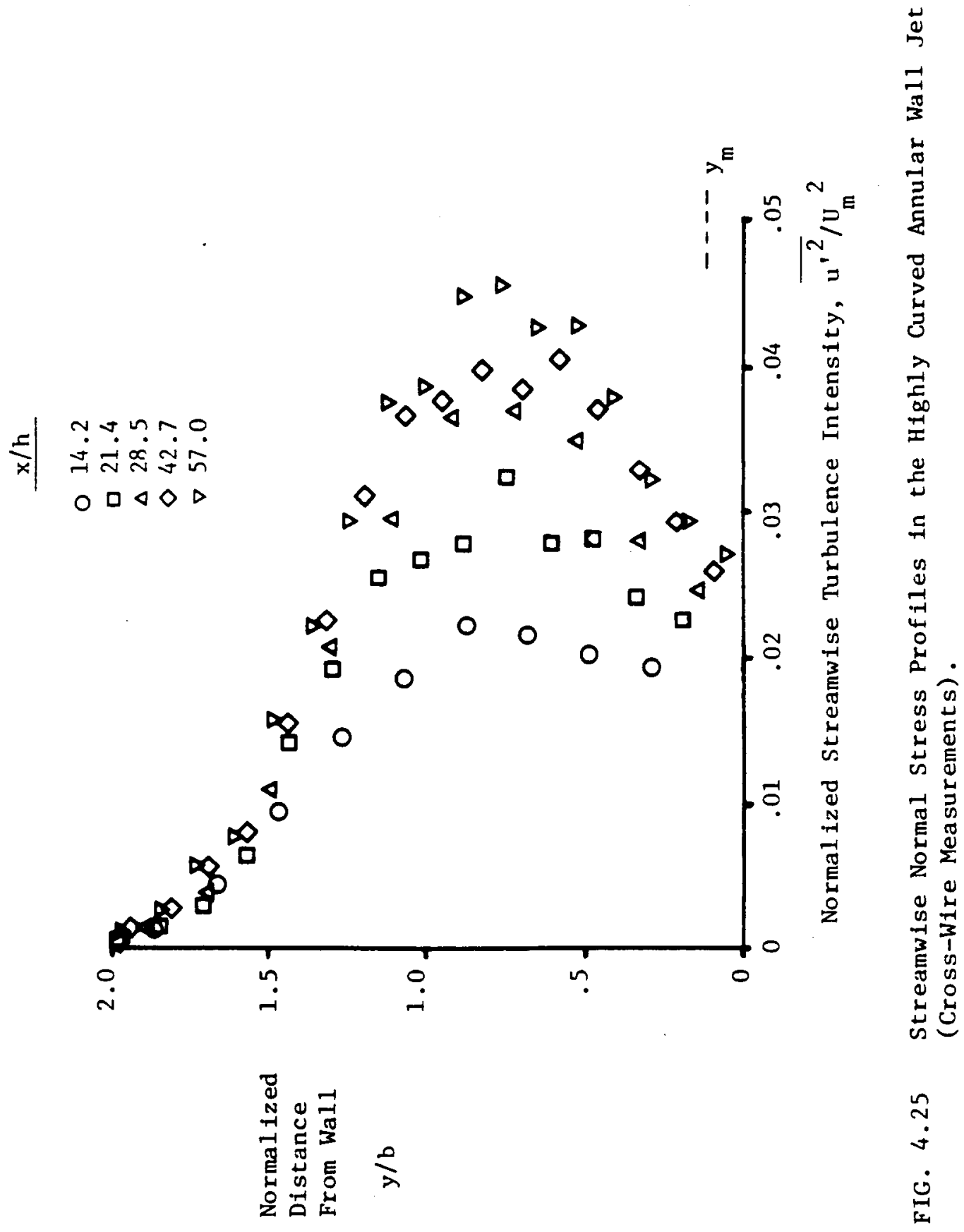



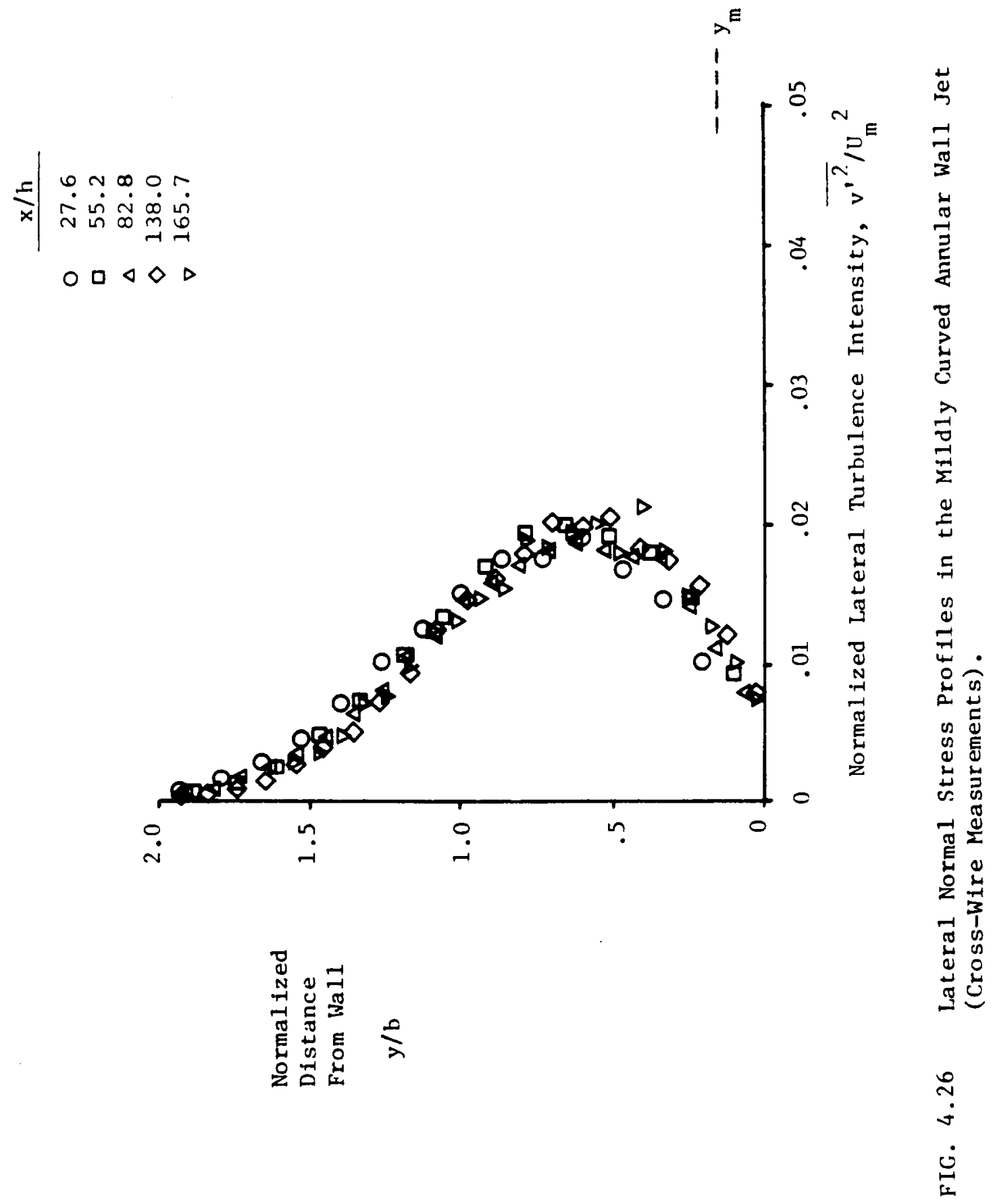


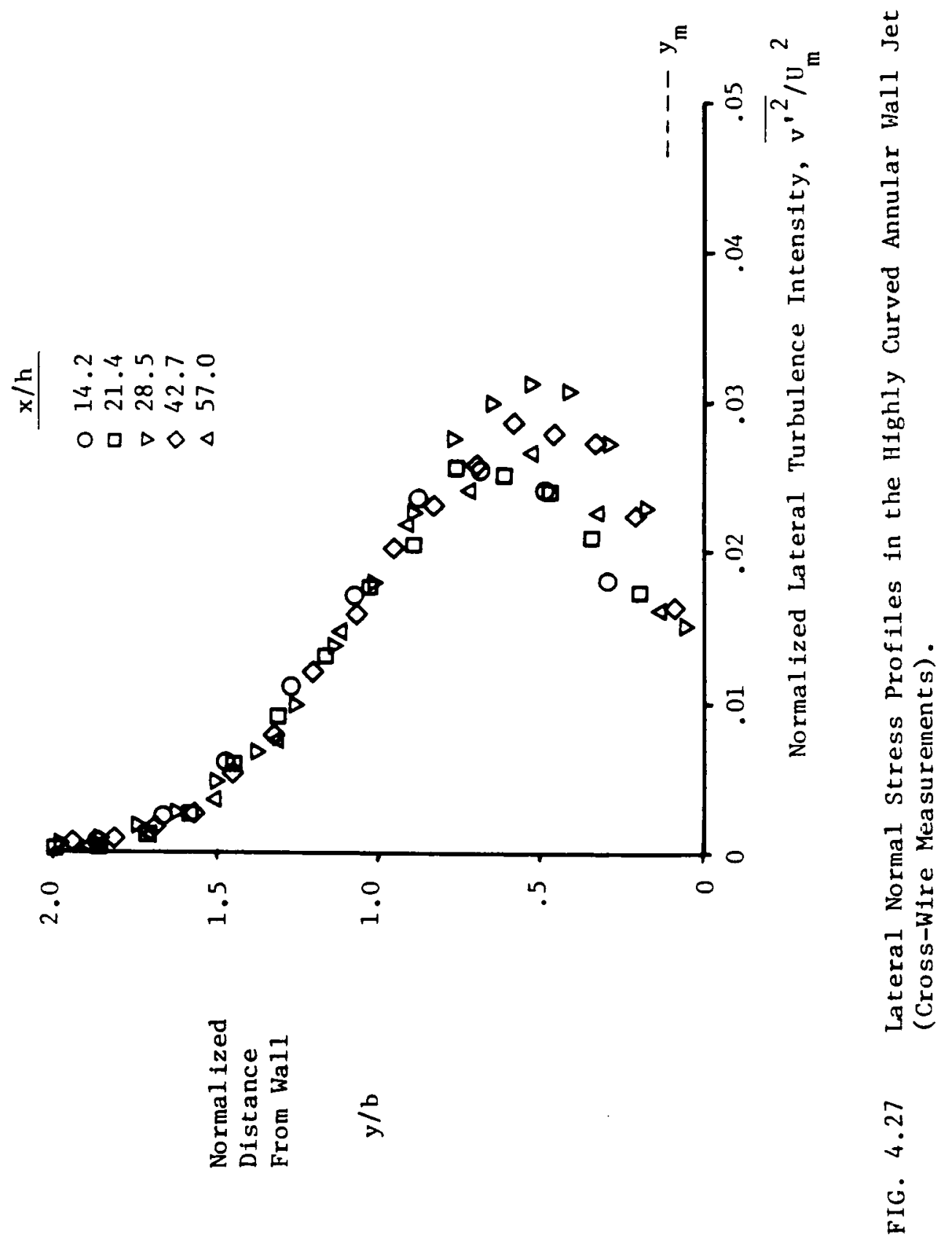



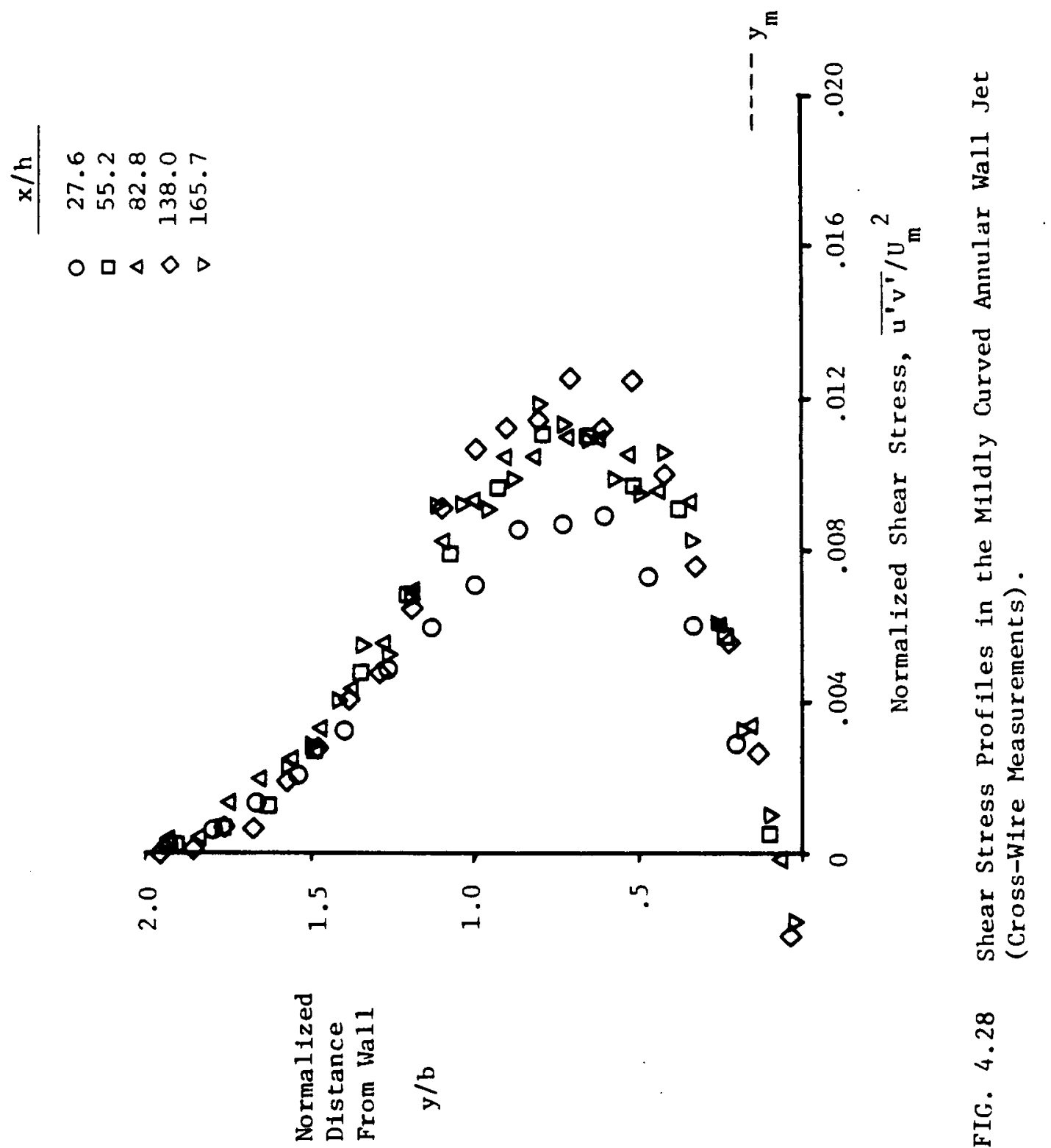

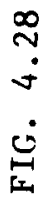




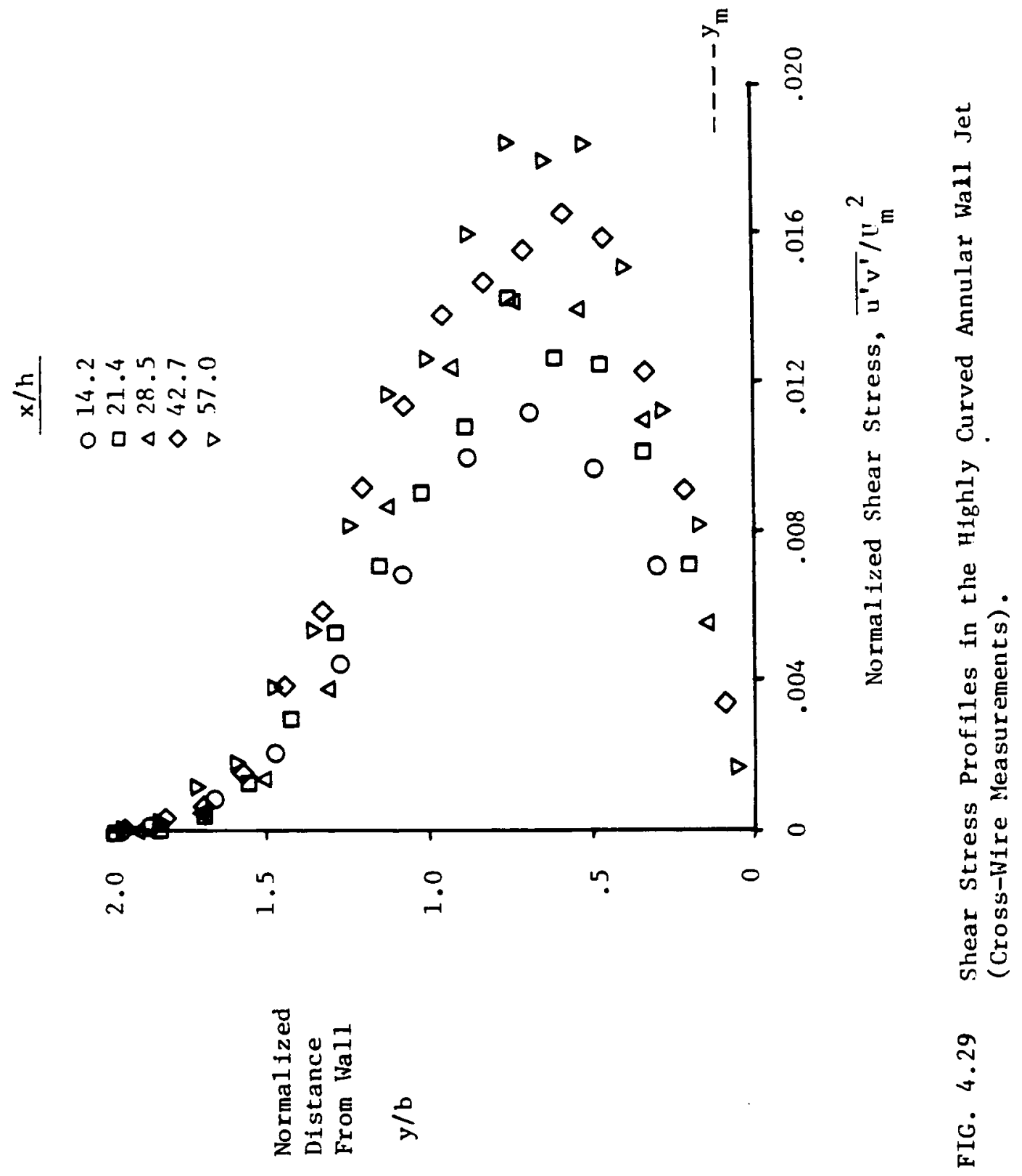




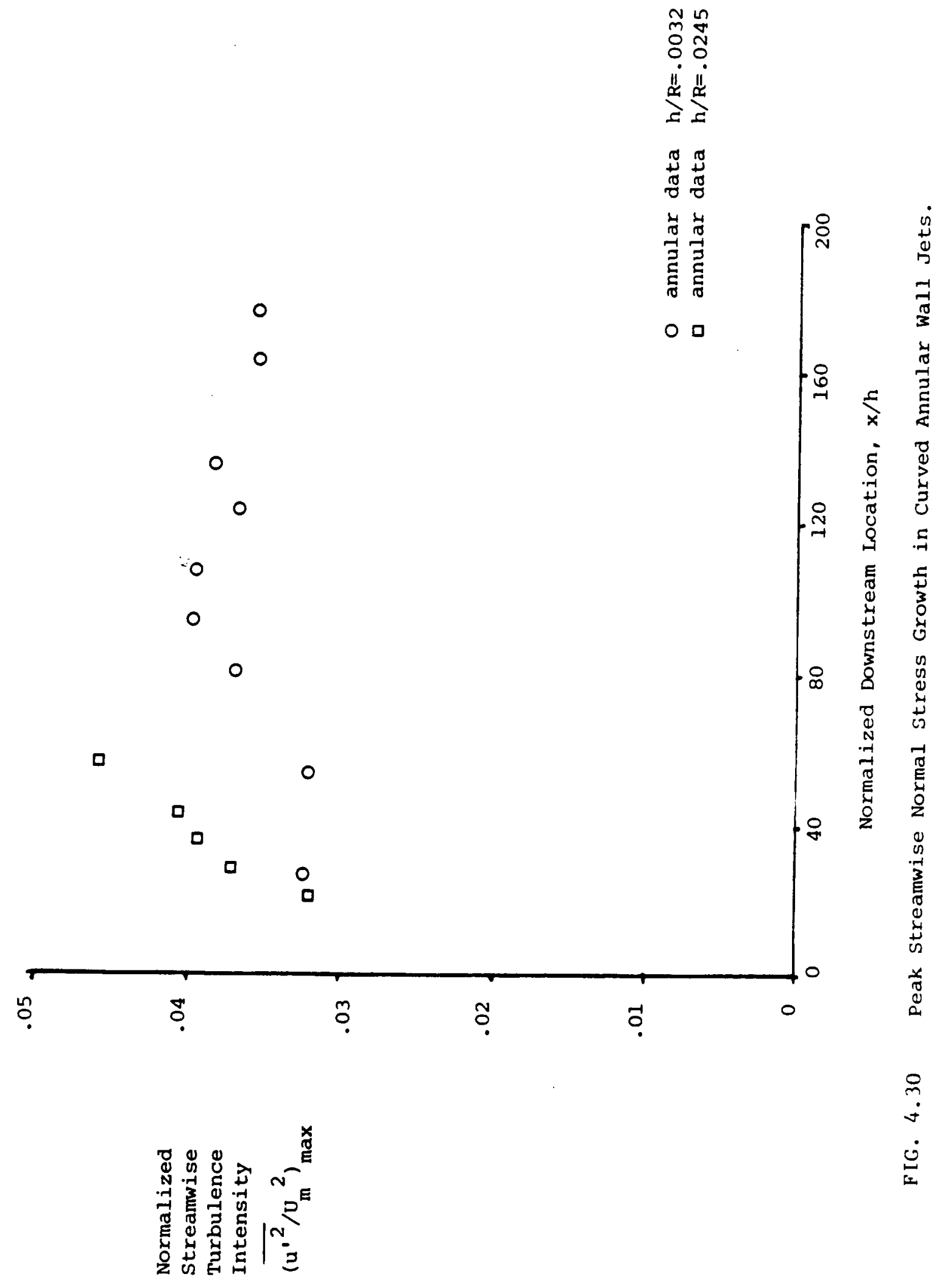




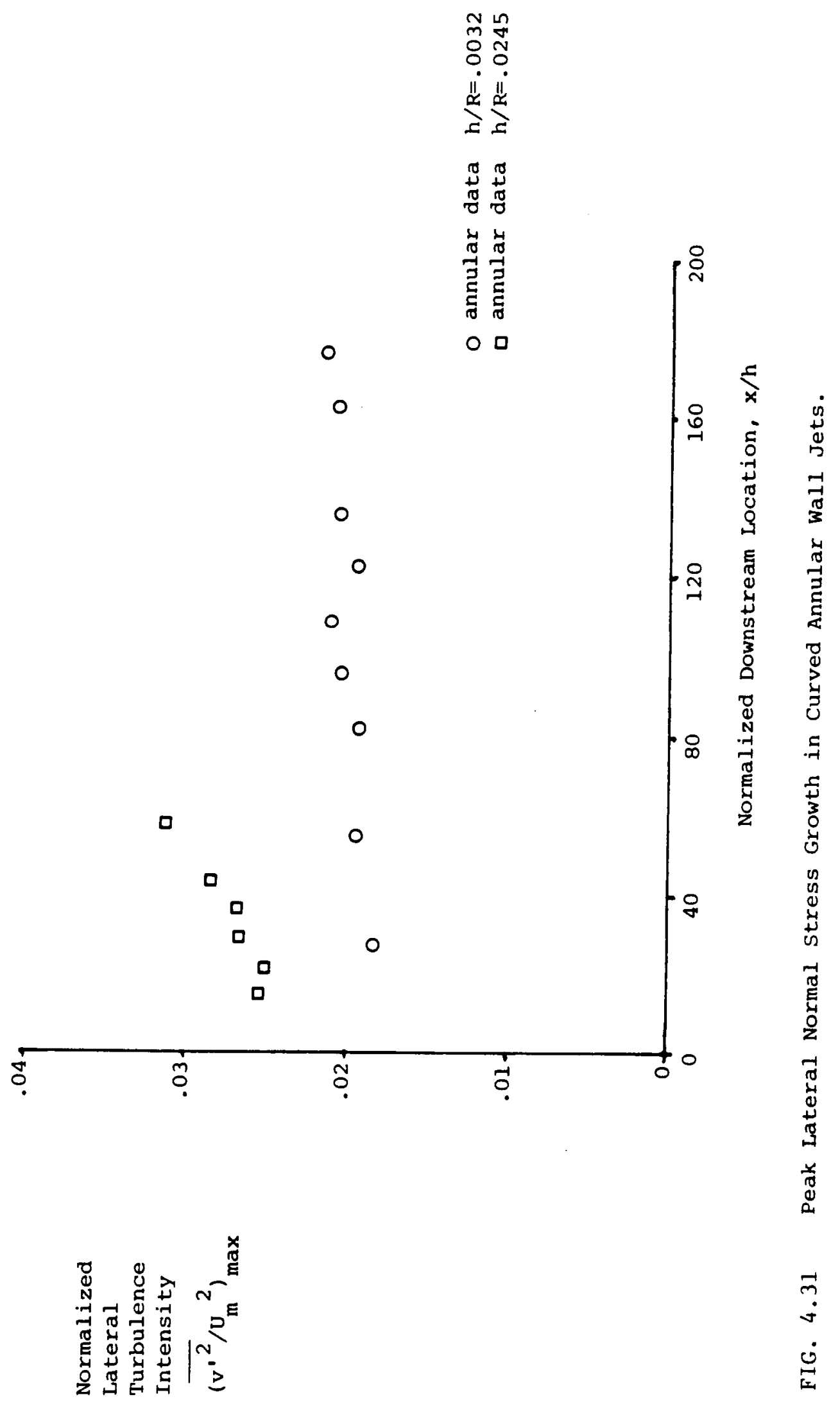




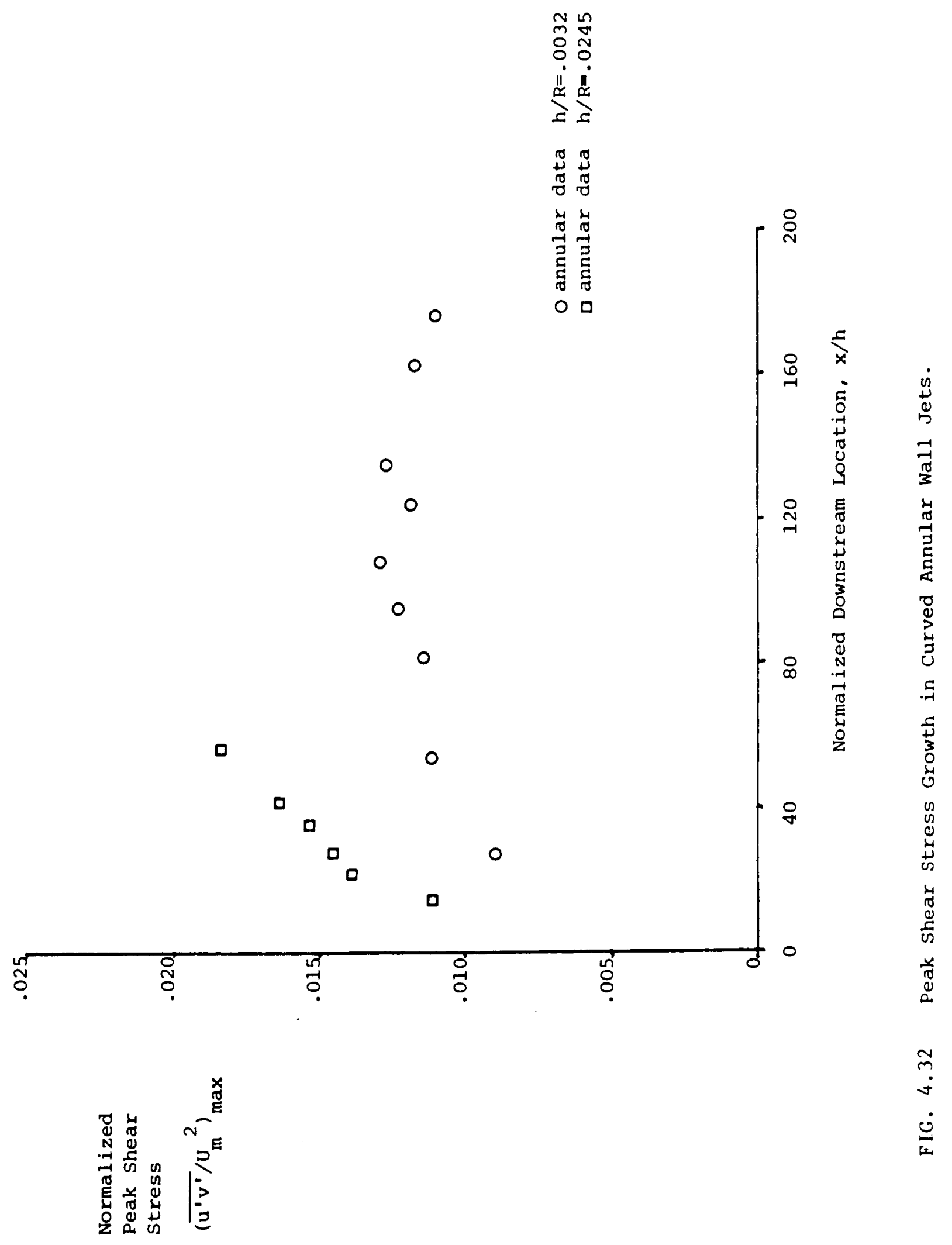




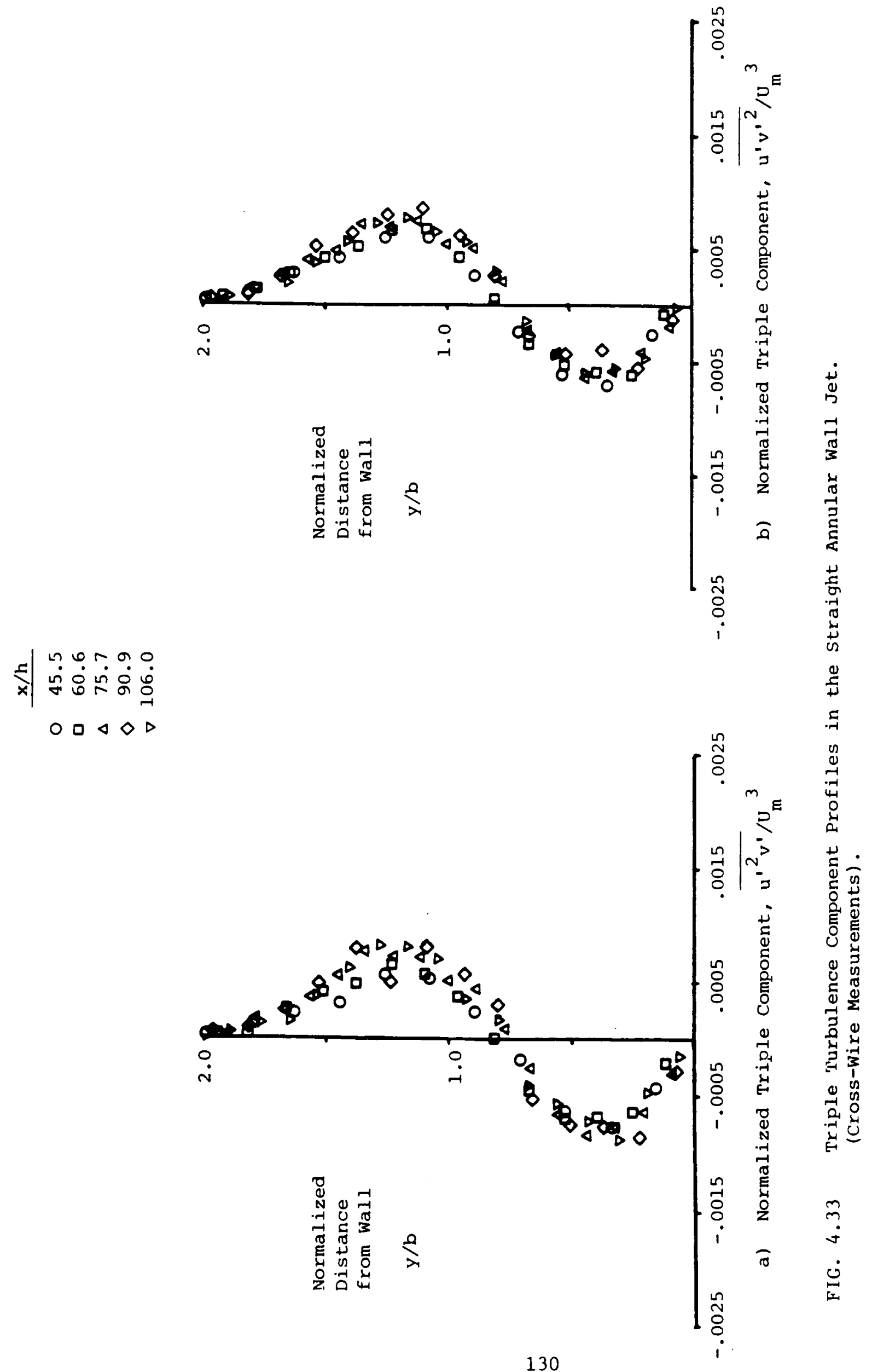




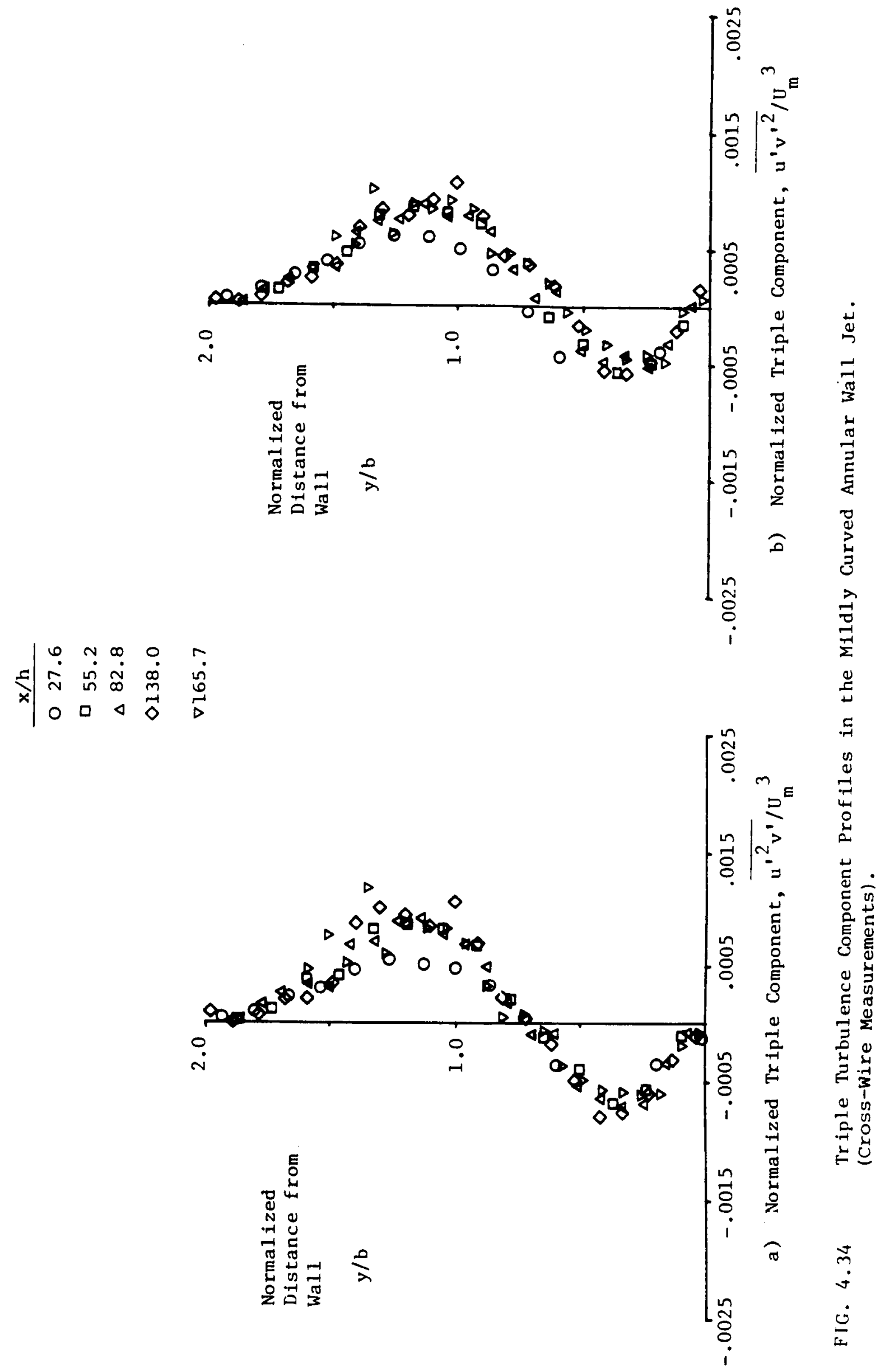




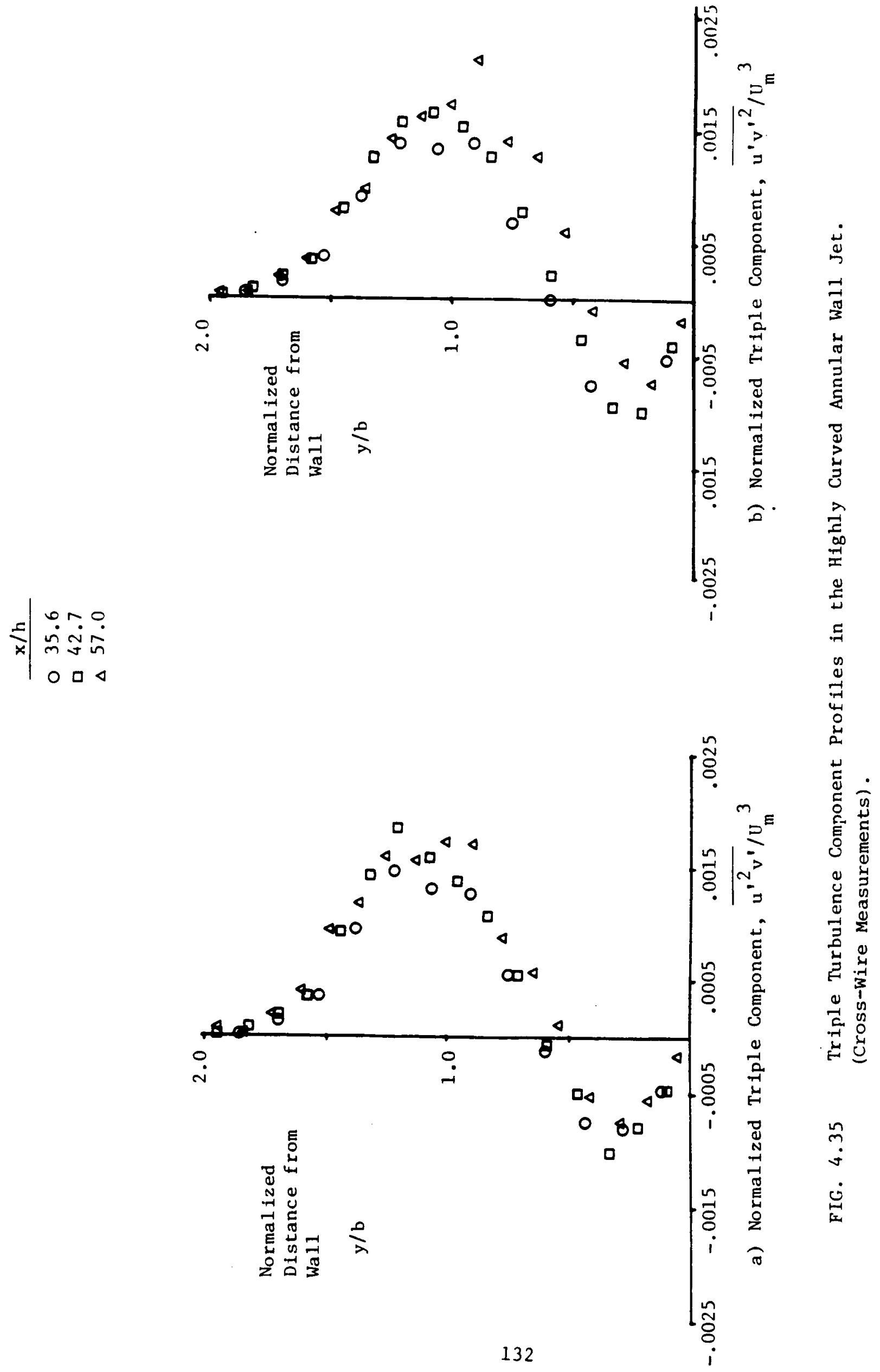




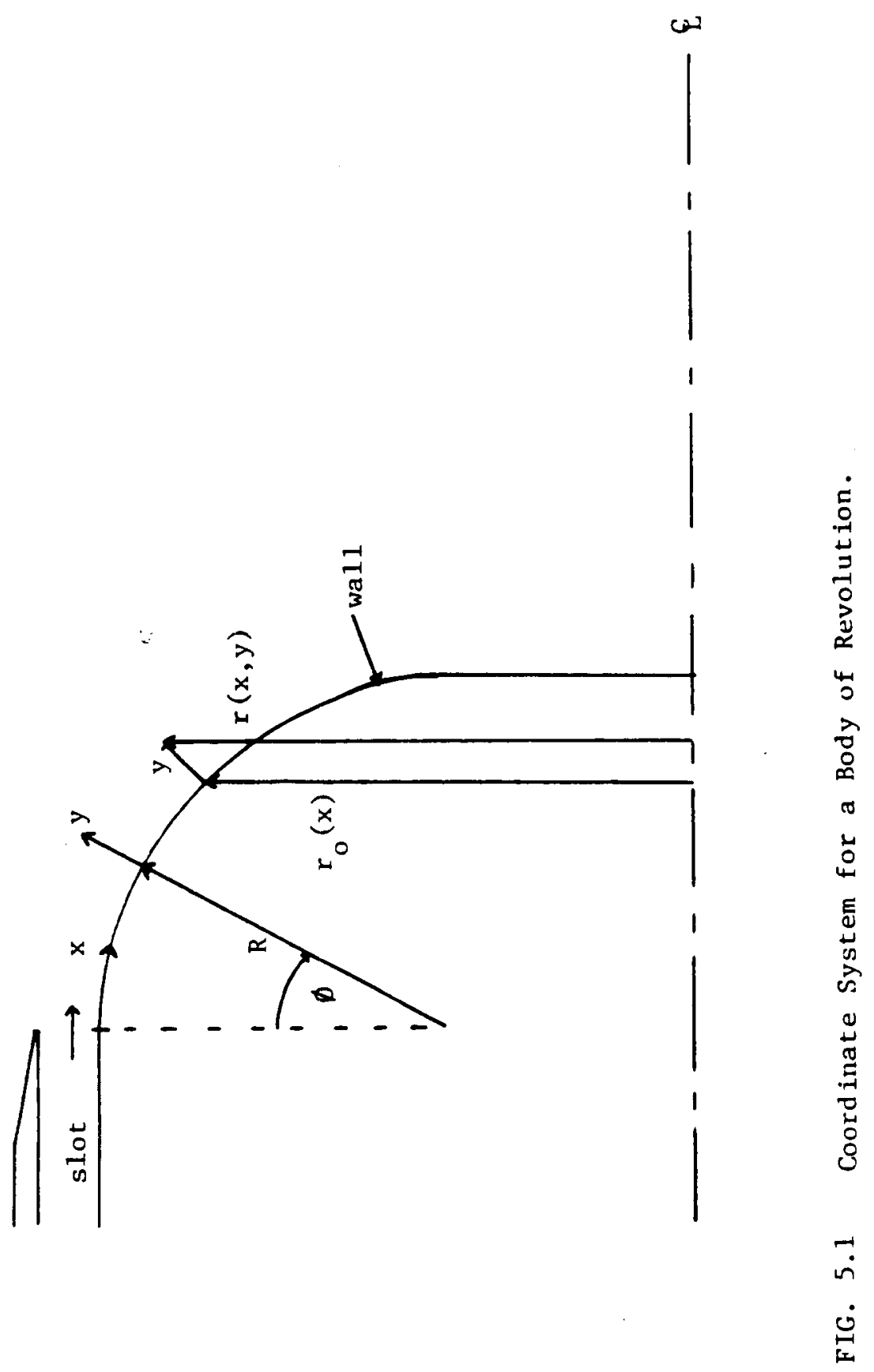




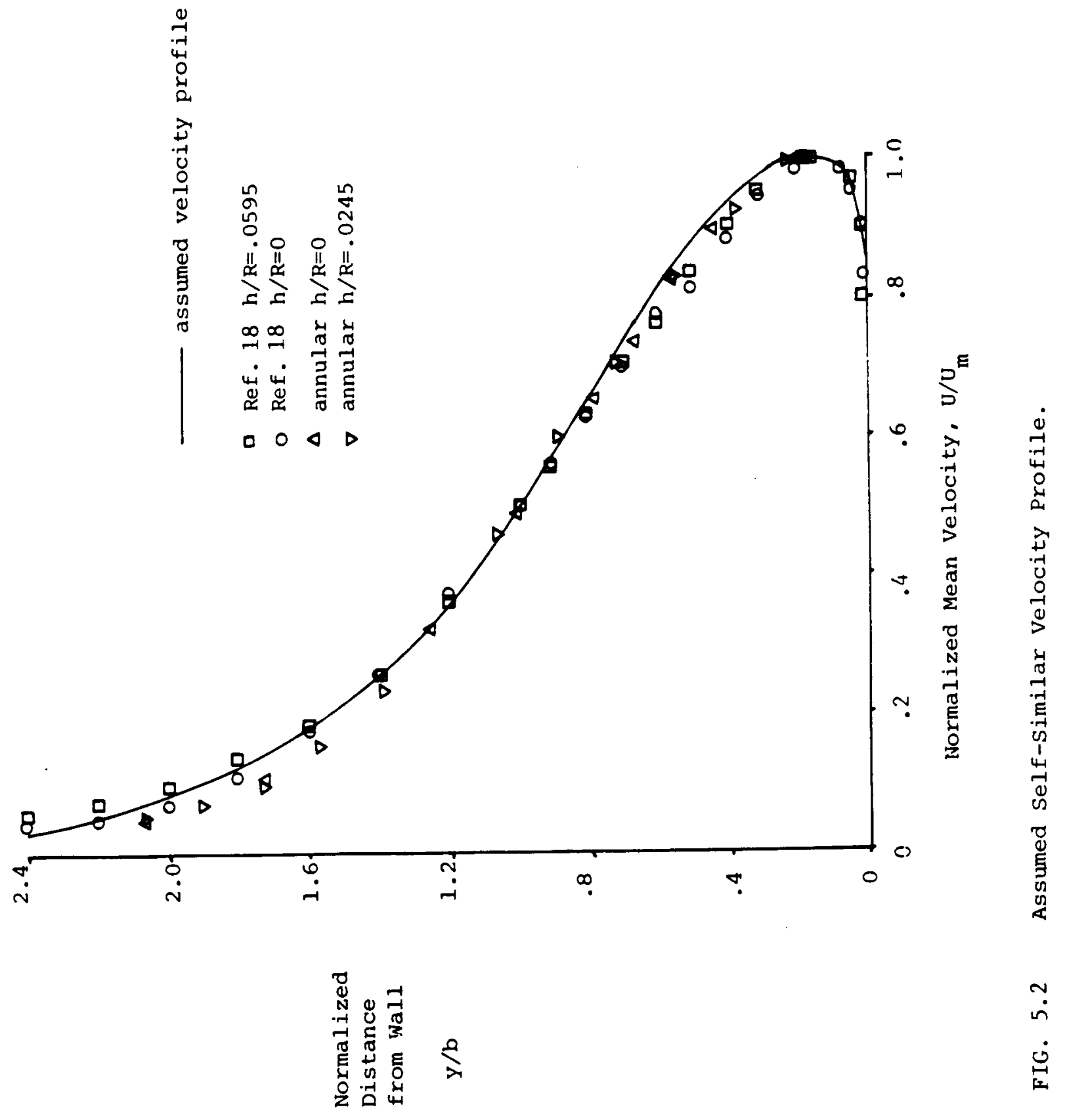




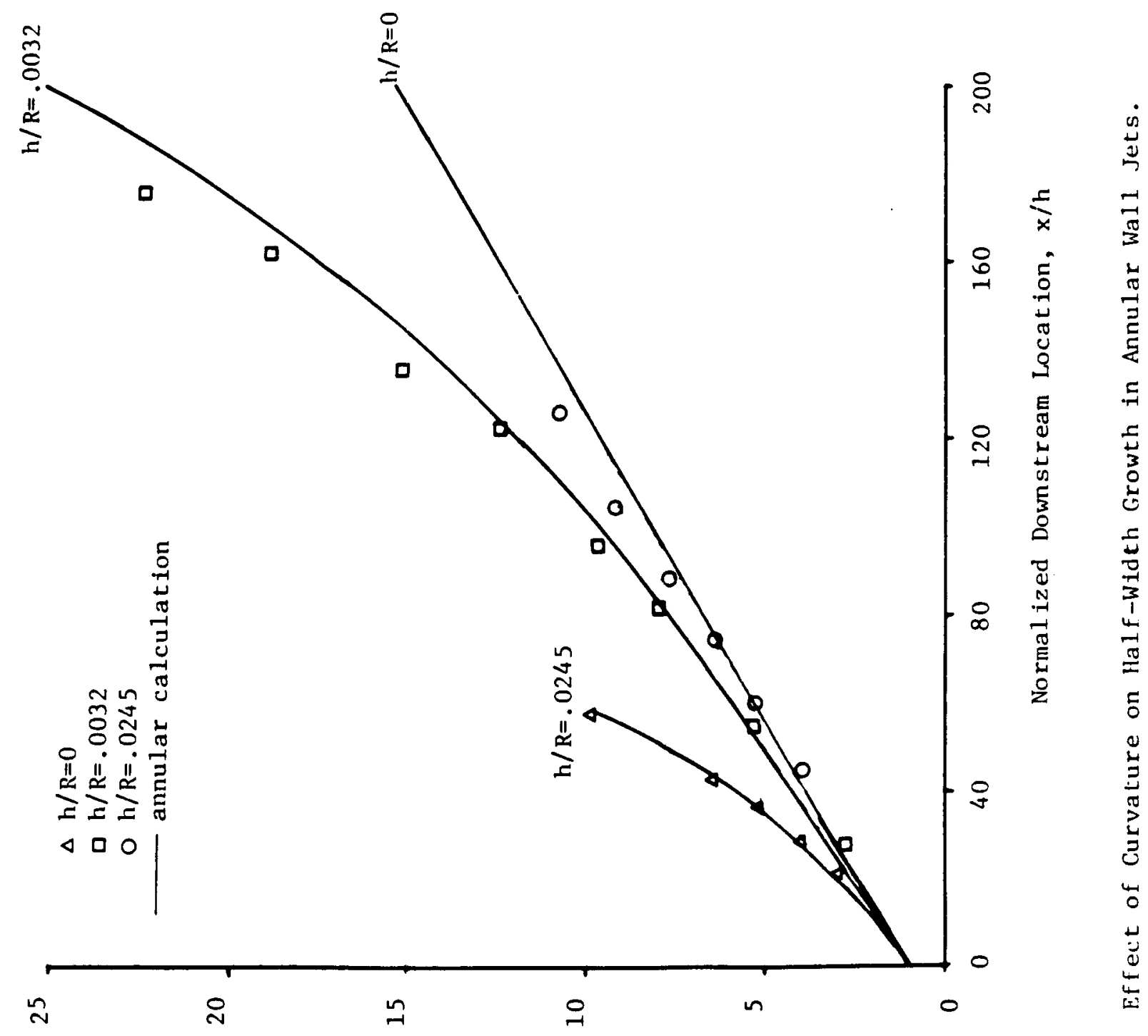

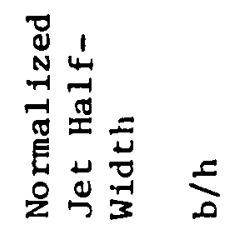

$\dot{m}$ 


$$
\begin{aligned}
& \stackrel{\infty}{\rightarrow} \rightarrow \rightarrow \stackrel{\infty}{\rightarrow}
\end{aligned}
$$

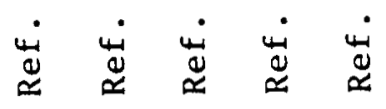

$$
\begin{aligned}
& \triangle \diamond \triangleleft \square 0
\end{aligned}
$$

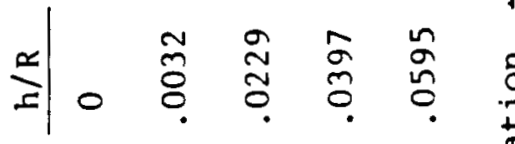

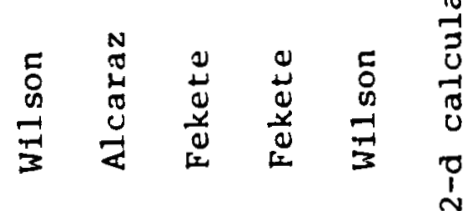

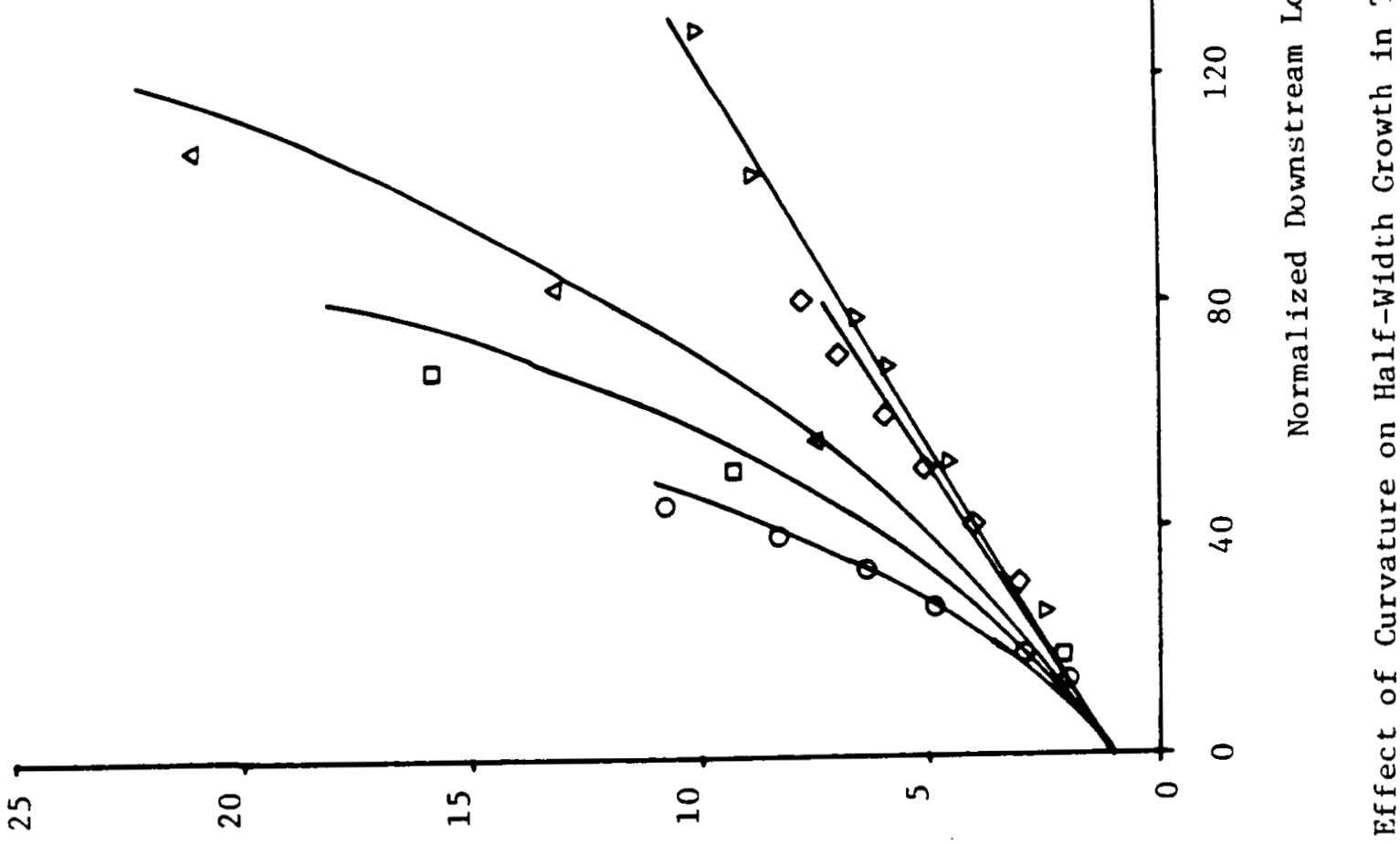

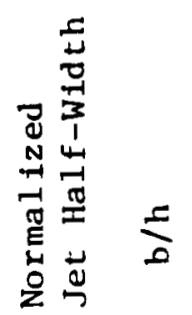

$\stackrel{v}{\dot{0}}$ 


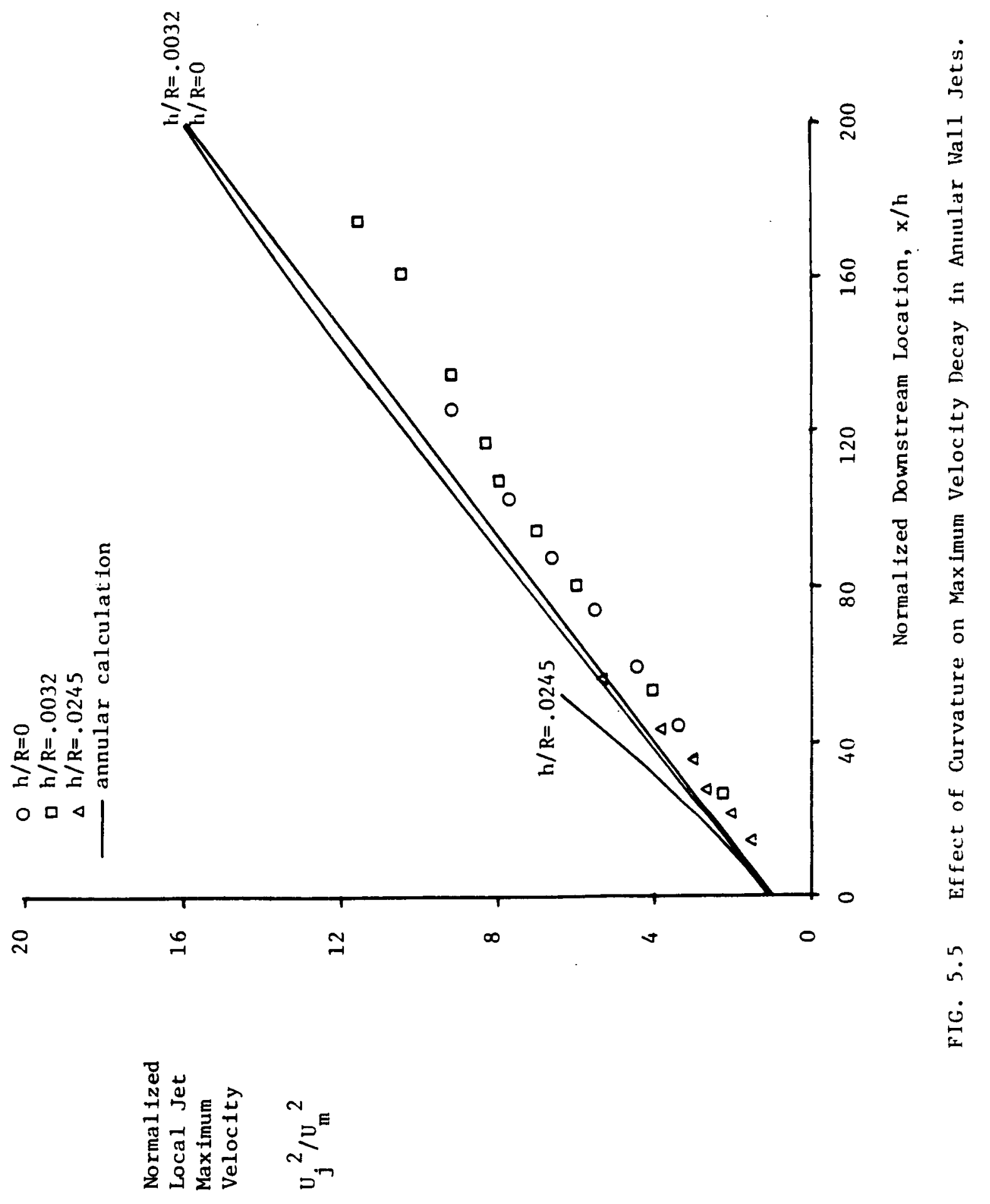




$$
\begin{aligned}
& \stackrel{\infty}{\rightarrow} \rightarrow \infty \\
& \text { 岁 } \\
& 0<\square \quad 0
\end{aligned}
$$

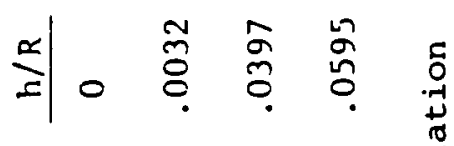

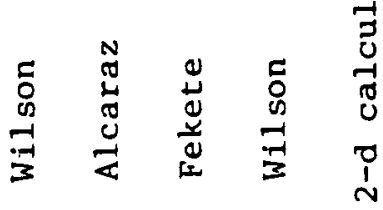

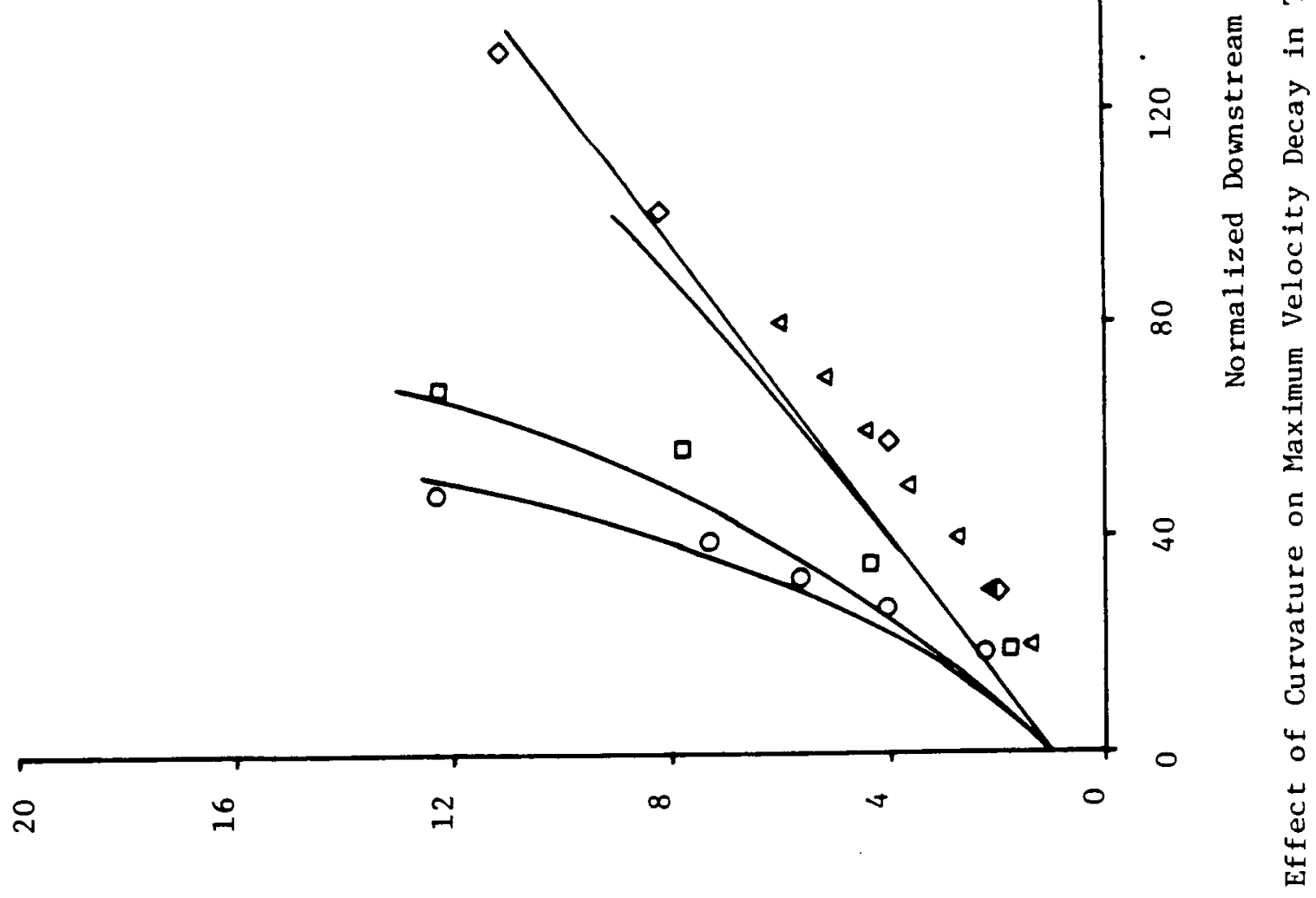

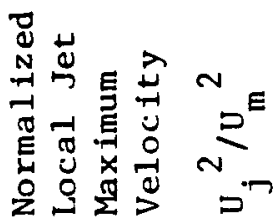

$\dot{0}$ 


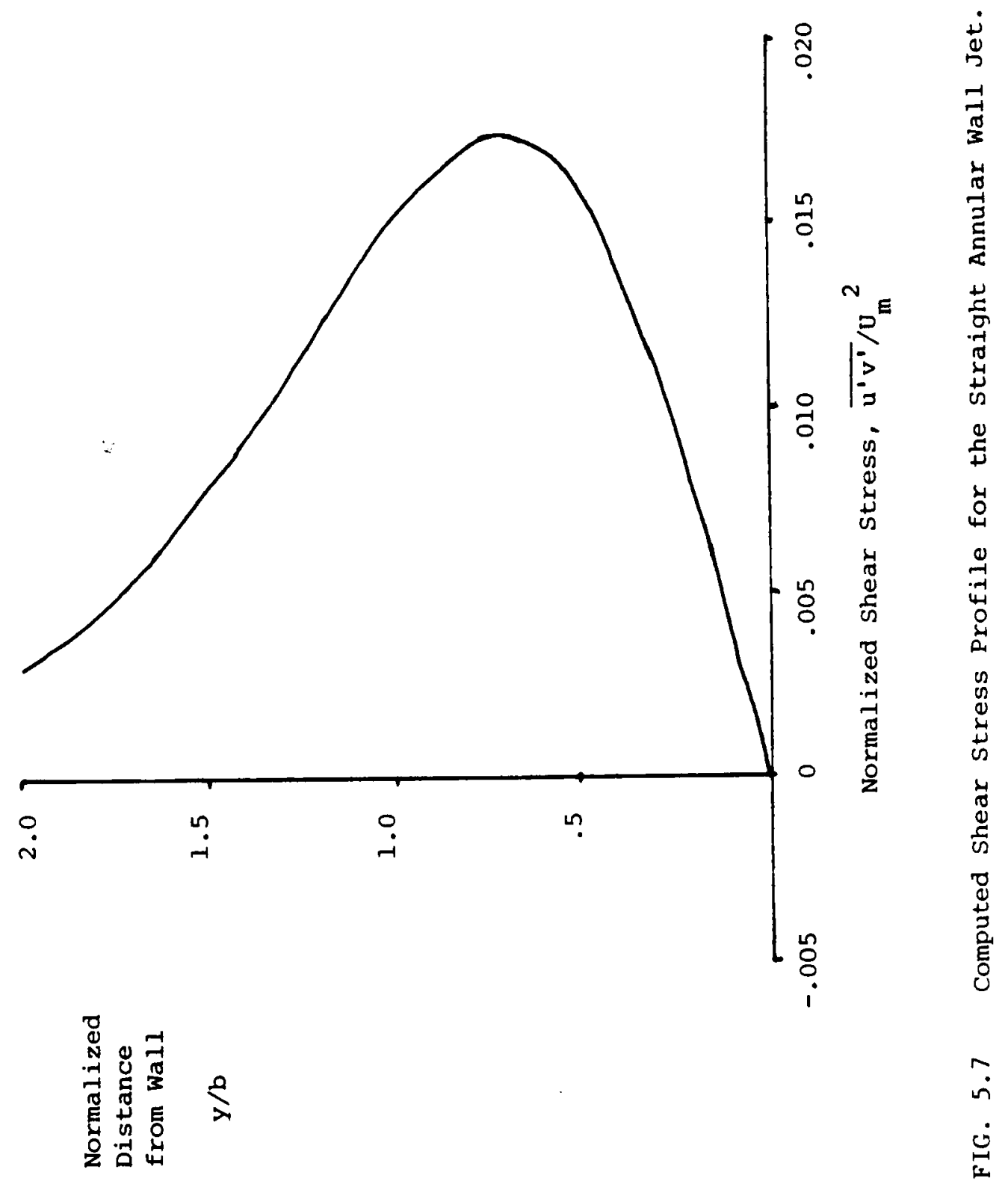




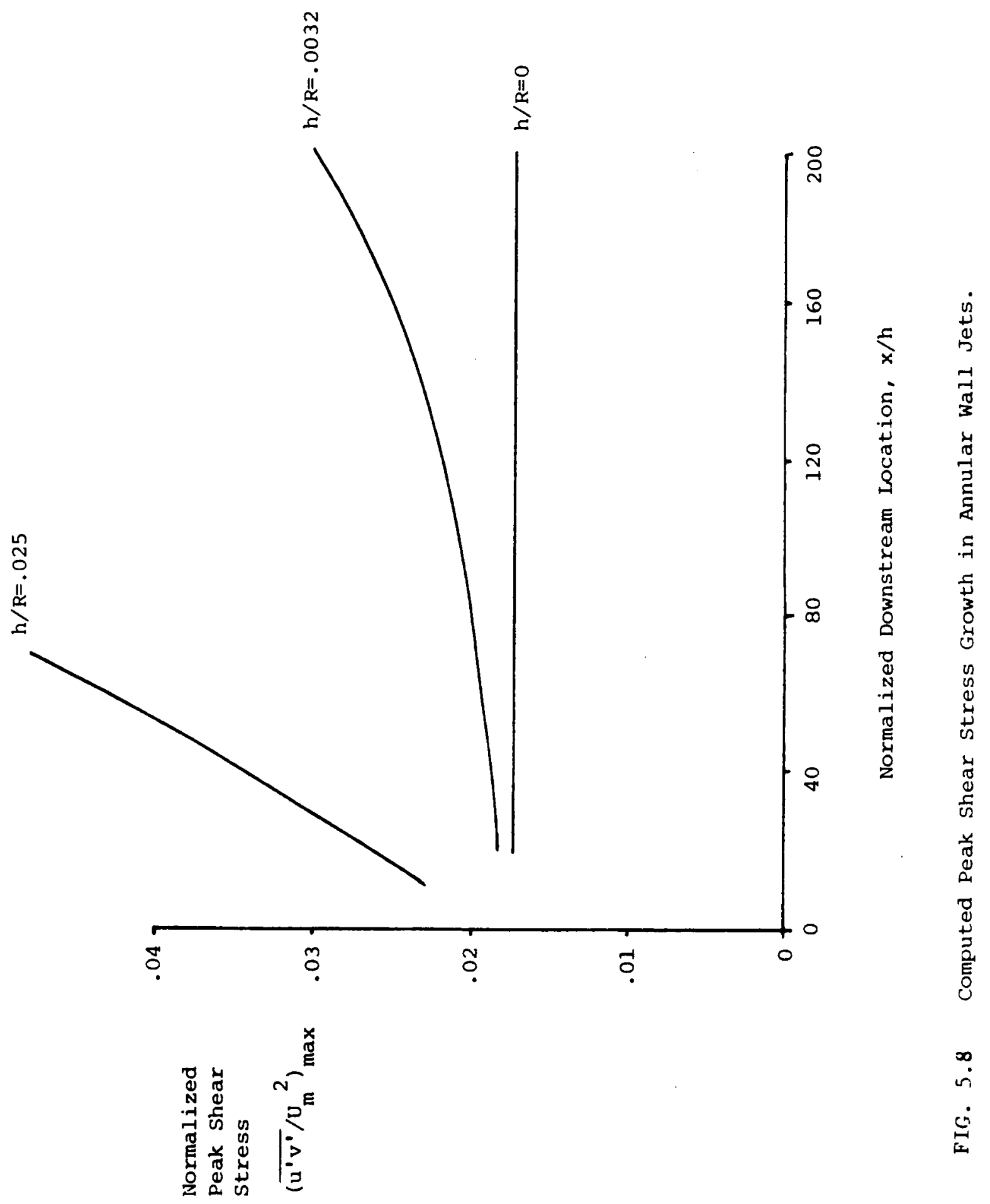




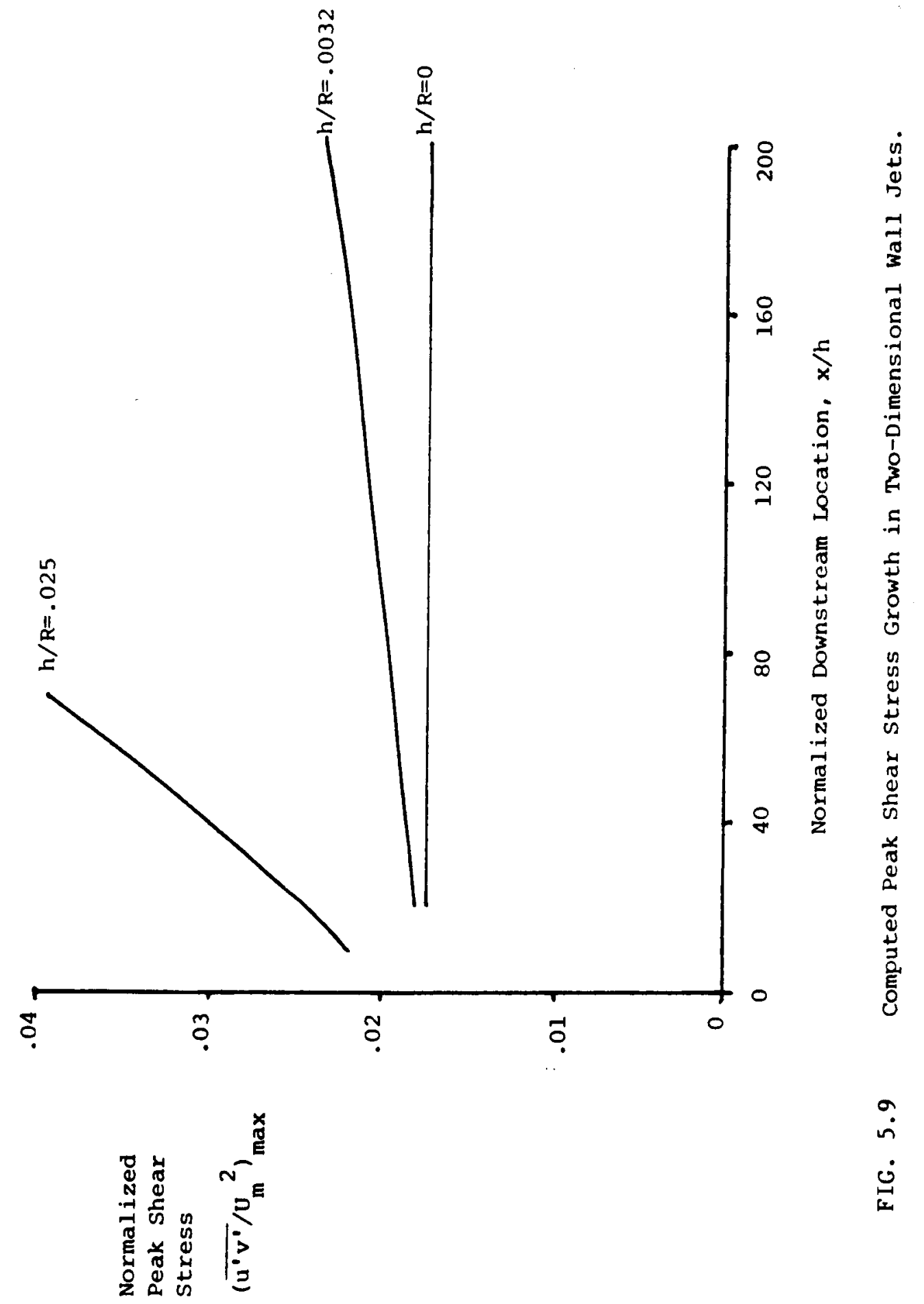




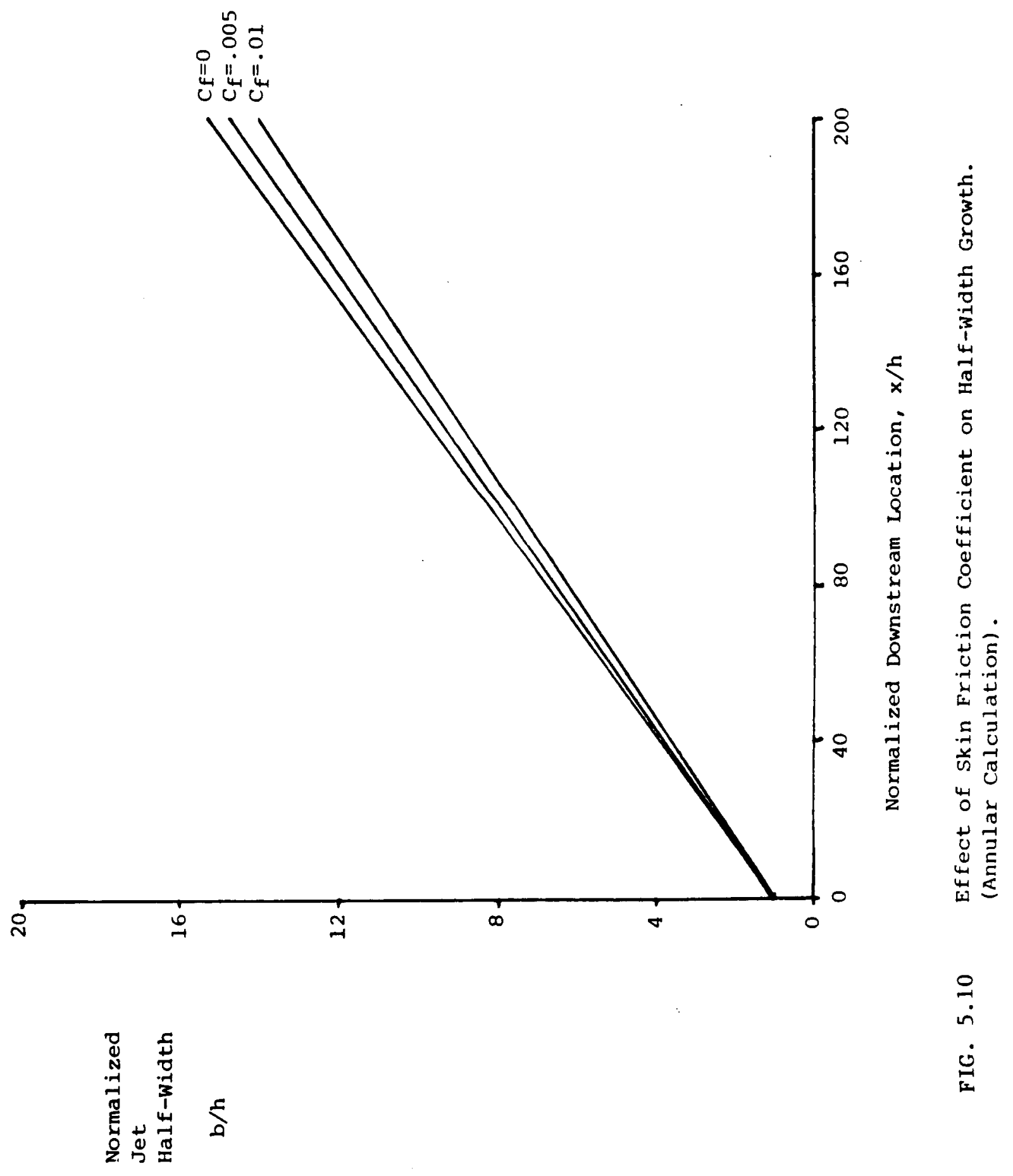




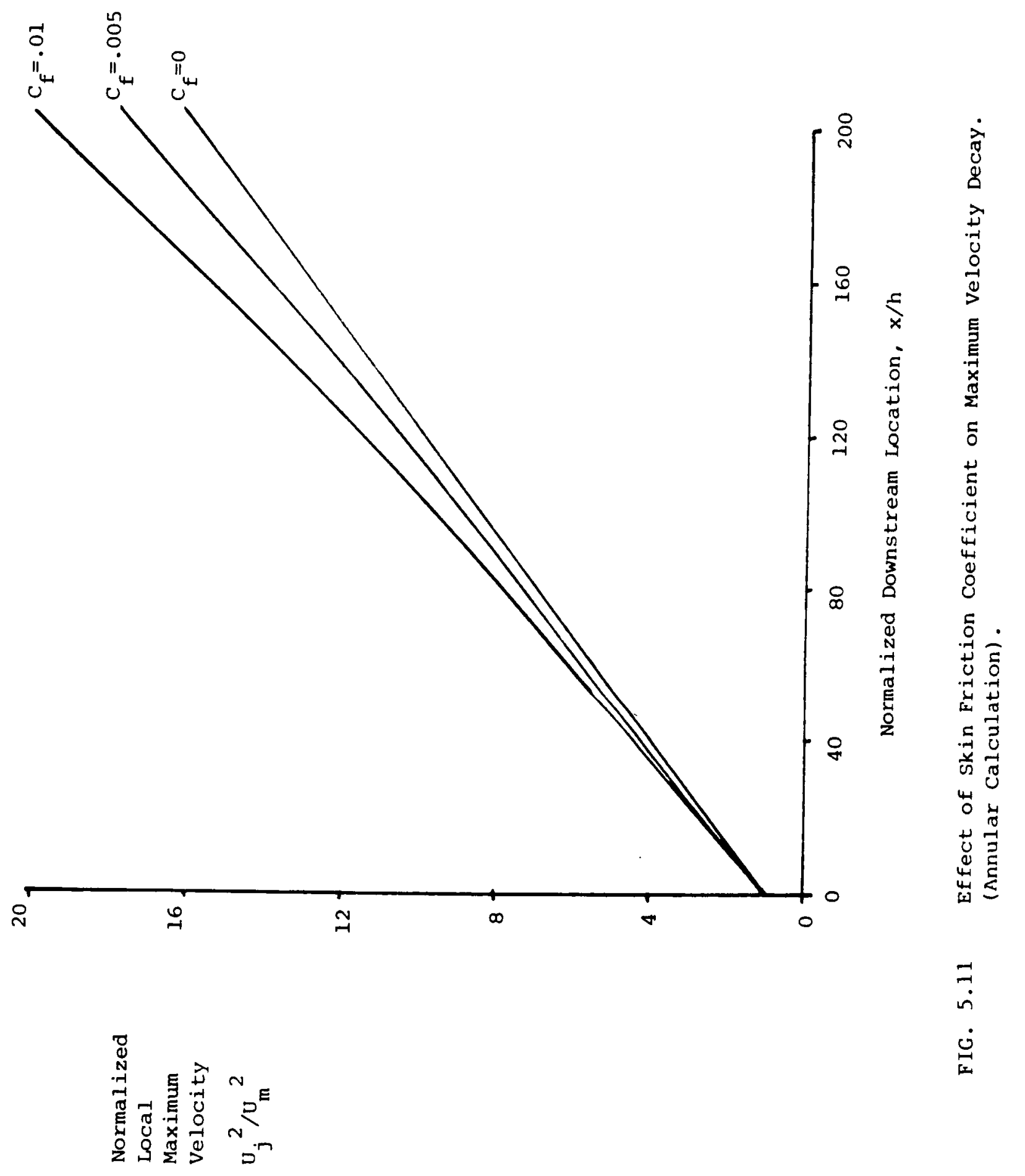


$\mid \begin{array}{lll}1 & 1 \\ 1 & 1 \\ 1 & 1 \\ 1 & 1 \\ 2 & 1 \\ 0 & 0 & 0 \\ 0 & 0\end{array}$

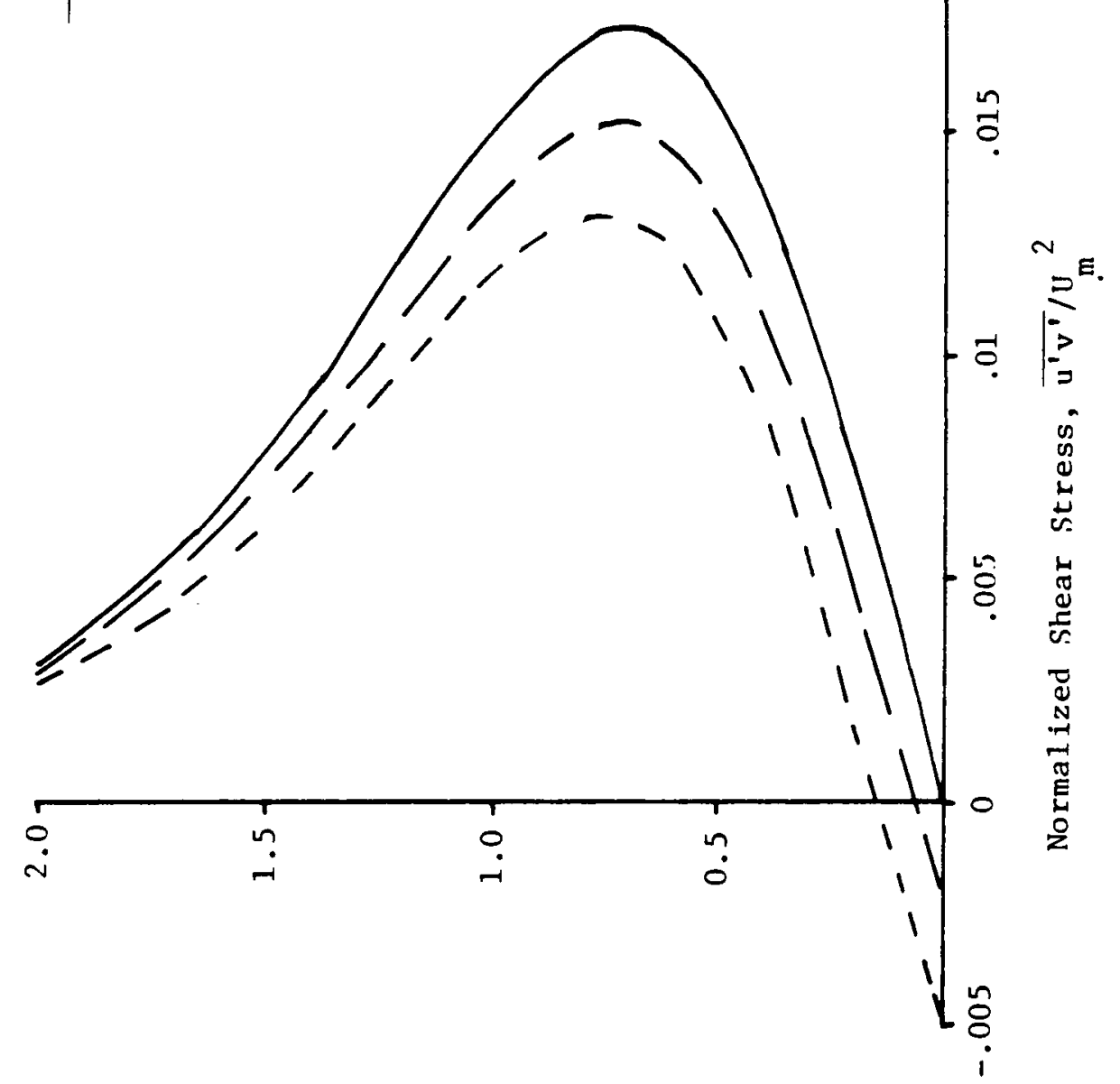

站

i

곡 극

$\Rightarrow \frac{\pi}{3}$

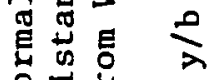

员䓃点

$\stackrel{\Xi}{0}$

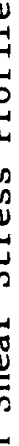

동

岂

苗

$\frac{5}{0}$

4

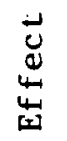

in

这 


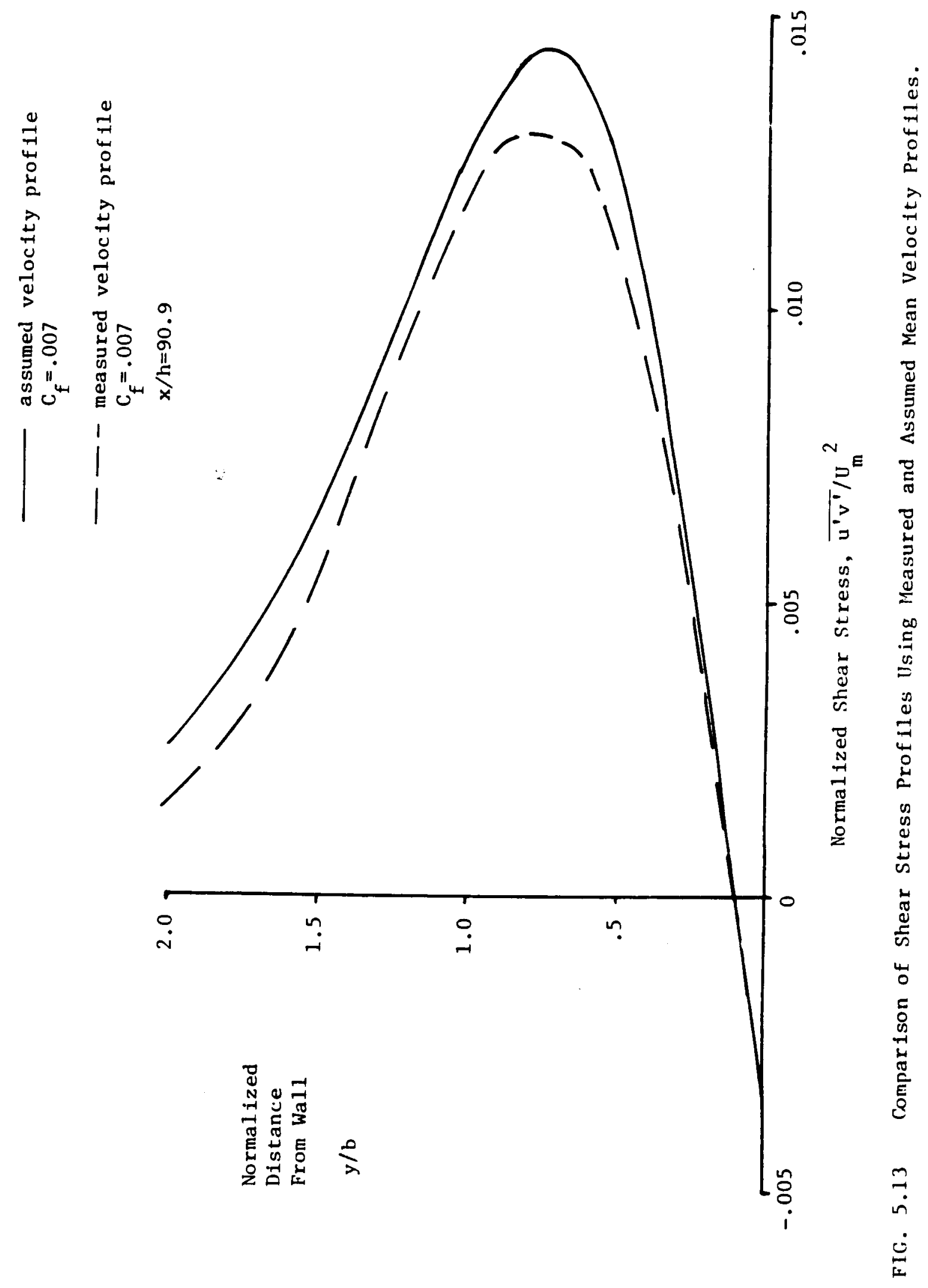




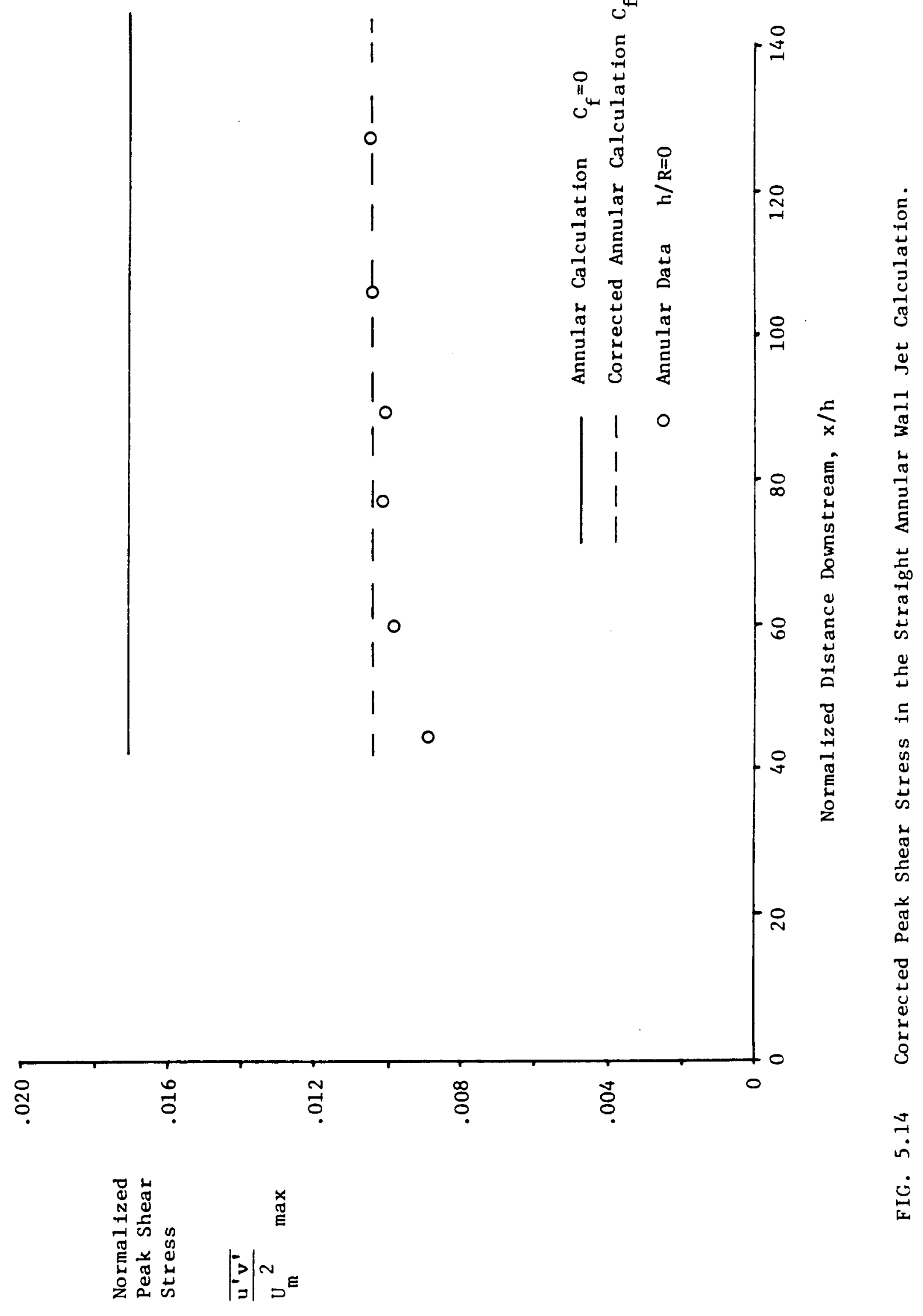




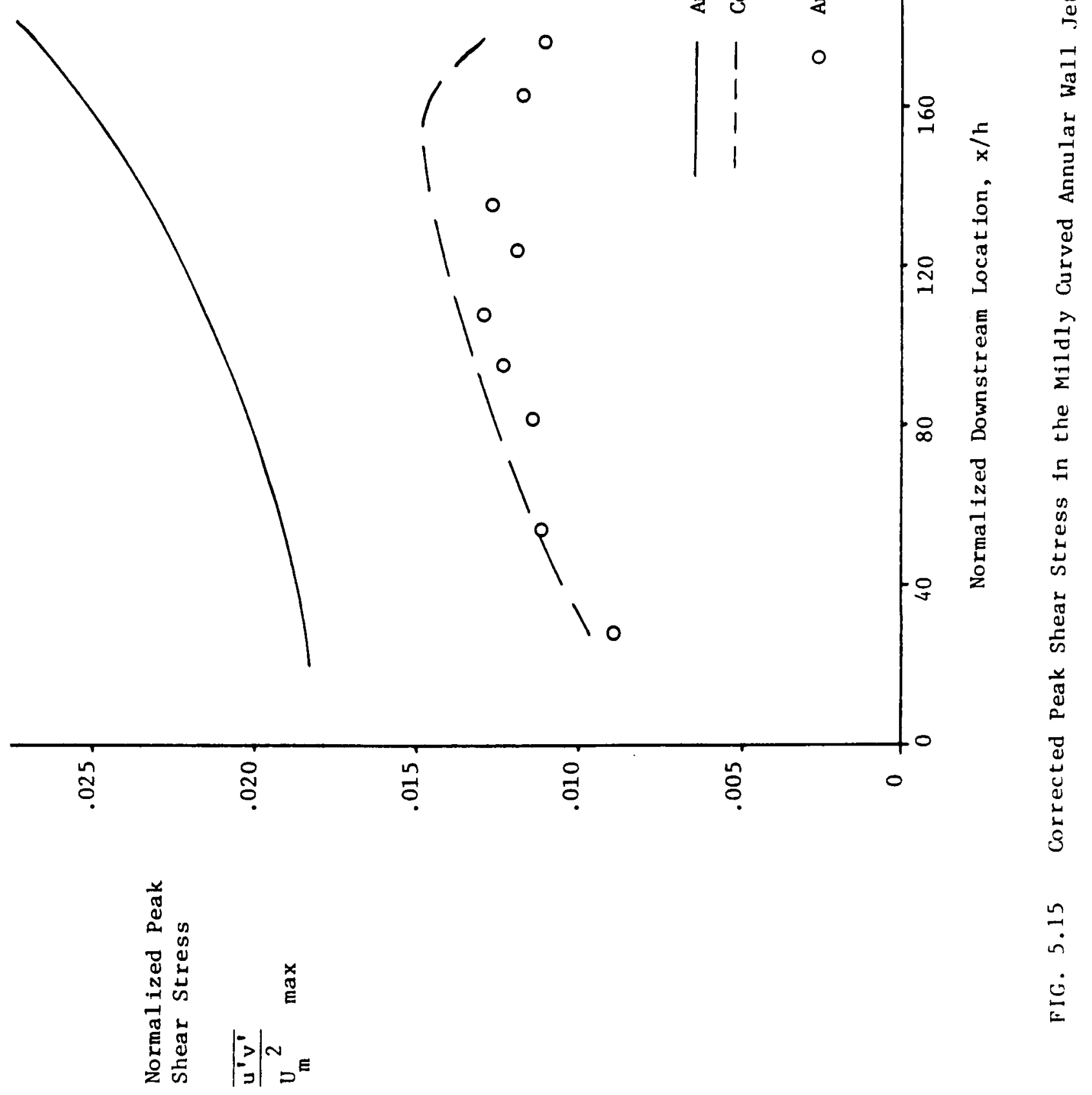




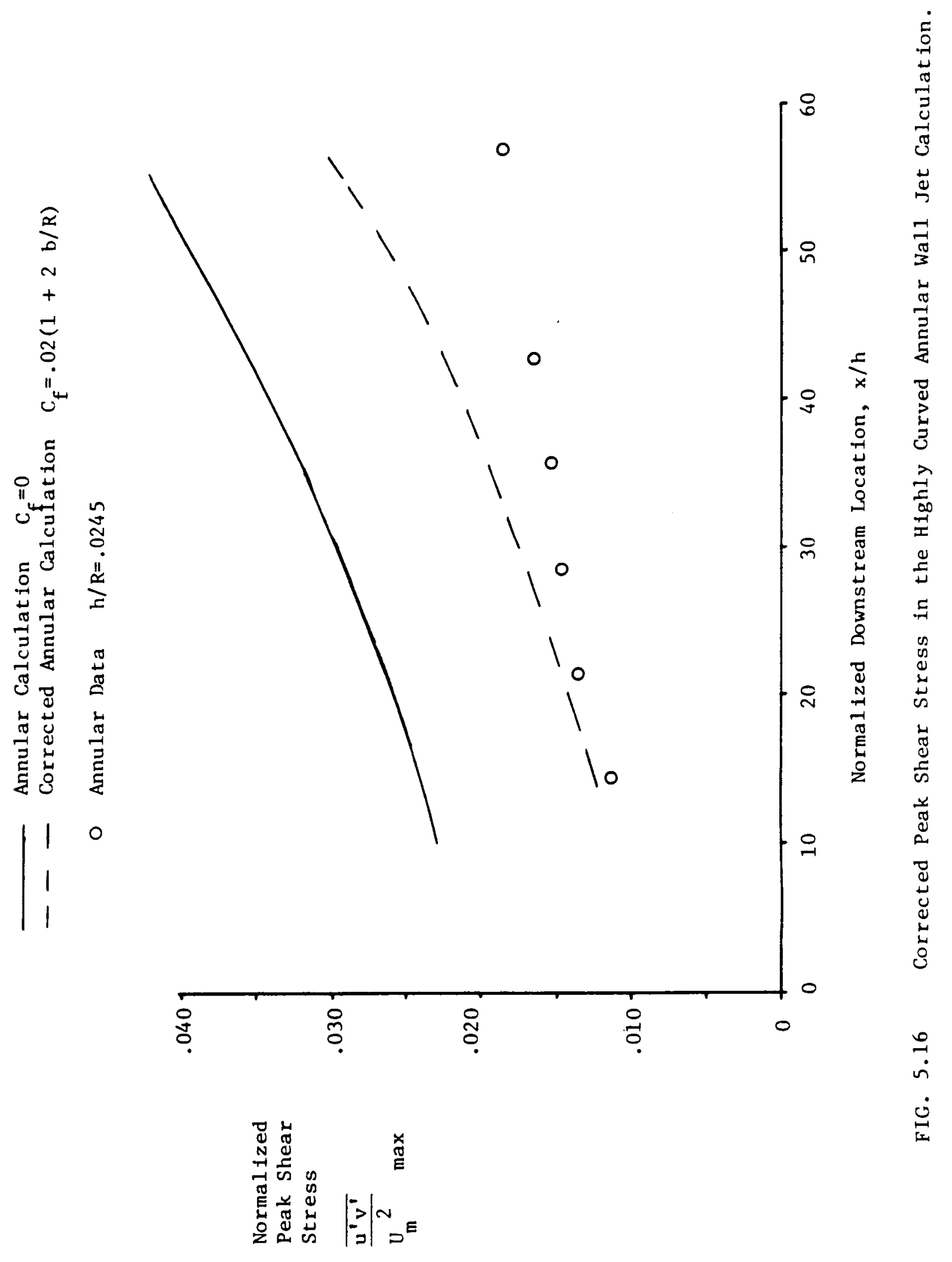




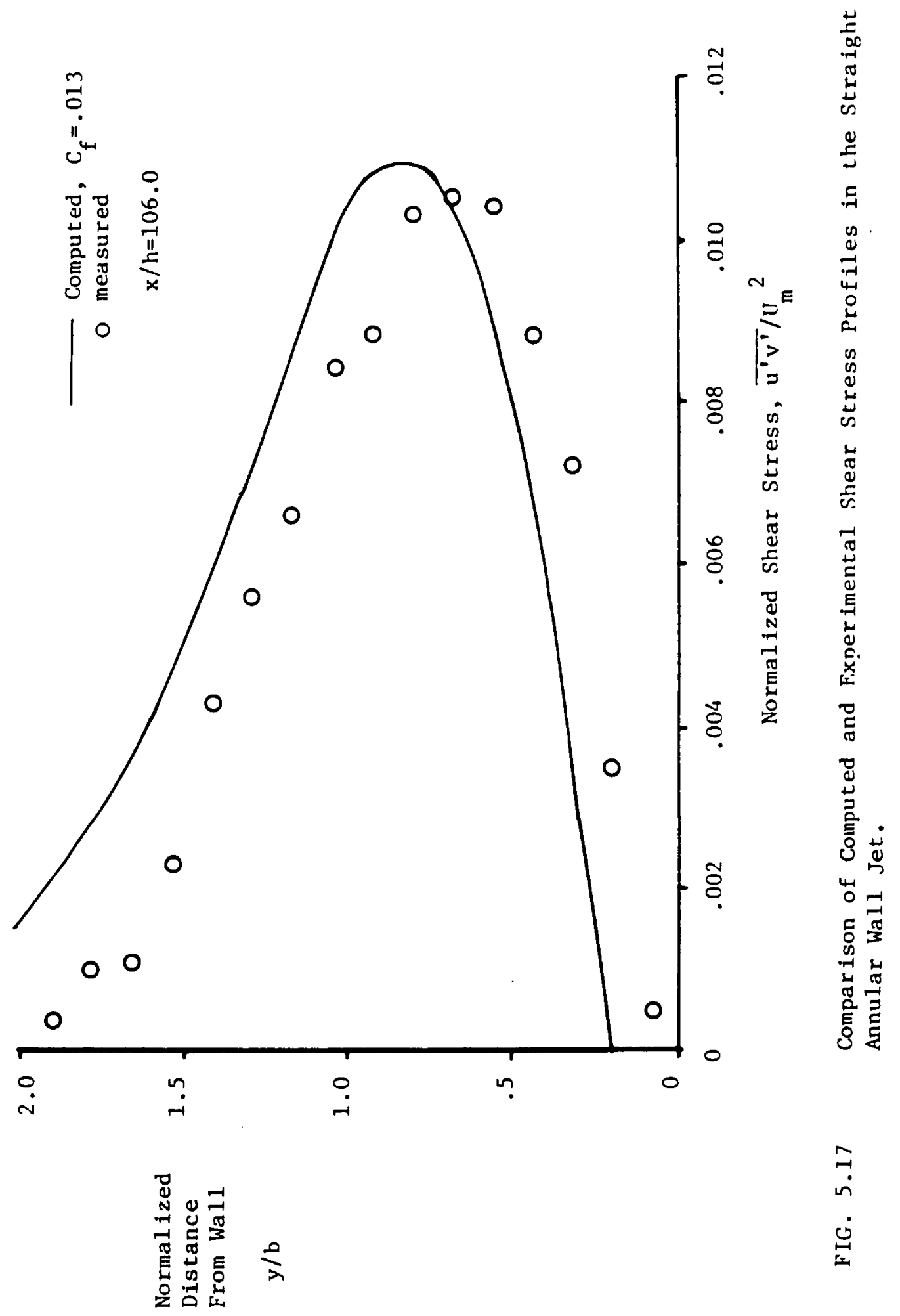




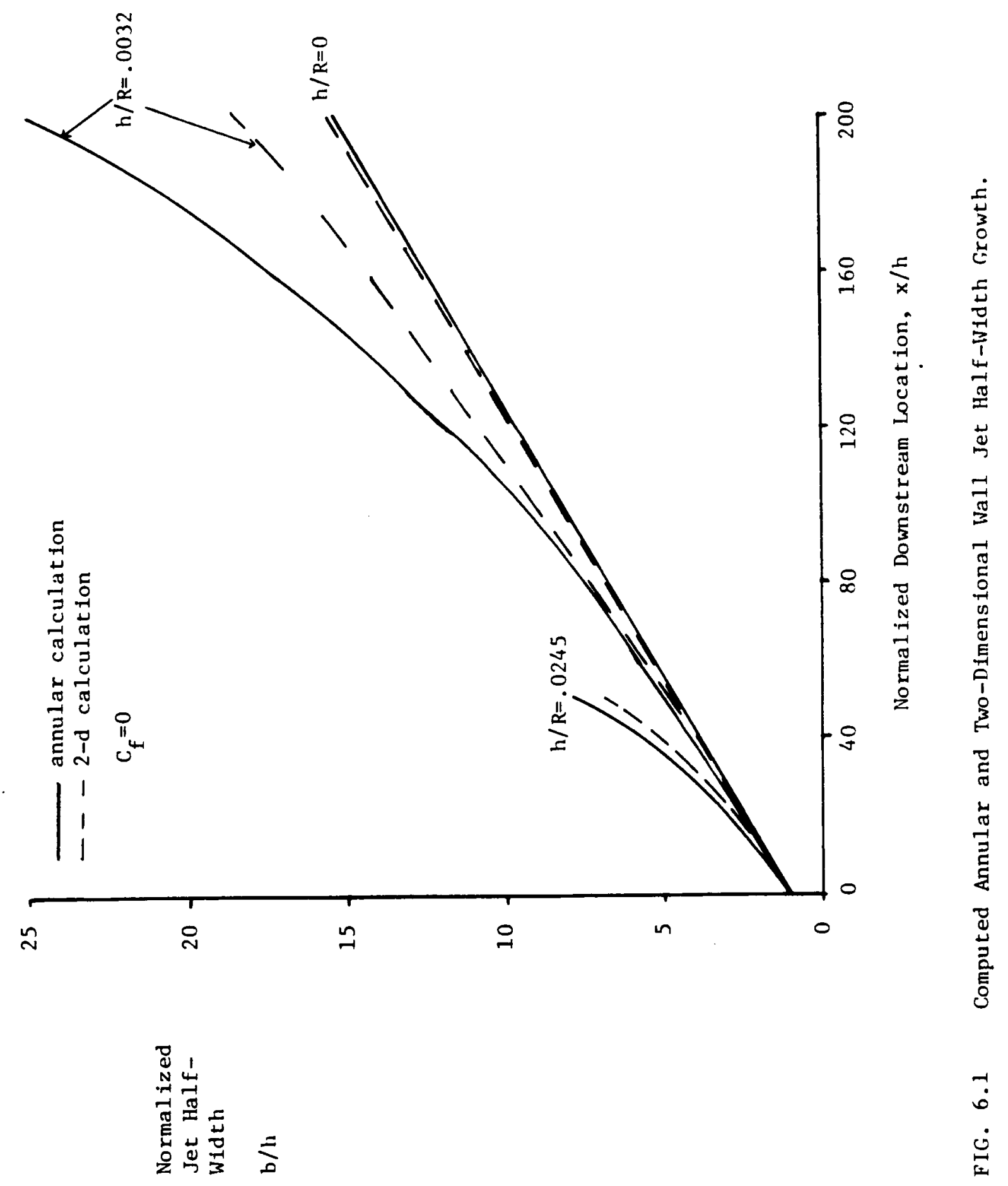




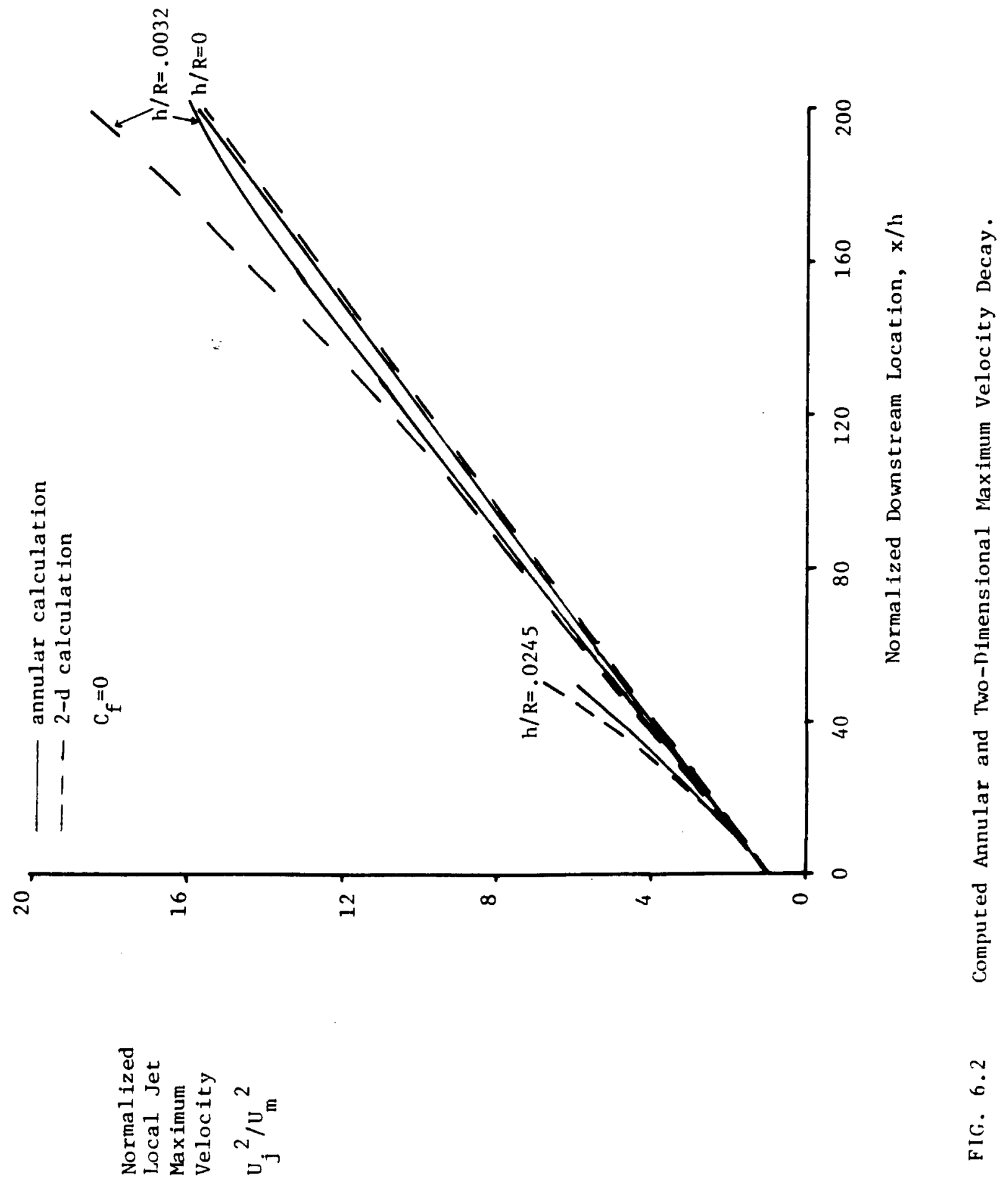




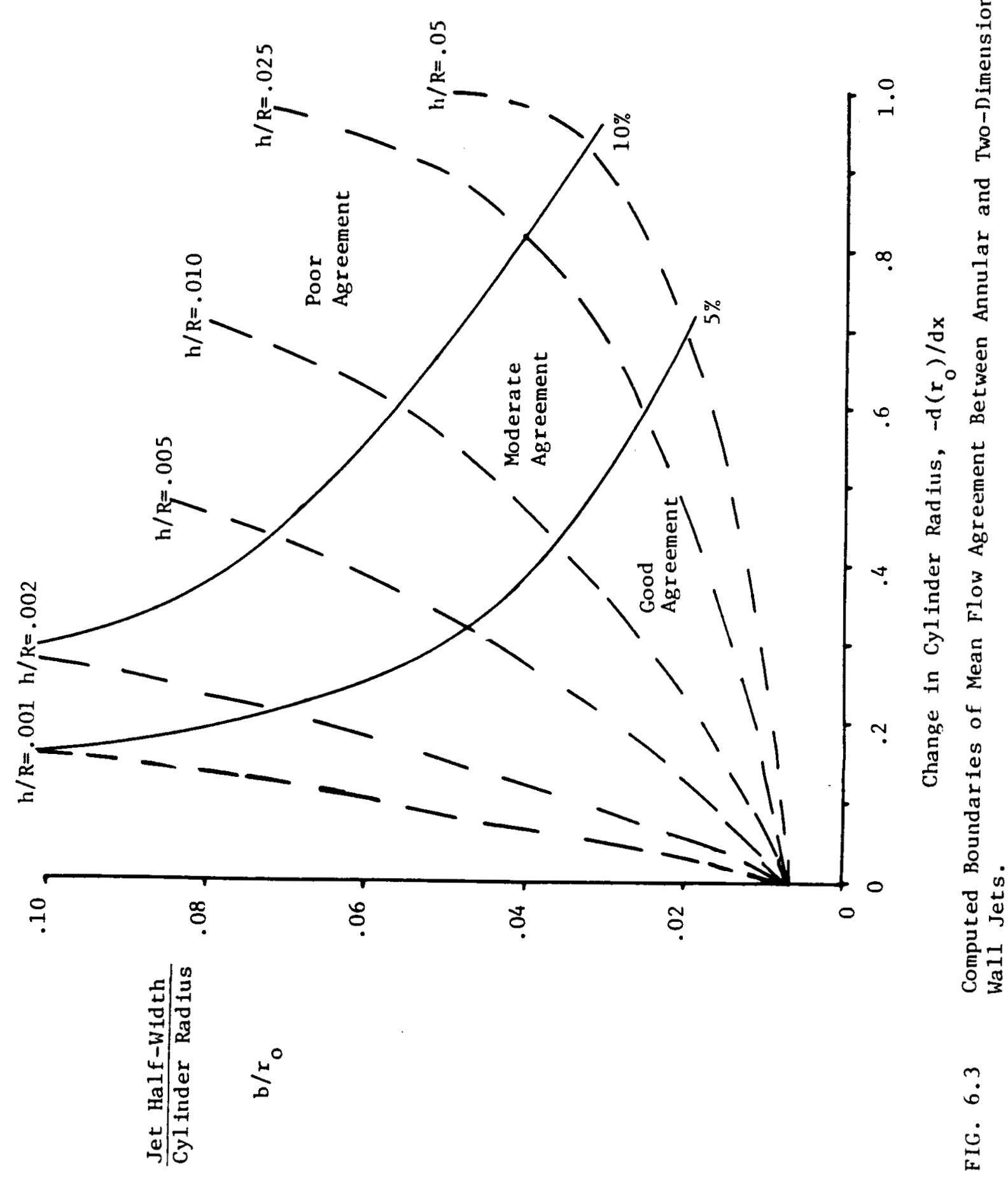


$m$
0
10
$u^{4+1}$

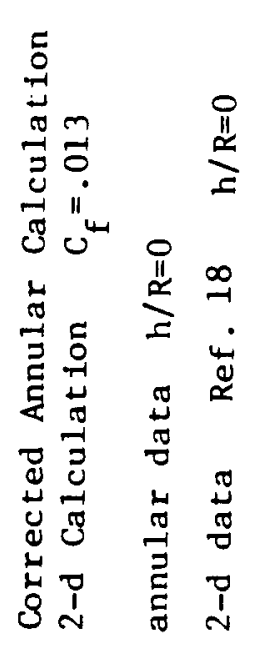

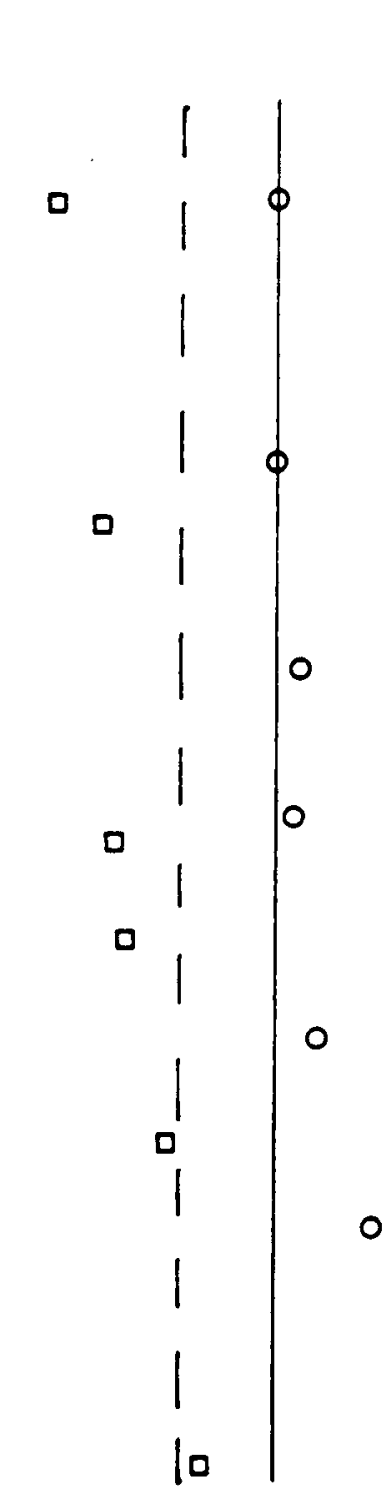

$\mid \begin{array}{lll}1 & 0 & 0 \\ 1 & & \end{array}$

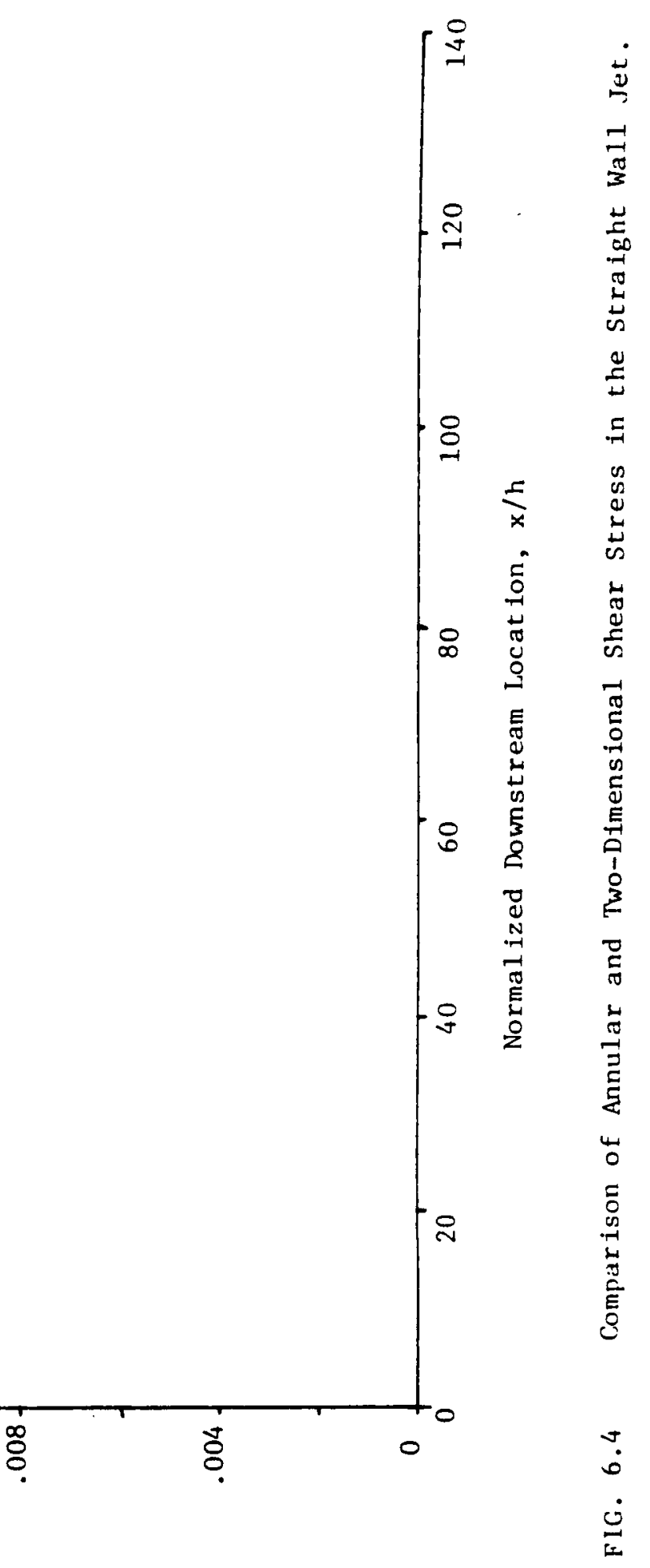

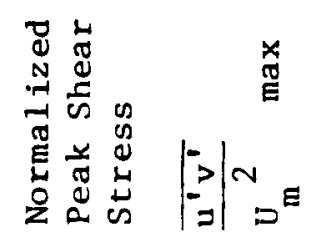




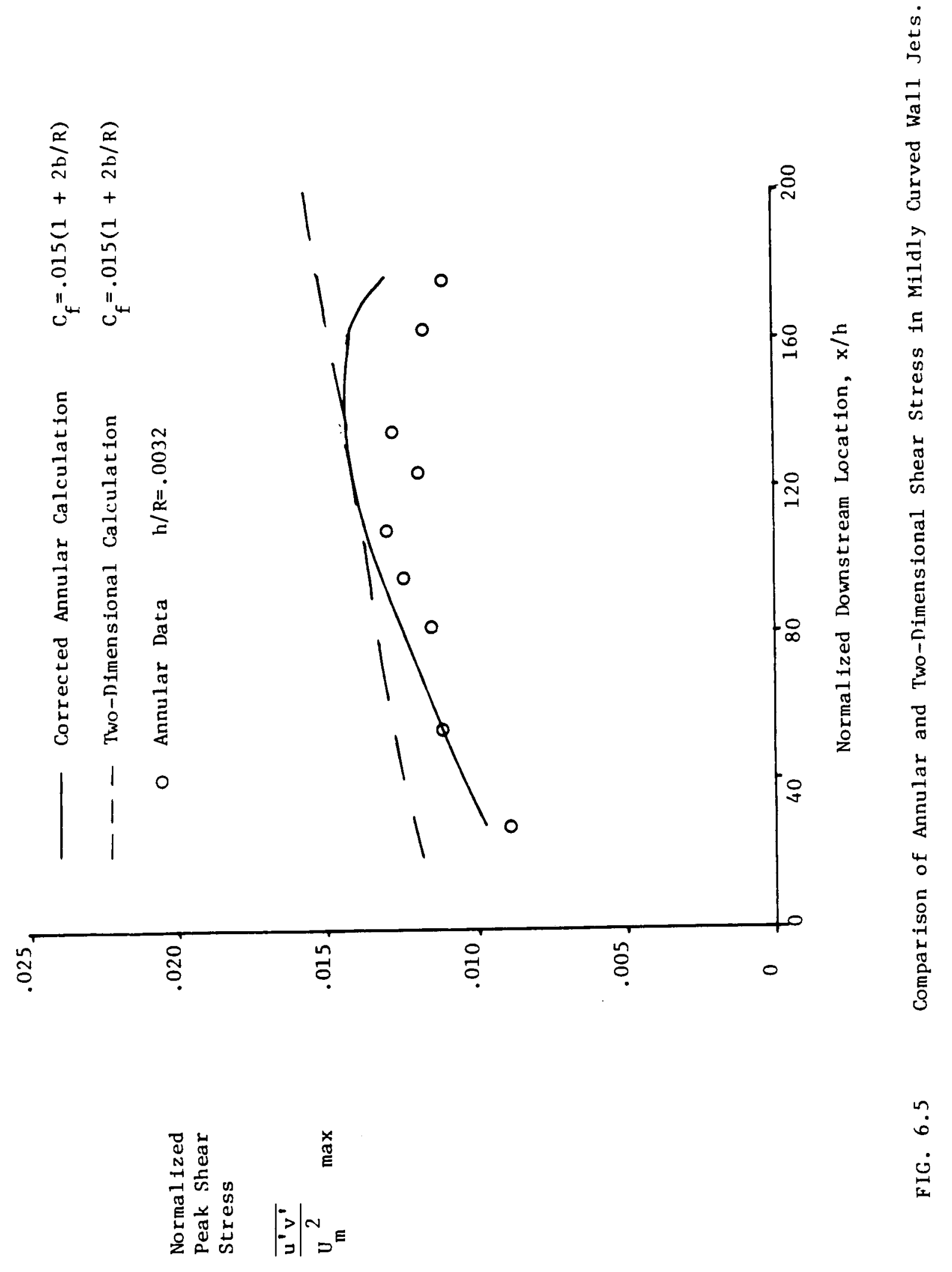




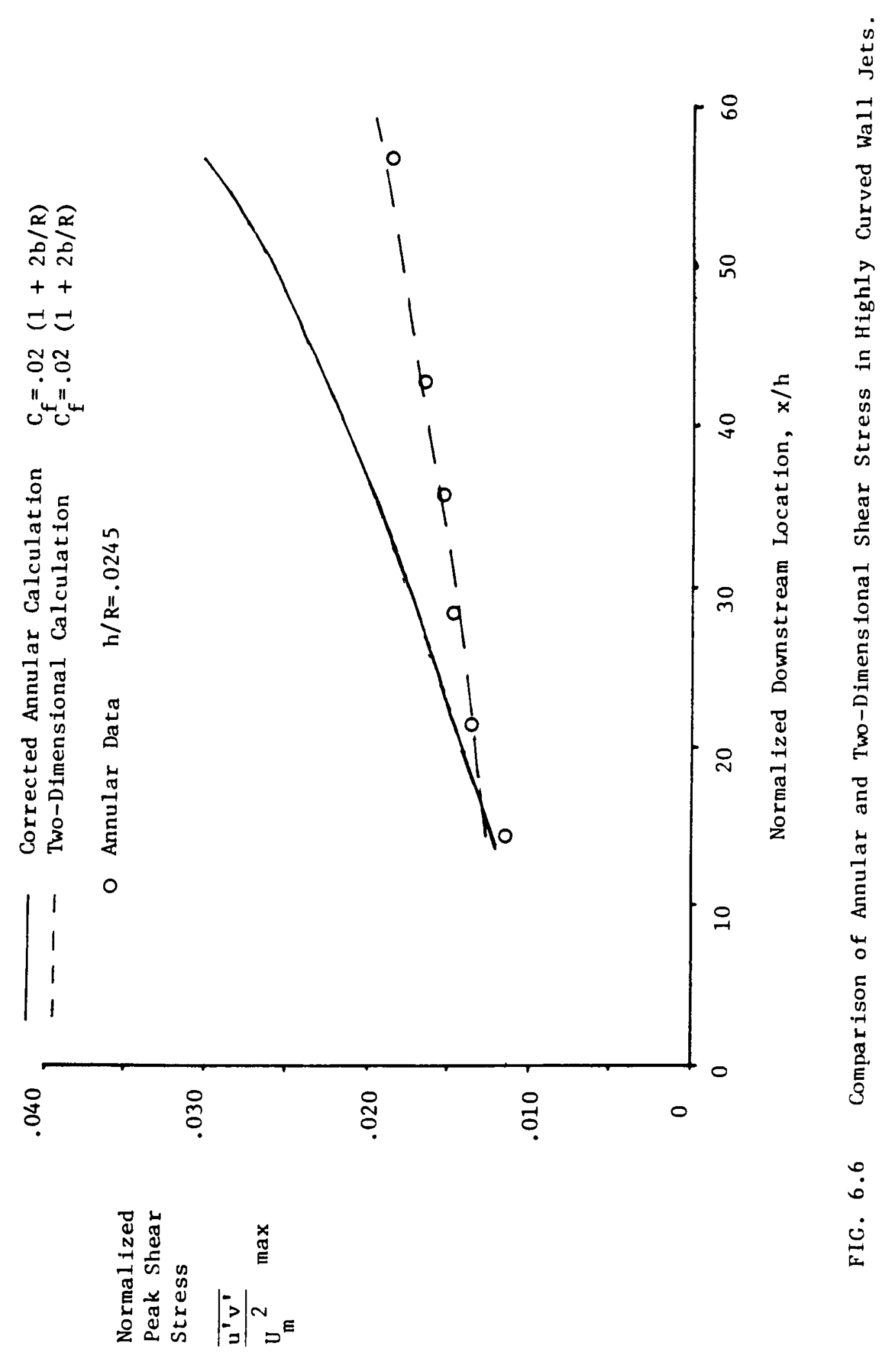


- $h / R=.0595 \quad \operatorname{Ref.} 18$

- $h / R=.0032 \quad \operatorname{Ref} .20 \quad R e=1.1 \times 10_{4}^{4}$

- $h / R=.0032$ Ref. $20 \quad R e=4.0 \times 10^{4}$

Annular Data:
$\Delta \mathrm{h} / \mathrm{R}=.0245$
D $h / R=.0032$
○ $h / R=0$
$\mathrm{Be}=0.9 \times 10_{4}^{4}$
$\operatorname{Re}=0.9 \times 10_{4}^{4}$
$\operatorname{Re}=0.9 \times 10^{4}$

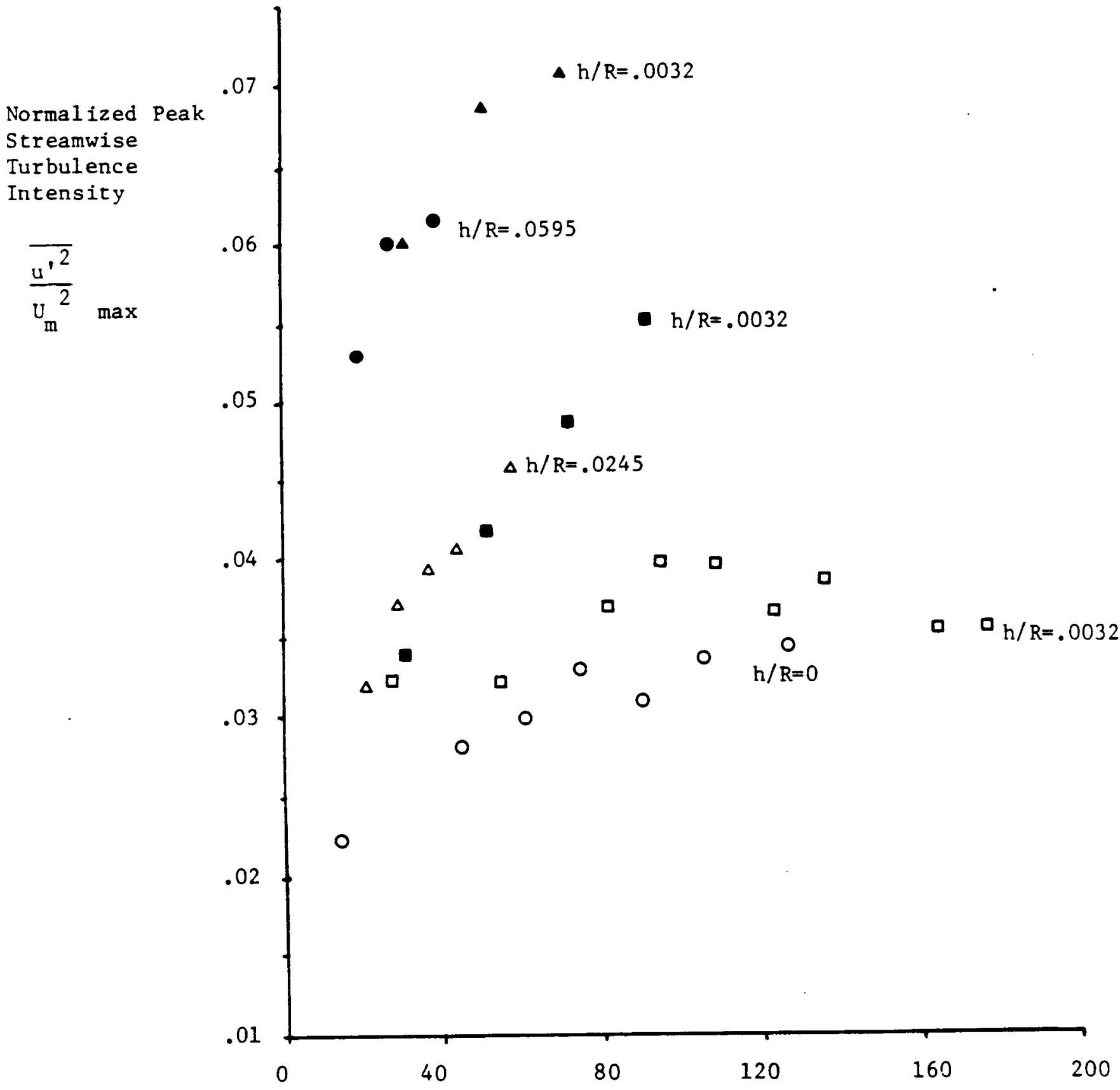

Normalized Downstream Location, $x / h$

FIG. 6.7 Streamwise Curvature Effects on Peak Streamwise Normal Stress. 


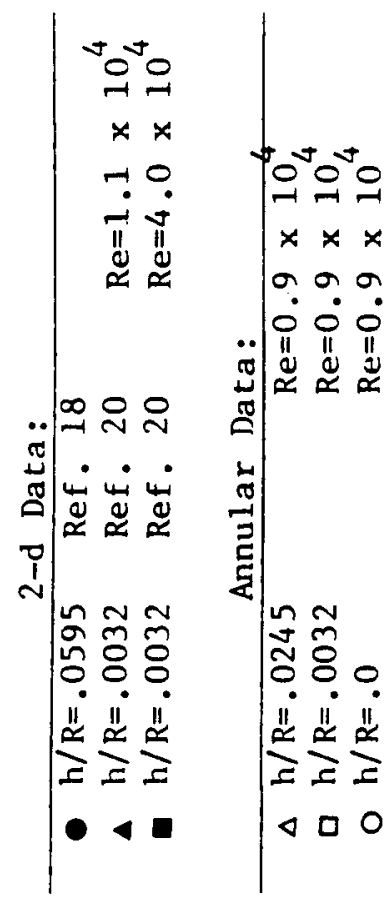

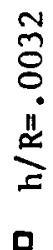

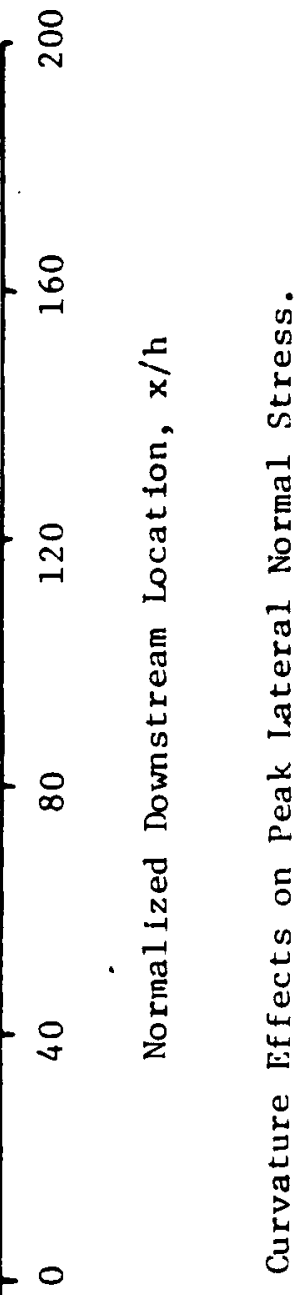

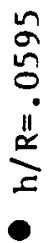
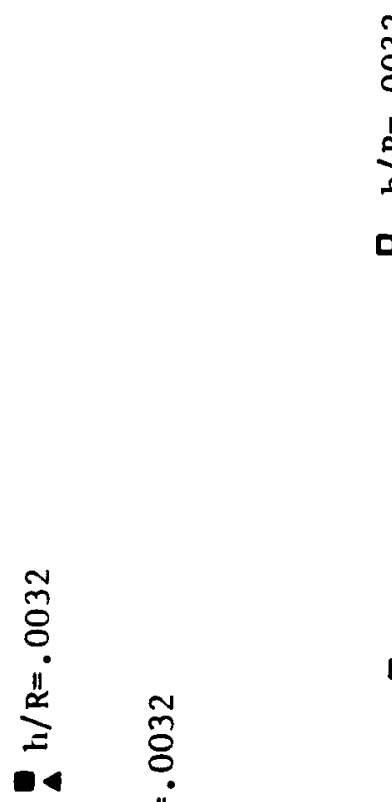

ס

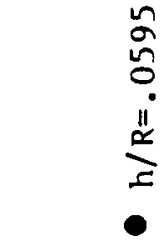

กิ

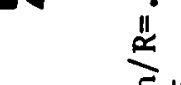

는
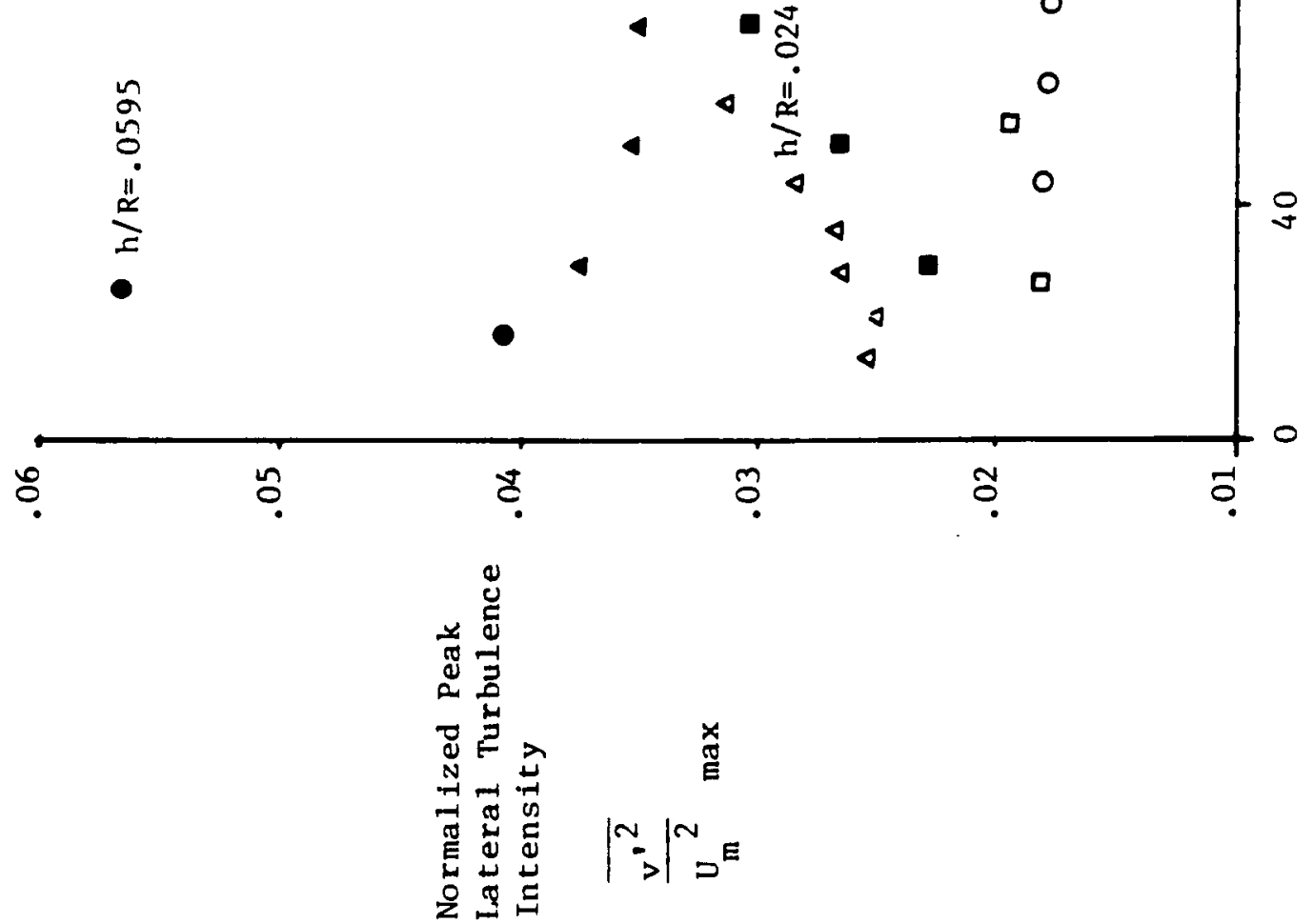

$\infty$
$\dot{0}$
$\dot{0}$ 

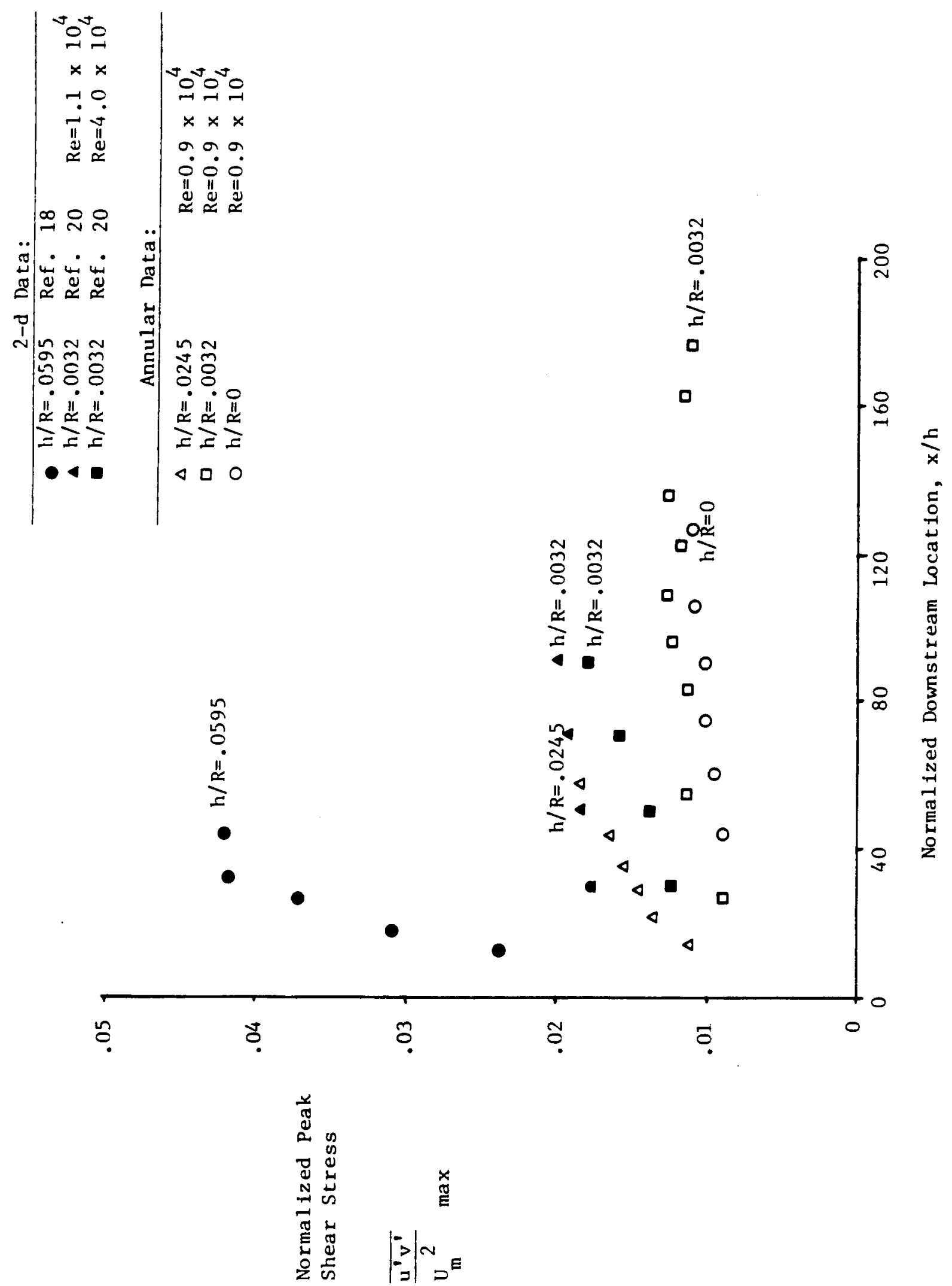

告

o

E 


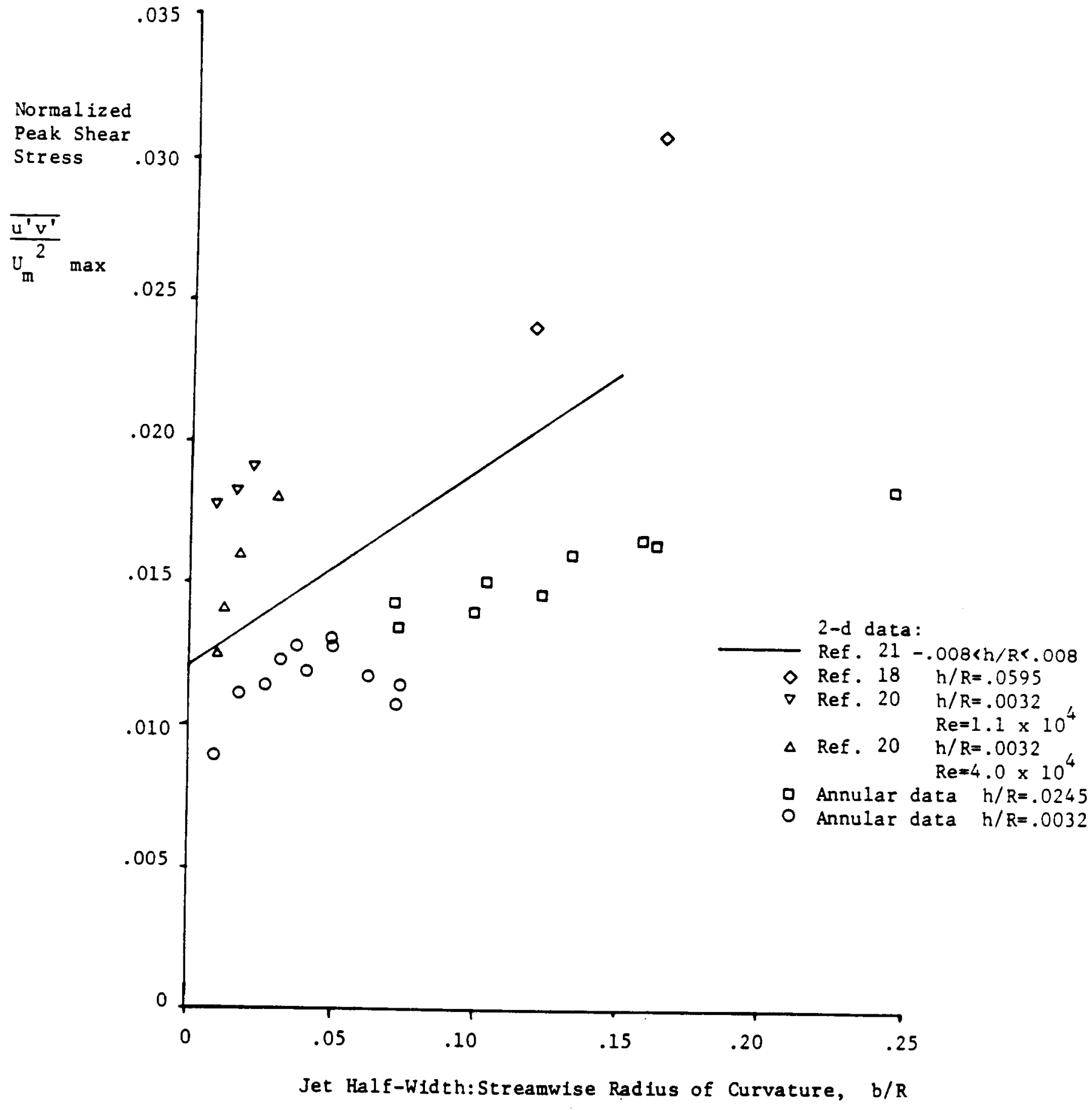

FIG. 6.10 Correlation of Peak Shear Stress with Streamwise Curvature. 